\title{
Reconstruction, Identification and Implementation Methods for Spiking Neural Circuits
}

A thesis submitted to the University of Sheffield for the degree of Doctor of Philosophy

\section{Dorian Florescu}

Department of Automatic Control and Systems Engineering 

To my family 



\section{Acknowledgements}

I'd like to thank by supervisors Daniel Coca and Stephen Billings for being by my side during my years as a $\mathrm{PhD}$ student. Moreover, I would like to thank my father for the very useful discussions that inspired me to overcome some of the theoretical barriers I encountered in my research. Many thanks go to Sarah Bell, who gave me a lot of useful writing up time management tips.

I thank all my colleagues from the Automatic Control and Systems Engineering department for their friendship and support, in particular to Adrian Alecu, Krishnanathan Kirubhakaran, Andrew Hills and Veronica Biga for the funny moments shared and the advices received at times of hardship.

Many thanks go to my Sheffield friends Ines, Julia, Nikki, Irina, Madalina, Mihai for the nice moments spent together and the good laughs that always improved my mood.

And last, but not least, a massive thanks to my mother and sister for their compassion during my hard times and enthusiasm during my successes. 


\section{Abstract}

Integrate-and-fire (IF) neurons are time encoding machines (TEMs) that convert the amplitude of an analog signal into a non-uniform, strictly increasing sequence of spike times.

This thesis addresses three major issues in the field of computational neuroscience as well as neuromorphic engineering.

The first problem is concerned with the formulation of the encoding performed by an IF neuron. The encoding mechanism is described mathematically by the $t$-transform equation, whose standard formulation is given by the projection of the stimulus onto a set of inputdependent frame functions. As a consequence, the standard methods reconstruct the input of an IF neuron in a space spanned by a set of functions that depend on the stimulus. The process becomes computationally demanding when performing reconstruction from long sequences of spike times.

The issue is addressed in this work by developing a new framework in which the IF encoding process is formulated as a problem of uniform sampling on a set of input independent time points. Based on this formulation, new algorithms are introduced for reconstructing the input of an IF neuron belonging to bandlimited as well as shift-invariant spaces. The algorithms are significantly faster, whilst providing a similar level of accuracy, compared to the standard reconstruction methods.

Another important issue calls for inferring mathematical models for sensory processing systems directly from input-output observations. This problem was addressed before by performing identification of sensory circuits consisting of linear filters in series with ideal IF neurons, by reformulating the identification problem as one of stimulus reconstruction. The result was extended to circuits in which the ideal IF neuron was replaced by more biophysically realistic models, under the additional assumptions that the spiking neuron parameters are known a priori, or that input-output measurements of the spiking neuron are available.

This thesis develops two new identification methodologies for [Nonlinear Filter]-[Ideal IF] and [Linear Filter]-[Leaky IF] circuits consisting of two steps: the estimation of the spiking neuron parameters and the identification of the filter. The methodologies are based on the reformulation of the circuit as a scaled filter in series with a modified spiking neuron.

The first methodology identifies an unknown [Nonlinear Filter]-[Ideal IF] circuit from input-output data. The scaled nonlinear filter is estimated using the NARMAX identification methodology for the reconstructed filter output.

The [Linear Filter]-[Leaky IF] circuit is identified with the second proposed methodology by first estimating the leaky IF parameters with arbitrary precision using specific stimuli sequences. The filter is subsequently identified using the NARMAX identification methodology. 
The third problem addressed in this work is given by the need of developing neuromorphic engineering circuits that perform mathematical computations in the spike domain.

In this respect, this thesis developed a new representation between the time encoded input and output of a linear filter, where the TEM is represented by an ideal IF neuron. A new practical algorithm is developed based on this representation. The proposed algorithm is significantly faster than the alternative approach, which involves reconstructing the input, simulating the linear filter, and subsequently encoding the resulting output into a spike train. 


\section{Contents}

Nomenclature $\quad x$

$\begin{array}{lll}\text { Acronyms } & \text { xii }\end{array}$

1 Introduction 1

1.1 Background . . . . . . . . . . . . . . . . . . . 1

1.2 Motivation . . . . . . . . . . . . . . . . 2

1.3 Overview of the thesis $\ldots \ldots \ldots \ldots \ldots$

2 Time encoding and decoding in bandlimited and shift-invariant spaces 6

2.1 Nonuniform sampling and reconstruction of bandlimited

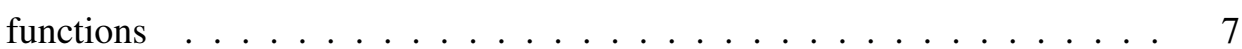

2.2 Time encoding and decoding in bandlimited spaces . . . . . . . . . . 10

2.2 .1 The ideal IF neuron . . . . . . . . . . . . . . . . . . . . . 11

2.2.2 The ideal IF neuron with refractory period . . . . . . . . . . . . 14

2.2 .3 The leaky IF neuron . . . . . . . . . . . . . . . 15

2.2.4 The leaky IF neuron with random threshold . . . . . . . . . . . . 16

2.2.5 The Hodgkin-Huxley neuron . . . . . . . . . . . . . . . . 18

2.2.6 The asynchronous sigma-delta modulator . . . . . . . . . . . . . 19

2.3 Time encoding and decoding in shift-invariant spaces . . . . . . . . . 22

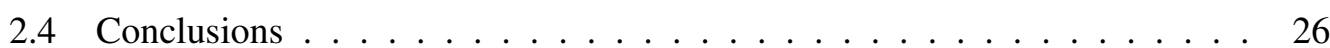

3 A novel framework for reconstructing bandlimited signals encoded by integrate$\begin{array}{lr}\text { and-fire neurons } & 28\end{array}$

3.1 A new method of reconstructing functions from local averages . . . . . . . 29

3.2 Direct reconstruction algorithms for inputs encoded with ideal IF neurons . 34

3.3 The integrate-and-fire neuron as a uniform sampler . . . . . . . . . . 35

3.4 Fast indirect reconstruction algorithms for inputs encoded with ideal IF neu-

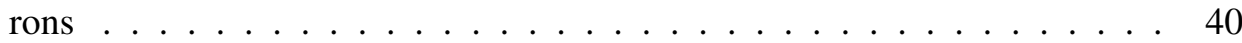

3.5 Numerical study . . . . . . . . . . . . . . . . . . . . . . . . . 43 
3.5.1 Numerical study for Algorithm 3.1 . . . . . . . . . . . . . . 45

3.5.2 Numerical study for Algorithm 3.2 . . . . . . . . . . . . . . 49

3.5.3 Error evaluation for the interpolation step of the proposed algorithms 51

3.6 Conclusions ......................... 53

4 A novel reconstruction framework in shift-invariant spaces for signals encoded with integrate-and-fire neurons $\quad 55$

4.1 A new non-iterative method for reconstructing signals in shift-invariant spaces from spike trains generated with IF-TEMs _ . . . . . . . . . . 57

4.2 Direct reconstruction algorithms for inputs encoded with ideal IF neurons . 64

4.3 Fast indirect reconstruction algorithms for inputs encoded with ideal IF neu-

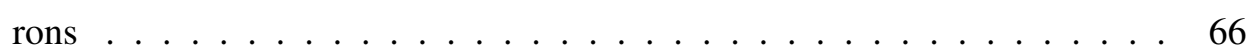

4.4 Numerical study . . . . . . . . . . . . . . . . . . . . . 72

4.4.1 Comparative numerical study of the iterative algorithms . . . . 73

4.4.2 Comparative numerical study of the non-iterative algorithms . . . . 74

4.5 Conclusions . . . . . . . . . . . . . . . . . 75

5 A new approach to the identification of sensory processing circuits based on $\begin{array}{ll}\text { spiking neuron data } & \mathbf{7 7}\end{array}$

5.1 Identification of spiking neural circuits . . . . . . . . . . . 78

5.1.1 Identification of [Linear Filter]-[Ideal IF] circuits . . . . . . . . . 79

5.1.2 Identification methods for different circuit structures . . . . . . . 82

5.2 The NARMAX identification methodology . . . . . . . . . . . . . . 85

5.2.1 An overview of the NARMAX model . . . . . . . . . . . 85

5.2.2 The orthogonal least squares estimator . . . . . . . . . . . 86

5.2.3 The orthogonal forward regression algorithm . . . . . . . . . 88

5.2.4 The generalised frequency response functions . . . . . . . . . . . 90

5.3 A new method for the identification of [Nonlinear Filter]-[Ideal IF] circuits 93

5.3.1 Problem statement . . . . . . . . . . . . . . 93

5.3 .2 Numerical study . . . . . . . . . . . . . . . . . 95

5.4 A new methodology for the identification of [Linear Filter]-[Leaky IF] circuits 98

5.4.1 Problem statement . . . . . . . . . . . . . . . 98

5.4 .2 Numerical study . . . . . . . . . . . . . . . . . . . . . . 102

5.5 Conclusions . . . . . . . . . . . . . . . . . . . . . . 105

6 A new method for implementing linear filters in the spike domain 106

6.1 Problem statement . . . . . . . . . . . . . . . . . . . 107

6.2 Direct computation of spike times . . . . . . . . . . . . . 109

6.3 Numerical study . . . . . . . . . . . . . . . . . . . . . . . . 112 
Contents

6.4 Conclusions . . . . . . . . . . . . . . . . . . . . . . 116

7 Conclusions and future work 118

Bibliography 


\section{List of Figures}

2.1 The ideal IF neuron. . . . . . . . . . . . . . . . . . . . 11

2.2 Single-input multi-output population of $N$ ideal IF neurons. . . . . . . . . . 13

2.3 The ASDM circuit. . . . . . . . . . . . . . . . . . . . . . . 19

3.1 The relationship between functions $U$ and $\psi$ for a bandlimited input function. 37

3.2 Input functions $\left\{u^{j}(t)\right\}_{i=1,2,3} \ldots \ldots \ldots \ldots \ldots$

3.3 Reconstruction SER of Algorithm 3.1 for different values of $\bar{\Omega}_{M}$. . . . . . 46

3.4 Average SER of 100 inputs reconstructed with Algorithm 3.1 and the standard algorithm for an increasing number of iterations. . . . . . . . . . . 46

3.5 Normalised reconstruction errors $E^{1}(t)$ of Algorithm 3.1 and the standard algorithm corresponding to A) $n=1$ and B) $n=10^{4}$ iterations. . . . . . 47

3.6 Normalised residual error of Algorithm 3.1 and the standard algorithm for an increasing number of iterations. . . . . . . . . . . . . . . 48

3.7 Comparison between Algorithm 3.1 and Lazar algorithm for 100 input sequences. Probability density functions for $10^{3}$ iterations corresponding to A1) reconstruction time and A2) reconstruction accuracy, and for $10^{4}$ iterations corresponding to $\mathrm{B} 1$ ) reconstruction time and $\mathrm{B} 2$ ) reconstruction accuracy . . . . . . . . . . . . . . . . .

3.8 Computation time as a function of the number of spikes used in reconstruction for $n$ iterations: A) Algorithm 3.1 and B) the standard algorithm. . . . 50

3.9 Reconstruction SER of Algorithm 3.2 for different values of $\bar{\Omega}_{M}$. . . . . . 50

3.10 Comparison between Algorithm 3.2 and the Lazar-Pnevmatikakis algorithm. Probability density functions corresponding to A) reconstruction time and B) reconstruction accuracy, estimated based on 100 input sequences. . . . . 51

3.11 The normalised errors corresponding to input functions $\left\{u^{1}, u^{2}, u^{3}\right\}$ for A) Algorithm 3.2 and B) the Lazar-Pnevmatikakis algorithm. . . . . . . . . 52

3.12 Computation time as function of the number of spikes used in reconstruction. 53

3.13 The average normalised error $e(t)$, corresponding to the linear interpolation of inputs $\left\{u^{j}(t)\right\}_{j=1, \ldots, 100} \ldots \ldots \ldots \ldots \ldots \ldots$ 
4.1 Input functions $\left\{u^{j}(t)\right\}_{j=1,2,3} \ldots \ldots \ldots \ldots \ldots$

4.2 Average computation time (top) and SER (bottom) for 100 inputs reconstructed with algorithms 4.1 and $4.3 \ldots \ldots \ldots \ldots$. . . . . . . . . 74

4.3 Computation time as a function of the number of spikes used in reconstruction for $n$ iterations: A) Algorithm 4.3 and B) Algorithm 4.1 . . . . . . . . 75

4.4 Comparison between Algorithm 4.2 and Algorithm 4.4. Probability density functions corresponding to: A) reconstruction time and $\mathrm{B}$ ) reconstruction

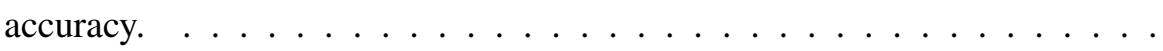

4.5 Comparison between Algorithm 4.2 and Algorithm 4.4. Probability density functions corresponding to: A) reconstruction time and B) reconstruction

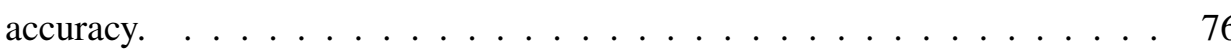

5.1 Linear filter in cascade with an IF neuron, modelling the dendritic tree and the spiking neuron, respectively. . . . . . . . . . . . . . . . 79

5.2 Input-output equivalent model for the [Filter]-[Ideal IF] circuit. . . . . . . . 80

5.3 The estimation of $h_{b}$ reformulated as a reconstruction problem. . . . . . . 81

5.4 MISO [Filter]-[Ideal IF] circuit with multisensory integration. . . . . . . . 82

5.5 Volterra expansion of the filter in a [Nonlinear Filter]-[Ideal IF] circuit. . . . 84

5.6 The structure of the circuit proposed for identification. . . . . . . . . . . . 93

5.7 Original $\left(a_{1}\right)$ and equivalent $\left(a_{2}\right)$ neural circuit. . . . . . . . . . . 95

5.8 The generalised frequency response functions associated with system (5.13). 96

5.9 Input functions $u_{t r}, u_{\text {val }}$ and the corresponding output responses $v_{t r}, v_{\text {val }} . \quad$. 97

5.10 Function $v_{v a l}^{*}$ (up) and validation error $E$ (down) . . . . . . . . . . . . 98

5.11 The error functions between the original GFRFs $H_{1}, H_{2}$ and the ones computed from the NARX model scaled by $b$, namely $b H_{1}^{*}, b H_{2}^{*} \ldots \ldots$. . . . . 99

5.12 Original $\left(b_{1}\right)$ and equivalent $\left(b_{2}\right)$ neural circuit. . . . . . . . . . 100

5.13 The output of circuit $\left(b_{2}\right)$ for inputs $u_{0}, u_{1}$ and $u_{2} \ldots \ldots$. . . . . . 103

5.14 Function $\bar{f}(x)$ and the real value of parameter RC . . . . . . . . . . . 104

5.15 Magnitude plot of system (5.27). . . . . . . . . . . . . . . . . . . . . . 104

5.16 The error between the original and the identified magnitude curve scaled by $b .105$

6.1 Linear filter implementation in the spike domain. . . . . . . . . . . . . . 108

6.2 Impulse response of filter $G$. . . . . . . . . . . . . . . . . . . . 113

6.3 Input and output of filter G. . . . . . . . . . . . . . . . . . . 114

6.4 Normalized errors $E_{1}$ and $E_{2}$ for the direct and indirect methods, respectively. 115

6.5 Input and output of filter G. . . . . . . . . . . . . . . . . . 116 


\section{List of Tables}

6.1 Computing Times and MSEs of the Direct and Indirect Method for a Bandlimited Input . . . . . . . . . . . . . . . . . . . . 114

6.2 Computing Times and MSEs for the Direct and Indirect Method for a Non-

Bandlimited Input . . . . . . . . . . . . . . . . 116 


\title{
Nomenclature
}

\author{
A list of the variables and notation used in this thesis is defined below. The definitions \\ and conventions set here will be observed throughout unless otherwise stated. For a list of \\ acronyms, please consult page xii. \\ $1_{[0,1]} \quad$ characteristic function \\ $[0,1] \quad$ closed interval \\ Id identity matrix \\ $\circ \quad$ function composition \\ $\mathcal{F}^{-1} \quad$ inverse Fourier transform \\ $\mathcal{H}_{\Omega}^{M} \quad$ space of trigonometric polynomials with $2 M+1$ dimensions \\ $\mathcal{P}_{H} \quad$ orthogonal projection onto space $H$ \\ $\|\cdot\|_{H} \quad$ norm in space $H$ \\ $\langle\cdot\rangle_{H} \quad$ inner product in space $H$ \\ ln natural logarithm \\ sgn sign function \\ $\operatorname{supp}(f) \quad$ support function \\ $\varepsilon \quad$ simulation sampling time \\ $\widehat{f} \quad$ Fourier transform of function $f$ \\ ] $0,1[\quad$ open interval \\ a.e. almost everywhere \\ $i \quad$ unit imaginary number
}




$\begin{array}{ll}G^{+} & \text {Moore-Penrose pseudoinverse of matrix } G \\ G^{T} & \text { transpose of matrix } G \\ \delta_{a b} & \text { Kronecker delta function } \\ \hookrightarrow & \text { embedding between two spaces } \\ \mathbb{C} & \text { set of complex numbers } \\ \mathbb{R} & \text { set of real numbers } \\ \mathcal{C}^{*} & \text { adjoint of operator } \mathcal{C} \\ \mathcal{I} & \text { identity operator } \\ P W_{\Omega} & \text { Paley-Wiener space of bandwidth } \Omega \\ \mathbb{R}_{+} & \text {set of positive real numbers } \\ \text { card } & \text { cardinal function } \\ \text { inf } & \text { infimum function } \\ \mathcal{C}^{*} & \text { conjugate of a complex number }\end{array}$




\section{Acronyms}

A/D analog-to-digital.

ARMAX AutoRegressive Moving Average with eXogenous inputs.

ARX AutoRegressive with eXogenous inputs.

ASDM asynchronous sigma-delta modulator.

BIBO bounded-input bounded-output.

C-TEM crossing TEM.

CIM channel identification machine.

DSP dendritic stimulus processor.

ERR error reduction ratio.

ESR error-to-signal-ratio.

GFRF generalised frequency response function.

IF integrate-and-fire.

IF-TEM integrate-and-fire TEM.

LIF leaky IF.

mCIM multisensory CIM.

MISO multiple-input single-output.

MPO model predicted output.

NARMAX Nonlinear AutoRegressive Moving Average with eXogenous inputs. 
NARX Nonlinear AutoRegressive with eXogenous inputs.

NIM neuron identification machine.

OFR orthogonal forward regression.

OLS orthogonal least squares.

OSA One-Step-Ahead.

PRC phase response curve.

SIMO single-input multiple-output.

SIS shift-invariant space.

TDM time decoding machine.

TEM time encoding machine. 



\section{Chapter 1}

\section{Introduction}

\subsection{Background}

A fundamental problem that remains unsolved in neuroscience is determining how the information from the outside world is represented by the brain. The overall functioning of the brain is based on elementary computational units called neurons, which communicate with each other by transmitting and receiving signals through their axons and dendrites, respectively (Churchland and Sejnowski, 1992).

The neurons respond to analog inputs with action potentials, or spikes, which are generated when the neuron membrane potential exceeds a threshold value (Izhikevich, 2007). There is an ongoing debate among neuroscientists on whether the information is represented in the spike train as a temporal code, for which individual spike times encode independently of each other, or a rate code, where the information is contained in the correlation between different spikes (Dayan and Abbott, 2001).

The integrate-and-fire (IF) neuron, introduced by Lapicque (1907), is one of the most common models of the spiking neuron based on the temporal code (Tuckwell, 1988). The IF model belongs to the larger class of time encoding machines (TEMs), defined as realtime asynchronous mechanisms that encode the amplitude information of a function into a time sequence (Lazar and Tóth, 2003).

TEMs are an important tool in information processing as they represent sampling devices that, in contrast to classical analog-to-digital (A/D) converters, are not affected by resolution reduction when implemented on low power circuits (Roza, 1997).

The encoding operation performed by a TEM is described mathematically by an equation called the $t$-transform, introduced by Lazar and Tóth (2003). This operation can be interpreted as the projection of the stimulus $u$ onto a set of functions that depend on the output spike times, and thus on $u$ (Lazar, 2004). Based on this formulation, several algorithms have been proposed for reconstructing a bandlimited input $u$ from the output $\mathcal{T}_{u}=\left\{t_{k}\right\}_{k \in \mathbb{Z}}$ 
generated by an IF neuron (Lazar, 2004, Lazar and Pnevmatikakis, 2008a). These results have been extended for inputs belonging to the more general shift-invariant spaces (SIS) (Gontier and Vetterli, 2014).

System identification represents a widely used methodology for the development of quantitative models in sensory neurophysiology (Wu et al., 2006). Circuits comprising filters in series with spiking neurons have been proposed as biophysically realistic models for sensory processing systems, where the filter represents a receptive field (Lazar and Slutskiy, 2014b) or the neural dendritic tree (Lazar and Slutskiy, 2010, 2014c). The identification of such circuits was reformulated as a problem of input reconstruction for TEMs (Lazar and Slutskiy, 2013).

\subsection{Motivation}

This thesis addresses a number of information processing and modelling problems relevant to the field of computational neuroscience as well as neuromorphic systems engineering (Mead, 1990).

An important fundamental theoretical problem consists in developing mathematical formulations for the encoding and decoding of analog signals using spiking neurons. This problem was addressed by Lazar (2004), who demonstrated rigorously that bandlimited stimuli can be perfectly reconstructed from the output spike train of an IF neuron. The disadvantage of the current time encoding and decoding framework for IF neurons is that it involves formulating the encoding operation as a projection on a set of input-dependent functions. As a consequence of this formulation, the state-of-the-art algorithms reconstruct an input $u$ in a space spanned by a set of frame functions that depend on $u$. Moreover, the reconstruction algorithms are based on solving a linear system whose coefficients are different for every input function (Lazar and Pnevmatikakis, 2008a). The reconstruction of several inputs $u^{j}$ from the corresponding TEM outputs $\mathcal{T}_{u^{j}}=\left\{t_{k}^{j}\right\}_{k=1, \ldots, N+1}$ involves recalculating the frame functions and solving a new linear system for every sequence $j$. This process is computationally demanding for large values of $N$. The field would benefit from new reconstruction algorithms that overcome this computational disadvantage.

To address the issue above, a new framework is introduced for studying the encoding performed by TEMs represented by ideal IF neurons. The framework reformulates the operation performed by the IF neuron on stimulus $u$ as a problem of uniform sampling of an auxiliary function $\bar{\psi}^{\prime}$ on a set of input independent time points. Based on this framework, new algorithms are introduced for reconstructing input $u$, belonging to bandlimited as well as SIS, via the auxiliary function $\bar{\psi}^{\prime}$. The proposed approaches are significantly faster than the standard algorithms because the frame functions and the solution of the corresponding new linear system are computed off-line. 
Another important problem that is being addressed in this work is that of inferring mathematical models for sensory processing circuits directly from input-output observations. Lazar and Slutskiy (2010) proposed the problem of identifying linear filters in series with ideal IF neurons, and developed a methodology that performs full identification of the circuit. The approach has been extended to circuits where the ideal IF neuron is replaced by more biophysically realistic neuron models such as the leaky IF neuron (Lazar and Slutskiy, 2010) and the Hodgkin-Huxley model (Lazar and Slutskiy, 2014b), under the additional assumptions that the spiking neuron parameters are known or that input-output measurements of the spiking neuron are available, respectively.

Lazar and Slutskiy (2014c) extended the theoretical representation and identification framework to nonlinear filters, under the assumptions that these can be represented as a Volterra series. However, from an identification point of view, the proposed approach suffers from the well known practical limitation related to the problem of identifying Volterra kernels directly from data (Chen and Billings, 1989), namely the requirement for large amounts of data to identify higher-order kernels. In this context, the development of new methods that reduce the number of assumptions made, and enable the simultaneous identification of the spiking neuron as well as the nonlinear filter based only on a relatively small number of observations would be highly desirable.

In this respect, this thesis develops two identification methodologies for sensory processing circuits comprising a filter in cascade with a spiking neuron. The methodologies consist of two steps: the estimation of the spiking neuron parameters, and the identification of the filter.

A [Nonlinear Filter]-[Ideal IF] circuit is reformulated as a scaled nonlinear filter in series with a modified ideal IF neuron. The structure and parameters of the scaled nonlinear filter are inferred using the NARMAX system identification methodology from the reconstructed input of the neuron.

An equivalent [Linear Filter]-[Leaky IF] circuit is identified, assuming that input-output measurements of the spiking neuron are not available and that the neuron model is unknown. A new algorithm is developed for estimating the leaky IF model parameters with arbitrary precision. The structure and parameters of the scaled linear filter are inferred using the NARMAX system identification methodology from the reconstructed input of the neuron.

Ultimately, one of the biggest challenges at the moment is the lack of a general theory to characterize the processing of information by circuits that operate with spike trains, or to design circuits that implement particular processing tasks, in a similar fashion to classical analog and digital filters. Lazar (2006b) addressed the issue of computing specific mathematical operations with neural circuits. Specifically, he developed a method for realising a linear filter in the spike domain. The method performs simultaneously the computation of the filter output and the reconstruction of the spike train in analog domain. A new method 
that operates exclusively in the spike domain is desirable for the implementation of circuits that operate with spike trains.

To address this challenge, a direct relationship is developed between the time encoded input and output of an arbitrary linear filter, for a TEM represented by an ideal IF neuron. Based on this representation, a new algorithm was developed for computing the time encoded output directly from the input spike time sequence. An error bound is derived that is a function of the neuron parameters.

\subsection{Overview of the thesis}

- Chapter 2 gives an overview of encoding and decoding with TEMs in bandlimited and shift-invariant spaces. The chapter begins with a brief review of nonuniform sampling theory, which serves as a basis of foundation for the following results. The theory of encoding and decoding for TEMs in bandlimited spaces is then reviewed, where the TEMs are represented as circuits with one or several spiking neuron models. The existing results for TEMs are further discussed for inputs belonging to shift-invariant spaces. Emphasis is put in both cases on the computational disadvantage of the reconstruction methods.

- Chapter 3 introduces a novel reconstruction framework for ideal IF neurons with bandlimited inputs that aims to overcome the disadvantages of the state-of-the-art methods. The framework reformulates the input dependent nonuniform sampling operation performed by the IF neuron as an equivalent stimulus independent uniform sampling operation performed on an auxiliary function. The new result forms the basis for two new reconstruction algorithms that are significantly faster than the stateof-the-art methods. Numerical studies are performed to show the advantage of the new algorithms.

- Chapter 4 develops a novel reconstruction framework for ideal IF neurons with inputs belonging to shift-invariant spaces. Two new algorithms are introduced based on the state-of-the-art reconstruction method for shift-invariant spaces. Moreover, two algorithms are developed based on the novel framework. The computational advantage of the algorithms based on the proposed framework is shown through numerical simulations.

- Chapter 5 introduces a new approach to the identification of sensory processing circuits. A brief review is given for the current identification methodologies for circuits consisting of filters in cascade with spiking neurons. The methods reviewed show that the identification problem can be reformulated as one of stimulus reconstruction. Emphasis is put on the restrictions of the methods. The NARMAX identification 
methodology for nonlinear filters is reviewed next. Two new methods are proposed for the identification of sensory processing circuits, which identify the filter model using the NARMAX methodology. Numerical simulations are given to show the performance of both methods.

- Chapter 6 proposes the problem of determining a direct relationship between the time encoded input and output of a linear filter. The TEM is represented here by an ideal IF neuron. An algorithm is developed that computes the time encoded output directly from the input spike sequence. Through numerical simulations, it is shown that the proposed algorithm is significantly faster than the indirect method, which involves decoding the input spike time sequence, computing the analog output of the linear filter, and finally encoding the resulting output.

- Chapter 7 contains the conclusions and future work. 


\section{Chapter 2}

\section{Time encoding and decoding in bandlimited and shift-invariant spaces}

A fundamental problem in information processing is representing a continuous function as a discrete sequence of values. This problem was originally addressed in uniform sampling theory, pioneered by Shannon (1949) and Kotelnikov (1933), and nonuniform sampling theory, in the works of Beurling and Malliavin (1967) and Landau (1967).

The sampling methods based on the theories above record the amplitude value of a function at predefined time locations. A dual method, sampling based on timing, records the time location when the amplitude of a function, or an operator applied to the function, exceeds a threshold value (Gontier and Vetterli, 2014). The time encoding machine (TEM), which performs sampling based on timing, has first been defined by Lazar and Tóth (2003) as a real-time asynchronous mechanism that encodes the amplitude information of a function into a time sequence.

The TEMs have been used as replacements for classical analog-to-digital converters in signal processing applications such as brain machine interface (BMI) prototypes (Bashirullah et al., 2007) for encoding neural recordings, and human area network (HAN) prototypes for encoding biomonitoring information (Káldy et al., 2007). In both cases it was shown that the TEMs represent encoding devices with lower power and higher resolution than the classical analog-to-digital converters.

In neuroscience, the TEMs have been used for describing neuron models. One example is the integrate-and-fire (IF) neuron, which represents one of the most common models of the spiking neuron (Lapicque, 1907, Tuckwell, 1988). Lazar and Pnevmatikakis (2008a) have developed conditions under which a bandlimited input of the IF neuron can be reconstructed perfectly. The result has been extended to other TEMs, such as populations of IF 
neurons (Lazar and Pnevmatikakis, 2008a, Lazar and Slutskiy, 2014c), IF neurons with refractory period (Lazar, 2004), leaky IF (LIF) neurons (Lazar, 2005), Hodgkin-Huxley neurons (Lazar, 2010) and asynchronous sigma-delta modulators (ASDMs) (Lazar and Tóth, 2004b).

In many practical applications, the space of bandlimited functions is considered too restrictive. Under certain conditions, functions belonging to the more general shift-invariant spaces (SIS) can be perfectly reconstructed from uniform (Aldroubi et al., 1994) as well as nonuniform samples (Aldroubi and Feichtinger, 1998, Aldroubi and Gröchenig, 2001). Furthermore, Gontier and Vetterli (2014) have provided sufficient conditions for reconstructing the input of an IF neuron belonging to a SIS from the associated output sequence.

All the above reconstruction algorithms for TEMs share an important drawback. They are studied in a unifying manner with the reconstruction algorithms from nonuniform sampling theory and, in this case, the time locations of the corresponding nonuniform sampling times are input dependent, and thus different for every reconstruction. In practical applications, this causes significantly higher computational complexity than the reconstruction algorithms from uniform samples.

This chapter reviews nonuniform sampling theory in Section 2.1. The reconstruction algorithms for TEMs are presented in Section 2.2 for bandlimited spaces and in Section 2.3 for shift-invariant spaces. Conclusions are in Section 2.4.

\subsection{Nonuniform sampling and reconstruction of bandlimited functions}

Uniform sampling is studied in a unifying manner with the theory of harmonic Fourier series, as demonstrated by Shannon (1949). If the samples are not uniform in time, the reconstruction problem can be addressed using tools from frame theory (Christensen, 2003) and theory of nonharmonic Fourier series (Young, 1980). Feichtinger and Gröchenig (1994) proposed several iterative algorithms for reconstructing functions efficiently from their nonuniform samples.

In the classical sampling theory, a function $f$ can be reconstructed from the sequence of uniform samples $\{f(k T)\}_{k \in \mathbb{Z}}$, if $f \in P W_{\Omega}$ and $T=\frac{\pi}{\Omega}$, where $P W_{\Omega}$ is the Paley-Wiener space of bandwidth $\Omega$

$$
P W_{\Omega}=\left\{u \in \mathcal{L}^{2}(\mathbb{R}): \operatorname{supp}(\widehat{u}) \subseteq[-\Omega, \Omega]\right\},
$$

where $\widehat{u}$ denotes the Fourier transform of function $u$ and $\operatorname{supp}(\widehat{u})$ denotes the support of $\widehat{u}$. The space $P W_{\Omega}$ is endowed with norm $\|\cdot\|_{L^{2}}$ and inner product $\langle\cdot\rangle_{L^{2}}$. Then $f$ can be 
reconstructed as (Shannon, 1949)

$$
f(t)=\sum_{k \in \mathbb{Z}} f(k T) \cdot \operatorname{sinc}(\Omega(t-k T)) .
$$

The set of functions $\left\{\sqrt{\frac{\Omega}{\pi}} \cdot \operatorname{sinc}(\Omega(\cdot-k T))\right\}_{k \in \mathbb{Z}}$ forms an orthonormal basis on $P W_{\Omega}$, which is equivalent to the fact that $\left\{\sqrt{\frac{\pi}{\Omega}} \cdot e^{-i k T \cdot}\right\}_{k \in \mathbb{Z}}$ forms an orthonormal basis on $L^{2}[-\Omega, \Omega]$ (Naylor and Sell, 1982), also known as the harmonic Fourier basis (Aldroubi and Gröchenig, 2001), where

$$
L^{2}[-\Omega, \Omega] \triangleq\left\{f:[-\Omega, \Omega] \rightarrow \mathbb{C}: \int_{-\infty}^{\infty}|f(\omega)|^{2} d \omega<\infty\right\}
$$

The following definition was presented in (Gröchenig and Razafinjatovo, 1996) for characterising a nonuniform sequence of sampling times $\left\{x_{k}\right\}_{k \in \mathbb{Z}}$.

Definition 2.1. A sequence $\left\{x_{k}\right\}_{k \in \mathbb{Z}}$ is called a set of sampling for $P W_{\Omega}$ if there exist $A, B>0$ such that

$$
A\|f\|_{L^{2}}^{2} \leq \sum_{k \in \mathbb{Z}}\left|f\left(x_{k}\right)\right|^{2} \leq B\|f\|_{L^{2}}^{2}, \forall f \in P W_{\Omega} .
$$

This is also equivalent to the fact that $\left\{g_{\Omega}\left(\cdot-x_{k}\right)\right\}_{k \in \mathbb{Z}}$ is a frame on $P W_{\Omega}$, where $g_{\Omega}=\frac{\sin (\Omega \cdot)}{\pi \cdot}$ is the reproducing kernel of $P W_{\Omega}$. If this is true, then any function $f \in P W_{\Omega}$ can be reconstructed from its samples at points $\left\{x_{k}\right\}_{k \in \mathbb{Z}}$, which satisfy

$$
f\left(x_{k}\right)=\left\langle f, g_{\Omega}\left(\cdot-x_{k}\right)\right\rangle_{L^{2}}, \forall k \in \mathbb{Z} \text {. }
$$

Lazar and Pnevmatikakis (2008a) have proven that $\left\{g_{\Omega}\left(\cdot-x_{k}\right)\right\}_{k \in \mathbb{Z}}$ is a frame on $P W_{\Omega}$ if and only if $\left\{e^{-i x_{k} \cdot}\right\}_{k \in \mathbb{Z}}$ is a frame for $L^{2}[-\Omega, \Omega]$, i.e.,

$$
A\|f\|_{L_{\Omega}^{2}}^{2} \leq \sum_{k \in \mathbb{Z}}\left|\left\langle f, e^{-i x_{k} \cdot}\right\rangle_{L_{\Omega}^{2}}\right|^{2} \leq B\|f\|_{L_{\Omega}^{2}}^{2}, \forall f \in L^{2}[-\Omega, \Omega],
$$

where $\left\langle f_{1}, f_{2}\right\rangle_{L_{\Omega}^{2}} \triangleq \frac{1}{2 \pi} \int_{-\Omega}^{\Omega} f_{1}(\omega) f_{2}^{*}(\omega) d \omega,\|f\|_{L_{\Omega}^{2}} \triangleq \sqrt{\langle f, f\rangle_{L_{\Omega}^{2}}}$ are the inner product and norm on $L^{2}[-\Omega, \Omega]$, respectively, and $f_{2}^{*}$ denotes the conjugate of complex function $f_{2}$. By substituting $f$ with $f^{*}$ in (2.2) and using the properties of the inner product, it follows that this is also equivalent to the fact that $\left\{e^{i x_{k} \cdot}\right\}_{k \in \mathbb{Z}}$ is a frame on $L^{2}[-\Omega, \Omega]$. The latter is one of the main problems of interest in the theory of nonharmonic Fourier series (Young, 1980). The main results that establish conditions for the existence of frames on $L^{2}[-\Omega, \Omega]$ are presented as follows. The review is limited to countable sequences of reals $\left\{x_{k}\right\}_{k \in \mathbb{Z}}$.

Definition 2.2. Sequence $\left\{x_{k}\right\}_{k \in \mathbb{Z}}$ is called relatively separated if $\exists \zeta>0$ such that $\mid x_{n}-$ 
$x_{k} \mid>\zeta, \forall n, k \in \mathbb{Z}, n \neq k$.

Definition 2.3. A relatively separated sequence $X=\left\{x_{k}\right\}_{k \in \mathbb{Z}}$ is uniformly dense with uniform density $d(X)$ if

$$
\exists L>0, \forall k \in \mathbb{Z},\left|x_{k}-\frac{k}{d(X)}\right| \leq L
$$

The following theorem was proven by Duffin and Schaeffer (1952).

Theorem 2.1 (Duffin and Schaeffer). Let $X=\left\{x_{k}\right\}_{k \in \mathbb{Z}}$ be a uniformly dense sequence with uniform density $d(X)$. Then $\left\{e^{i x_{k} \cdot}\right\}_{k \in \mathbb{Z}}$ is a frame for $L^{2}[-\Omega, \Omega]$ provided that $0<$ $\frac{\Omega}{\pi}<d(X)$.

For relatively separated sequences that are not uniformly dense, Landau (1967) established the following condition for the existence of a frame on $L^{2}[-\Omega, \Omega]$.

Theorem 2.2 (Landau). Let $X=\left\{x_{k}\right\}_{k \in \mathbb{Z}}$ be a relatively separated sequence. Then the lower uniform density of $X$, defined as

$$
D^{-}(X) \triangleq \lim _{r \rightarrow \infty} \frac{\inf _{x \in \mathbb{R}} \operatorname{card}(X \cap[x, x+r])}{r},
$$

always exists. Moreover, if $D^{-}(X)>\frac{\Omega}{\pi}$, then $\left\{e^{i x_{k} \cdot}\right\}_{k \in \mathbb{Z}}$ is a frame for $L^{2}[-\Omega, \Omega]$.

The lower density of a relatively separated sequence $X$ that is uniformly dense is $D^{-}(X)=$ $d(X)$ (Jaffard, 1991). The following is an alternative definition for the density of a sequence.

Definition 2.4. Sequence $\left\{x_{k}\right\}_{k \in \mathbb{Z}}$ is called $\Delta$ - dense if $\left|x_{k+1}-x_{k}\right| \leq \Delta, \forall k \in \mathbb{Z}$.

A $\Delta$ - dense sequence $X=\left\{x_{k}\right\}_{k \in \mathbb{Z}}$ that is relatively separated has a lower density that is greater or equal to that of $Y=\{k \Delta\}_{k \in \mathbb{Z}}$, for which $D^{-}(Y)=\frac{1}{\Delta}$. It follows that Theorem 2.2 can be applied to sequence $X$ provided that $\Delta<\frac{\pi}{\Omega}$, which was proven explicitly in the following theorem (Benedetto, 1992).

Theorem 2.3 (Benedetto). Let $\left\{x_{k}\right\}_{k \in \mathbb{Z}}$ be a strictly increasing, relatively separated, $\Delta$ dense sequence. Then $\left\{e^{i x_{k} \cdot}\right\}_{k \in \mathbb{Z}}$ is a frame for $L^{2}[-\Omega, \Omega]$ provided that $\Delta<\frac{\pi}{\Omega}$.

A function can be reconstructed from its nonuniform samples $\left\{x_{k}\right\}_{k \in \mathbb{Z}}$, provided that $\left\{g_{\Omega}\left(\cdot-x_{k}\right)\right\}_{k \in \mathbb{Z}}$ is a frame on $P W_{\Omega}$, using the frame expansion or the dual frame expansion presented in Appendix A. In practice, this involves calculating the dual frame, which is a challenging task for generic nonuniform sequences.

Feichtinger and Gröchenig (1994) have developed an efficient iterative algorithm for reconstructing a function from its nonuniform samples with arbitrary accuracy. 
Theorem 2.4. Let $\left\{x_{k}\right\}_{k \in \mathbb{Z}}$ be a $\Delta$-dense sequence and let

$$
\mathcal{V}: P W_{\Omega} \rightarrow P W_{\Omega}, \mathcal{V} f=\mathcal{P}_{P W_{\Omega}}\left(\sum_{k \in \mathbb{Z}} f\left(x_{k}\right) 1_{\left[y_{k-1}, y_{k}[\right.}\right),
$$

where $\mathcal{P}_{P W_{\Omega}}: L^{2}(\mathbb{R}) \rightarrow P W_{\Omega}$ is the orthogonal projection operator on $P W_{\Omega}, y_{k} \triangleq$ $\frac{x_{k}+x_{k+1}}{2}$, and $1_{\left[y_{k-1}, y_{k}[\right.}$ is the characteristic function of interval $\left[y_{k-1}, y_{k}[, \forall k \in \mathbb{Z}\right.$. Provided that $\Delta<\frac{\pi}{\Omega}$, any function $f \in P W_{\Omega}$ can be reconstructed iteratively from its samples $\left\{f\left(x_{k}\right)\right\}_{k \in \mathbb{Z}}$ as follows

$$
\begin{aligned}
f_{0} & =\mathcal{V} f \\
f_{n+1} & =f_{n}+\mathcal{V}\left(f-f_{n}\right), \forall n \geq 0 .
\end{aligned}
$$

Then $\lim _{n \rightarrow \infty} f_{n}=f$ and

$$
\left\|f-f_{n}\right\|_{L^{2}} \leq\left(\frac{\Delta \Omega}{\pi}\right)^{n+1}\|f\|_{L^{2}}, \forall n \in \mathbb{N}
$$

\subsection{Time encoding and decoding in bandlimited spaces}

The review in this chapter is focused on the time encoding machine (TEM), which is a mechanism performing sampling based on timing, the dual of nonuniform sampling. The TEM is defined mathematically as an operator $\mathscr{T}: P W_{\Omega} \rightarrow \mathbb{R}^{\mathbb{Z}}$ that maps a function $u \in$ $P W_{\Omega}$ to a strictly increasing sequence of reals $\mathscr{T} u=\left\{t_{k}\right\}_{k \in \mathbb{Z}}$ satisfying $\lim _{k \rightarrow \pm \infty} t_{k}=$ $\pm \infty$. If operator $\mathscr{T}$ is invertible, then $\mathscr{T}^{-1}: \mathbb{R}^{\mathbb{Z}} \rightarrow P W_{\Omega}$ is called a time decoding machine (TDM).

The TEMs arise in neuroscience as models of spiking neurons, called integrate-and-fire (IF) neurons (Dayan and Abbott, 2003). Reconstruction algorithms have been designed for the bandlimited inputs of linear (Lazar and Pnevmatikakis, 2008a) as well as nonlinear (Lazar and Slutskiy, 2014c) filter banks in cascade with ensembles of ideal IF neurons, ideal IF neurons with refractory period (Lazar, 2004), leaky IF (LIF) neurons (Lazar, 2005), populations of LIF neurons with random thresholds (Lazar et al., 2010), as well as populations of Hodgkin-Huxley neurons (Lazar, 2007, 2010). Florescu and Coca (2015) have introduced a new reconstruction algorithm for ideal IF neurons that redefines IF time encoding as a uniform sampling problem.

The asynchronous sigma-delta modulator (ASDM) (Roza, 1997), an alternative to the common analog to digital converters, is an example of a nonlinear circuit that can be modelled with a TEM. Lazar and Tóth (2004b) have developed an iterative algorithm that recon- 
structs the bandlimited input of an ASDM with arbitrary accuracy from its output sequence and demonstrated the strong relationship between TEMs and nonuniform sampling.

This section reviews the reconstruction algorithms developed for the above TEMs for bandlimited stimuli.

\subsubsection{The ideal IF neuron}

The ideal IF neuron, depicted in Figure 2.1, consists of an adder and an ideal integrator. Each time the integrator output reaches a threshold value $\delta$, the neuron fires and the integrator is reset.

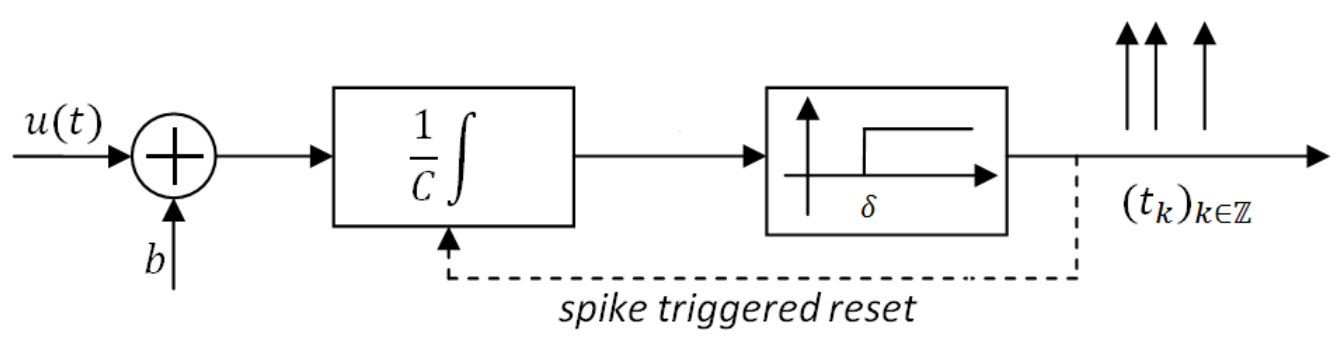

Figure 2.1: The ideal IF neuron.

Definition 2.5. An ideal IF neuron generates output spike sequence $\left\{t_{k}\right\}_{k \in \mathbb{Z}}$ when presented with input $u$, satisfying $|u| \leq c<b$, such that

$$
\int_{t_{k}}^{t_{k+1}} u(\tau) d \tau=C \delta-b\left(t_{k+1}-t_{k}\right), \forall k \in \mathbb{Z}
$$

where $\delta, C$, and $b$ represent the threshold, integration constant, and bias, respectively. Without reducing the generality, it is assumed that $t_{1}=0$.

Equation (2.3) is known as the $t$-transform of the ideal IF neuron. Lazar and Pnevmatikakis (2008a) have proven that for any input $u \in P W_{\Omega}$ the $t$ - transform can be expressed as

$$
\mathcal{L}_{k}^{\mathcal{T}_{u}} u=\left\langle u, \phi_{k}^{\mathcal{T}_{u}}\right\rangle_{L^{2}}=q_{k}, \forall k \in \mathbb{Z}
$$

where $\phi_{k}^{\mathcal{T}_{u}} \triangleq g_{\Omega} * 1_{\left[t_{k}, t_{k+1}[\right.}$, and $1_{\left[t_{k}, t_{k+1}[\right.}$ is the characteristic function of interval $\left[t_{k}, t_{k+1}[, \forall k \in\right.$ $\mathbb{Z}$.

Therefore, the $t$ - transform represents the orthogonal projection of $u$ on functions $\left\{\phi_{k}^{\mathcal{T}_{u}}\right\}_{k \in \mathbb{Z}}$. As in nonuniform sampling theory, where the samples at points $\left\{x_{k}\right\}_{k \in \mathbb{Z}}$ represent the projection of a continuous function on $\left\{g_{\Omega}\left(\cdot-x_{k}\right)\right\}_{k \in \mathbb{Z}}$, an important problem in 
encoding with ideal IF neurons is establishing conditions for which $\left\{\phi_{k}^{\mathcal{T}_{u}}\right\}_{k \in \mathbb{Z}}$ forms a frame on $P W_{\Omega}$. The following lemma addresses this issue (Lazar and Pnevmatikakis, 2008a).

Lemma 2.1. Let $\mathcal{T}=\left\{t_{k}\right\}_{k \in \mathbb{Z}}$ be a relatively separated sequence of reals. Then sequences $\left\{g_{\Omega}\left(\cdot-s_{k}\right)\right\}_{k \in \mathbb{Z}}$ and $\left\{\phi_{k}^{\mathcal{T}}\right\}_{k \in \mathbb{Z}}$ are frames on $P W_{\Omega}$ provided that $D^{-}(\mathcal{T})>\frac{\Omega}{\pi}$, where $s_{k} \triangleq \frac{t_{k-1}+t_{k}}{2}$ and $D^{-}(\mathcal{T})$ represents the lower density of sequence $\mathcal{T}$

$$
D^{-}(\mathcal{T}) \triangleq \lim _{r \rightarrow \infty} \frac{\inf _{x \in \mathbb{R}} \operatorname{card}(\mathcal{T} \cap[x, x+r])}{r} .
$$

For a sequence $\mathcal{T}_{u}$, generated by an ideal IF neuron with parameters $\{b, \bar{\delta}\}$ when presented with input $u \in P W_{\Omega}$, Lazar and Pnevmatikakis (2008a) have shown that $D^{-}\left(\mathcal{T}_{u}\right) \geq$ $\frac{b-c}{\delta}$, and later on that $D^{-}\left(\mathcal{T}_{u}\right)=\frac{b}{\delta}$ (Lazar and Pnevmatikakis, 2011). Furthermore, $\mathcal{T}_{u}$ is relatively separated as it satisfies (Lazar, 2004)

$$
\frac{\bar{\delta}}{b+c} \leq t_{k+1}-t_{k} \leq \frac{\bar{\delta}}{b-c}, \forall k \in \mathbb{Z}
$$

If $\frac{b}{\delta}>\frac{\Omega}{\pi}$, due to Lemma 2.1 , input $u$ can be represented as

$$
u(t)=\sum_{k \in \mathbb{Z}} c_{k} g_{\Omega}\left(t-s_{k}\right), \forall t \in \mathbb{R}
$$

Coefficients $\left\{c_{k}\right\}_{k \in \mathbb{Z}}$ satisfy (Lazar and Pnevmatikakis, 2008a)

$$
c=G^{+} \boldsymbol{q}
$$

where $G^{+}$denotes the Moore-Penrose pseudoinverse of matrix $G,[\boldsymbol{c}]_{k}=c_{k},[\boldsymbol{q}]_{k}=\bar{\delta}-$ $b\left(t_{k+1}-t_{k}\right)$, and $[G]_{k, l}=\int_{t_{k}}^{t_{k+1}} g_{\Omega}\left(\tau-s_{l}\right) d \tau, \forall k, l \in \mathbb{Z}$.

The reconstruction of bandlimited inputs is only possible if the IF neuron parameters satisfy $\frac{b}{\delta}>\frac{\Omega}{\pi}$. If this is not valid, Lazar and Pnevmatikakis (2008a) proved that a bandlimited input can still be recovered from a sufficiently large population of IF neurons. Moreover, they designed a model that arises in several sensory systems, consisting of a bank of filters in cascade with a population of IF neurons, depicted in Figure 2.2. The filters, which model the processing taking place in the dendritic trees of biological neurons, are required to satisfy the following stability property.

Definition 2.6. A linear filter $h$ is called bounded-input bounded-output (BIBO) stable if

$$
\int_{-\infty}^{\infty}|h(\tau)| d \tau<\infty .
$$




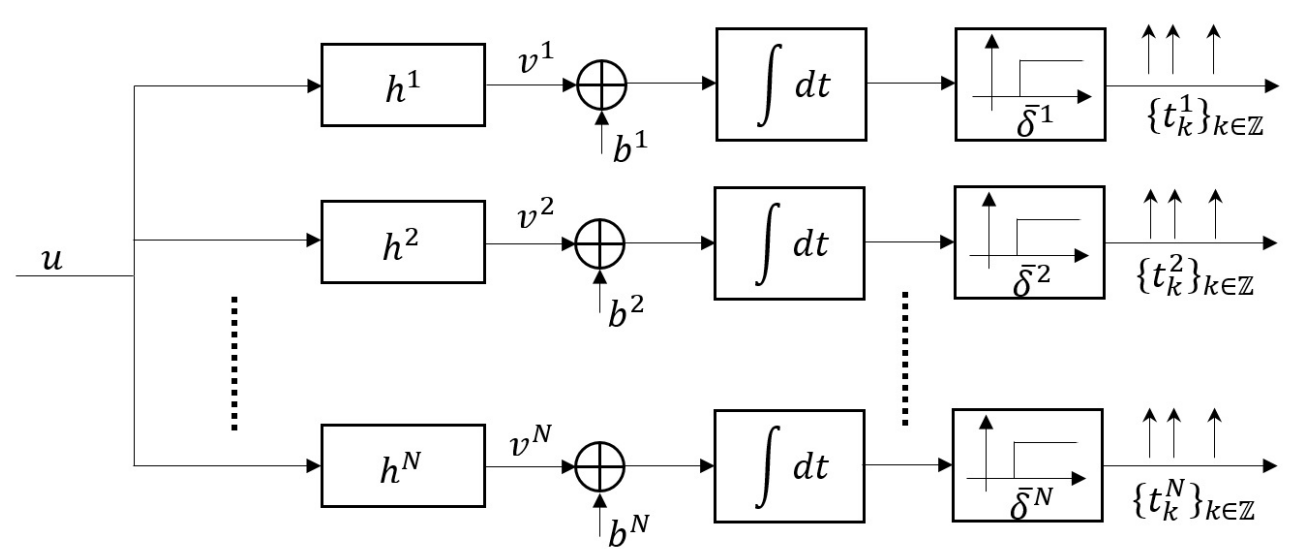

Figure 2.2: Single-input multi-output population of $N$ ideal IF neurons.

The $t$-transform of the population of IF neurons satisfies

$$
\int_{t_{k}^{j}}^{t_{k+1}^{j}}\left(h^{j} * u\right)(\tau) d \tau=q_{k}^{j}, \forall k \in \mathbb{Z}, \forall j=1, \ldots, N,
$$

where $q_{k}^{j}=\bar{\delta}^{j}-b^{j}\left(t_{k+1}^{j}-t_{k}^{j}\right), \forall k \in \mathbb{Z}, \bar{\delta}^{j}=C^{j} \delta^{j}$ and $C^{j}, \delta^{j}$ denote the integration constant and threshold of neuron $j, \forall j=1, \ldots, N$. To prevent the situation in which different neurons trigger the same spike times, i.e., some neurons don't encode any new information, the filters are required to satisfy the following property (Lazar and Pnevmatikakis, 2008a).

Definition 2.7. The filters $\left\{h^{j}\right\}_{j=1, \ldots, N}$ are called linearly independent if $\nexists a_{j}, j=1, \ldots, N$, not all zero, and real numbers $\alpha^{j}, j=1, \ldots, N$, such that

$$
\sum_{j=1}^{N} a_{j}\left(h_{j} * g_{\Omega}\right)\left(\cdot-\alpha_{j}\right)=0 \text { a.e. }
$$

Let $\mathcal{T}_{u}^{j} \triangleq\left\{t_{k}^{j}\right\}_{k \in \mathbb{Z}}, \forall j=1, \ldots, N$. Then equation (2.8) is equivalent to (Lazar and Pnevmatikakis, 2008a)

$$
\left\langle u, \phi_{k, j}^{\mathcal{T}_{u}^{j}}\right\rangle_{L^{2}}=q_{k^{\prime}}^{j}, \forall k \in \mathbb{Z}, \forall j=1, \ldots, N,
$$

where $\phi_{k, j}^{\mathcal{T}_{u}^{j}} \triangleq \widetilde{h}^{j} * g_{\Omega} * 1_{\left[t_{k}^{j}, t_{k+1}^{j}\right.}$ and $\widetilde{h}^{j} \triangleq h^{j}(-\cdot), \forall k \in \mathbb{Z}, \forall j=1, \ldots, N$.

Let $\mathcal{T}_{u} \triangleq \underset{j=1, \ldots, N}{\bigcup} \mathcal{T}_{u}^{j}$ and $\psi_{k}^{j} \triangleq\left(\widetilde{h}^{j} * g_{\Omega}\right)\left(\cdot-s_{k}\right), \forall k \in \mathbb{Z}, \forall j=1, \ldots, N$. Lazar and Pnevmatikakis (2008a) have proven that sequences $\left\{\psi_{k}^{j}\right\}_{k \in \mathbb{Z}, j=1, \ldots, N}$ and $\left\{\phi_{k, j}^{\mathcal{T}_{u}^{j}}\right\}_{k \in \mathbb{Z}, j=1, \ldots, N}$ 
are both frames on $P W_{\Omega}$ provided that

$$
\sum_{j=1}^{N} \frac{1}{\bar{\delta}^{j}}\left(b^{j}-c \int_{-\infty}^{\infty}\left|h^{j}(\tau)\right| d \tau\right)>\frac{\Omega}{\pi}
$$

and thus input $u$ can be recovered as

$$
u=\sum_{j=1}^{N} \sum_{k \in \mathbb{Z}} c_{k}^{j} \psi_{k}^{j}
$$

Coefficients $\left\{c_{k}^{j}\right\}_{k \in \mathbb{Z}, j=1, \ldots, N}$ satisfy (Lazar and Pnevmatikakis, 2008a)

$$
c=G^{+} \boldsymbol{q}
$$

where $\boldsymbol{c}=\left[\boldsymbol{c}^{1}, \ldots, \boldsymbol{c}^{N}\right]^{T},\left[\boldsymbol{c}^{j}\right]_{k}=c_{k^{\prime}}^{j} \boldsymbol{q}=\left[\boldsymbol{q}^{1}, \ldots, \boldsymbol{q}^{N}\right]^{T},\left[\boldsymbol{q}^{j}\right]_{k}=q_{k}^{j}$, and

$$
\boldsymbol{G}=\left(\begin{array}{ccc}
\boldsymbol{G}^{11} & \ldots & \boldsymbol{G}^{1 N} \\
\ldots & & \ldots \\
G^{N 1} & \ldots & \boldsymbol{G}^{N N}
\end{array}\right),\left[\boldsymbol{G}^{n j}\right]_{k, l}=\int_{t_{k}^{n}}^{t_{k+1}^{n}}\left(h^{n} * \widetilde{h}^{j} * g\right)\left(\tau-s_{l}^{j}\right) d \tau .
$$

The reconstruction methodology was further extended to three dimensional inputs, representing an analog monochromatic video stream (Lazar and Pnevmatikakis, 2008b), and to multiple inputs of different dimensions (Lazar and Slutskiy, 2013).

Lazar and Slutskiy (2014c) have shown that the dendritic computations in biological neurons can be modelled more accurately with nonlinear filters. The paper developed an algorithm that reconstructs perfectly the inputs of a multi-input multi-output circuit consisting of a bank of nonlinear filters in cascade with a population of ideal IF neurons.

\subsubsection{The ideal IF neuron with refractory period}

The ideal IF neuron with refractory period represents an extension of the ideal IF neuron, incorporating the absolute refractory period of a biological neuron (Lazar, 2004).

Definition 2.8. The ideal IF neuron with refractory period generates the output sequence of spike times $\left\{t_{k}\right\}_{k \in \mathbb{Z}}$ when presented with input $u$, satisfying $|u| \leq c<b$, such that

$$
\int_{t_{k}+\Delta_{r}}^{t_{k+1}} u(\tau) d \tau=\bar{\delta}-b\left(t_{k+1}-t_{k}-\Delta_{r}\right), \forall k \in \mathbb{Z}
$$

where $\Delta_{r}$ represents the absolute refractory period and $\bar{\delta}=C \delta$. 
Lazar (2004) has proven that $\Delta_{r}$ does not lead to information loss, and that any input $u \in P W_{\Omega}$ can be reconstructed perfectly from spike times $\left\{t_{k}\right\}_{k \in \mathbb{Z}}$ provided that

$$
\frac{\bar{\delta}}{b-c} \cdot \frac{\Omega}{\pi}<\frac{1-\epsilon}{1+\epsilon}
$$

where $\epsilon=\sqrt{\frac{\Delta_{r}}{\delta /(b+c)+\Delta_{r}}}$. Function $u$ is recovered as

$$
u=g G^{+} q
$$

where $[\boldsymbol{g}]_{1, l}=g_{\Omega}\left(\cdot-s_{l}\right),[\boldsymbol{G}]_{k, l}=\int_{t_{k}+\Delta_{r}}^{t_{k+1}} g_{\Omega}\left(\tau-s_{l}\right) d \tau$, and $[\boldsymbol{q}]_{k, 1}=\bar{\delta}-b\left(t_{k+1}-t_{k}-\right.$ $\left.\Delta_{r}\right), \forall l, k \in \mathbb{Z}$.

\subsubsection{The leaky IF neuron}

The leaky IF (LIF) neuron is a TEM that consists of an adder in cascade with a linear $R C$ filter. The neuron triggers a spike each time the filter output $y$ reaches a threshold value $\delta$.

Definition 2.9. The LIF neuron generates the output sequence of spike times $\left\{t_{k}\right\}_{k \in \mathbb{Z}}$ when presented with input $u$, satisfying $|u| \leq c<b$, such that

$$
\int_{t_{k}}^{t_{k+1}} u(\tau) e^{-\frac{t_{k+1}-\tau}{R C}} d \tau=C(\delta-b R)+C\left(b R-y\left(t_{0}\right)\right) e^{-\frac{t_{k+1}-t_{k}}{R C}}
$$

where $b$ is the bias and $y\left(t_{0}\right)$ is the initial condition of the $R C$ filter.

The inputs $u$ that additionally satisfy $u \in P W_{\Omega}$ can be recovered from spike times $\left\{t_{k}\right\}_{k \in \mathbb{Z}}$ provided that (Lazar, 2005)

$$
R C \cdot \ln \left(1-\frac{\delta-y\left(t_{0}\right)}{\delta-(b-c) R}\right) \frac{\Omega}{\pi}<\frac{1-\epsilon^{\prime}}{1+\epsilon^{\prime}}
$$

where $\epsilon^{\prime}=\frac{\delta-y\left(t_{0}\right)}{(b-c) R-y\left(t_{0}\right)}$. Function $u$ is recovered as

$$
u=g G^{+} q
$$

where $[\boldsymbol{g}]_{1, l}=g_{\Omega}\left(\cdot-s_{l}\right),[\boldsymbol{G}]_{k, l}=\int_{t_{k}}^{t_{k+1}} g_{\Omega}\left(\tau-s_{l}\right) e^{-\frac{t_{k+1}-\tau}{R C}} d \tau$, and $[\boldsymbol{q}]_{k, 1}=C(\delta-b R)+$ $C\left(b R-y\left(t_{0}\right)\right) e^{-\frac{t_{k+1}-t_{k}}{R C}}$.

Alternative reconstruction algorithms have been proposed for the input $u$ of a LIF neuron on a finite time horizon (Lazar and Pnevmatikakis, 2009), i.e., $u \in L^{2}[0, T]$ and $\mathcal{T}_{u}=\left\{t_{k}\right\}_{k=1, \ldots, N}$. Instead of perfect reconstruction, the paper proposes an algorithm that is consistent, i.e., the reconstructed function generates exactly the same spike train $\mathcal{T}_{u}$ as the original input function. An extra constraint imposed on the reconstructed signal is to 
minimize the following cost function

$$
\mathcal{C} u=\left[\int_{0}^{T}\left(\frac{d^{2} u}{d t^{2}}(\tau)\right)^{2} d \tau\right]^{1 / 2}
$$

Function $u$ is reconstructed using the following theorem, presented in (Lazar and Pnevmatikakis, 2009).

Theorem 2.5. The consistent reconstruction, optimal with respect to cost function $\mathcal{C} u$, is unique, and has the expression

$$
u_{o p t}(t)=d_{0}+d_{1} x+\sum_{k=1}^{N-1} c_{k} \xi_{k}(x)
$$

where

$$
\xi_{k}(t)=\int_{t_{k}}^{t_{k+1}}|t-s|^{3} e^{-\frac{t_{k+1}-s}{R C}} d s,
$$

where $|\cdot|$ denotes the absolute value. Coefficients $d_{0}, d_{1}$ and $c_{k}, k=1,2, \ldots, N-1$, are the solution of the following linear system:

$$
\left[\begin{array}{ccc}
\boldsymbol{G} & \boldsymbol{p} & \boldsymbol{r} \\
\boldsymbol{p}^{T} & 0 & 0 \\
\boldsymbol{r}^{T} & 0 & 0
\end{array}\right]\left[\begin{array}{c}
\boldsymbol{c} \\
d_{0} \\
d_{1}
\end{array}\right]=\left[\begin{array}{l}
\boldsymbol{q} \\
0 \\
0
\end{array}\right],
$$

where $\boldsymbol{c}=\left[c_{1}, c_{2}, \ldots, c_{N-1}\right]^{T}$, and $\boldsymbol{q}=\left[q_{1}, q_{2}, \ldots, q_{N-1}\right]^{T}$. Matrix $[\boldsymbol{G}]_{(N-1) \times(N-1)}$ and column vectors $\boldsymbol{p}$ and $\boldsymbol{r}$ have the following expressions:

$$
\begin{aligned}
{[\boldsymbol{p}]_{k} } & =<\phi_{k}, 1>_{L_{T}^{2}}, \\
{[\boldsymbol{r}]_{k} } & =<\phi_{k}, t>_{L_{T}^{2}} \\
{[\boldsymbol{G}]_{k l} } & =<\phi_{k}, \zeta_{l}>_{L_{T}^{2}}
\end{aligned}
$$

where $\left\langle\cdot, \cdot>_{L_{T}^{2}}\right.$ is the standard inner product on $L^{2}[0, T],<f, g>_{L_{T}^{2}}=\int_{0}^{T} f(\tau) g^{*}(\tau) d \tau$, $\forall f, g \in L^{2}[0, T]$, and $\phi_{k} \triangleq e^{-\frac{t_{k+1}-\dot{R}}{R C}} \cdot 1_{\left[t_{k}, t_{k+1}\right]}$.

\subsubsection{The leaky IF neuron with random threshold}

The LIF neuron with random threshold has been proposed as a model that incorporates the variability in the biological spiking neurons (Lazar et al., 2010).

Definition 2.10. The LIF neuron with random threshold generates a spike time sequence $\left\{t_{k}\right\}_{k=1, \ldots, N}$ when presented with input $u$, satisfying $|u| \leq c<b$, such that

$$
\int_{t_{k}}^{t_{k+1}} u(\tau) e^{-\frac{t_{k+1}-\tau}{R C}} d \tau=q_{k}+\epsilon_{k}, k=1, \ldots,(N-1),
$$


where $q_{k} \triangleq C \delta-b R C\left(1-e^{-\frac{t_{k+1}-t_{k}}{R C}}\right), \epsilon_{k} \triangleq C\left(\delta_{k}-\delta\right)$ and $\delta_{k}$ is the random threshold drawn from the normal distribution with zero mean and variance $(C \sigma)^{2}$, for $k=$ $1, \ldots,(N-1)$.

Lazar et al. (2010) have considered the reconstruction problem for periodic inputs $u \in$ $\mathcal{H}_{\Omega}^{M}$. They have proven that, in this case, equation (2.14) is equivalent to

$$
\left\langle u, \chi_{k}\right\rangle=q_{k}+\epsilon_{k}
$$

where $\left\{\chi_{k}\right\}_{k=1, \ldots,(N-1)}$ can be calculated using the orthonormal basis $\left\{e_{m}\right\}_{m=-M, \ldots, M}$, $e_{m} \triangleq \frac{1}{\sqrt{T}} e^{j m \frac{\Omega}{M} t}, m=-M, \ldots, M$ as

$$
\chi_{k}=\sum_{-M}^{M} b_{m, k} e_{m}, \forall k=1, \ldots,(N-1)
$$

where

$$
\begin{aligned}
b_{m, k} & \triangleq \frac{R C \cdot e_{-m}\left(t_{k+1}\right)+\left(y_{k}-R C\right) e_{-m}\left(t_{k}\right)}{\sqrt{T}(1-j m R C \cdot \Omega / M)}, \forall m=-M, \ldots, M, \\
y_{k} & \triangleq R C\left(1-e^{-\frac{t_{k+1}-t_{k}}{R C}}\right), \forall k=1, \ldots,(N-1) .
\end{aligned}
$$

The reconstruction $u_{o p t}$ is calculated as the solution to the minimization problem

$$
u_{\text {opt }}=\underset{u \in \mathcal{H}_{\Omega}^{M}}{\operatorname{argmin}}\left(\sum_{k=1}^{N-1}\left(q_{k}-\left\langle u, \chi_{k}\right\rangle_{\mathcal{H}_{\Omega}^{M}}\right)+(N-1) \mu\|u\|_{\mathcal{H}_{\Omega}^{M}}^{2}\right) \text {, }
$$

where $\mu$ is a positive parameter that regulates the tradeoff between smoothness and the faithfulness of measurements.

Lazar et al. (2010) have proven that function $u_{o p t}$ satisfies

$$
u_{o p t}=e \cdot c
$$

where $[\boldsymbol{e}]_{1}=e_{m},[\boldsymbol{c}]_{m}=c_{m}, \forall m=-M, \ldots, M$, are line and column vectors, respectively, and

$$
\boldsymbol{c}=\left(\boldsymbol{G}^{H} \boldsymbol{G}+(N-1) \mu \boldsymbol{I}\right)^{-1} \boldsymbol{G}^{H} \boldsymbol{q},
$$

where $[\boldsymbol{G}]_{k m}=b_{m, k}^{*},[\boldsymbol{q}]_{k}=q_{k}, k=1, \ldots,(N-1), m=-M, \ldots, M$, and $\boldsymbol{I}$ is the identify matrix of dimension $(2 M+1) \times(2 M+1)$. 


\subsubsection{The Hodgkin-Huxley neuron}

The Hodgkin-Huxley neuron is one of the best known biophysically realistic models for the spiking neuron introduced by Hodgkin and Huxley (1952).

Definition 2.11. The Hodgkin-Huxley neuron generates output function $V$ when presented with input I such that

$$
\begin{aligned}
C \frac{d V}{d t} & =-g_{N a} m^{3} h\left(V-E_{N a}\right)-g_{K} n^{4}\left(V-E_{K}\right)-g_{L}\left(V-E_{L}\right)+I \\
\frac{d m}{d t} & =\alpha_{m}(V)(1-m)-\beta_{m}(V) m \\
\frac{d h}{d t} & =\alpha_{H}(V)(1-h)-\beta_{h}(V) h \\
\frac{d n}{d t} & =\alpha_{n}(V)(1-n)-\beta_{n}(V) n .
\end{aligned}
$$

In the definition above $V$ represents the membrane voltage, i.e., the difference in electric potential between the interior and the exterior of the neuron and $m, h$, and $n$ are the gating variables. The gating variables model the conductance (resistance ${ }^{-1}$ ) of the corresponding ion channel, which is a membrane protein that establishes a resting membrane potential of the neuron.

The Hodgkin-Huxley equations can be expressed in matrix form as

$$
\frac{d x}{d t}=f(x)
$$

where $x=[V, m, h, n]^{T}$ and $f: \mathbb{R}^{4} \rightarrow \mathbb{R}^{4}$ has an expression given by the system of equations above.

A Hodgkin-Huxley neuron stimulated via multiplicative coupling by input function $u$ is described by (Lazar, 2007)

$$
\frac{d \boldsymbol{y}}{d t}=(b+u(t)) \boldsymbol{f}(\boldsymbol{y}),
$$

where $b$ is a constant satisfying $u(t)+b>0, \forall t \in \mathbb{R}$, and $\boldsymbol{y}=\left[y_{1}, y_{2}, y_{3}\right]^{T}$. The solution to system (2.16) is $y(t)=x\left(b t+\int_{0}^{t} u(\tau) d \tau\right)$, where $x$ is the solution to (2.15). The spiking times of the Hodgkin-Huxley neuron, denoted $\left\{\delta_{k}\right\}_{k \in \mathbb{Z}}$, are defined as the local maxima of function $[x]_{1}=V$. Similarly, the spike times of the Hodgkin-Huxley neuron with multiplicative coupling $\left\{t_{k}\right\}_{k \in \mathbb{Z}}$ are the local maxima of function $[\boldsymbol{y}]_{1}=y_{1}$ (Lazar, 2010).

The following lemma proves that a Hodgkin-Huxley neuron with multiplicative coupling and an ideal IF neuron with variable threshold sequence $\left\{\delta_{k+1}-\delta_{k}\right\}_{k \in \mathbb{Z}}$ are inputoutput equivalent, i.e., they both trigger the same spike sequence $\left\{t_{k}\right\}_{k \in \mathbb{Z}}$ when presented with the same input $u$ (Lazar, 2010). 
Lemma 2.2. The spike times $\left\{\delta_{k}\right\}_{k \in \mathbb{Z}}$ and $\left\{t_{k}\right\}_{k \in \mathbb{Z}}$ satisfy

$$
\int_{t_{k}}^{t_{k+1}} u(\tau) d \tau=\delta_{k+1}-\delta_{k}-b\left(t_{k+1}-t_{k}\right), \forall k \in \mathbb{Z}
$$

Lazar (2010) has shown that, under the assumption $\delta_{k+1}-\delta_{k}=\delta, \forall k \in \mathbb{Z}$, a bandlimited input $u \in P W_{\Omega}$ of a Hodgkin-Huxley neuron with multiplicative coupling can be perfectly reconstructed from the generated output spike sequence provided that $\delta / b<\pi / \Omega$.

The Hodgkin-Huxley neuron with multiplicative coupling belongs to a more general class of models, namely TEMs with multiplicative coupling, which are described by the following system of equations (Lazar, 2006a)

$$
\frac{d \boldsymbol{y}}{d t}=(b+u(t)) \boldsymbol{f}(\boldsymbol{y})
$$

where $\boldsymbol{y}=\left[y_{1}, \ldots, y_{n}\right]^{T}, f: \mathbb{R}^{n} \rightarrow \mathbb{R}^{n}$ is an arbitrary continuous non-linear function, and $u \leq c<b$. The solution satisfies $y(t)=x\left(b t+\int_{0}^{t} u(\tau) d \tau\right)$, where $x=\left[x_{1}, \ldots, x_{n}\right]^{T}$ and

$$
\frac{d x}{d t}=f(x) .
$$

The output of this TEM is defined as the sequence of zeros of function $y_{1}$, denoted

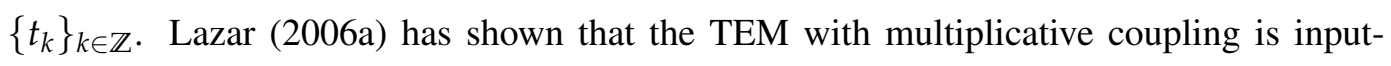
output equivalent with an ideal IF neuron with variable threshold sequence $\left\{\delta_{k+1}-\delta_{k}\right\}_{k \in \mathbb{Z}}$, where $\left\{\delta_{k}\right\}_{k \in \mathbb{Z}}$ denotes the sequence of zeros of function $x_{1}$.

\subsubsection{The asynchronous sigma-delta modulator}

The TEM has also been used as a model for non biological circuits. The ASDM, depicted in Figure 2.3, represents an efficient replacement for the classical A/D converter (Lazar and Tóth, 2004a,b). It consists of an adder, an ideal integrator, and a noninverting Schmitt trigger with parameters $\{\delta / 2,1\}$.

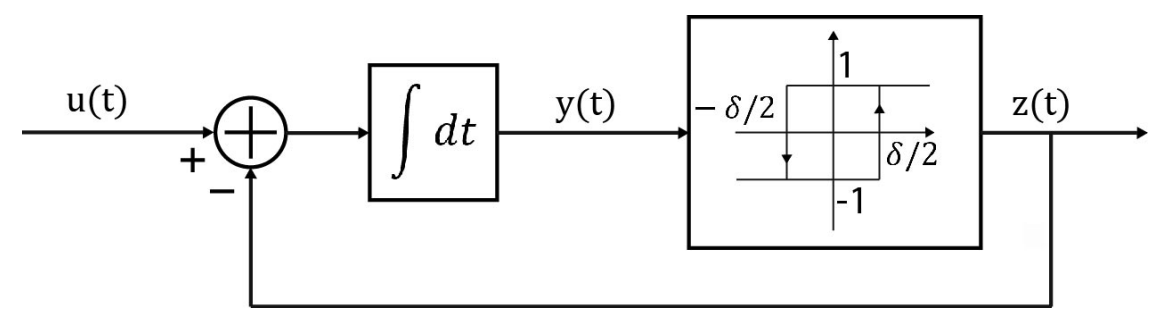

Figure 2.3: The ASDM circuit. 
The input of the circuit is assumed to be bounded by $|u(t)| \leq c<1, \forall t \in \mathbb{R}$. Function $z$ switches between -1 and 1 at times $\mathcal{T}_{u}=\left\{t_{k}\right\}_{k \in \mathbb{Z}}$, with initial value $z\left(t_{0}\right)=-1$. Function $y$ is increasing or decreasing when $z$ is negative or positive, respectively.

Definition 2.12. The ASDM circuit generates output sequence $\mathcal{T}_{u}=\left\{t_{k}\right\}_{k \in \mathbb{Z}}$ when presented with input $u$, satisfying $|u(t)| \leq c<1, \forall t \in \mathbb{R}$, such that

$$
\int_{t_{k}}^{t_{k+1}} u(\tau) d \tau=(-1)^{k}\left[\delta-\left(t_{k+1}-t_{k}\right)\right], \forall k \in \mathbb{Z} .
$$

Lazar and Tóth (2004b) have proposed the following theorem for the reconstruction of $u$ with arbitrary accuracy.

Theorem 2.6. Let $\mathcal{T}_{u}=\left\{t_{k}\right\}_{k \in \mathbb{Z}}$ be the time sequence generated by an ASDM when presented with bounded input $u \in P W_{\Omega},|u(t)| \leq c<1, \forall t \in \mathbb{R}$. Let $\left\{u_{l}\right\}_{l \in \mathbb{Z}}$ be a sequence of bandlimited functions satisfying the recursive equation

$$
u_{l+1}=u_{l}+\mathcal{Z}_{\Omega}\left(u-u_{l}\right), \forall l \in \mathbb{N},
$$

where $u_{0}=\mathcal{Z}_{\Omega} u$ and

$$
\mathcal{Z}_{\Omega}: P W_{\Omega} \rightarrow P W_{\Omega}, \mathcal{Z}_{\Omega} u \triangleq \sum_{k \in \mathbb{Z}} \mathcal{L}_{k}^{\mathcal{T}_{u}} u \cdot g\left(\cdot-s_{k+1}\right)
$$

If $r \triangleq \frac{\Omega}{\pi} \cdot \frac{\delta}{1-c}<1$, then input $u$ can be recovered with arbitrary precision from the associated sequence $\mathcal{T}_{u}$ as

$$
u=\lim _{l \rightarrow \infty} u_{l}
$$

Moreover,

$$
\left\|u-u_{l}\right\|_{L^{2}} \leq r^{l+1} \cdot\|u\|_{L^{2}}, \forall l \in \mathbb{N} \text {. }
$$

Lazar and Tóth (2004b) have presented the following theorem to demonstrate the similarity between Theorem 2.6 and nonuniform sampling theory.

Theorem 2.7. Let $\mathcal{T}_{u}=\left\{t_{k}\right\}_{k \in \mathbb{Z}}$ be the time sequence generated by an ASDM when presented with bounded input $u \in P W_{\Omega},|u(t)| \leq c<1, \forall t \in \mathbb{R}$. Let $\left\{u_{l}\right\}_{l \in \mathbb{Z}}$ be a sequence of bandlimited functions satisfying the recursive equation

$$
u_{l+1}=u_{l}+\mathcal{Z}_{\Omega}^{*}\left(u-u_{l}\right), \forall l \in \mathbb{N},
$$

where $u_{0}=\mathcal{Z}_{\Omega}^{*} u$ and

$$
\mathcal{Z}_{\Omega}^{*}: P W_{\Omega} \rightarrow P W_{\Omega}, \mathcal{Z}_{\Omega}^{*} u=\sum_{k \in \mathbb{Z}} u\left(s_{k+1}\right) \phi_{k}^{\mathcal{T}_{u}}, \forall u \in P W_{\Omega} .
$$




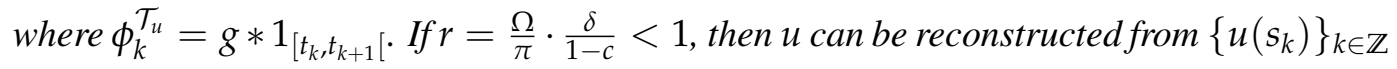
as

$$
u=\lim _{l \rightarrow \infty} u_{l}
$$

Moreover,

$$
\left\|u-u_{l}\right\|_{L^{2}} \leq r^{l+1} \cdot\|u\|_{L^{2}}, \forall l \in \mathbb{N}
$$

The algorithm in Theorem 2.6 has also been presented in matrix form (Lazar and Tóth, 2004b), namely $u=\lim _{l \rightarrow \infty} u_{l}$, where

$$
u_{l}=\boldsymbol{g}^{T} \boldsymbol{P}_{l} \boldsymbol{q}
$$

where $\boldsymbol{g}^{T}$ denotes the transpose of vector $\boldsymbol{g},[\boldsymbol{g}]_{k}=g_{\Omega}\left(\cdot-s_{k}\right), s_{k}=\frac{t_{k-1}+t_{k}}{2}, \forall k \in \mathbb{Z}, \boldsymbol{P}_{l}=$ $\sum_{j=0}^{l}(\boldsymbol{I}-\boldsymbol{G})^{j},[\boldsymbol{G}]_{k, l}=\int_{t_{k}}^{t_{k+1}} g_{\Omega}\left(\tau-s_{l}\right) d \tau$ and $[\boldsymbol{q}]_{k}=(-1)^{k}\left[\delta-\left(t_{k+1}-t_{k}\right)\right], \forall k, l \in \mathbb{Z}$. Moreover, the paper proves that

$$
u=\lim _{l \rightarrow \infty} u_{l}=\lim _{l \rightarrow \infty} \boldsymbol{g}^{T} \boldsymbol{P}_{l} \boldsymbol{q}=\boldsymbol{g}^{T} \boldsymbol{G}^{+} \boldsymbol{q}
$$

Reconstruction formula (2.19) can also be derived using the theory of frames. Sequence $\mathcal{T}_{u}$ satisfies (Lazar and Tóth, 2004b)

$$
\frac{\delta}{1+c} \leq t_{k+1}-t_{k} \leq \frac{\delta}{1-c}, \forall k \in \mathbb{Z}
$$

Then the following holds

$$
\frac{\delta}{1+c} \leq s_{k+1}-s_{k}=\frac{t_{k+1}-t_{k-1}}{2} \leq \frac{\delta}{1-c}, \forall k \in \mathbb{Z}
$$

Therefore, $\left\{s_{k}\right\}_{k \in \mathbb{Z}}$ is relatively separated and $\frac{\delta}{1-c}$ - dense. According to Theorem 2.3 it follows that, if $\frac{\delta}{1-c}<\frac{\pi}{\Omega}$, then $\left\{g_{\Omega}\left(\cdot-s_{k}\right)\right\}_{k \in \mathbb{Z}}$ is a frame on $P W_{\Omega}$ and thus equation (2.19) holds true.

From a computational point of view, the main disadvantage of the reconstruction approach in equations (2.7) and (2.19) is that a new set of functions $\left\{g_{\Omega}\left(\cdot-s_{k}\right)\right\}_{k=2, \ldots, N}$, matrix $G$, and its pseudoinverse $G^{+}$have to be calculated for every sequence $\mathcal{T}_{u}=\left\{t_{k}\right\}_{k=1, \ldots, N}$ of spike times. Alternatively, functions $\left\{g_{\Omega}\left(\cdot-s_{k}\right)\right\}_{k=2, \ldots, N}$ and sequence $\left\{\mathcal{L}_{k}^{\mathcal{T}_{u}} u_{l}\right\}_{l<L}$ are calculated with Theorem 2.6 for every sequence $\mathcal{T}_{u}$ with $L \in \mathbb{N}$ an arbitrarily large number. 


\subsection{Time encoding and decoding in shift-invariant spaces}

The space $P W_{\Omega}$ is spanned by a basis $\{\operatorname{sinc}(\Omega(\cdot-k \pi / \Omega))\}_{k \in \mathbb{Z}}$ of functions that have infinite time support and slow decay, which often creates complexity issues during numerical implementations (Aldroubi and Gröchenig, 2001). The more general SIS is spanned by

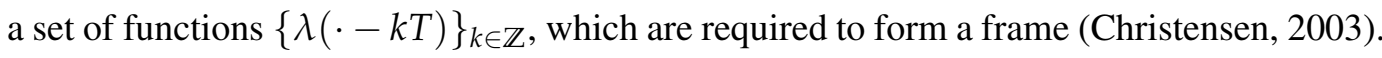

Gontier and Vetterli (2014) extended the results of Lazar and Tóth (2003) to SIS using the non-uniform sampling framework developed by Aldroubi and Feichtinger (1998), Aldroubi and Gröchenig (2001), Feichtinger et al. (1995), Gröchenig (1992, 1993), and Gröchenig and Schwab (2003). The TEMs considered by Gontier and Vetterli (2014) are the crossing TEM (C-TEM) and the integrate-and-fire TEM (IF-TEM), which is a generalization of the ideal IF neuron. The paper designed two algorithms that reconstruct the inputs of the C-TEM and IF-TEM belonging to a SIS and proved the close relationship between the two.

This section introduces the theory of SIS and presents the reconstruction algorithms for the IF-TEM and C-TEM.

The shift invariant space of order 2 generated by function $\lambda$ is defined by (Unser, 2000)

$$
V_{T}^{2}(\lambda)=\left\{u(t)=\sum_{k \in \mathbb{Z}} c_{k} \lambda(t-k T),\left(c_{k}\right)_{k \in \mathbb{Z}} \in l^{2}(\mathbb{R})\right\} .
$$

If $\lambda(t)=\sin (\Omega t) /(\Omega t)$ and $T=\frac{\pi}{\Omega}$ then $u$ is bandlimited and $V_{T}^{2}(\lambda)=P W_{\Omega}$.

An important problem in the theory of SIS is establishing conditions for which the set of functions $\{\lambda(t-k T)\}_{k \in \mathbb{Z}}$ forms a frame on $V_{T}^{2}(\lambda)$. To address this problem, the following periodic function is defined

$$
G_{\lambda}^{T}(\omega) \triangleq\left(\sum_{k \in \mathbb{Z}}\left|\widehat{\lambda}\left(\frac{\omega+2 k \pi}{T}\right)\right|^{2}\right)^{1 / 2}, \forall \omega \in[0,2 \pi] .
$$

The following theorem, presented in (Christensen, 2003), proves several important properties of function $G_{\lambda}^{T}$.

Theorem 2.8. Let $\lambda \in L^{2}(\mathbb{R})$. Then $\{\lambda(\cdot-k T)\}_{k \in \mathbb{Z}}$ is a frame sequence for $V_{T}^{2}(\lambda)$ with bounds $A, B>0$ if and only if

$$
A \leq \frac{G_{\lambda}^{T}(\omega)}{T} \leq B, \text { a.e. } \omega \in[0,2 \pi] \backslash N_{0},
$$

where $N_{0}=\left\{\omega \in[0,2 \pi]: G_{\lambda}^{T}(\omega)=0\right\}$. Moreover, $\left\{\lambda^{\prime}(\cdot-k T)\right\}_{k \in \mathbb{Z}}$ is a Bessel 
sequence for $V_{T}^{2}(\lambda)$ with bound $B^{\prime}>0$ if and only if

$$
\frac{G_{\lambda^{\prime}}^{T}(\omega)}{T} \leq B^{\prime}, \text { a.e. } \omega \in[0,2 \pi]
$$

Gontier and Vetterli (2014) consider shift invariant spaces with integer shifts $V^{2}(\lambda)=$ $V_{1}^{2}(\lambda)$, for which the inner product is defined as

$$
\langle f, g\rangle_{V_{1}^{2}}=\frac{1}{2 \pi} \int_{0}^{2 \pi} \widehat{c}(\omega) \widehat{d}(\omega)^{*} G_{\lambda}(\omega)^{2} d \omega
$$

where $G_{\lambda}=G_{\lambda}^{1}, \widehat{c}(\omega)=\sum_{k \in \mathbb{Z}} c_{k} e^{-i k \omega}, \widehat{d}(\omega)=\sum_{k \in \mathbb{Z}} d_{k} e^{-i k \omega}$, and $c_{k}, d_{k}$ are the coefficients in $V^{2}(\lambda)$ of $f$ and $g$, respectively. The dual frame of $\{\lambda(\cdot-k)\}_{k \in \mathbb{Z}}$, denoted

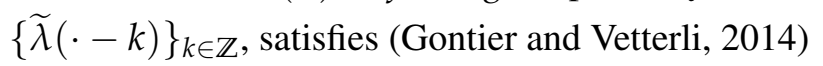

$$
\widetilde{\lambda}(t)=\mathcal{F}^{-1}\left(\frac{\widehat{\lambda}(\omega)}{\left(G_{\lambda}(\omega)\right)^{2}}\right), \forall \omega \in \mathbb{R} .
$$

The dual frame is useful for calculating the coefficients corresponding to the expansion of function $u$ in space $V^{2}(\lambda)$. More generally, it can be used to calculate the coefficients of the orthogonal projection $\mathcal{P}_{V^{2}}$ of an arbitrary function $f \in L^{2}(\mathbb{R})$ onto $V^{2}(\lambda)$ as $\langle f, \tilde{\lambda}(\cdot-$ $k)\rangle_{V^{2}}$, where

$$
\mathcal{P}_{V^{2}} f=\sum_{k \in \mathbb{Z}}\langle f, \tilde{\lambda}(\cdot-k)\rangle_{V^{2}} \cdot \lambda(\cdot-k)
$$

Gontier and Vetterli (2014) restrict function $\lambda$ to space $H^{1}(\mathbb{R})$, i.e., a Sobolev space defined by

$$
H^{1}(\mathbb{R}) \triangleq\left\{f \in L^{2}(\mathbb{R}):\|f\|_{H^{1}}<\infty\right\},\|f\|_{H^{1}}^{2}=\|f\|_{L^{2}}^{2}+\left\|f^{\prime}\right\|_{L^{2}}^{2}
$$

where $f^{\prime}$ is a weak derivative of $f$, which satisfies

$$
\int_{-\infty}^{\infty} f^{\prime}(\tau) v(\tau) d \tau=-\int_{-\infty}^{\infty} f(\tau) v^{\prime}(\tau) d \tau, \forall v \in C_{0}^{\infty}(\mathbb{R})
$$

where $C_{0}^{\infty}(\mathbb{R})$ is the class of smooth functions on $\mathbb{R}$ with compact support.

Let $u \in V_{T}^{2}(\lambda), \lambda \in H^{1}(\mathbb{R})$. Then its weak derivative $u^{\prime}$ is bounded by (Gontier and Vetterli, 2014)

$$
\left\|u^{\prime}\right\|_{L^{2}} \leq \frac{1}{\rho}\|u\|_{L^{2}}
$$

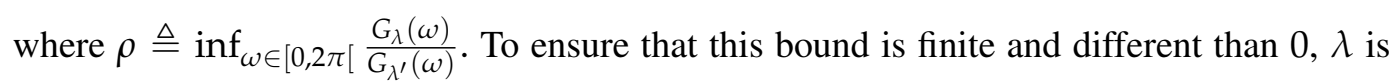


restricted to satisfy conditions (2.21) and (2.22) for $\forall \omega \in[0,2 \pi]$, namely $\lambda \in W$, where

$$
W=\left\{\lambda \in H^{1}(\mathbb{R}): \exists A, B, B^{\prime}>0, A \leq G_{\lambda}(\omega) \leq B, G_{\lambda^{\prime}}(\omega) \leq B^{\prime}, \forall \omega \in[0,2 \pi]\right\}
$$

Function $\lambda$ is required to belong to Sobolev space $H_{1}(\mathbb{R})$ such that the assumptions are satisfied in the next lemma, which establishes two properties for space $V^{2}(\lambda)$ (Gontier and Vetterli, 2014).

Lemma 2.3. Let $\lambda \in W$. Then $V^{2}(\lambda)$ is a RKHS and $V^{2}(\lambda) \hookrightarrow C(\mathbb{R})$, where $C(\mathbb{R})$ denotes the class of continuous functions on $\mathbb{R}$.

The reproducing kernel on $V^{2}(\lambda)$ is denoted by $K: \mathbb{R}^{2} \rightarrow \mathbb{R}$ and has the expression (Gontier and Vetterli, 2014)

$$
K(x, t)=\sum_{k \in \mathbb{Z}} \lambda(x-k) \widetilde{\lambda}(t-k)=\sum_{k \in \mathbb{Z}} \tilde{\lambda}(x-k) \lambda(t-k) .
$$

Definition 2.13. A crossing TEM (C-TEM) with continuous test functions $\left\{\Phi_{k}\right\}$ generates a sequence $\mathcal{C} \mathcal{T}_{u}=\left\{t_{k}\right\}_{k \in \mathbb{Z}}$, when presented with input $u \in V^{2}(\lambda)$, such that

A) The value of $\Phi_{k}$ at a given time is $\Phi_{k}(t)=\Phi\left(t,\left\{t_{j}, j \leq k\right\}\right)$, where $\Phi: \mathbb{R} \times\left\{t_{j}, j \leq\right.$ $k\} \rightarrow \mathbb{R}$.

B) $u\left(t_{k+1}\right)=\Phi_{k}\left(t_{k+1}\right)$;

C) $\left.u(t) \neq \Phi_{k}(t), \forall t \in\right] t_{k}, t_{k+1}[$.

The problem of reconstructing $u$ from the output of a C-TEM is the same as the one of reconstructing $u$ from its nonuniform samples at times $\mathcal{C} \mathcal{T}_{u}$. The following operator is required to design a reconstruction algorithm.

$$
\mathcal{V}: V^{2}(\lambda) \rightarrow V^{2}(\lambda), \mathcal{V} u \triangleq \sum_{k \in \mathbb{Z}} u\left(t_{k}\right) 1_{\left[s_{k}, s_{k+1}[\right.}
$$

where $s_{k}=\frac{t_{k-1}+t_{k}}{2}, \forall k \in \mathbb{Z}$.

The following theorem was proven in (Gontier and Vetterli, 2014) and generalizes Theorem 2.4 proven by Feichtinger and Gröchenig (1994) for bandlimited spaces.

Theorem 2.9. Let $\mathcal{C} \mathcal{T}_{u}=\left\{t_{k}\right\}_{k \in \mathbb{Z}}$ be the sequence generated by a $C$-TEM when presented with input $u \in V^{2}(\lambda), \lambda \in W$. Then $u$ can be reconstructed iteratively from $\mathcal{C} \mathcal{T}_{u}$ provided that there exists $\Delta>0$ such that $\mathcal{C} \mathcal{T}_{u}$ is $\Delta$-dense and

$$
\Delta<\pi \rho .
$$


where $\rho=\inf _{\omega \in[0,2 \pi[} \frac{G_{\lambda}(\omega)}{G_{\lambda^{\prime}}(\omega)}$. The reconstruction is performed with

$$
\begin{gathered}
u_{1}=\mathcal{P}_{V^{2}} \mathcal{V} u \\
u_{n+1}=u_{1}+\left(\mathcal{I}-\mathcal{P}_{V^{2}} \mathcal{V}\right) u_{n}
\end{gathered}
$$

where $\mathcal{I}$ is the identity operator. The functions $u_{n}$ satisfy

$$
\left\|u-u_{n}\right\|_{L^{2}} \leq\left(\frac{\Delta}{\pi \rho}\right)^{n}\|u\|_{L^{2}}, \forall n \in \mathbb{N}^{*}
$$

For the particular case $V^{2}(\lambda)=P W_{\Omega}, \rho=\frac{1}{\Omega}$ and the requirement (4.6) is $\Delta<\frac{\pi}{\Omega}$, which is in line with the result for bandlimited spaces in Theorem 2.4.

Definition 2.14. An IF-TEM with test functions $\left\{\Phi_{k}\right\}$ generates a sampling sequence $\mathcal{I}_{u}=$

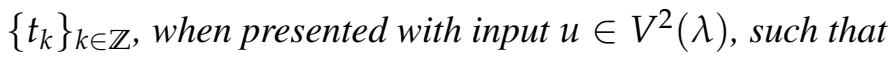

A) The value of $\Phi_{k}$ at a given time is $\Phi_{k}(t)=\Phi\left(t,\left\{t_{j}, j \leq k\right\}\right)$, where $\Phi: \mathbb{R} \times\left\{t_{j}, j \leq\right.$ $k\} \rightarrow \mathbb{R}$.

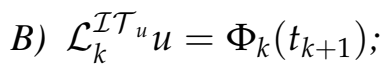

C) $\left.\int_{t_{k}}^{t} u(\tau) d \tau \neq \Phi_{k}(t), \forall t \in\right] t_{k}, t_{k+1}[$,

where $\mathcal{L}_{k}^{\mathcal{I} \mathcal{T}_{u}}$ is an operator mapping function $u$ onto the real axis, i.e., $\mathcal{L}_{k}^{\mathcal{I} \mathcal{T}_{u}}$ : $V^{2}(\lambda) \rightarrow \mathbb{R}, \mathcal{L}_{k}^{\mathcal{I} \mathcal{T}_{u}} u \triangleq \int_{t_{k}}^{t_{k+1}} u(t) d t$

The ideal IF neuron is an IF-TEM with test functions $\Phi_{k}(t)=\bar{\delta}-b\left(t-t_{k}\right), \forall k \in \mathbb{Z}$, where $\bar{\delta} \triangleq C \delta$, and $\delta, C$, and $b$ are the threshold, integration constant, and bias, respectively. Moreover, the ASDM circuit can be modelled as an IF-TEM with test functions $\Phi_{k}(t)=$ $(-1)^{k}\left[\delta-\left(t-t_{k}\right)\right], \forall k \in \mathbb{Z}$.

The following operator is used to reconstruct the input of an IF-TEM

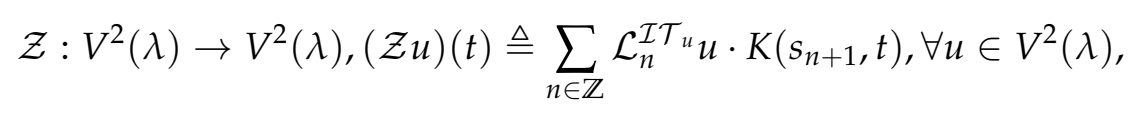

where $K$ is the reproducing kernel in $V^{2}(\lambda)$. To show the relationship between the IF-TEM and the C-TEM, operator $V^{\prime}$ is defined as

$$
\mathcal{V}^{\prime}: V^{2}(\lambda) \rightarrow V^{2}(\lambda), \mathcal{V}^{\prime} u \triangleq \sum_{k \in \mathbb{Z}} u\left(s_{k+1}\right) 1_{\left[t_{k}, t_{k+1}\right.}[.
$$

Gontier and Vetterli (2014) have proven that operator $\mathcal{P}_{V^{2}} \mathcal{V}^{\prime}$ is the adjoint of $\mathcal{P}_{V^{2}} \mathcal{Z}$, i.e.

$$
\left\langle f, \mathcal{P}_{V^{2}} \mathcal{V}^{\prime} g\right\rangle_{V^{2}}=\left\langle\mathcal{P}_{V^{2}} \mathcal{Z} f, g\right\rangle_{V^{2}}, \forall f, g \in V^{2}(\lambda)
$$


The operators $\mathcal{V}$ and $\mathcal{V}^{\prime}$ are very similar, and due to (2.28) the IF-TEM is called the quasi-adjoint of the C-TEM (Gontier and Vetterli, 2014).

Theorem 2.10. Let $\mathcal{I} \mathcal{T}_{u}=\left\{t_{k}\right\}_{k \in \mathbb{Z}}$ be the sequence generated by an IF-TEM when presented with input $u \in V^{2}(\lambda), \lambda \in W$. Then $u$ can be reconstructed iteratively from $\mathcal{I} \mathcal{T}_{u}$ provided that there exists $\Delta>0$ such that $\mathcal{I T}_{u}$ is $\Delta$-dense and

$$
\Delta<\pi \rho .
$$

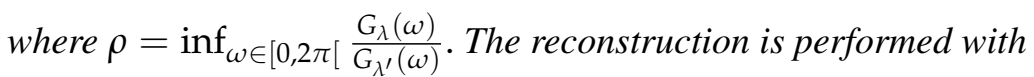

$$
\begin{gathered}
u_{1}=\mathcal{P}_{V^{2}} \mathcal{Z} u \\
u_{n+1}=u_{1}+\left(\mathcal{I}-\mathcal{P}_{V^{2}} \mathcal{Z}\right) u_{n}
\end{gathered}
$$

where $\mathcal{I}$ is the identity operator. The functions $u_{n}$ satisfy

$$
\left\|u-u_{n}\right\|_{L^{2}} \leq\left(\frac{\Delta}{\pi \rho}\right)^{n}\|u\|_{L^{2}}, \forall n \in \mathbb{N}^{*} .
$$

Theorem 2.6 is the particular case of Theorem 2.10 for $u \in P W_{\Omega}$, where $\rho=\frac{1}{\Omega}$ and the requirement (4.7) is $\Delta<\frac{\pi}{\Omega}$.

From a computational point of view, the main disadvantage of the reconstruction approach in Theorem 2.10 is that functions $\left\{K\left(s_{k}, \cdot\right)\right\}_{k=1, \ldots, N}$ and values $\left\{\mathcal{L}_{k}^{\mathcal{I} \mathcal{T}_{u}} u_{l}\right\}_{l<L}$ are calculated for every sequence $\mathcal{I} \mathcal{T}_{u}$, where $L \in \mathbb{N}$ is an arbitrarily large number.

\subsection{Conclusions}

This chapter presented two dual sampling methods, namely nonuniform sampling and sampling based on timing. As a consequence of this duality, the corresponding two algorithms for reconstructing a function from its samples are studied in a unifying manner.

An important class of models that performs sampling based on timing is the TEM. Several models of the biological spiking neuron have been represented as TEMs, including the ideal IF model, the LIF neuron, the IF model with variable threshold and the IF model with absolute refractory period. The TEM has also been proposed as a model for the ASDM, an efficient encoding circuit representing a suitable replacement for the classical A/D converter.

The existent algorithms for reconstructing the input of a TEM belonging to bandlimited or shift-invariant spaces exploit the classical formulation of time encoding, where the stimulus is projected onto a set of input dependent frame functions. As a consequence, input $u^{j}$ 
is reconstructed from sequence $\mathcal{T}_{u^{j}}$ in a space spanned by a new set of functions for every $j$. This process becomes computationally demanding for a large number of reconstructions. 


\section{Chapter 3}

\section{A novel framework for reconstructing bandlimited signals encoded by integrate-and-fire}

\section{neurons}

The integrate-and-fire (IF) neuron, one of the most common models for describing the behaviour of spiking neurons (Lapicque, 1907, Tuckwell, 1988), belongs to the larger class of models called time encoding machines (TEMs) (Lazar and Tóth, 2003). Practical algorithms have been proposed and evaluated for reconstructing bandlimited inputs $u$ from the output sequences $\mathcal{T}_{u}$ of TEMs (Lazar, 2004, Lazar and Pnevmatikakis, 2008a, Lazar and Tóth, 2004a,b).

The existing reconstruction algorithms for signals encoded with IF neurons are studied in an unifying manner using nonuniform sampling theory (Feichtinger and Gröchenig, 1994). Specifically, the encoding of a signal $u$ with an IF neuron is formulated as the projection of $u$ onto a set of functions $\left\{\phi_{k}^{\mathcal{T}_{u}}\right\}_{k \in \mathbb{Z}}$, which depend on the nonuniform sequence of time points $\mathcal{T}_{u}$. A disadvantage of this formulation is that the sequence $\left\{\phi_{k}^{\mathcal{T}_{u}}\right\}_{k \in \mathbb{Z}}$ is input dependent and, as a consequence, every input $u$ is reconstructed in a space spanned by a new set of frame functions. Moreover, the reconstruction algorithms are based on solving a linear system whose coefficients are different for every input (Lazar and Pnevmatikakis, 2008a). In essence, reconstructing several inputs $u^{j}$ from sequences $\mathcal{T}_{u^{j}}=\left\{t_{k}^{j}\right\}_{k=1, N+1}$ involves recalculating the frame functions and solving a new linear system for every sequence $j$. For large values of $N$ this process becomes computationally demanding.

This chapter presents new theoretical results that form the basis for a new method of reconstructing a input function from its projections onto set of functions $\left\{\phi_{k}\right\}_{k \in \mathbb{Z}}$ that, unlike the state-of-the art method, are not dependent on the input. This method is a required 
tool for developing new efficient algorithms for reconstructing a function $u$ from the output sequence $\mathcal{T}_{u}$ of an IF neuron.

A new framework is introduced for studying time encoding and decoding by reformulating the input dependent nonuniform sampling operation performed by an IF neuron on a function $u$ as an equivalent input independent uniform sampling operation performed on an auxiliary function $\bar{\psi}^{\prime}$. This framework is particularly useful because it enables to apply the system modelling and analysis tools already available for uniformly sampled systems.

This framework forms the basis for two new reconstruction algorithms that are significantly faster than the ones proposed by Lazar (2004) and Lazar and Pnevmatikakis (2008a), respectively. The trade-off for the computational speed is reconstructing a function $\bar{\psi}^{\prime}$ that is not strictly bandlimited. Numerical simulations are used to show the performance of the new algorithms.

The chapter is structured as follows. Section 3.1 presents the new method for reconstructing a function from local averages. Section 3.2 presents detailed algorithms corresponding to the standard state-of-the-art reconstruction methods for IF neurons. Section 3.3 introduces the new theoretical framework for describing the sampling operation performed by an ideal IF neuron. Two novel fast reconstruction algorithms for bandlimited signals are introduced in Section 3.4. Section 3.5 presents numerical simulation results that demonstrate the performance of the new algorithms in comparison with the standard state-of-the-art methods. Conclusions are in Section 3.6.

\subsection{A new method of reconstructing functions from local aver- ages}

Let $P W_{\Omega}$ denote the Paley-Wiener space of bandwidth $\Omega$, defined in (2.1). Let $\mathcal{Z}_{\Omega}, \mathcal{Z}_{\Omega}{ }^{\prime}$ : $P W_{\Omega} \rightarrow P W_{\Omega}$ be two operators defined as

$$
\begin{gathered}
\mathcal{Z}_{\Omega} u \triangleq \sum_{k \in \mathbb{Z}} \mathcal{L}_{k}^{\mathcal{T}} u \cdot g_{\Omega}\left(\cdot-s_{k+1}\right), \\
\mathcal{Z}_{\Omega}^{\prime} u \triangleq \sum_{k \in \mathbb{Z}} \mathcal{L}_{k}^{\left\{s_{k}\right\}_{k \in \mathbb{Z}} u \cdot g_{\Omega}\left(\cdot-t_{k}\right),}
\end{gathered}
$$

where $g_{\Omega}=\frac{\sin (\Omega \cdot)}{\pi \cdot}, \mathcal{T}=\left\{t_{k}\right\}_{k \in \mathbb{Z}}, s_{k+1}=\frac{t_{k}+t_{k+1}}{2}$, and $\mathcal{L}_{k}^{\mathcal{T}} u=\int_{t_{k}}^{t_{k+1}} u(\tau) d \tau, \forall k \in \mathbb{Z}$. Feichtinger and Gröchenig (1994) have proposed an iterative reconstruction algorithm for $u$ using operator $\mathcal{Z}_{\Omega}{ }^{\prime}$.

The following lemma proves two important properties of operator $\mathcal{Z}_{\Omega}$. The concepts of $\Delta-$ dense and relatively separated sequence used in this lemma were introduced in definitions 2.2 and 2.4 .

Lemma 3.1. Let $\mathcal{T}=\left\{t_{k}\right\}_{k \in \mathbb{Z}}$ be a $\Delta$-dense, relatively separated and strictly increasing 
sequence. Then $\mathcal{Z}_{\Omega}$ is a well defined operator provided that $\Delta<\frac{\pi}{\Omega}$.

Proof. Sequence $\mathcal{T}$ is relatively separated if

$$
\exists \zeta>0,\left|t_{k}-t_{p}\right|>\zeta, \forall k, p \in \mathbb{Z}
$$

Then the following holds.

$$
\begin{aligned}
\left|t_{k}-t_{p}\right|>\zeta & \Leftrightarrow\left|t_{k+1}-t_{k}\right|>\zeta \Rightarrow\left|t_{k+1}-t_{k-1}\right|>2 \zeta \\
& \Leftrightarrow\left|\frac{t_{k}+t_{k+1}}{2}-\frac{t_{k-1}+t_{k}}{2}\right|=\left|s_{k+1}-s_{k}\right|>\zeta \\
& \Leftrightarrow\left|s_{k}-s_{p}\right|>\zeta, \forall k, p \in \mathbb{Z},
\end{aligned}
$$

Moreover,

$$
\left|s_{k+1}-s_{k}\right|=\left|t_{k}-s_{k}\right|+\left|s_{k+1}-t_{k}\right|=\left|\frac{t_{k}-t_{k-1}}{2}\right|+\left|\frac{t_{k+1}-t_{k}}{2}\right|<2 \frac{\Delta}{2}=\Delta, \forall k \in \mathbb{Z} .
$$

Therefore, the sequence $\left\{s_{k}\right\}_{k \in \mathbb{Z}}$ is relatively separated and $\Delta$ - dense, and, according to Theorem 2.3, $\left\{g_{\Omega}\left(\cdot-s_{k}\right)\right\}_{k \in \mathbb{Z}}$ is a frame for $P W_{\Omega}$ provided that $\Delta<\frac{\pi}{\Omega}$. The synthesis operator of this frame $\mathcal{C}: l^{2}(\mathbb{R}) \rightarrow P W_{\Omega}$ is defined by

$$
\mathcal{C} d=\sum_{k \in \mathbb{Z}} d_{k} \cdot g_{\Omega}\left(\cdot-s_{k+1}\right), \forall d \in l^{2}(\mathbb{R}) .
$$

Let $\mathcal{L}^{\mathcal{T}}: P W_{\Omega} \rightarrow l^{2}(\mathbb{R})$ be an operator defined by $\mathcal{L}^{\mathcal{T}} u \triangleq\left\{\mathcal{L}_{k}^{\mathcal{T}} u\right\}_{k \in \mathbb{Z}}, \forall u \in P W_{\Omega}$. Then $\mathcal{Z}_{\Omega}=\mathcal{C} \circ \mathcal{L}^{\mathcal{T}}$ is well defined provided that $\mathcal{L}^{\mathcal{T}}$ is well defined, which will be proven as follows.

Any function $u \in P W_{\Omega}$ is continuous and, according to the mean value theorem for integrals, it follows that for all $k \in \mathbb{Z}$, there exist $\xi_{k}, \chi_{k} \in\left[t_{k}, t_{k+1}\right]$ such that

$$
\begin{aligned}
& \mathcal{L}_{k}^{\mathcal{T}} u=\left(t_{k+1}-t_{k}\right) u\left(\xi_{k}\right), \\
& \mathcal{L}_{k}^{\mathcal{T}} u^{2}=\left(t_{k+1}-t_{k}\right) u^{2}\left(\chi_{k}\right) .
\end{aligned}
$$


Using the Cauchy-Schwarz and AM-GM inequalities, it follows that

$$
\begin{aligned}
\sum_{k \in \mathbb{Z}}\left(\mathcal{L}_{k}^{\mathcal{T}} u\right)^{2} & =\left|\sum_{k \in \mathbb{Z}}\left(t_{k+1}-t_{k}\right)\left[\left(t_{k+1}-t_{k}\right) u^{2}\left(\chi_{k}\right)+\left(t_{k+1}-t_{k}\right)\left(u^{2}\left(\xi_{k}\right)-u^{2}\left(\chi_{k}\right)\right)\right]\right| \\
& \leq \Delta\left[\sum_{k \in \mathbb{Z}} \mathcal{L}_{k}^{\mathcal{T}} u^{2}+\Delta \sum_{k \in \mathbb{Z}}\left|\int_{\chi_{k}}^{\xi_{k}} 2 u(\tau) u^{\prime}(\tau) d \tau\right|\right] \\
& \leq \Delta\left[\|u\|_{L^{2}}^{2}+2 \Delta \sum_{k \in \mathbb{Z}}\left|\int_{\chi_{k}}^{\xi_{k}} u^{2}(\tau) d \tau\right|^{1 / 2} \cdot\left|\int_{\chi_{k}}^{\xi_{k}} u^{\prime 2}(\tau) d \tau\right|^{1 / 2}\right] \\
& \leq \Delta\left[\|u\|_{L^{2}}^{2}+\Delta \sum_{k \in \mathbb{Z}}\left|\int_{\chi_{k}}^{\xi_{k}} u^{2}(\tau) d \tau\right|+\Delta \sum_{k \in \mathbb{Z}}\left|\int_{\chi_{k}}^{\xi_{k}} u^{\prime 2}(\tau) d \tau\right|\right] \\
& \leq \Delta\left[\|u\|_{L^{2}}^{2}+\Delta\|u\|_{L^{2}}^{2}+\Delta\left\|u^{\prime}\right\|_{L^{2}}^{2}\right]
\end{aligned}
$$

For a function $u \in P W_{\Omega}$, the Bernstein inequality states that (Lazar and Tóth, 2004b)

$$
\left\|u^{\prime}\right\|_{L^{2}} \leq \Omega\|u\|_{L^{2}}
$$

Then the following holds (3.1), (3.2)

$$
\left\|\mathcal{L}^{\mathcal{T}} u\right\|_{l^{2}}^{2}=\sum_{k \in \mathbb{Z}}\left(\mathcal{L}_{k}^{\mathcal{T}} u\right)^{2} \leq\|u\|_{L^{2}}^{2}\left(\Delta^{2}+\Delta^{2} \Omega+\Delta\right) .
$$

Function $u \in P W_{\Omega}$ can be reconstructed from local averages with operator $\mathcal{Z}_{\Omega}$ as follows.

Theorem 3.1. Let $\mathcal{T}=\left\{t_{k}\right\}_{k \in \mathbb{Z}}$ be a $\Delta$-dense, relatively separated and strictly increasing sequence, and let $u \in P W_{\Omega}$, such that $\Delta<\frac{\pi}{\Omega}$. Let $\left\{u_{n}\right\}_{n \in \mathbb{Z}}$ be a sequence of functions satisfying

$$
\begin{gathered}
u_{0}=\mathcal{Z}_{\Omega} u, \\
u_{n+1}=u_{n}+\mathcal{Z}_{\Omega}\left(u-u_{n}\right), \forall n \in \mathbb{N} .
\end{gathered}
$$

Then $u$ can be reconstructed from sequence $\left\{\mathcal{L}_{k}^{\mathcal{T}} u\right\}_{k \in \mathbb{Z}}$ as $u=\lim _{n \rightarrow \infty} u_{n}$. Moreover,

$$
\left\|u-u_{n}\right\|_{L^{2}} \leq\left(\frac{\Delta \Omega}{\pi}\right)^{n+1}\|u\|_{L^{2}}
$$

Proof. By induction, the following holds

$$
u_{n}=\sum_{p=0}^{n}\left(\mathcal{I}-\mathcal{Z}_{\Omega}\right)^{p} \mathcal{Z}_{\Omega} u, \forall n \in \mathbb{N},
$$


where $\mathcal{I}$ represents the identity operator. Operator $\mathcal{I}-\mathcal{Z}_{\Omega}$ is bounded by $\frac{\Delta \Omega}{\pi}<1$, due to Lemma 3.1. Therefore $\mathcal{Z}_{\Omega}^{-1}$ can be expanded in Neumann series as

$$
\mathcal{Z}_{\Omega}^{-1}=\sum_{p=0}^{\infty}\left(\mathcal{I}-\mathcal{Z}_{\Omega}\right)^{p}
$$

It follows that $\lim _{n \rightarrow \infty} u_{n}=u$. Moreover,

$$
\begin{aligned}
u-u_{n} & =\sum_{p=n+1}^{\infty}\left(\mathcal{I}-\mathcal{Z}_{\Omega}\right)^{p} \mathcal{Z}_{\Omega} u \\
& =\left(\mathcal{I}-\mathcal{Z}_{\Omega}\right)^{n+1} \sum_{p=0}^{\infty}\left(\mathcal{I}-\mathcal{Z}_{\Omega}\right)^{p} \mathcal{Z}_{\Omega} u \\
& =\left(\mathcal{I}-\mathcal{Z}_{\Omega}\right)^{n+1} u .
\end{aligned}
$$

The proof of inequality (3.4) closely follows the proof of Proposition 1 from (Lazar, 2004), and requires calculating the adjoint of $\mathcal{Z}_{\Omega}$, which satisfies

$$
\left\langle\mathcal{Z}_{\Omega} u, v\right\rangle_{L^{2}}=\left\langle\left(\mathcal{C} \circ \mathcal{L}^{\mathcal{T}}\right) u, v\right\rangle_{L^{2}}=\left\langle u,\left(\mathcal{L}^{\mathcal{T} *} \circ \mathcal{C}^{*}\right) v\right\rangle_{L^{2}}=\left\langle u, \mathcal{Z}_{\Omega}^{*} v\right\rangle_{L^{2}}
$$

for $\forall u, v \in P W_{\Omega}$. According to (Lazar and Pnevmatikakis, 2008a), operator $\mathcal{L}^{\mathcal{T}}$ has the expression

$$
\mathcal{L}^{\mathcal{T}} u=\left\{\int_{t_{k}}^{t_{k+1}} u(\tau) d \tau\right\}_{k \in \mathbb{Z}}=\left\{\left\langle u, \phi_{k}^{\mathcal{T}}\right\rangle\right\}_{k \in \mathbb{Z}}, \forall u \in P W_{\Omega}
$$

where $\phi_{k}^{\mathcal{T}}=g_{\Omega} * 1_{\left[t_{k}, t_{k+1}[\right.}, \forall k \in \mathbb{Z}$, and $1_{\left[t_{k}, t_{k+1}[\right.}$ is the characteristic function of interval $\left[t_{k}, t_{k+1}\left[\right.\right.$. Lazar and Pnevmatikakis (2008a) have proven that $\left\{\phi_{k}^{\mathcal{T}}\right\}_{k \in \mathbb{Z}}$ is a frame for $P W_{\Omega}$ provided that $\mathcal{T}$ is $\Delta-$ dense and $\Delta<\frac{\pi}{\Omega}$. It follows that $\mathcal{L}^{\mathcal{T}}$ is the analysis operator of this frame, and thus its adjoint $\mathcal{L}^{\mathcal{T} *}$ represents the synthesis operator, satisfying

$$
\mathcal{L}^{\mathcal{T} *} d=\sum_{k \in \mathbb{Z}} d_{k} \cdot \phi_{k}^{\mathcal{T}}, \forall d \in l^{2}(\mathbb{R})
$$

Moreover, $\mathcal{C}^{*}$ represents the analysis operator of frame $\left\{g_{\Omega}\left(\cdot-s_{k}\right)\right\}_{k \in \mathbb{Z}}$

$$
\mathcal{C}^{*} u=\left\{\left\langle u, g_{\Omega}\left(\cdot-s_{k+1}\right)\right\rangle\right\}_{k \in \mathbb{Z}}=\left\{u\left(s_{k+1}\right)\right\}_{k \in \mathbb{Z}}, \forall u \in P W_{\Omega} .
$$

Therefore, $\mathcal{Z}_{\Omega}^{*}$ has the expression

$$
\mathcal{Z}_{\Omega}^{*} u=\mathcal{L}^{\mathcal{T} *}\left(\mathcal{C}^{*} u\right)=\sum_{k \in \mathbb{Z}} u\left(s_{k+1}\right) \cdot \phi_{k}^{\mathcal{T}}, \forall u \in P W_{\Omega} .
$$

The following holds due to the properties of the adjoint and Proposition 3 from (Feichtinger 
and Gröchenig, 1994)

$$
\left\|u-\mathcal{Z}_{\Omega} u\right\|_{L^{2}}=\left\|u-\mathcal{Z}_{\Omega}^{*} u\right\|_{L^{2}}<\frac{\Delta \Omega}{\pi}\|u\|_{L^{2}}, \forall u \in P W_{\Omega} .
$$

The required result follows from inequalities (3.5) and (3.7).

The following corollary was proven in (Lazar, 2004) and is given here for consistency.

Corollary 3.1. Under the assumptions of Theorem 3.1, function u can be reconstructed from sequence $\left\{\mathcal{L}_{k}^{\mathcal{T}} u\right\}_{k \in \mathbb{Z}}$ as

$$
u=\lim _{n \rightarrow \infty} u_{n}=\lim _{n \rightarrow \infty} \boldsymbol{g}^{T} \boldsymbol{P}_{n} \boldsymbol{q},
$$

where $[\boldsymbol{g}]_{k}=g_{\Omega}\left(\cdot-s_{k+1}\right), \boldsymbol{P}_{n}=\sum_{p=0}^{n}(\boldsymbol{I}-\boldsymbol{G})^{p}$, I denotes the unit matrix, $[\boldsymbol{G}]_{m, k}=$ $\mathcal{L}_{m}^{\mathcal{T}}\left(g_{\Omega}\left(\cdot-s_{k+1}\right)\right)$, and $[\boldsymbol{q}]_{k}=\mathcal{L}_{k}^{\mathcal{T}} u, \forall m, k \in \mathbb{Z}$. Moreover,

$$
u=g^{T} G^{+} q
$$

Proof. See the proof of Corollary 2 from (Lazar, 2004).

The choice of the Moore-Penrose pseudoinverse in (3.8) determines a set of coefficients $\boldsymbol{G}^{+} \boldsymbol{q}$ with minimal $\ell^{2}$ norm. Due to the properties of frame $\left\{g_{\Omega}\left(\cdot-s_{k+1}\right)\right\}_{k \in \mathbb{Z}}$, the following holds

$$
B^{-1}\|u\|_{L^{2}}^{2} \leq\left\|\boldsymbol{G}^{+} \boldsymbol{q}\right\|_{\ell^{2}}^{2} \leq A^{-1}\|u\|_{L^{2}}^{2}, \forall u \in P W_{\Omega} .
$$

where $A, B$ denote the lower and upper bound, respectively (see Appendix A). In a practical setting, where the reconstructed signal $u$ is transmitted onwards through a communication channel, low energy consumption is usually desired. The choice of the Moore-Penrose pseudoinverse is motivated by the need to minimize $\|u\|_{L^{2}}^{2}$ which, for current or voltage signals, is equivalent to minimizing the transmission energy (Gabor, 1946).

The results introduced in Theorem 3.1 and Corollary 3.1 are used to develop two new reconstruction algorithms in Section 3.4. 


\subsection{Direct reconstruction algorithms for inputs encoded with ideal IF neurons}

Let $\mathcal{T}_{u}=\left\{t_{k}\right\}_{k \in \mathbb{Z}}$ be the output sequence of an ideal IF neuron with parameters $\{b, \bar{\delta}\}$, for a given bounded input $u \in P W_{\Omega},|u| \leq c<b$.

Sequence $\mathcal{T}_{u}$ is relatively separated and $\frac{\bar{\delta}}{b-c}-$ dense (Lazar and Pnevmatikakis, 2008a). Therefore, provided that $\frac{\bar{\delta}}{b-c}<\frac{\pi}{\Omega}$, function $u$ can be reconstructed iteratively (Lazar, 2004) and non-iteratively (Lazar and Pnevmatikakis, 2008a) from sequence $\mathcal{T}_{u}$. These methods represent particular cases of Theorem 3.1 and Corollary 3.1 .

The reconstructed input function $u_{r e c}$ is computed on the uniform grid $\{l \varepsilon\}_{l=0, \ldots, L}$, where $\varepsilon$ is the sampling period used in reconstruction. The reconstruction algorithm for $u_{\text {rec }}$ from the finite sequence of spike times $\mathcal{T}_{u}=\left\{t_{k}\right\}_{k=1, \ldots, N+1}$ can be summarised as follows (Lazar and Pnevmatikakis, 2008a).

The corresponding theoretical results underlying the next two algorithms have been presented in Chapter 2. The algorithms are given in the following for completeness.

Step 1. Calculate $q$

$$
[\boldsymbol{q}]_{m}=\bar{\delta}-b\left(t_{m+1}-t_{m}\right), m=1, \ldots, N
$$

Step 2. Calculate $G, g_{\Omega}\left(l \varepsilon-s_{m+1}\right), l=0, \ldots, L$, where

$$
\begin{gathered}
g_{\Omega}\left(l \varepsilon-s_{m+1}\right)=\frac{\sin \left(\Omega\left(l \varepsilon-s_{m+1}\right)\right)}{\pi\left(l \varepsilon-s_{m+1}\right)}, \\
{[\boldsymbol{G}]_{k m}=\int_{t_{k}}^{t_{k+1}} g_{\Omega}\left(\tau-s_{m+1}\right) d \tau, k, m=1, \ldots, N ; ;}
\end{gathered}
$$

Step 3. Calculate $G^{+}$;

Step 4. Calculate $c$

$$
c=G^{+} q
$$

Step 5. Calculate $u_{r e c}(l \varepsilon), l=0, \ldots, L$, where

$$
u_{r e c}(l \varepsilon)=\sum_{m=1}^{N} c_{m} g_{\Omega}\left(l \varepsilon-s_{m+1}\right)
$$

An iterative algorithm, for which accuracy can be traded off for shorter computation time, may be preferred for the scenario in which the time allocated for reconstruction is limited (Strohmer, 1991). The following algorithm computes $u_{\text {rec }}$ iteratively from sequence $\mathcal{T}_{u}$ (Lazar, 2004). 
Step 1. Calculate $q$

$$
[\boldsymbol{q}]_{m}=\bar{\delta}-b\left(t_{m+1}-t_{m}\right), m=1, \ldots, N
$$

Step 2. Calculate $G, g_{\Omega}\left(l \varepsilon-s_{m+1}\right), l=0, \ldots, L$, where

$$
\begin{gathered}
g_{\Omega}\left(l \varepsilon-s_{m+1}\right)=\frac{\sin \left(\Omega\left(l \varepsilon-s_{m+1}\right)\right)}{\pi\left(l \varepsilon-s_{m+1}\right)}, \\
{[\boldsymbol{G}]_{k m}=\int_{t_{k}}^{t_{k+1}} g_{\Omega}\left(\tau-s_{m+1}\right) d \tau, k, m=1, \ldots, N ; ;}
\end{gathered}
$$

Step 3. Calculate $\boldsymbol{P}_{n}$ iteratively as

$$
\begin{gathered}
\boldsymbol{P}_{0}=\boldsymbol{I} ; \\
\boldsymbol{P}_{j}=\boldsymbol{I}+\boldsymbol{P}_{j-1}(\boldsymbol{I}-\boldsymbol{G}), j=1, \ldots, n ;
\end{gathered}
$$

Step 4. Calculate $c$

$$
c=P_{n} \boldsymbol{q}
$$

Step 5. Calculate $u_{\text {rec }}(l \varepsilon), l=0, \ldots, L$, where

$$
u_{r e c}(l \varepsilon)=\sum_{m=1}^{N} c_{m} g_{\Omega}\left(l \varepsilon-s_{m+1}\right) .
$$

In order to reconstruct a set of inputs $\left\{u^{j}\right\}_{j=1, \ldots, R}$ from the corresponding set of spike sequences $\left\{\mathcal{T}_{u^{j}}\right\}_{j=1, \ldots, R}$ a new set of functions $\left\{g_{\Omega}\left(\cdot-s_{k}\right)\right\}_{k=2, \ldots, N}$, matrix $G$, and sequence $\left\{\boldsymbol{P}_{j}\right\}_{j=0, \ldots, n}$ or pseudoinverse $\boldsymbol{G}^{+}$have to be calculated for every $j$, which is computationally demanding for large values of $R$ and $N$.

\subsection{The integrate-and-fire neuron as a uniform sampler}

Lazar and Pnevmatikakis (2008a) have shown that, for an input $u \in P W_{\Omega}$, functions $\left\{\phi_{k}^{\mathcal{T}_{u}}\right\}_{k \in \mathbb{Z}}$, corresponding to the $t$-transform equation (2.4) of the ideal IF neuron, form a frame for $P W_{\Omega}$ provided that $\exists \Delta>0$ such that $\mathcal{T}_{u}$ is $\Delta$ - dense and $\Delta<\frac{\pi}{\Omega}$.

For a generic input $u \in P W_{\Omega}$, the set of frame functions $\left\{\phi_{k}^{\mathcal{T}_{u}}\right\}_{k \in \mathbb{Z}}$ is determined by the non-uniform sequence $\mathcal{T}_{u}=\left\{t_{k}\right\}_{k \in \mathbb{Z}}$, and thus depends on function $u$. In the following, it will be shown that the nonuniform sampling operation performed by an IF neuron with parameters $\bar{\delta}$ and $b$ can be reformulated as one of uniform sampling at points $\mathcal{D}=\{k \bar{\delta}\}_{k \in \mathbb{Z}}$ for an auxiliary function $\bar{\psi}^{\prime}: \mathbb{R} \rightarrow \mathbb{R}$, uniquely determined from input $u$. The following theorem defines function $\bar{\psi}^{\prime}$ and establishes some of its important properties. 
Theorem 3.2. Let $\mathcal{T}_{u}=\left\{t_{k}\right\}_{k \in \mathbb{Z}}$ be the spike times sequence generated by an ideal IF neuron with parameters $\{b, \bar{\delta}\}$, for a given input function $u \in L^{2}(\mathbb{R}) \cap C(\mathbb{R}),|u(t)| \leq$ $c<b, \forall t \in \mathbb{R}$. Let $y(t) \triangleq \int_{0}^{t}(u(\tau)+b) d \tau$. It follows that $y$ admits an inverse $\psi$ that satisfies the following

A) $\mathcal{L}_{k}^{\mathcal{D}} \bar{\psi}^{\prime}=\bar{q}_{k}, \forall k \in \mathbb{Z}$,

where $\bar{\psi}(x) \triangleq \psi(x)-x / b, \mathcal{D}=\{k \bar{\delta}\}_{k \in \mathbb{Z}}$ and $\bar{q}_{k} \triangleq\left(t_{k+2}-t_{k+1}\right)-\bar{\delta} / b, \forall k \in \mathbb{Z}$,

B) $\left|\bar{\psi}^{\prime}(x)\right| \leq \frac{c}{b(b-c)}, \forall x \in \mathbb{R}$ and $\left\|\bar{\psi}^{\prime}\right\|_{L^{2}}^{2} \leq \frac{1}{b^{2}(b-c)}\|u\|_{L^{2}}^{2}$,

where $\|\cdot\|_{L^{2}}$ denotes the standard norm in $L^{2}(\mathbb{R})$.

Proof. A) From its definition, $y$ is strictly increasing and continuous. Furthermore

$$
y(t) \geq(b-c) t, \forall t \geq 0, y(t) \leq(b-c) t, \forall t<0 \Rightarrow \lim _{t \rightarrow \pm \infty} y(t)= \pm \infty .
$$

It follows that $y$ is one-to-one and onto function. Thus it has an inverse $\psi: \mathbb{R} \rightarrow \mathbb{R}$. From (2.3) and the definition of $y$, it follows that $y\left(t_{k+1}\right)=k \bar{\delta}, \forall k \in \mathbb{Z}$, and $\psi(k \bar{\delta})=t_{k+1}, \forall k \in \mathbb{Z}$. Consequently,

$$
\bar{\psi}(k \bar{\delta})=\psi(k \bar{\delta})-k \bar{\delta} / b=t_{k+1}-k \bar{\delta} / b, \forall k \in \mathbb{Z}
$$

The proof follows from the definition of operator $\mathcal{L}_{k}^{\mathcal{D}}$ and equations (3.9).

B) By applying the Inverse Function Theorem, it follows that $\psi^{\prime}(y(t))=1 / y^{\prime}(t)$.

Furthermore,

$$
\bar{\psi}^{\prime}(y(t))=-\frac{u(t)}{b(u(t)+b)}
$$

It follows that

$$
\max _{x \in \mathbb{R}}\left|\bar{\psi}^{\prime}(x)\right|=\max _{u \in[-c, c]} \frac{1}{b}\left|\frac{u}{u+b}\right|=\frac{c}{b(b-c)}
$$

and

$$
\int_{-\infty}^{\infty} \bar{\psi}^{\prime 2}(x) d x=\int_{-\infty}^{\infty} \bar{\psi}^{\prime 2}(y(t)) y^{\prime}(t) d t=\int_{-\infty}^{\infty} \frac{u^{2}(t)}{b^{2}(u(t)+b)} d t \leq \frac{1}{b^{2}(b-c)}\|u\|_{L^{2}}^{2} .
$$

Corollary 3.2. Let $u \in L^{2}(\mathbb{R}) \cap C(\mathbb{R}),|u(t)| \leq c<b, b>0, \forall t \in \mathbb{R}$ and let $\psi(x)$ be the inverse of $y(t)=\int_{0}^{t}(u(\tau)+b) d \tau$. Then the following holds

$$
u(\psi(x))=-\frac{b^{2} \bar{\psi}^{\prime}(x)}{b \bar{\psi}^{\prime}(x)+1}
$$


where $\bar{\psi}(x)=\psi(x)-x / b$.

Proof. Follows from equation (3.10) by applying the change of variable $t=\psi(x)$.

Let $U: \mathbb{R} \rightarrow \mathbb{R}, U(t) \triangleq y(t)-b t=\int_{0}^{t} u(\tau) d \tau$. According to Theorem 3.2, it follows that the nonuniform samples $\left\{U\left(t_{k}\right)\right\}_{k \in \mathbb{Z}}$ and uniform samples $\{\bar{\psi}(k \bar{\delta})\}_{k \in \mathbb{Z}}$ can be calculated from the output sequence $\mathcal{T}_{u}$ of an IF neuron as

$$
U\left(t_{k}\right)=k \bar{\delta}-b t_{k}, \bar{\psi}(k \bar{\delta})=t_{k}-k \bar{\delta} / b, \forall k \in \mathbb{Z}
$$

The relationship between functions $U$ and $\bar{\psi}$ is depicted in Figure 3.1, for an input function $u(t)=\operatorname{sinc}(2 \pi \cdot 80 t)$ and an IF neuron with parameters $b=2$ and $\bar{\delta}=8 \cdot 10^{-3}$.
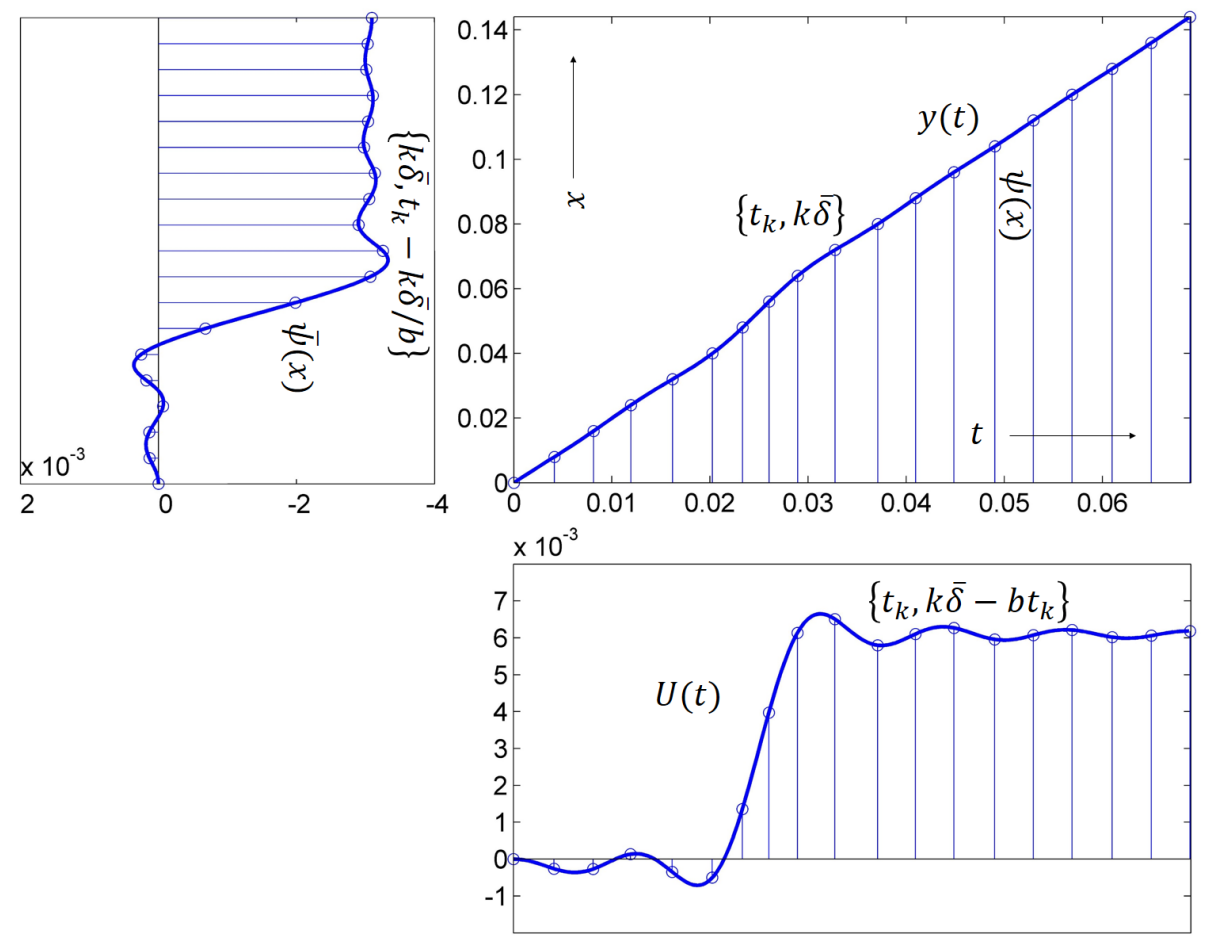

Figure 3.1: The relationship between functions $U$ and $\psi$ for a bandlimited input function.

From (3.10), the function $\bar{\psi}^{\prime}$ can be written as

$$
\bar{\psi}^{\prime}=p \circ u \circ \psi,
$$

where $p:[-c, c] \rightarrow \mathbb{R}, p(x)=-\frac{x}{b(x+b)}$.

In general, function $\bar{\psi}^{\prime}$ is not bandlimited, i.e., $\bar{\psi}^{\prime} \notin P W_{\Omega^{*}}, \forall \Omega^{*}>0$. For a function $u \in P W_{\Omega}$, the composition $u \circ \psi$ is a time-warped function (Cochran and Clark, 1990), 
whose exact bandwidth formula is yet to be derived for the general case. A new bandwidth can be calculated for this type of function, called essential maximum frequency or the essential bandwidth, which is defined as the bandwidth where most of the signal energy resides (Bergner et al., 2006, Do et al., 2012)

$$
\operatorname{ess} B W_{u \circ \psi}=\Omega \cdot \max _{x \in \mathbb{R}}\left|\psi^{\prime}(x)\right|,
$$

where $\operatorname{ess} B W_{u \circ \psi}$ denotes the essential bandwidth of function $u \circ \psi$.

The following auxiliary lemma is required for calculating the essential bandwidth of $\psi^{\prime}$.

Lemma 3.2. Let $u_{p} \triangleq p \circ u$, where $u \in P W_{\Omega},|u| \leq c<b$, and $p:[-c, c] \rightarrow \mathbb{R}, p(x)=$ $-\frac{x}{b(x+b)}$. Then, $\forall M \in \mathbb{N}, \exists u_{p, M}, v_{p, M}$ such that

$$
\begin{aligned}
u_{p} & =u_{p, M}+v_{p, M}, \\
u_{p, M} & \in P W_{M \Omega}, \\
\left\|v_{p, M}\right\|_{L^{2}}^{2} & \leq\left(\frac{c}{b}\right)^{2 M}\left\|u_{p}\right\|_{L^{2}}^{2} .
\end{aligned}
$$

Proof. By using the expansion of $p$ in Taylor series around 0 , it follows that

$$
u_{p}(t)=-\frac{u(t)}{b^{2}}+\frac{u^{2}(t)}{b^{3}}-\cdots+(-1)^{M} \frac{u^{M}(t)}{b^{M+1}}+R_{M}(u(t)),
$$

where

$$
R_{M}(x)=p(x)\left(-\frac{x}{b}\right)^{M}
$$

Let $v_{p, M} \triangleq R_{M} \circ u$ and $u_{p, M} \triangleq u_{p}-v_{p, M}$. Using $|u(t)|<c, \forall t \in \mathbb{R}$, it follows that

$$
\left\|v_{p, M}\right\|_{L^{2}}^{2}=\int_{-\infty}^{\infty} u_{p}^{2}(t)\left(-\frac{u(t)}{b}\right)^{2 M} d t \leq\left(\frac{c}{b}\right)^{2 M}\left\|u_{p}\right\|_{L^{2}}^{2}
$$

From the Convolution Theorem for Fourier transform, by induction, it follows that

$$
\widehat{u^{M}}=\widehat{u} * \widehat{u} * \underset{(M \text { times })}{\ldots} * \widehat{u}
$$

where $\widehat{u}$ stands for the Fourier transform of function $u$ and $*$ denotes the convolution operator. Using Titchmarsh's convolution theorem in (3.17), it follows that $u^{M} \in P W_{M \Omega}$ (Titchmarsh, 1926) and therefore $u_{p, M} \in P W_{M \Omega}$.

The following lemma shows that $\bar{\psi}^{\prime}$ can be approximated with arbitrary precision by a function with finite essential bandwidth and defines a bound for the approximation error. 
Lemma 3.3. For any $M \in \mathbb{N}$, function $\bar{\psi}^{\prime}$ can be decomposed as

$$
\bar{\psi}^{\prime}=\bar{\psi}_{M}^{\prime}+e_{M}
$$

where

$$
\begin{aligned}
\operatorname{ess} B W_{\bar{\psi}_{M}^{\prime}} & \leq \frac{M \Omega}{b-c} \\
\left\|e_{M}\right\|_{L^{2}}^{2} & \leq\left(\frac{c}{b}\right)^{2 M} \frac{b+c}{b-c}\left\|\bar{\psi}^{\prime}\right\|_{L^{2}}^{2} .
\end{aligned}
$$

Proof. Using Lemma 3.2 and equation (3.13), it follows that, for any $M \in \mathbb{N}, \exists u_{p, M}$ and $v_{p, M}$ such that

$$
\bar{\psi}^{\prime}=u_{p, M} \circ \psi+v_{p, M} \circ \psi,
$$

where $u_{p, M} \in P W_{M \Omega}$ and $\left\|v_{p, M}\right\|_{L^{2}}^{2} \leq\left(\frac{c}{b}\right)^{2 M}\left\|u_{p}\right\|_{L^{2}}^{2}$. Let $\bar{\psi}_{M}{ }^{\prime} \triangleq u_{p, M} \circ \psi$ and $e \triangleq$ $v_{p, M} \circ \psi$.

From equation (3.10), it follows that $\psi^{\prime}(x)>1 /(b+c), \forall x \in \mathbb{R}$, since $|u(t)|<$ $c, \forall t \in \mathbb{R}$. Then the following hold

$$
\begin{aligned}
\operatorname{ess} B W_{\bar{\psi}_{M}^{\prime}} & =\max _{x \in \mathbb{R}}\left|\psi^{\prime}\right| \cdot M \Omega \leq \frac{M \Omega}{b-c} \\
\|e\|_{L^{2}}^{2} & =(b+c) \int_{\mathbb{R}}\left(v_{p, M}(\psi(x))\right)^{2} \frac{1}{b+c} d x \\
& \leq(b+c) \int_{\mathbb{R}}\left(v_{p, M}(\psi(x))\right)^{2} \psi^{\prime}(x) d x \\
& =(b+c)\left\|v_{p, M}\right\|_{L^{2}}^{2} \\
& \leq(b+c)\left(\frac{c}{b}\right)^{2 M}\left\|u_{p}\right\|_{L^{2}}^{2} .
\end{aligned}
$$

Using the boundedness of $\psi^{\prime}$, it follows that

$$
\left\|u_{p}\right\|_{L^{2}}^{2}=\int_{\mathbb{R}}\left(u_{p}(\psi(x))\right)^{2} \psi^{\prime}(x) d x \leq \int_{\mathbb{R}}\left(u_{p}(\psi(x))\right)^{2} \frac{1}{b-c} d x=\frac{1}{b-c}\left\|\bar{\psi}^{\prime}\right\|_{L^{2}}^{2}
$$

Inequality (3.23) is then used in (3.22) to complete the proof. 


\subsection{Fast indirect reconstruction algorithms for inputs encoded with ideal IF neurons}

According to Theorem 3.2, for any function $u \in P W_{\Omega} \subset L^{2}(\mathbb{R})$ and for any IF neuron with parameters $\bar{\delta}$ and $b$, the function $\bar{\psi}^{\prime}$ satisfies

$$
\mathcal{L}_{k}^{\mathcal{D}} \bar{\psi}^{\prime}=\bar{q}_{k}, \forall k \in \mathbb{Z},
$$

where $\bar{\psi}(x)=\psi(x)-x / b, \psi$ is the inverse of $y(t)=\int_{0}^{t}(u(\tau)+b) d \tau, \mathcal{D}=\{k \bar{\delta}\}_{k \in \mathbb{Z}}$, and $\bar{q}_{k}=\left(t_{k+2}-t_{k+1}\right)-\bar{\delta} / b$. Given that the function $\bar{\psi}^{\prime}$ can be approximated with arbitrary precision by a $\bar{\psi}_{M}^{\prime}$, whose essential bandwidth is bounded by $\bar{\Omega}_{M}=\frac{M \Omega}{b-c}$, the function $\bar{\psi}^{\prime}$ is reconstructed in the space $P W_{\bar{\Omega}_{M}}$.

Sequence $\mathcal{D}=\{k \bar{\delta}\}_{k \in \mathbb{Z}}$ is $\bar{\delta}$ - dense and relatively separated. Therefore the function $\bar{\psi}^{\prime}$ can be reconstructed using Theorem 3.1 from sequence $\left\{\mathcal{L}_{k}^{\mathcal{D}} \bar{\psi}^{\prime}\right\}_{k \in \mathbb{Z}}$ provided that $\bar{\delta}<\frac{\pi}{\bar{\Omega}_{M}}$. The reconstruction formulas for functions $\bar{\psi}^{\prime}$ and $\bar{\psi}$ are presented in the following theorem.

Theorem 3.3. Let $\bar{\psi}^{\prime} \in P W_{\bar{\Omega}_{M^{\prime}}}$ where $\bar{\Omega}_{M}=\frac{M \Omega}{b-c}$, and let $\mathcal{D}=\{k \bar{\delta}\}_{k \in \mathbb{Z}}$, such that

$$
\bar{\delta}<\frac{\pi}{\bar{\Omega}_{M}} .
$$

Then $\bar{\psi}^{\prime}$ and $\bar{\psi}$ can be reconstructed iteratively from $\left\{\mathcal{L}_{k}^{\mathcal{D}} \bar{\psi}^{\prime}\right\}_{k \in \mathbb{Z}}$ as

$$
\begin{aligned}
& \bar{\psi}^{\prime}=\lim _{n \rightarrow \infty} \bar{\psi}_{n}^{\prime}=\lim _{n \rightarrow \infty} \overline{\boldsymbol{g}}_{1}^{T} \overline{\boldsymbol{P}}_{n} \overline{\boldsymbol{q}} \\
& \bar{\psi}=\lim _{n \rightarrow \infty} \bar{\psi}_{n}=\lim _{n \rightarrow \infty} \overline{\boldsymbol{g}}_{2}^{T} \overline{\boldsymbol{P}}_{n} \overline{\boldsymbol{q}}
\end{aligned}
$$

where $\left[\overline{\boldsymbol{g}}_{1}\right]_{m}=g_{\bar{\Omega}_{M}}\left(\cdot-\bar{s}_{m+1}\right), \overline{\boldsymbol{s}}_{m} \triangleq \frac{2 m-1}{2} \bar{\delta},\left[\overline{\boldsymbol{g}}_{2}\right]_{m}=R_{\bar{s}_{m+1}}, R_{\bar{s}_{m+1}}(x)=\int_{0}^{x} g_{\bar{\Omega}_{M}}(\tau-$ $\left.\bar{s}_{m+1}\right) d \tau, \forall x \in \mathbb{R},[\overline{\boldsymbol{G}}]_{m, k}=\mathcal{L}_{m}^{\mathcal{D}}\left(g_{\bar{\Omega}_{M}}\left(\cdot-\bar{s}_{k+1}\right)\right), \overline{\boldsymbol{P}}_{n}=\sum_{p=0}^{n}(\boldsymbol{I}-\overline{\boldsymbol{G}})^{p}, \forall n \in \mathbb{N}$, and $[\overline{\boldsymbol{q}}]_{k}=\mathcal{L}_{k}^{\mathcal{D}} \bar{\psi}^{\prime}, \forall k, m \in \mathbb{Z}$. Moreover,

$$
\left\|\bar{\psi}^{\prime}-\bar{\psi}_{n}^{\prime}\right\|_{L^{2}} \leq\left(\frac{\bar{\delta} \bar{\Omega}_{M}}{\pi}\right)^{n+1}\left\|\bar{\psi}^{\prime}\right\|_{L^{2}}, \forall n \in \mathbb{N}
$$

Proof. The proof follows from Theorem 3.1 and Corollary 3.1.

Samples $\left\{\mathcal{L}_{k}^{\mathcal{D}} \bar{\psi}^{\prime}\right\}_{k \in \mathbb{Z}}$ depend on uniform sequence $\mathcal{D}$, and thus the problem of recovering $u$ has been redefined as one of reconstruction from uniform samples (Shannon, 1949).

Corollary 3.3. Under the assumptions of Theorem 3.3, functions $\bar{\psi}^{\prime}$ and $\bar{\psi}$ can be recon- 
structed as

$$
\begin{aligned}
& \bar{\psi}^{\prime}=\overline{\boldsymbol{g}}_{1}^{T} \overline{\boldsymbol{G}}^{+} \overline{\boldsymbol{q}}, \\
& \bar{\psi}=\overline{\boldsymbol{g}}_{2}^{T} \overline{\boldsymbol{G}}^{+} \overline{\boldsymbol{q}} .
\end{aligned}
$$

Proof. The proof follows from Corollary 3.1.

Remark 1. According to Lemma 3.3, increasing $M$ reduces the approximation error (3.25), increases the bandwitdh $\bar{\Omega}_{M}$, and, as a consequence, requires a smaller $\bar{\delta}$ for condition (3.24) to be satisfied. Through numerical simulations it was found that for a value of $M=$ 2 the error introduced by approximation (3.18) is comparable to the common numerical errors.

Functions $\bar{\psi}$ and $\bar{\psi}^{\prime}$, given by (3.26) and (3.27), are computed on the uniform grid $\{l \varepsilon\}_{l=0, \ldots, \bar{L}}$, where $\varepsilon$ is the sampling period used in reconstruction. The reconstructed signal $\widetilde{u}$ is the interpolation of $u$ at points $\{\psi(l \varepsilon)\}_{l=0, \ldots, \bar{L}}$, where values $\{u(\psi(l \varepsilon))\}_{l=0, \ldots, \bar{L}}$ are given by (3.12). Using piecewise linear interpolation gives

$$
\widetilde{u}(t)=u(\psi(l \varepsilon))+\frac{t-\psi(l \varepsilon)}{\psi((l+1) \varepsilon)-\psi(l \varepsilon)} \cdot[u(\psi((l+1) \varepsilon))-u(\psi(l \varepsilon))],
$$

for $\forall l=0, \ldots, \bar{L}, \forall t \in[\psi(l \varepsilon), \psi((l+1) \varepsilon)[$.

As it will be shown in Section 3.5, increasing the interpolation degree does not increase significantly the accuracy of the proposed method.

In the following, two algorithms are given for reconstructing a function $u$ over the interval $\left[t_{1}, t_{N+1}\right]$ from a finite number of time samples $\mathcal{T}_{u}=\left\{t_{k}\right\}_{k=1, \ldots, N+1}$. The two algorithms are based on Theorem 3.3 and Corollary 3.3, respectively.

\section{Algorithm 3.1.}

Step 1. Calculate $\bar{G}, g_{\bar{\Omega}_{M}}\left(l \varepsilon-\bar{s}_{m+1}\right), R_{\bar{s}_{m+1}}(l \varepsilon), l=0, \ldots, \bar{L}$, where $\bar{\Omega}_{M}=\frac{M \Omega}{b-c}$ and

$$
\begin{gathered}
g_{\bar{\Omega}_{M}}\left(l \varepsilon-\bar{s}_{m+1}\right)=\frac{\sin \left(\bar{\Omega}_{M}\left(l \varepsilon-\bar{s}_{m+1}\right)\right)}{\pi\left(l \varepsilon-\bar{s}_{m+1}\right)}, \\
{[\overline{\boldsymbol{G}}]_{k m}=\int_{k \delta}^{(k+1) \delta} g_{\bar{\Omega}_{M}}\left(\tau-\bar{s}_{m+1}\right) d \tau,} \\
R_{\bar{s}_{m+1}}(l \varepsilon)=\int_{0}^{l \varepsilon} g_{\bar{\Omega}_{M}}\left(\tau-\bar{s}_{m+1}\right) d \tau, k, m=1, \ldots, N ;
\end{gathered}
$$

Step 2. Calculate $\overline{\boldsymbol{P}}_{n}$ iteratively as

$$
\begin{gathered}
\overline{\boldsymbol{P}}_{0}=\boldsymbol{I} ; \\
\overline{\boldsymbol{P}}_{j}=\boldsymbol{I}+\overline{\boldsymbol{P}}_{j-1}(\boldsymbol{I}-\overline{\boldsymbol{G}}), j=1, \ldots, n ;
\end{gathered}
$$


Step 3. Calculate $\bar{q}$

$$
[\overline{\boldsymbol{q}}]_{m}=\left(t_{m+2}-t_{m+1}\right)-\bar{\delta} / b, m=1, \ldots, N
$$

Step 4. Calculate $\bar{c}$

$$
\overline{\boldsymbol{c}}=\overline{\boldsymbol{P}}_{n} \overline{\boldsymbol{q}}
$$

Step 5. Calculate $\bar{\psi}_{r e c}^{\prime}(l \varepsilon), \bar{\psi}_{r e c}(l \varepsilon)$, and $\psi_{r e c}(l \varepsilon), l=0, \ldots, \bar{L}$, where

$$
\begin{gathered}
\bar{\psi}_{r e c}{ }^{\prime}(l \varepsilon)=\sum_{m=1}^{N} c_{m} g_{\bar{\Omega}_{M}}\left(l \varepsilon-\bar{s}_{m+1}\right), \\
\bar{\psi}_{r e c}(l \varepsilon)=\sum_{m=1}^{N} c_{m} R_{\bar{s}_{m+1}}(l \varepsilon), \\
\psi_{r e c}(l \varepsilon)=\bar{\psi}_{r e c}(l \varepsilon)+l \varepsilon / b ;
\end{gathered}
$$

Step 6. Calculate

$$
u_{r e c}\left(\psi_{r e c}(l \varepsilon)\right)=-\frac{b^{2} \bar{\psi}_{r e c}{ }^{\prime}(l \varepsilon)}{b \bar{\psi}_{r e c}{ }^{\prime}(l \varepsilon)+1}, l=0, \ldots, \bar{L}
$$

Step 7. Calculate $\widetilde{u}_{r e c}$, the interpolation of $u_{r e c}$ at points $\left\{\psi_{r e c}(l \varepsilon)\right\}_{l=0, \ldots, \bar{L}}$, and sample it at points $\{j \varepsilon\}_{j=0, \ldots, L}$. For the particular case of piecewise linear interpolation, the expression of $\widetilde{u}$ is

$$
\begin{aligned}
& \widetilde{u}_{r e c}(j \varepsilon)= u_{r e c}\left(\psi_{r e c}\left(l_{j} \varepsilon\right)\right)+\frac{j \varepsilon-\psi_{r e c}\left(l_{j} \varepsilon\right)}{\psi_{r e c}\left(\left(l_{j}+1\right) \varepsilon\right)-\psi_{r e c}\left(l_{j} \varepsilon\right)} \\
& \cdot\left[u\left(\psi_{r e c}\left(\left(l_{j}+1\right) \varepsilon\right)\right)-u_{r e c}\left(\psi_{r e c}\left(l_{j} \varepsilon\right)\right)\right]
\end{aligned}
$$

for $j=0, \ldots, L$, where $l_{j} \in \mathbb{Z}$ satisfies $\psi_{r e c}\left(l_{j} \varepsilon\right) \leq j \varepsilon<\psi_{r e c}\left(\left(l_{j}+1\right) \varepsilon\right)$.

The proposed non-iterative algorithm based on Corollary 3.3 is presented as follows. The algorithm is an alternative to the state-of-the-art method in (Lazar and Pnevmatikakis, 2008a).

\section{Algorithm 3.2.}

Step 1. Calculate $\bar{G}, g_{\bar{\Omega}_{M}}\left(l \varepsilon-\bar{s}_{m+1}\right), R_{\bar{s}_{m+1}}(l \varepsilon), l=0, \ldots, \bar{L}$, where $\bar{\Omega}_{M}=\frac{M \Omega}{b-c}$ and

$$
\begin{gathered}
g_{\bar{\Omega}_{M}}\left(l \varepsilon-\bar{s}_{m+1}\right)=\frac{\sin \left(\bar{\Omega}_{M}\left(l \varepsilon-\bar{s}_{m+1}\right)\right)}{\pi\left(l \varepsilon-\bar{s}_{m+1}\right)}, \\
{[\overline{\boldsymbol{G}}]_{k m}=\int_{k \delta}^{(k+1) \delta} g_{\bar{\Omega}_{M}}\left(\tau-\bar{s}_{m+1}\right) d \tau,} \\
R_{\bar{s}_{m+1}}(l \varepsilon)=\int_{0}^{l \varepsilon} g_{\bar{\Omega}_{M}}\left(\tau-\bar{s}_{m+1}\right) d \tau, k, m=1, \ldots, N ;
\end{gathered}
$$

Step 2. Calculate $\bar{G}^{+}$, the Moore-Penrose pseudoinverse of $\bar{G}$; 
Step 3. Calculate $\bar{q}$

$$
[\overline{\boldsymbol{q}}]_{m}=\left(t_{m+2}-t_{m+1}\right)-\bar{\delta} / b, m=1, \ldots, N
$$

Step 4. Calculate $\bar{c}$

$$
\bar{c}=\bar{G}^{+} \bar{q}
$$

Step 5. Calculate $\bar{\psi}_{\text {rec }}^{\prime}(l \varepsilon), \bar{\psi}_{\text {rec }}(l \varepsilon)$, and $\psi_{\text {rec }}(l \varepsilon), l=0, \ldots, \bar{L}$, where

$$
\begin{gathered}
\bar{\psi}_{r e c}{ }^{\prime}(l \varepsilon)=\sum_{m=1}^{N} c_{m} g_{\bar{\Omega}_{M}}\left(l \varepsilon-\bar{s}_{m+1}\right), \\
\bar{\psi}_{r e c}(l \varepsilon)=\sum_{m=1}^{N} c_{m} R_{\bar{s}_{m+1}}(l \varepsilon) \\
\psi_{r e c}(l \varepsilon)=\bar{\psi}_{r e c}(l \varepsilon)+l \varepsilon / b
\end{gathered}
$$

Step 6. Calculate

$$
u_{r e c}(\psi(l \varepsilon))=-\frac{b^{2} \bar{\psi}_{r e c}{ }^{\prime}(l \varepsilon)}{b \bar{\psi}_{r e c}{ }^{\prime}(l \varepsilon)+1}, l=0, \ldots, \bar{L}
$$

Step 7. Calculate $\widetilde{u}_{r e c}$, the interpolation of $u_{\text {rec }}$ at points $\left\{\psi_{r e c}(l \varepsilon)\right\}_{l=0, \ldots, \bar{L}}$, and sample it at points $\{j \varepsilon\}_{j=0, \ldots, L}$. For the particular case of piecewise linear interpolation, the expression of $\widetilde{u}$ is

$$
\begin{gathered}
\widetilde{u}_{r e c}(j \varepsilon)=u_{r e c}\left(\psi_{r e c}\left(l_{j} \varepsilon\right)\right)+\frac{j \varepsilon-\psi_{r e c}\left(l_{j} \varepsilon\right)}{\psi_{r e c}\left(\left(l_{j}+1\right) \varepsilon\right)-\psi_{r e c}\left(l_{j} \varepsilon\right)} \\
\cdot\left[u\left(\psi_{r e c}\left(\left(l_{j}+1\right) \varepsilon\right)\right)-u_{r e c}\left(\psi_{r e c}\left(l_{j} \varepsilon\right)\right)\right], \\
\text { for } j=0, \ldots, L, \text { where } l_{j} \in \mathbb{Z} \text { satisfies } \psi_{r e c}\left(l_{j} \varepsilon\right) \leq j \varepsilon<\psi_{r e c}\left(\left(l_{j}+1\right) \varepsilon\right) .
\end{gathered}
$$

The algorithms above calculate matrices $\overline{\boldsymbol{P}}_{n}$ and $\overline{\boldsymbol{G}}^{+}$, respectively, and the values of functions $g_{\bar{\Omega}_{M}}\left(\cdot-\bar{s}_{m+1}\right)$ and $R_{\bar{s}_{m+1}}, m=1, \ldots, N$, off-line, in steps 1 and 2 . Processing subsequent sets of spike times for every new set $h$ of spikes $\left\{t_{1}^{h}, t_{2}^{h}, \ldots, t_{N+1}^{h}\right\}$ involves only a few additions and multiplications to recover input $u$ on time interval $\left[t_{1}^{h}, t_{N+1}^{h}\right]$ (steps 3-7). Moreover, unlike the iterative algorithm proposed in (Lazar, 2004), the computation time of Algorithm 3.1 is not dependent on the number of computed iterations $n$.

\subsection{Numerical study}

In this section, the standard algorithms in (Lazar, 2004) and (Lazar and Pnevmatikakis, 2008a) are compared through numerical simulations with the two proposed algorithms.

Specifically, the performance of the algorithms is characterised in terms of reconstruction accuracy and computation time. The accuracy is measured by computing the signal-to- 
error ratio (SER)

$$
S E R=10 \log _{10}\left(\frac{\|u\|_{L^{2}}^{2}}{\left\|u-u_{\text {rec }}\right\|_{L^{2}}^{2}}\right),
$$

where $u, u_{r e c}$ denote the original and reconstructed signals, respectively. Furthermore, the distribution of the error along the time axis is evaluated using the following function

$$
E(t) \triangleq \frac{\left|u(t)-u_{r e c}(t)\right|}{\|u\|_{L^{2}}} \cdot 100
$$

The computation time was evaluated only for the routine performing the reconstruction rather than for the whole software implementation. The dependence of the approximation error (3.19) on the choice of parameter $M$ is investigated for the two proposed algorithms.

The accuracy, as a function of number of iterations, is illustrated for Algorithm 3.1 and the method in (Lazar, 2004). The relationship between computation time and the length of the sampling time sequence $\mathcal{T}_{u}=\left\{t_{k}\right\}_{k=1, \ldots, N+1}$ is also characterised for the proposed, as well as the standard algorithms (Lazar, 2004, Lazar and Pnevmatikakis, 2008a). Simulations were carried out in Matlab ${ }^{\circledR}$ Version 7.5.0.342 on a 3.1 GHz Intel Single Core PC workstation.

A set of 100 bandlimited signals $\left\{u^{j}\right\}_{j=1, \ldots, 100}$ were generated, where

$$
u^{j}(t)=\sum_{k=1}^{10} a_{j}^{k} \frac{\sin (\Omega(t-k T))}{\pi(t-k T)}, j=1, \ldots, 100, t \in[0,0.1],
$$

where $\Omega=2 \pi \cdot 80 \mathrm{~Hz}, T=\pi / \Omega$, and $a_{j}^{k}, k=1, \ldots 10$, are random coefficients drawn from the uniform distribution on $]-1,1\left[\right.$. The signals $u^{j}$ were uniformly sampled with sampling period $\varepsilon=4 \cdot 10^{-4}$. Every input was normalized such that $\max _{t \in \mathbb{R}}|u(t)|=c=$ 1. Inputs $\left\{u^{j}\right\}_{i=1,2,3}$ are depicted in Figure 3.2.

The signals were encoded by an ideal IF neuron with parameters $\delta=8 \cdot 10^{-3}, \mathrm{C}=$ $1, b=15$. Let $y^{j}(l \varepsilon) \triangleq \int_{0}^{l \varepsilon}\left(u^{j}(\tau)+b\right) d \tau, l=0, \ldots, \frac{0.1}{\varepsilon}$, where the integrals are computed using the trapezoid rule, and let $l_{k}$ be the unique solution of

$$
y^{j}\left(l_{k} \varepsilon\right) \leq k \bar{\delta}<y^{j}\left(\left(l_{k}+1\right) \varepsilon\right), \forall k=0, \ldots, 99
$$

Then $l_{k} \varepsilon$ is an approximation of the spike time number $k+1$ generated by the neuron. Here the spike times were approximated more accurately by generating sequences $\mathcal{T}_{u^{j}}=$ $\left\{t_{k}^{j}\right\}_{k=1, \ldots, 100}$ satisfying

$$
\begin{aligned}
k \bar{\delta} & =\alpha y^{i}\left(l_{k} \varepsilon\right)+(1-\alpha) y^{i}\left(\left(l_{k}+1\right) \varepsilon\right), \\
t_{k}^{j} & =\alpha l_{k} \varepsilon+(1-\alpha) \cdot\left(l_{k}+1\right) \varepsilon,
\end{aligned}
$$




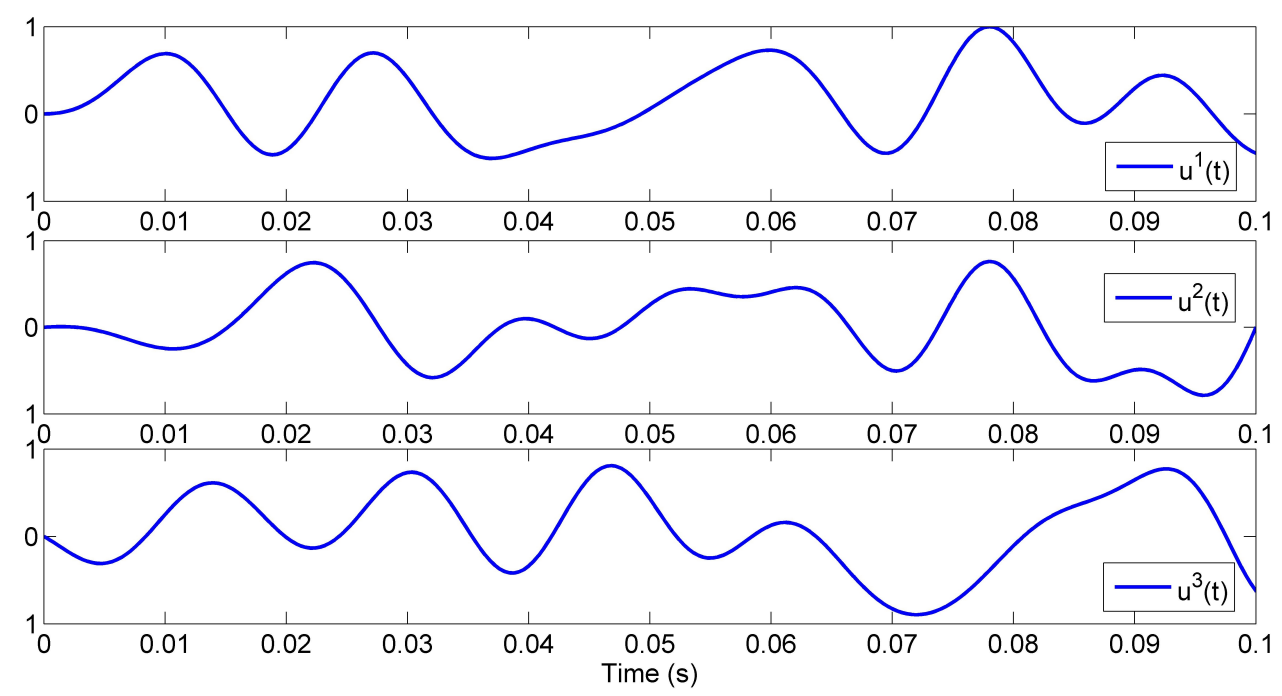

Figure 3.2: Input functions $\left\{u^{j}(t)\right\}_{i=1,2,3}$.

where $\alpha$ is an unknown variable.

The expression of $t_{k}^{j}$ is derived using $\alpha$ computed from (3.30)

$$
t_{k}^{j}=l_{k} \varepsilon+\varepsilon \cdot \frac{k \bar{\delta}-y^{j}\left(l_{k} \varepsilon\right)}{y^{j}\left(\left(l_{k}+1\right) \varepsilon\right)-y^{j}\left(l_{k} \varepsilon\right)}, j, k=1, \ldots, 100 .
$$

The parameters chosen satisfy the reconstruction conditions $\frac{\bar{\delta}}{b-c}<\frac{\pi}{\Omega}$ and $\bar{\delta}<\frac{(b-c) \pi}{M \Omega}$ for $M \leq 10$, which are required by the standard methods (Lazar, 2004, Lazar and Pnevmatikakis, 2008a) and Algorithm 3.1 and 3.2, respectively.

\subsubsection{Numerical study for Algorithm 3.1}

The first of the two proposed algorithms (Algorithm 3.1) is compared through numerical simulations with the standard iterative reconstruction algorithm presented in (Lazar, 2004).

The parameter $M \in \mathbb{N}^{*}$ determines the error of approximating function $\bar{\psi}^{\prime}$ by $\bar{\psi}_{M}^{\prime}$ (3.19), which is later on used in reconstruction. In order to investigate numerically the relationship between $M$ and the $S E R$, sequences $\left\{\mathcal{T}_{u^{j}}\right\}_{j=1, \ldots, 100}$ were used to reconstruct inputs $u^{j}, j=1, \ldots, 100$, for $M$ varying from 1 to 5 . Numerical simulations showed a decrease in accuracy for larger values.

For each value of $M$, the inputs $\left\{u^{j}\right\}_{j=1,100}$ were reconstructed using Algorithm 3.1 with $n=1$ and $n=1000$ iterations, respectively. Figure 3.3 displays the means and standard deviations of the SER values for each bandwidth $\bar{\Omega}_{M}=\frac{M \Omega}{b-c}$ of $\bar{\psi}_{M}{ }^{\prime}$. Performing a larger number of iterations improves the accuracy as shown in Figure 3.4. 


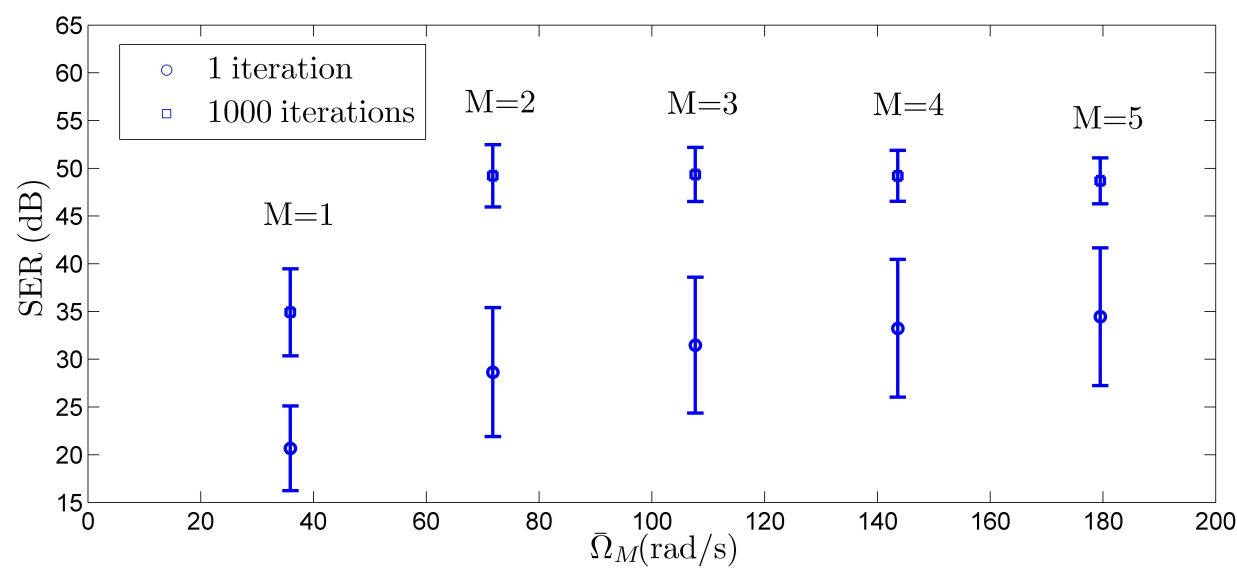

Figure 3.3: Reconstruction SER of Algorithm 3.1 for different values of $\bar{\Omega}_{M}$.

For $M \geq 2$ the $S E R$ saturates. Based on a few dozen numerical simulations carried out it can be concluded that $M=2$ can be used to achieve good results. According to (3.19), this corresponds to the following error bound for approximating function $\bar{\psi}^{\prime}$

$$
\left\|e_{M}\right\|_{L^{2}} \leq 2.25 \cdot 10^{-5}\left\|\bar{\psi}^{\prime}\right\|_{L^{2}}
$$

In order to determine how the $S E R$ is influenced by the number of iterations used in reconstruction, inputs $\left\{u^{j}\right\}_{j=1, \ldots, 100}$ were reconstructed with Algorithm 3.1 and the standard iterative algorithm (Lazar, 2004) for an increasing number of up to $10^{4}$ iterations. The average SER, calculated for each number of iterations for both methods, is depicted in Figure 3.4.

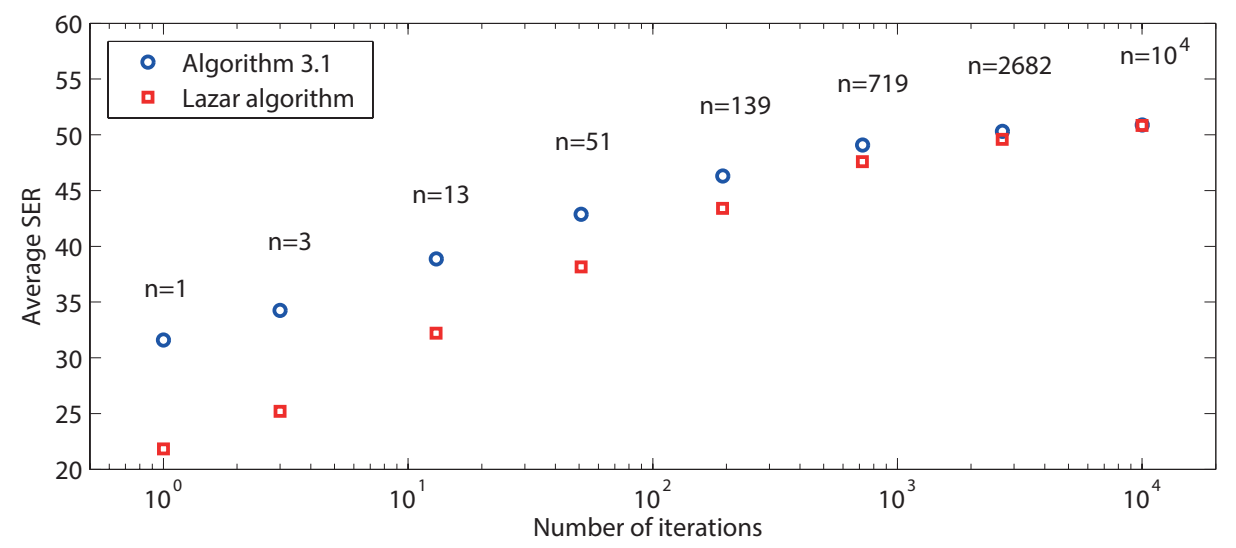

Figure 3.4: Average SER of 100 inputs reconstructed with Algorithm 3.1 and the standard algorithm for an increasing number of iterations. 

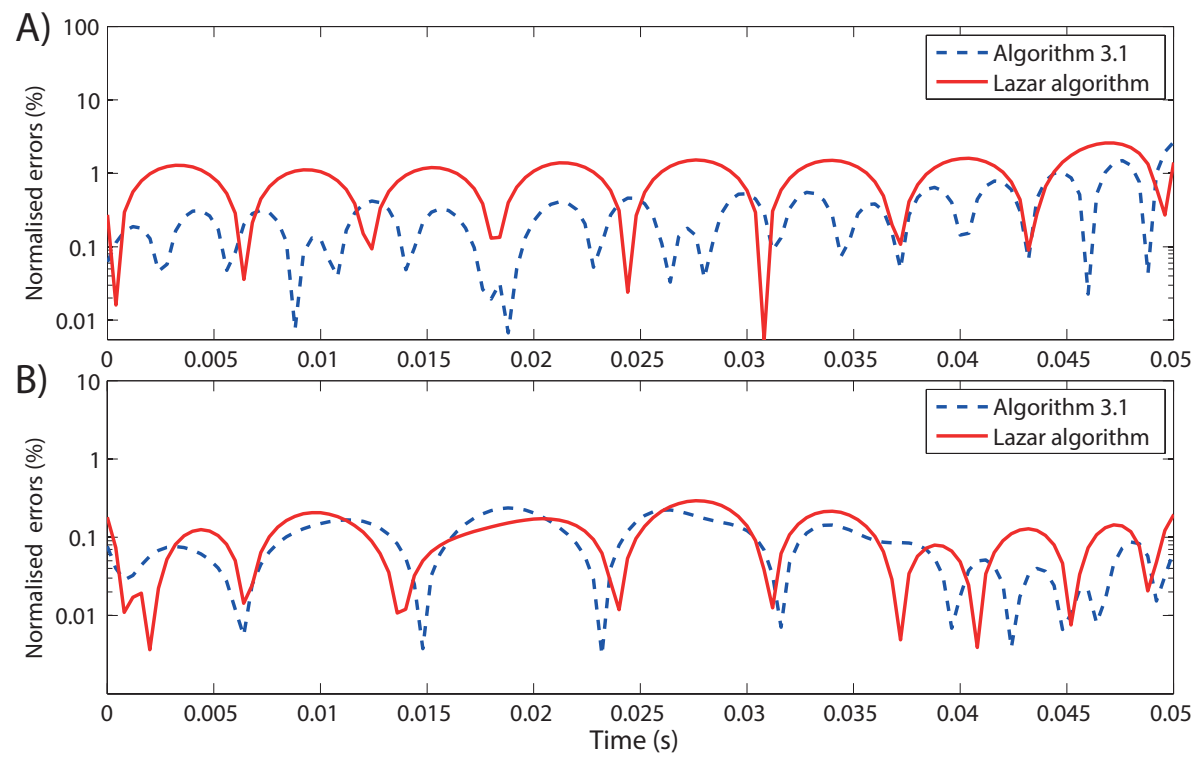

Figure 3.5: Normalised reconstruction errors $E^{1}(t)$ of Algorithm 3.1 and the standard algorithm corresponding to A) $n=1$ and B) $n=10^{4}$ iterations.

Figure 3.5 depicts the error function $E(t)$ (3.28) calculated for input $u^{1}(t)$, for both algorithms, for $n=1$ and $n=10^{4}$ iterations, respectively.

The results show that Algorithm 3.1 performs, on average, with higher accuracy for $n<10^{4}$. In the following, it will be shown that this is mainly caused by the difference in convergence speed to the solution of the linear system corresponding to each reconstruction algorithm.

Matrices $\overline{\boldsymbol{P}}_{n}$ and $\boldsymbol{P}_{n}$ from Algorithm 3.1 and Corollary 3.1, respectively, satisfy the following

$$
\lim _{n \rightarrow \infty} \overline{\boldsymbol{P}}_{n}=\overline{\boldsymbol{G}}^{+}, \lim _{n \rightarrow \infty} \boldsymbol{P}_{n}=\mathbf{G}^{+},
$$

where $\bar{G}$ and $G$ are the matrices of the following systems

$$
\bar{G} \bar{c}=\bar{q}, G c=q .
$$

To quantify the convergence of $\overline{\boldsymbol{P}}_{n}$ and $\boldsymbol{P}_{n}$, the normalised residual errors have been computed for the 100 spike times as

$$
r_{n} \triangleq \frac{\left\|\boldsymbol{G} \boldsymbol{P}_{n} \boldsymbol{q}-\boldsymbol{q}\right\|_{\mathbb{R}^{99}}}{\|\boldsymbol{q}\|_{\mathbb{R}^{99}}}, \bar{r}_{n} \triangleq \frac{\left\|\overline{\boldsymbol{G}} \overline{\boldsymbol{P}}_{n} \overline{\boldsymbol{q}}-\overline{\boldsymbol{q}}\right\|_{\mathbb{R}^{99}}}{\|\overline{\boldsymbol{q}}\|_{\mathbb{R}^{99}}}, \forall n \in \mathbb{N},
$$

where $\|\cdot\|_{\mathbb{R}^{99}}$ is the norm of vectors $\boldsymbol{v} \in \mathbb{R}^{99}$, defined as $\|\boldsymbol{v}\|_{\mathbb{R}^{99}} \triangleq \sqrt{\sum_{k=1}^{99}[\boldsymbol{v}]_{k}^{2}}$. The values of $r_{n}$ and $\bar{r}_{n}$ were displayed in Figure 3.6. 


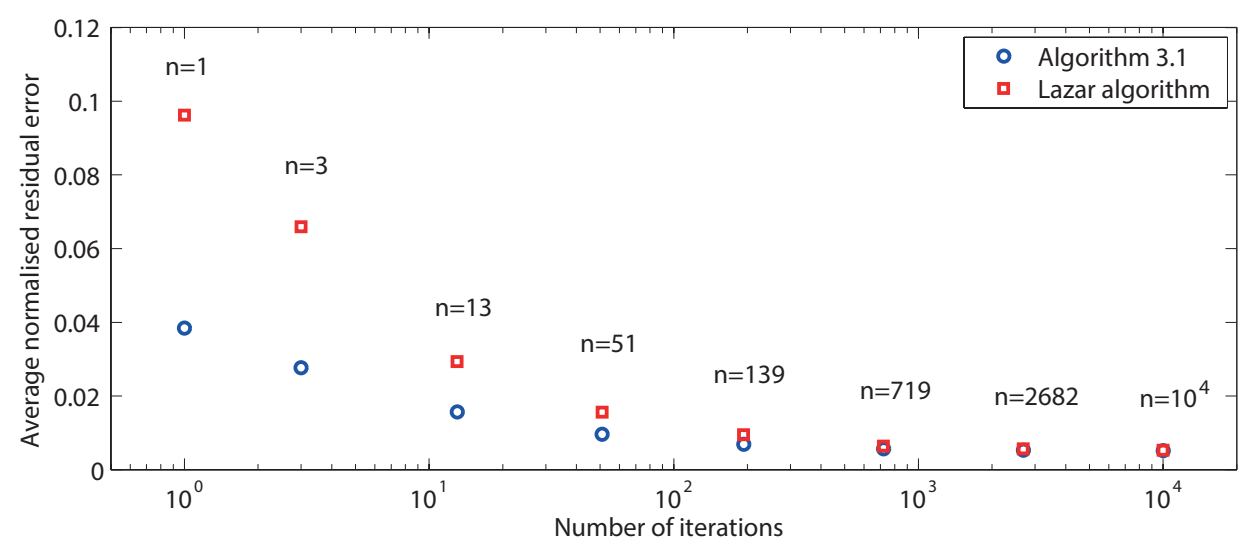

Figure 3.6: Normalised residual error of Algorithm 3.1 and the standard algorithm for an increasing number of iterations.

The inputs $\left\{u^{j}\right\}_{j=1, \ldots, 100}$ were reconstructed from the spike-time sequences $\left\{\mathcal{T}_{u^{j}}\right\}_{j=1, \ldots, 100}$ using Algorithm 3.1 and the iterative algorithm in (Lazar, 2004). The number of iterations used was $n=10^{3}$ and $n=10^{4}$. The performance of the algorithms was assessed by computing the $S E R$ and measuring the reconstruction time. According to the results depicted in Figure 3.7, the computing time for Algorithm 3.1 is more than 2 orders of magnitude shorter than the standard method and, unlike the latter, is not affected by the number of iterations used in reconstruction.

In order to investigate the effect of increasing the number of spikes on the reconstruction time, numerical simulations were carried out in which the input signal $u^{1}$ was reconstructed from spike time sequences $\left\{t_{k}^{1}\right\}_{k=1, \ldots, P}$, with lengths $P=25,50, \ldots, 400$, respectively, using both algorithms for different number of iterations. The reconstruction times for Algorithm 3.1 and the Lazar algorithm are shown in Figure 3.8.

The results show that Algorithm 3.1 is far less sensitive to the length of processed the spike time sequences, and insensitive to the number of iterations used in reconstruction. Specifically, for one iteration, the computation time corresponding to $N=400$ spikes increases 15 times for Algorithm 3.1 and 341 times for the proposed and the Lazar algorithm, respectively, compared to the time taken to process 25 spikes. For $10^{3}$ iterations, the computation time corresponding to the same number of spikes processed increases 27 and 929 times for the proposed algorithm and the standard method in (Lazar, 2004), respectively.

The step performing the iterations in Algorithm 3.1 is computed off-line, and thus the computation time is not dependent on the number of iterations. In contrast, the computing time of the standard method in (Lazar, 2004) is very sensitive to the number of iterations. Specifically, for $N=25$ and $N=400$ spike times, the computation time corresponding to $10^{3}$ iterations increases 3 and 9 times, respectively, compared to the time take to process 
A1)

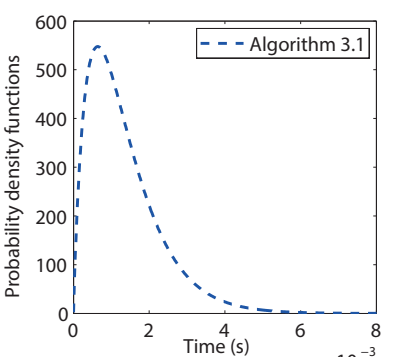

B1)

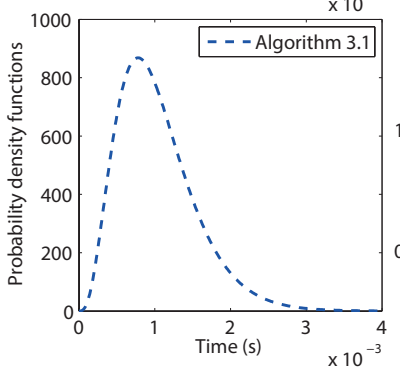

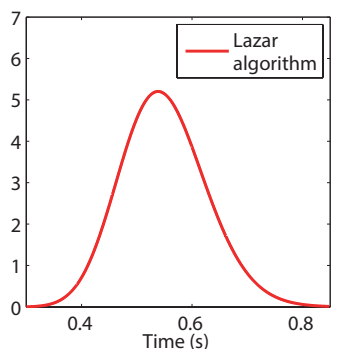

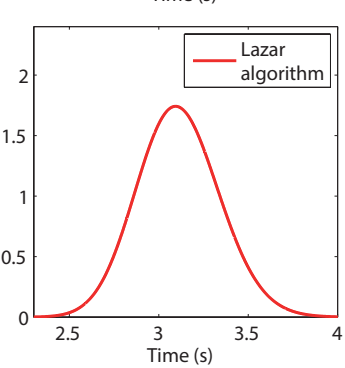

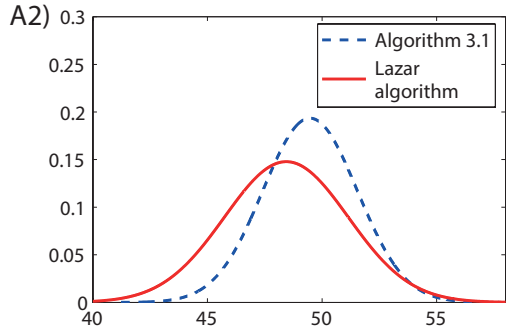

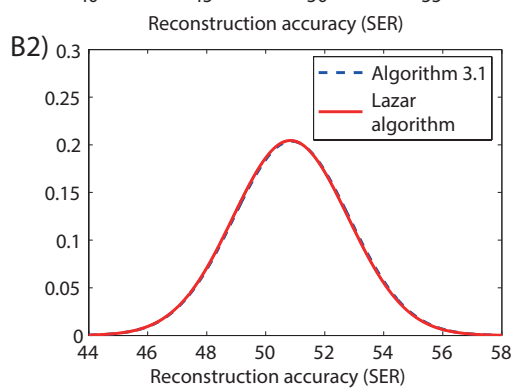

Figure 3.7: Comparison between Algorithm 3.1 and Lazar algorithm for 100 input sequences. Probability density functions for $10^{3}$ iterations corresponding to A1) reconstruction time and $\mathrm{A} 2$ ) reconstruction accuracy, and for $10^{4}$ iterations corresponding to $\mathrm{B} 1$ ) reconstruction time and $\mathrm{B} 2$ ) reconstruction accuracy .

one iteration.

\subsubsection{Numerical study for Algorithm 3.2}

In this subsection, Algorithm 3.2 is compared with the standard algorithm in (Lazar and Pnevmatikakis, 2008a).

To investigate numerically the relationship between $M$ and the reconstruction $S E R$, sequences $\left\{\mathcal{T}_{u^{j}}\right\}_{k=1, \ldots, 100}$ were used to reconstruct inputs $u^{j}, j=1, \ldots, 100$, for $M$ varying from 1 to 5 .

The inputs $\left\{u^{j}\right\}_{j=1,100}$ were reconstructed for each value of $M$ using Algorithm 3.2. Figure 3.9 displays the means and standard deviations of the SER values for each bandwidth $\bar{\Omega}_{M}=\frac{M \Omega}{b-c}$ of $\bar{\psi}_{M}{ }^{\prime}$. For $M \geq 2$ the $S E R$ saturates and, based on a large number of numerical simulations carried out, it was concluded that $M=2$ can be used to achieve good results.

The inputs $\left\{u^{j}\right\}_{j=1, \ldots, 100}$ were reconstructed from the spike-time sequences $\left\{\mathcal{T}_{u^{j}}\right\}_{j=1, \ldots, 100}$ using Algorithm 3.2 and the algorithm in (Lazar and Pnevmatikakis, 2008a).

The performance of the algorithms was assessed by computing the SER and measuring the reconstruction time. The results, depicted in Figure 3.10, show that the computing time for Algorithm 3.2 is more than two orders of magnitude faster than the standard 


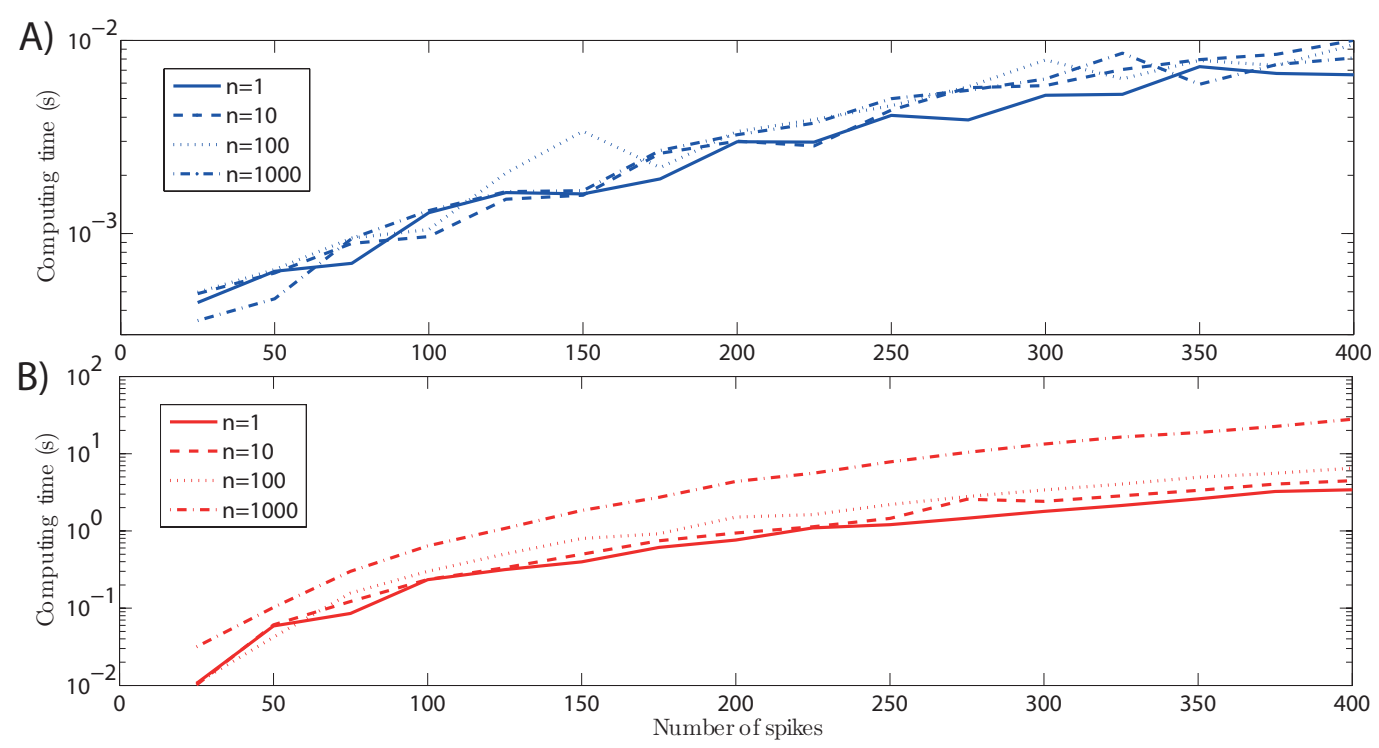

Figure 3.8: Computation time as a function of the number of spikes used in reconstruction for $n$ iterations: A) Algorithm 3.1 and B) the standard algorithm.

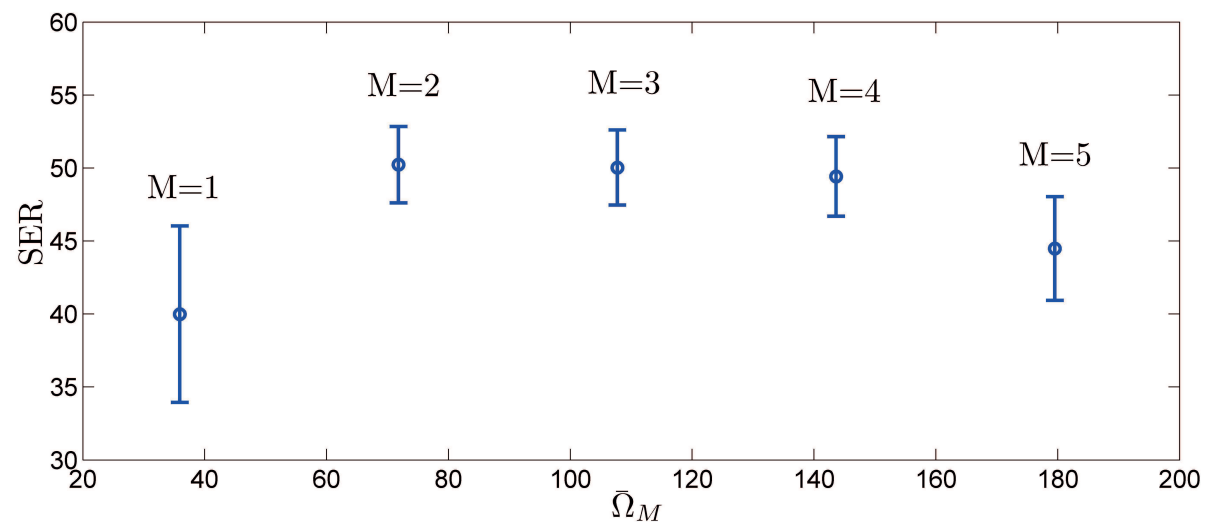

Figure 3.9: Reconstruction SER of Algorithm 3.2 for different values of $\bar{\Omega}_{M}$. 
method. Let $\left\{E^{j}(t)\right\}_{j=1,2,3}$ denote the normalised errors $E(t)$ corresponding to input functions $\left\{u^{j}\right\}_{j=1,2,3}$, respectively. The error functions $\left\{E^{j}(t)\right\}_{j=1,2,3}$ are displayed in Figure 3.11 for both methods.
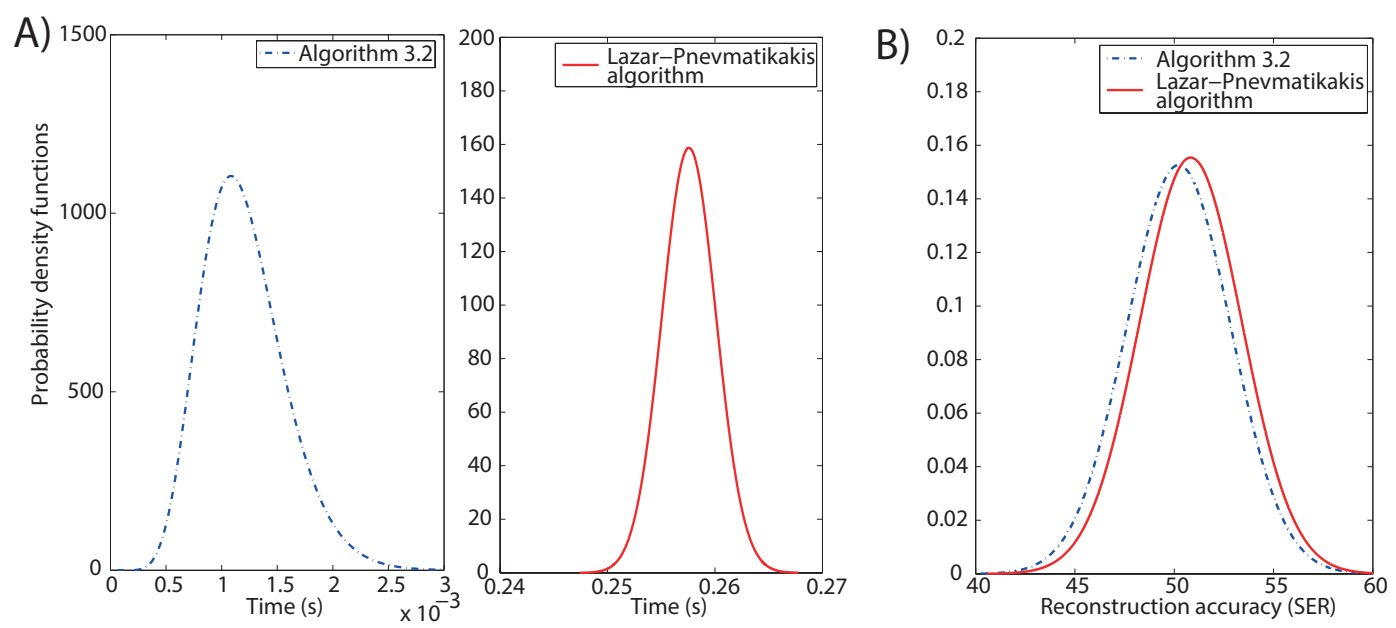

Figure 3.10: Comparison between Algorithm 3.2 and the Lazar-Pnevmatikakis algorithm. Probability density functions corresponding to A) reconstruction time and B) reconstruction accuracy, estimated based on 100 input sequences.

The input function $u^{1}(t)$ was then reconstructed from spike time sequences $\left\{t_{k}^{1}\right\}_{k=1, \ldots, N}$, with lengths $N=25,50, \ldots 400$, respectively, using both algorithms. The results, depicted in Figure 3.12, show that Algorithm 3.2 is far less sensitive to the length of processed the spike time sequences. Specifically, the computation time corresponding to $N=400$ spikes increases 23.51 times for the proposed algorithm and 236.24 times for the LazarPnevmatikakis algorithm, compared to the time taken to process 25 spikes.

\subsubsection{Error evaluation for the interpolation step of the proposed algorithms}

A piecewise linear interpolation scheme was used to implement step 7 of both proposed algorithms. The following errors are defined in order to evaluate separately the impact of different interpolation schemes on the overall error

$$
e(t)=\frac{1}{100} \sum_{j=1}^{100} e^{j}(t)
$$

where

$$
e^{j}(t)=\frac{\left|u^{j}(t)-\widetilde{u}^{j}(t)\right|}{\left\|u^{j}\right\|_{L^{2}}} \cdot 100
$$



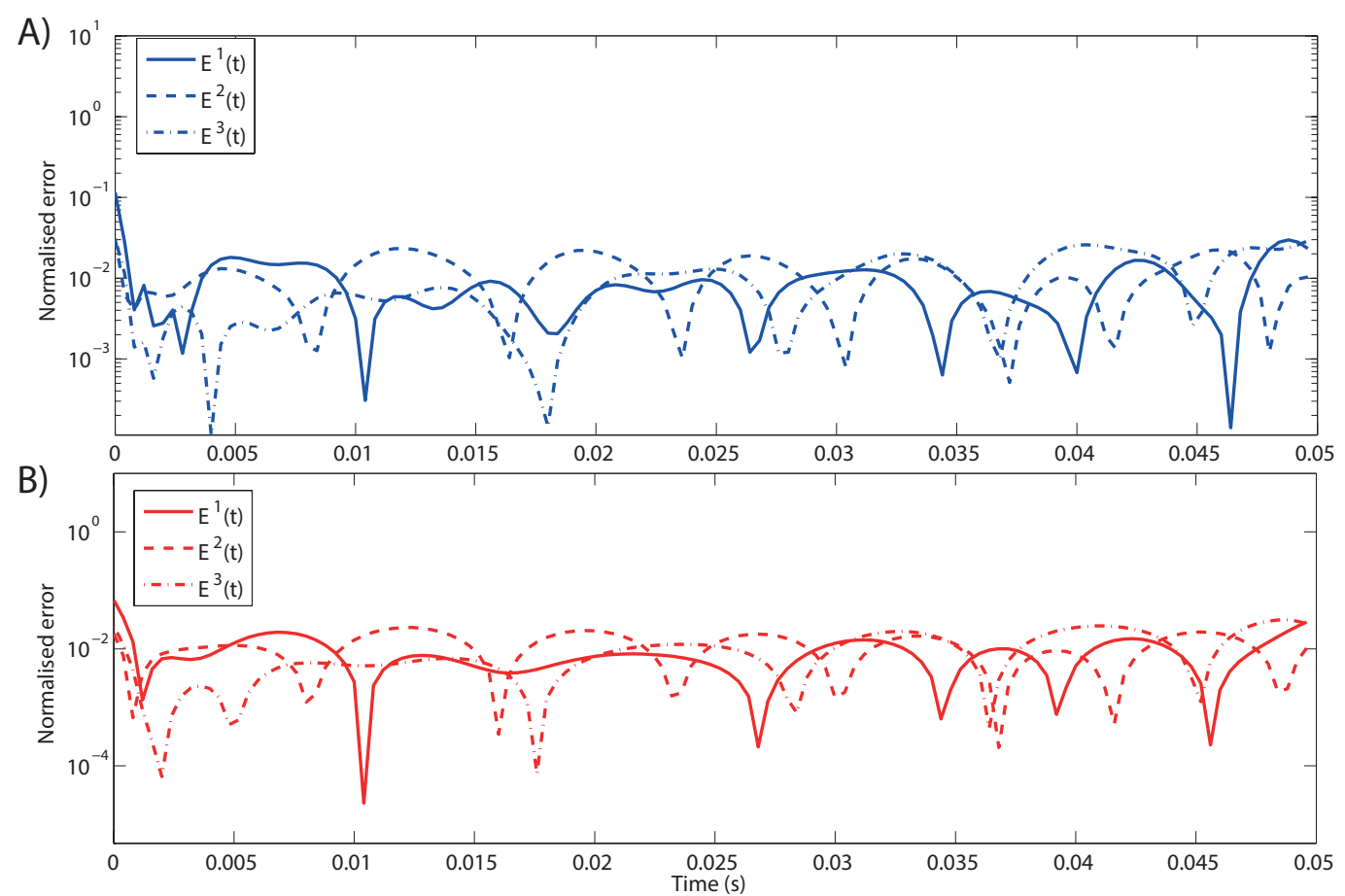

Figure 3.11: The normalised errors corresponding to input functions $\left\{u^{1}, u^{2}, u^{3}\right\}$ for A) Algorithm 3.2 and B) the Lazar-Pnevmatikakis algorithm. 


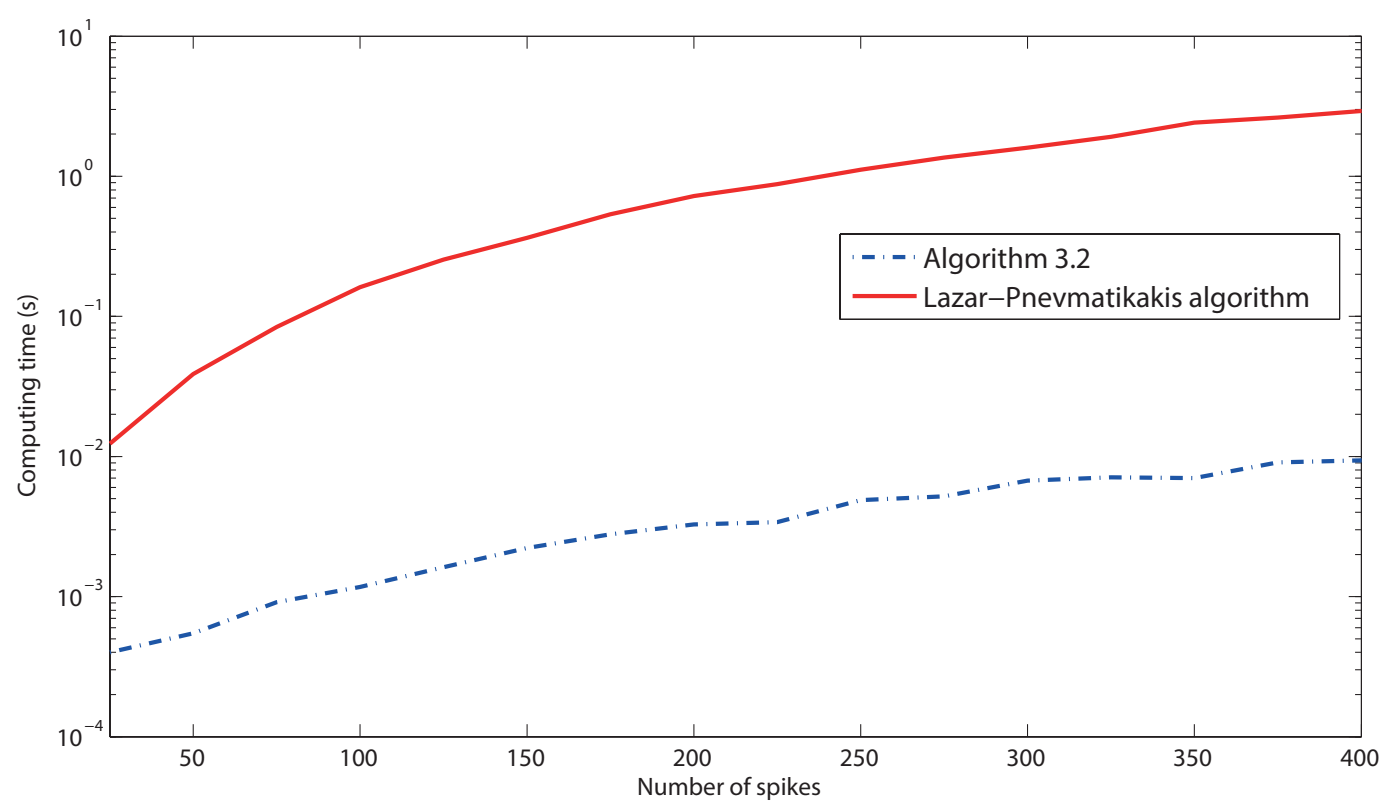

Figure 3.12: Computation time as function of the number of spikes used in reconstruction.

and $\widetilde{u}^{j}$ denotes the piecewise linear interpolation of $u^{j}$ at points $\left\{\psi_{\text {rec }}^{j}(l \varepsilon)\right\}_{l=0, \ldots, \bar{L}}$. Error $e(t)$ is depicted in Figure 3.13. Moreover, the following error is defined

$$
\bar{e}=\frac{1}{100} \sum_{j=1}^{100}\left\|e^{j}\right\|_{L^{2}} .
$$

The error in (3.33), representing the average error percentage out of $\left\|u^{j}\right\|_{L^{2}}$, was evaluated as $\bar{e}=6.7 \cdot 10^{-6} \%$.

Furthermore, the simulation was repeated several times, by changing the linear interpolation of $\left\{u^{j}\right\}_{j=1, \ldots, 100}$ to higher order spline interpolation, and noticed that $\bar{e}$ was not significantly different.

\subsection{Conclusions}

The classical formulation of the encoding mechanism of an IF neuron is based on the projection of the input onto a set of functions that depend both on the input and the nonuniform output time sequence.

This chapter formulated the IF encoding as a problem of uniform sampling on a set of input independent time points. Two novel reconstruction algorithms were proposed based 


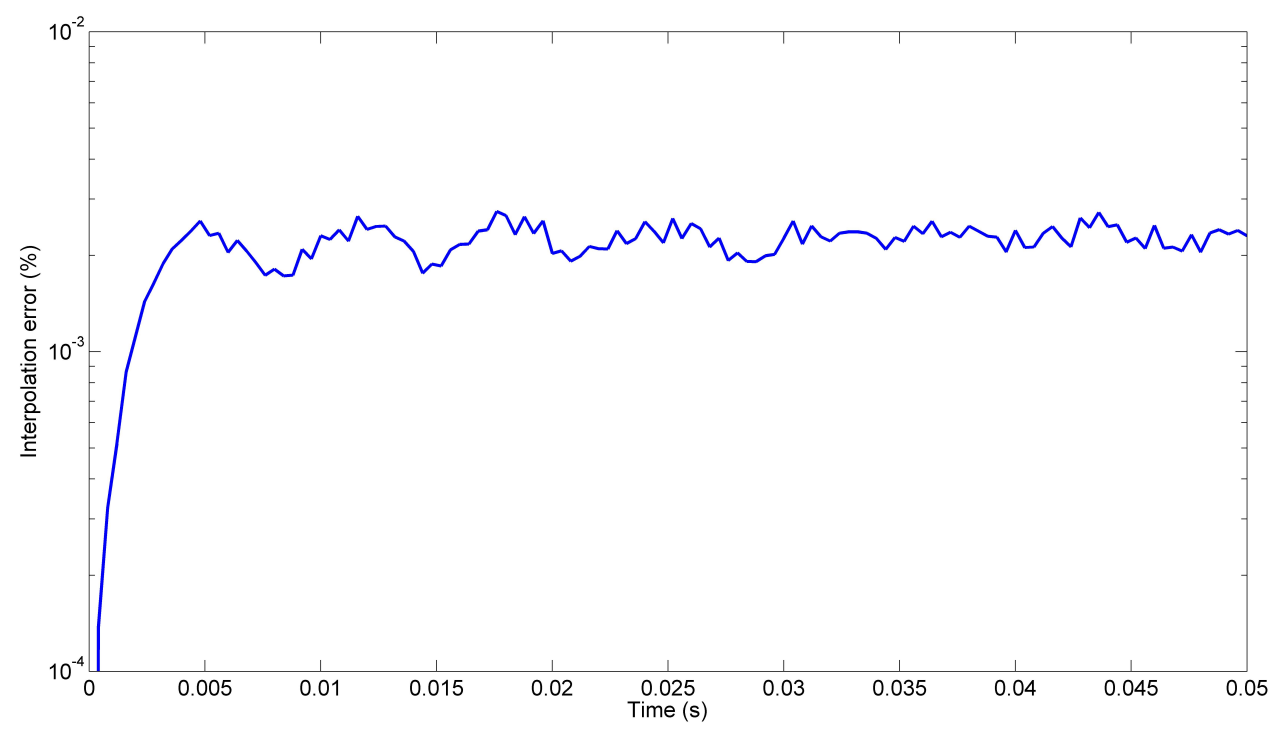

Figure 3.13: The average normalised error $e(t)$, corresponding to the linear interpolation of inputs $\left\{u^{j}(t)\right\}_{j=1, \ldots, 100}$.

on this new formulation. Numerical results were presented to show that the two proposed algorithms are as accurate as the standard iterative (Lazar, 2004) and non-iterative (Lazar and Pnevmatikakis, 2008a) reconstruction methods, respectively, but a significantly lower computational complexity. In addition, the rate of increase in computation time for Algorithm 3.2, relative to the input size (number of spikes-time processed), is one order of magnitude lower than the rate for the algorithm in (Lazar and Pnevmatikakis, 2008a). Furthermore, as opposed to Algorithm 3.1, the rate of increase in the computation time of the standard iterative method (Lazar, 2004) is very sensitive to the number of iterations used in reconstruction. 


\section{Chapter 4}

\section{A novel reconstruction framework in shift-invariant spaces for signals encoded with integrate-and-fire neurons}

The signals measured in practice have a finite time support and a spectrum vanishing for high frequency components. Therefore, modelling these functions as part of a shift-invariant space (SIS) generated by the sinc function, which has slow decay in time and rectangular spectrum, poses in many cases computational problems. These issues have been addressed by choosing a different generating function that is compactly supported, has a desired impulse response or has a smooth decay to 0 (Aldroubi and Gröchenig, 2001).

Gontier and Vetterli (2014) extended the results of Lazar and Tóth (2003) to SIS and developed an iterative method for reconstructing an input $u \in V^{2}(\lambda)$ of an integrate-andfire time encoding machine (IF-TEM), where $V^{2}(\lambda)$ denotes the SIS with integer shifts generated by function $\lambda$. The reconstruction method was reviewed in Chapter 2 .

This chapter introduces new theoretical results that form the basis for a new non-iterative reconstruction method, that is more accurate than the one in (Gontier and Vetterli, 2014). Both methods are generalised for the reconstruction of IF-TEM inputs $u \in V_{T}^{2}(\lambda)$, where $V_{T}^{2}(\lambda)$ denotes the SIS with generic shifts of length $T$. Detailed algorithms are given for the implementation of the two methods for the particular case of the ideal IF neuron.

The approaches above reconstruct function $u$ directly from the corresponding IF-TEM nonuniform output sequence $\mathcal{I} \mathcal{T}_{u}$. In essence, the two direct methods reconstruct the input in a space spanned by a frame consisting of functions that depend on $u$ (Gontier and Vetterli, 2014). This is a disadvantage when reconstructing a very large number of inputs $u^{j}$ from sequences $\mathcal{I} \mathcal{T}_{u^{j}}=\left\{t_{k}^{j}\right\}_{k=1, \ldots, N+1}$, as the frame has to be recalculated for each input signal. 
The new framework introduced in Chapter 3 reformulates the nonuniform sampling operation performed by the ideal IF neuron on input $u$ as an equivalent uniform sampling operation performed on an auxiliary function $\bar{\psi}^{\prime}$. This chapter extends this result and shows that the problem of encoding a function $u$ with an IF-TEM into nonuniform sequence $\mathcal{I T}_{u}$ can be reformulated as an equivalent IF-TEM encoding problem of $\bar{\psi}^{\prime}$ into uniform sequence $\{k \bar{\delta}\}$.

Based on the results above, two new indirect methods are introduced for reconstructing $u \in V_{T}^{2}(\lambda)$ from $\mathcal{I} \mathcal{T}_{u}$ via the auxiliary function $\bar{\psi}^{\prime}$. The methods reconstruct function $\bar{\psi}^{\prime}$ iteratively and non-iteratively, respectively. Detailed algorithms are presented for the implementation of each method for the particular case of the ideal IF neuron.

All the reconstruction algorithms in this chapter are developed under the assumption that function $\lambda$ satisfies $\lambda \in[-S T, S T], S>0$, which is required for the numerical implementation of the reproducing kernel. According to Hardy's uncertainty principle (Hardy, 1933), a function cannot be compactly supported both in time and frequency domain. Therefore the compact time support of the generating function is traded off for an infinite bandwidth. As a consequence, the reconstruction algorithms presented in the previous chapter are not a particular case of the ones developed here. From a practical standpoint, the methods presented in this chapter are, compared to the ones in Chapter 3, better suited for practical applications that allow the reconstruction of signals having a wider frequency support with restrictions on the duration of transmission.

The direct and indirect algorithms developed in this chapter have been evaluated through numerical simulations. The results show that the indirect methods have a similar level of accuracy but are significantly faster than the direct ones.

This chapter is structured as follows. Section 4.1 introduces a new non-iterative direct reconstruction method for spike trains generated with IF-TEMs. Section 4.2 presents two algorithms that implement the iterative and non-iterative direct methods for the particular case of the ideal IF neuron. Section 4.3 introduces the new framework for describing the sampling operation performed by an IF-TEM. Based on this framework, two new indirect reconstruction algorithms are developed for the ideal IF neuron. Section 4.4 presents numerical simulations results that compare the direct and indirect methods. 


\subsection{A new non-iterative method for reconstructing signals in shift- invariant spaces from spike trains generated with IF-TEMs}

Lemma 2.3 states that shift-invariant space $V^{2}(\lambda)$, defined in (2.20), is a RKHS and that $V^{2}(\lambda) \hookrightarrow C(\mathbb{R})$ provided that $\lambda \in W(2.25)$.

A formal definition of an IF-TEM for functions belonging to shift-invariant spaces was introduced in Definition 2.14. Gontier and Vetterli (2014) proposed an iterative method for reconstructing the IF-TEM input $u \in V^{2}(\lambda)$ from the associated output sequence $\mathcal{I} \mathcal{T}_{u}$ (see Theorem 2.10).

A new non-iterative method that is more accurate than the one in (Gontier and Vetterli, 2014) is proposed in the following, by introducing the additional assumption that sequence $\mathcal{I} \mathcal{T}_{u}$ is relatively separated (see Definition 2.2). The next lemma is a new result that forms the basis for the new approach.

Lemma 4.1. Let $\mathcal{I} \mathcal{T}_{u}=\left\{t_{k}\right\}_{k \in \mathbb{Z}}$ be the sequence generated by an IF-TEM given the input $u \in V^{2}(\lambda), \lambda \in W$. Let $\mathcal{Z}: V^{2}(\lambda) \rightarrow V^{2}(\lambda)$ be an operator defined by

$$
(\mathcal{Z} v)(t) \triangleq \sum_{k \in \mathbb{Z}} \mathcal{L}_{k}^{\mathcal{I} \mathcal{T}_{u}} v \cdot K\left(s_{k+1}, t\right), \forall v \in V^{2}(\lambda)
$$

where $K$ is the reproducing kernel of $V^{2}(\lambda), s_{k+1} \triangleq \frac{t_{k}+t_{k+1}}{2}$, and $\mathcal{L}_{k}^{\mathcal{I} \mathcal{T}_{u}}: V^{2}(\lambda) \rightarrow$ $\mathbb{R}, \mathcal{L}_{k}^{\mathcal{I} \mathcal{T}_{u}} v \triangleq \int_{t_{k}}^{t_{k+1}} v(\tau) d \tau$.

Then $\mathcal{Z}$ is a well defined and bounded operator provided that $\mathcal{I} \mathcal{T}_{u}$ is $\Delta$-dense, relatively separated and strictly increasing.

Proof. Sequences $\mathcal{I} \mathcal{T}_{u}$ and $\left\{s_{k}\right\}_{k \in \mathbb{Z}}$ are strictly increasing. The following holds

$$
\begin{aligned}
\left|t_{k}-t_{m}\right|>\zeta & \Leftrightarrow\left|t_{k+1}-t_{k}\right|>\zeta \Leftrightarrow\left|t_{k+1}-t_{k-1}\right|>2 \zeta \\
& \Leftrightarrow\left|\frac{t_{k}+t_{k+1}}{2}-\frac{t_{k-1}+t_{k}}{2}\right|=\left|s_{k+1}-s_{k}\right|>\zeta \\
& \Leftrightarrow\left|s_{k}-s_{m}\right|>\zeta, \forall k, m \in \mathbb{Z} .
\end{aligned}
$$

According to Aldroubi and Gröchenig (2001), if $\left\{s_{k}\right\}_{k \in \mathbb{Z}}$ is relatively separated, then $\left\{K\left(s_{k}, \cdot\right)\right\}_{k \in \mathbb{Z}}$ is a Bessel sequence for $V^{2}(\lambda)$. Christensen (2003) proved that the latter statement is equivalent to

$$
\left\|\mathcal{S}_{C}\right\|_{\mathcal{H}}^{2} \leq B\|c\|_{\ell^{2}}^{2}
$$

where $c$ is an arbitrary sequence $\left\{c_{k}\right\}_{k \in \mathbb{Z}} \in l^{2}(\mathbb{R})$ and $\mathcal{S}: l^{2}(\mathbb{R}) \rightarrow V^{2}(\lambda)$ is a well defined operator with expression $\mathcal{S}_{c}=\sum_{k \in \mathbb{Z}} c_{k} K\left(s_{k+1}, \cdot\right)$. Thus operator $\mathcal{Z}$ is well defined provided that $\left\{\mathcal{L}_{k}^{\mathcal{I} \mathcal{T}_{u} u}\right\}_{k \in \mathbb{Z}} \in l^{2}(\mathbb{R})$. 
The function $u$ is continuous due to Lemma 2.3 and, according to the mean value theorem for integrals, it follows that $\forall k \in \mathbb{Z}$, there exist $\xi_{k}, \chi_{k} \in\left[t_{k}, t_{k+1}\right]$ such that

$$
\begin{aligned}
& \mathcal{L}_{k}^{\mathcal{I} \mathcal{T} u} u=\left(t_{k+1}-t_{k}\right) u\left(\xi_{k}\right),
\end{aligned}
$$

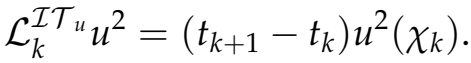

Using the Cauchy-Schwarz and AM-GM inequalities, it follows that

$$
\begin{aligned}
\sum_{k \in \mathbb{Z}}\left(\mathcal{L}_{k}^{\left.\mathcal{I} \mathcal{T}_{u} u\right)^{2}}\right. & =\left|\sum_{k \in \mathbb{Z}}\left(t_{k+1}-t_{k}\right)\left[\left(t_{k+1}-t_{k}\right) u^{2}\left(\chi_{k}\right)+\left(t_{k+1}-t_{k}\right)\left(u^{2}\left(\xi_{k}\right)-u^{2}\left(\chi_{k}\right)\right)\right]\right| \\
& \leq \Delta\left[\sum_{k \in \mathbb{Z}} \mathcal{L}_{k}^{\mathcal{I} \mathcal{T}_{u}} u^{2}+\Delta \sum_{k \in \mathbb{Z}}\left|\int_{\chi_{k}}^{\xi_{k}} 2 u(\tau) u^{\prime}(\tau) d \tau\right|\right] \\
& \leq \Delta\left[\|u\|_{L^{2}}^{2}+2 \Delta \sum_{k \in \mathbb{Z}}\left|\int_{\chi_{k}}^{\xi_{k}} u^{2}(\tau) d \tau\right|^{1 / 2} \cdot\left|\int_{\chi_{k}}^{\xi_{k}} u^{\prime 2}(\tau) d \tau\right|^{1 / 2}\right] \\
& \leq \Delta\left[\|u\|_{L^{2}}^{2}+\Delta \sum_{k \in \mathbb{Z}}\left|\int_{\chi_{k}}^{\xi_{k}} u^{2}(\tau) d \tau\right|+\Delta \sum_{k \in \mathbb{Z}}\left|\int_{\chi_{k}}^{\xi_{k}} u^{\prime 2}(\tau) d \tau\right|\right] \\
& \leq \Delta\left[\|u\|_{L^{2}}^{2}+\Delta\|u\|_{L^{2}}^{2}+\Delta\left\|u^{\prime}\right\|_{L^{2}}^{2}\right] .
\end{aligned}
$$

It follows that (2.24)

$$
\sum_{k \in \mathbb{Z}}\left(\mathcal{L}_{k}^{\mathcal{T}} u\right)^{2} \leq\|u\|_{L^{2}}^{2}\left(\Delta^{2}+\Delta^{2} / \rho^{2}+\Delta\right) .
$$

The following theorem presents a simplified formulation of the iterative reconstruction method from Theorem 2.10. Specifically, in the next proposed result, operator $\mathcal{P} \mathcal{Z}$ from Theorem 2.10 is substituted with operator $\mathcal{Z}$. This new result will form the basis of a new non-iterative reconstruction method.

Theorem 4.1. Let $\mathcal{I}_{u}=\left\{t_{k}\right\}_{k \in \mathbb{Z}}$ be the output sequence of an IF-TEM with test functions $\left\{\Phi_{k}\right\}_{k \in \mathbb{Z}}$ when presented with input $u \in V^{2}(\lambda), \lambda \in W$. Then $u$ can be reconstructed with arbitrary precision from $\mathcal{I} \mathcal{T}_{u}$, provided that $\mathcal{I T}_{u}$ is a $\Delta$-dense, relatively separated sequence and

$$
\Delta<\pi \rho,
$$

where $\rho=\inf _{\omega \in[0,2 \pi[} \frac{G_{\lambda}(\omega)}{G_{\lambda^{\prime}}(\omega)}$. The reconstruction is performed with

$$
\begin{gathered}
u_{0}=\mathcal{Z} u \\
u_{n+1}=u_{0}+(\mathcal{I}-\mathcal{Z}) u_{n} .
\end{gathered}
$$


The functions $u_{n}$ satisfy

$$
\left\|u-u_{n}\right\|_{L^{2}} \leq\left(\frac{\Delta}{\pi \rho}\right)^{n}\|u\|_{L^{2}}, \forall n \in \mathbb{N}
$$

Proof. According to Lemma 4.1, the following holds

$$
\mathcal{P}_{V^{2}} \mathcal{Z} u=\mathcal{Z} u, \forall u \in V^{2}(\lambda)
$$

where $\mathcal{P}_{V^{2}}: L^{2}(\mathbb{R}) \rightarrow V^{2}(\lambda)$ denotes the orthogonal projection operator onto $V^{2}(\lambda)$. Therefore the result follows from Theorem 2.10.

Theorem 4.1 is generalised in the following for inputs $u \in V_{T}^{2}(\lambda)$, where $V_{T}^{2}(\lambda)$ denotes the shift-invariant space with generic shifts of length $T>0$, defined by

$$
V_{T}^{2}(\lambda)=\left\{u(t)=\sum_{k \in \mathbb{Z}} c_{k} \lambda(t-k T),\left(c_{k}\right)_{k \in \mathbb{Z}} \in l^{2}(\mathbb{R})\right\} .
$$

Let $W_{T}$ be a space defined by

$$
W_{T}=\left\{\lambda \in H^{1}(\mathbb{R}): \exists A, B, B^{\prime}>0, A \leq \frac{G_{\lambda}^{T}(\omega)}{T} \leq B, G_{\lambda^{\prime}}^{T}(\omega) \leq B^{\prime}, \forall \omega \in[0,2 \pi]\right\},
$$

where

$$
G_{\lambda}^{T}(\omega)=\left(\sum_{k \in \mathbb{Z}}\left|\widehat{\lambda}\left(\frac{\omega+2 k \pi}{T}\right)\right|^{2}\right)^{1 / 2} .
$$

Furthermore, operator $\mathcal{Z}$ is extended to space $V_{T}^{2}(\lambda)$ such that $\mathcal{Z}: V_{T}^{2}(\lambda) \rightarrow V_{T}^{2}(\lambda)$, and

$$
(\mathcal{Z} v)(t) \triangleq \sum_{k \in \mathbb{Z}} \mathcal{L}_{k}^{\mathcal{I} \mathcal{T}_{u}} v \cdot K_{T}\left(s_{k+1}, t\right), \forall v \in V_{T}^{2}(\lambda)
$$

where $K_{T}=\frac{1}{T} K\left(\frac{\dot{T}}{T}, \dot{T}\right), K$ is the reproducing kernel of $V^{2}(\lambda), s_{k+1} \triangleq \frac{t_{k}+t_{k+1}}{2}$, and $\mathcal{L}_{k}^{\mathcal{I} \mathcal{T}_{u}}$ :

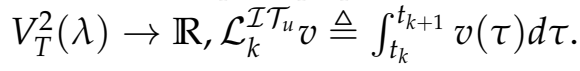

The following theorem is a new result for reconstructing an input $u \in V_{T}^{2}(\lambda)$ of an IF-TEM.

Theorem 4.2. Let $\mathcal{I} \mathcal{T}_{u}=\left\{t_{k}\right\}_{k \in \mathbb{Z}}$ be the output sequence of an IF-TEM with test functions $\left\{\Phi_{k}\right\}_{k \in \mathbb{Z}}$ given the input $u \in V_{T}^{2}(\lambda), \lambda \in W_{T}$. Then $u$ can be reconstructed with arbitrary precision from $\mathcal{I} \mathcal{T}_{u}$, provided that $\mathcal{I} \mathcal{T}_{u}$ is a $\Delta$-dense and relatively separated sequence and

$$
\Delta<\pi \rho_{T},
$$




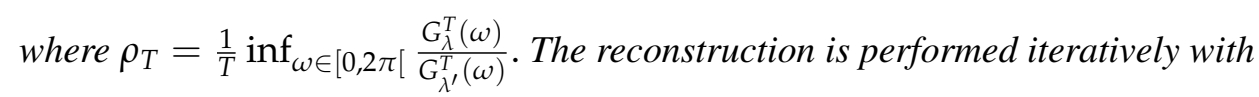

$$
\begin{gathered}
u_{0}=\mathcal{Z} u \\
u_{n+1}=u_{0}+(\mathcal{I}-\mathcal{Z}) u_{n}, n \geq 0,
\end{gathered}
$$

where $\mathcal{I}$ is the identity operator and the functions $u_{n}$ satisfy

$$
\left\|u-u_{n}\right\|_{L^{2}} \leq\left(\frac{\Delta}{\pi \rho_{T}}\right)^{n+1}\|u\|_{L^{2}}, \forall n \in \mathbb{N}^{*}
$$

Proof. Let $u \in V_{T}^{2}(\lambda)$. Then the following holds

$$
u(T t)=\sum_{k \in \mathbb{Z}} c_{k} \lambda(t T-k T)=\sum_{k \in \mathbb{Z}} c_{k} \lambda_{T}(t-k),
$$

where $\lambda_{T} \triangleq \lambda(T \cdot)$. It follows that $u_{T} \triangleq u(T \cdot) \in V^{2}\left(\lambda_{T}\right)$. Furthermore, the following holds

$$
\begin{aligned}
& \widehat{\lambda_{T}}(\omega)=\int_{\mathbb{R}} \lambda(T \tau) e^{-i \omega \tau} d \tau \stackrel{\tau=\tau_{1} / T}{=} \frac{1}{T} \int_{\mathbb{R}} \lambda\left(\tau_{1}\right) e^{-i \frac{\omega}{T} \tau_{1}} d \tau_{1}=\frac{1}{T} \widehat{\lambda}\left(\frac{\omega}{T}\right), \\
& \widehat{\lambda_{T}^{\prime}}(\omega)=T \int_{\mathbb{R}} \lambda^{\prime}(T \tau) e^{-i \omega \tau} d \tau \stackrel{\tau=\tau_{1} / T}{=} \int_{\mathbb{R}} \lambda^{\prime}\left(\tau_{1}\right) e^{-i \frac{\omega}{T} \tau_{1}} d \tau_{1}=\widehat{\lambda}^{\prime}\left(\frac{\omega}{T}\right) .
\end{aligned}
$$

The sequence $\mathcal{I} \mathcal{T}_{u}$ satisfies

$$
\Phi_{k}\left(t_{k+1}\right)=\int_{t_{k}}^{t_{k+1}} u(\tau) d \tau \stackrel{\tau=T \tau_{1}}{=} T \int_{t_{k} / T}^{t_{k+1} / T} u_{T}\left(\tau_{1}\right) d \tau_{1}=T \Phi_{k}^{T}\left(\frac{t_{k+1}}{T}\right),
$$

where $\Phi_{k}^{T}=\frac{\Phi_{k}(T \cdot)}{T}$. It follows that an IF-TEM with test functions $\left\{\Phi_{k}^{T}\right\}_{k \in \mathbb{Z}}$ generates the output sequence $\mathcal{I} \mathcal{T}_{u}^{T} \triangleq\left\{\frac{t_{k}}{T}\right\}_{k \in \mathbb{Z}}$ when presented with input $u_{T} \in V^{2}\left(\lambda_{T}\right)$.

The sequence $\mathcal{I} \mathcal{T}_{u}^{T}$ is $\frac{\Delta}{T}$ - dense and relatively separated. According to Theorem 4.1, $u_{T}$ can be reconstructed with arbitrary precision from $\mathcal{I} \mathcal{T}_{u}^{T}$ provided that

$$
\frac{\Delta}{T}<\pi \rho,
$$

where $\rho=\inf _{\omega \in[0,2 \pi[} \frac{G_{\lambda_{T}}(\omega)}{G_{\lambda_{T}^{\prime}}(\omega)}$. The following hold

$$
G_{\lambda_{T}}(\omega)=\left(\sum_{k \in \mathbb{Z}}\left|\widehat{\lambda_{T}}(\omega+2 k \pi)\right|^{2}\right)^{1 / 2}=\frac{1}{T}\left(\sum_{k \in \mathbb{Z}}\left|\widehat{\lambda}\left(\frac{\omega+2 k \pi}{T}\right)\right|^{2}\right)^{1 / 2}=\frac{1}{T} G_{\lambda}^{T}(\omega),
$$




$$
G_{\lambda_{T}^{\prime}}(\omega)=\left(\sum_{k \in \mathbb{Z}}\left|\widehat{\lambda_{T}^{\prime}}(\omega+2 k \pi)\right|^{2}\right)^{1 / 2}=\left(\sum_{k \in \mathbb{Z}}\left|\widehat{\lambda^{\prime}}\left(\frac{\omega+2 k \pi}{T}\right)\right|^{2}\right)^{1 / 2}=G_{\lambda^{\prime}}^{T}(\omega) .
$$

It follows that $\lambda \in W_{T} \Leftrightarrow \lambda_{T} \in W$. The requirement (4.6) is equivalent to

$$
\Delta<\pi \rho_{T}
$$

where $\rho_{T} \triangleq \inf _{\omega \in\left[0,2 \pi\left[\frac{G_{\lambda}^{T}(\omega)}{G_{\lambda^{\prime}}^{T}(\omega)}\right.\right.}$.

Provided that (4.7) holds and $\lambda \in W_{T}$, by applying Theorem 4.1 it follows that the function $u_{T}$ can be reconstructed from $\mathcal{I} \mathcal{T}_{u}^{T}$ as

$$
\begin{gathered}
u_{T, 0}=\mathcal{Z} u_{T} \\
u_{T, n+1}=u_{T, 0}+(\mathcal{I}-\mathcal{Z}) u_{T, n}, n \geq 0 .
\end{gathered}
$$

The functions $u_{T, n}$ satisfy

$$
\left\|u_{T}-u_{T, n}\right\|_{L^{2}} \leq\left(\frac{\Delta / T}{\pi \rho}\right)^{n}\left\|u_{T}\right\|_{L^{2}}, \forall n \in \mathbb{N}
$$

The following holds by substituting $u_{n}=u_{T, n}(\dot{\bar{T}}), \forall n \in \mathbb{N}$, and $u=u_{T}(\dot{\bar{T}})$ in (4.8) and (4.9)

$$
\begin{gathered}
u_{0}=\left(\mathcal{Z} u_{T}\right)(\dot{\bar{T}}) \\
u_{n+1}=u_{0}+u_{n}-\left(\mathcal{Z} u_{T, n}\right)(\dot{\bar{T}}), n \geq 0,
\end{gathered}
$$

The operator $\mathcal{Z}$ satisfies (4.5)

$$
\begin{aligned}
& \left(\mathcal{Z} u_{T, n}\right)\left(\frac{t}{T}\right)=\sum_{k \in \mathbb{Z}} \mathcal{L}_{k}^{\mathcal{I} \mathcal{T}^{T}{ }^{T} u_{T, n} \cdot K} \cdot\left(\frac{s_{n+1}}{T}, \frac{t}{T}\right) \\
& =\sum_{k \in \mathbb{Z}} \mathcal{L}_{k}^{\mathcal{I} \mathcal{T}^{u} u_{n} \cdot K_{T}\left(s_{n+1}, t\right),} \\
& =\left(\mathcal{Z} u_{n}\right)(t), \forall t \in \mathbb{R},
\end{aligned}
$$

where $K_{T}(x, t) \triangleq \frac{1}{T} K\left(\frac{x}{T}, \frac{t}{T}\right), \forall x, t \in \mathbb{R}$. Therefore equations (4.2) hold true. Inequalities (4.3) are proven as follows.

$$
\begin{aligned}
& \left\|u_{T}-u_{T, n}\right\|_{L^{2}}^{2}=\int_{\mathbb{R}}\left(u_{T}(\tau)-u_{T, n}(\tau)\right)^{2} d \tau \stackrel{\tau=\tau_{1} / T}{=} \frac{1}{T} \int_{\mathbb{R}}\left(u\left(\tau_{1}\right)-u_{n}\left(\tau_{1}\right)\right)^{2} d \tau_{1}, \\
& \left\|u_{T}\right\|_{L^{2}}^{2}=\int_{\mathbb{R}}\left(u_{T}(\tau)\right)^{2} d \tau \stackrel{\tau=\tau_{1} / T}{=} \frac{1}{T} \int_{\mathbb{R}}\left(u\left(\tau_{1}\right)\right)^{2} d \tau_{1} .
\end{aligned}
$$

Therefore, according to (4.9), the theorem holds true.

The function $u \in V_{T}^{2}(\lambda)$ can be reconstructed non-iteratively from $\mathcal{I} \mathcal{T}_{u}$ as shown in the following new result that generalises Corollary 2 from (Lazar and Tóth, 2004b) for inputs 
belonging to shift-invariant spaces.

Corollary 4.1. Let $\mathcal{I} \mathcal{T}_{u}=\left\{t_{k}\right\}_{k \in \mathbb{Z}}$ be the output sequence of an IF-TEM with test functions $\left\{\Phi_{k}\right\}_{k \in \mathbb{Z}}$ when presented with input $u \in V_{T}^{2}(\lambda), \lambda \in W_{T}$.

Provided that $\Delta<\pi \rho_{T}$, u can be reconstructed iteratively from $\mathcal{I T}_{u}$ as

$$
\begin{gathered}
\boldsymbol{M}_{0}=\boldsymbol{I} \boldsymbol{d}, \\
\boldsymbol{M}_{j}=\boldsymbol{I} \boldsymbol{d}+\boldsymbol{M}_{j-1}(\boldsymbol{I} \boldsymbol{d}-\boldsymbol{Q}), j=1, \ldots, n, \\
u_{n}=\boldsymbol{K}^{T} \boldsymbol{M}_{n} \boldsymbol{q},
\end{gathered}
$$

such that

$$
\left\|u-u_{n}\right\|_{L^{2}} \leq\left(\frac{\Delta}{\pi \rho_{T}}\right)^{n+1}\|u\|_{L^{2}}, \forall n \in \mathbb{N}
$$

where Id represents the identity matrix, $\rho_{T}=\frac{1}{T} \inf _{\omega \in[0,2 \pi[} \frac{G_{\lambda}^{T}(\omega)}{G_{\lambda^{\prime}}^{T}(\omega)},[K]_{m}=K_{T}\left(s_{m+1}, \cdot\right)$, $K_{T}=\frac{1}{T} K(\dot{\bar{T}}, \dot{\bar{T}}), K$ is the reproducing kernel of $V^{2}(\lambda(T \cdot)),[\boldsymbol{Q}]_{m, k}=\mathcal{L}_{m}^{\mathcal{I} \mathcal{T}_{u}}\left(K_{T}\left(s_{k+1}, \cdot\right)\right)$,

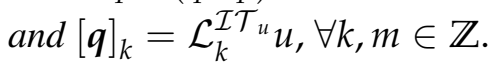

Under the same assumptions, $u$ can be reconstructed non-iteratively from $\mathcal{I} \mathcal{T}_{u}$ as

$$
u=K^{T} Q^{+} q
$$

Proof. According to Theorem 4.2, $\lim _{n \rightarrow \infty} u_{n}=u$ and

$$
u_{0}=\sum_{k \in \mathbb{Z}} \mathcal{L}_{k}^{\mathcal{T}} u \cdot K\left(s_{k+1}, \cdot\right)=\boldsymbol{K}^{T} \boldsymbol{q}
$$

Equations (4.10) are proven by induction as follows. Assuming that $u_{n}=\boldsymbol{K}^{T} \boldsymbol{M}_{n} \boldsymbol{q}$ holds, it follows that (4.2)

$$
u_{n+1}=u_{n}+\mathcal{Z}\left(u-u_{n}\right)=\boldsymbol{K}^{T} \boldsymbol{M}_{n} \boldsymbol{q}+\boldsymbol{K}^{T} \boldsymbol{q}-\mathcal{Z} u_{n} .
$$

The last term can be calculated with

$$
\mathcal{Z} u_{n}=\sum_{m \in \mathbb{Z}}\left(\sum_{k \in \mathbb{Z}}\left[\boldsymbol{M}_{n} \boldsymbol{q}\right]_{k} \cdot \mathcal{L}_{m}^{\mathcal{I} \mathcal{T}_{u}}\left(K_{T}\left(s_{k+1}, \cdot\right)\right)\right) K_{T}\left(s_{m+1}, \cdot\right)=\boldsymbol{K}^{T} \boldsymbol{Q} \boldsymbol{M}_{n} \boldsymbol{q} .
$$

From (4.12), (4.13) it follows that

$$
u_{n+1}=\boldsymbol{K}^{T}\left(\boldsymbol{I} \boldsymbol{d}+(\boldsymbol{I} \boldsymbol{d}-\boldsymbol{Q}) \boldsymbol{M}_{n}\right) \boldsymbol{q}=\boldsymbol{K}^{T} \boldsymbol{M}_{n+1} \boldsymbol{q} .
$$


Finally, the equality holds (Lazar and Tóth, 2004b, Strohmer, 1991)

$$
\lim _{n \rightarrow \infty} u_{n}=\lim _{n \rightarrow \infty} \boldsymbol{K}^{T} \boldsymbol{M}_{n} \boldsymbol{q}=\boldsymbol{K}^{T} \boldsymbol{Q}^{+} \boldsymbol{q}
$$

According to Corollary 4.1, the iterative equations (4.10) perform an approximate reconstruction of input $u$, for $\forall n \in \mathbb{N}$. However, an iterative method is preferred for the scenario in which the time allocated for reconstruction is limited (Strohmer, 1991).

The parameter $\rho_{T}$ needs to be calculated in order to evaluate the reconstruction requirement $\Delta<\pi \rho_{T}$ in Corollary 4.1 .

Moreover, in the function $K_{T}$ is generally unknown. The following result proposes a method to calculate both $\rho_{T}$ and $K_{T}$ for a space generated by a compactly supported function.

Corollary 4.2. Let $\lambda: \mathbb{R} \rightarrow \mathbb{R}$, such that $\operatorname{supp}(\lambda)=[-S T, S T]$. Then the following holds

$$
\rho_{T}=\frac{1}{T} \inf _{\omega \in[0,2 \pi[} \frac{G_{\lambda}^{T}(\omega)}{G_{\lambda^{\prime}}^{T}(\omega)}
$$

where

$$
\begin{aligned}
& \left(G_{\lambda}^{T}\right)^{2}(\omega)=T \sum_{k=-2 S}^{2 S}\langle\lambda, \lambda(\cdot+k T)\rangle_{L^{2}} \cdot e^{i k \omega}, \\
& \left(G_{\lambda^{\prime}}^{T}\right)^{2}(\omega)=T \sum_{k=-2 S}^{2 S}\left\langle\lambda^{\prime}, \lambda^{\prime}(\cdot+k T)\right\rangle_{L^{2}} \cdot e^{i k \omega} .
\end{aligned}
$$

Moreover, if $\lambda \in W_{T}$ then

$$
K_{T}(x, t)=T \sum_{j=r-S+1}^{r+S}\left[\sum_{p \in \mathbb{Z}} d_{-p} \lambda(x-(p+j) T)\right] \lambda(t-j T), t \in[r T,(r+1) T[,
$$

where $r \in \mathbb{Z}$ and $\left\{d_{p}\right\}_{p \in \mathbb{Z}}$ denote the Fourier coefficients of $\left(G_{\lambda}^{T}(\omega)\right)^{-2}$.

Proof. See Appendix B. 


\subsection{Direct reconstruction algorithms for inputs encoded with ideal IF neurons}

The ideal IF neuron is an IF-TEM with test functions $\Phi_{k}(t)=\bar{\delta}-b\left(t-t_{k}\right), \forall k \in \mathbb{Z}$, where $\bar{\delta} \triangleq C \delta$, and $\delta, C$, and $b$ are the threshold, integration constant, and bias, respectively. The ideal IF neuron generates sequence of spike times $\mathcal{I} \mathcal{T}_{u}=\mathcal{T}_{u}$ that satisfies (Lazar and Pnevmatikakis, 2008a)

$$
\mathcal{L}_{k}^{\mathcal{T}_{u}} u=q_{k}, \forall k \in \mathbb{Z},
$$

where $q_{k} \triangleq \bar{\delta}-b\left(t_{k+1}-t_{k}\right)=\Phi_{k}\left(t_{k+1}\right), \forall k \in \mathbb{Z}$.

A new result based on Corollary 4.1 is presented in the following.

Corollary 4.3. Let $\mathcal{T}_{u}=\left\{t_{k}\right\}_{k \in \mathbb{Z}}$ be the sequence of spike times generated by an ideal IF neuron, for a given function $u \in V_{T}^{2}(\lambda), \lambda \in W_{T},|u(t)| \leq c<b, \forall t \in \mathbb{R}$. Then $u$ can be reconstructed from $\mathcal{T}_{u}$ if

$$
\frac{\bar{\delta}}{b-c}<\pi \rho_{T}
$$

Proof. Sequence $\mathcal{T}_{u}$ satisfies $\mathcal{L}_{k}^{\mathcal{T}_{u}} u=\bar{\delta}-b\left(t_{k+1}-t_{k}\right), \forall k \in \mathbb{Z}$.

The function $u$ satisfies (4.14) and is continuous. According to the mean value theorem for integrals, the following holds

$$
u\left(\xi_{k}\right)\left(t_{k+1}-t_{k}\right)=\bar{\delta}-b\left(t_{k+1}-t_{k}\right), \xi_{k} \in\left[t_{k}, t_{k+1}\right], \forall k \in \mathbb{Z} .
$$

and

$$
\frac{\bar{\delta}}{b+c} \leq t_{k+1}-t_{k}=\frac{\bar{\delta}}{u\left(\xi_{k}\right)+b} \leq \frac{\bar{\delta}}{b-c}, \forall k \in \mathbb{Z}
$$

Then $\mathcal{T}_{u}$ is $\frac{\bar{\delta}}{b-c}$ - dense, relatively separated, and thus by applying Corollary 4.1 the required result follows.

It is interesting to note that Theorem 1 from (Lazar and Pnevmatikakis, 2008a) represents a particular case of Corollary 4.3 for $T=\frac{\pi}{\Omega}, \lambda=\frac{\sin (\Omega \cdot)}{\pi \cdot}$, and $u \in P W_{\Omega}$.

Two practical algorithms based on corollaries 4.1 and 4.2, respectively, are proposed for reconstructing input $u_{\text {rec }}$ from the finite sequence of spike times $\mathcal{T}_{u}=\left\{t_{k}\right\}_{k=1, \ldots, N+1}$, which is generated using the discrete-time approximation of the ideal IF neuron (3.32). Signal $u_{\text {rec }}$ is computed in time points $\{l \varepsilon\}_{l=0, \ldots, L}$ such that $L \varepsilon \leq t_{N+1}$, where $\varepsilon$ is the sampling period used in reconstruction. The function $\lambda$ is assumed to satisfy $\operatorname{supp}(\lambda) \subseteq[-S T, S T]$. 


\section{Algorithm 4.1.}

Step 1. Calculate $q$ using

$$
[\boldsymbol{q}]_{m}=\bar{\delta}-b\left(t_{m+1}-t_{m}\right), m=1, \ldots, N
$$

Step 2. Calculate $K_{T}\left(s_{m+1}, l \varepsilon\right), Q, l=0, \ldots, L$, where

$$
\begin{gathered}
K_{T}\left(s_{m+1}, l \varepsilon\right)=T \sum_{j=r-S+1}^{r+S}\left[\sum_{p \in \mathbb{Z}} d_{-p} \lambda\left(s_{m+1}-(p+j) T\right)\right] \lambda(l \varepsilon-j T), \\
{[Q]_{m, k}=\int_{t_{m}}^{t_{m+1}} K_{T}\left(s_{k+1}, \tau\right) d \tau, k, m=1, \ldots, N}
\end{gathered}
$$

where $r=\left\lceil\frac{l \varepsilon}{T}\right\rceil$ and $\left\{d_{p}\right\}_{k \in \mathbb{Z}}$ denote the Fourier coefficients of $\left(G_{\lambda}^{T}(\omega)\right)^{-2}$.

Step 3. Calculate $\boldsymbol{M}_{n}$ iteratively as

$$
\begin{gathered}
\boldsymbol{M}_{0}=\boldsymbol{I} ; \\
\boldsymbol{M}_{j}=\boldsymbol{I}+\boldsymbol{M}_{j-1}(\boldsymbol{I}-\boldsymbol{M}), j=1, \ldots, n ;
\end{gathered}
$$

Step 4. Calculate $c$ as

$$
c=M_{n} \boldsymbol{q}
$$

Step 5. Calculate $u_{r e c}(l \varepsilon), l=0, \ldots, L$, where

$$
u_{r e c}(l \varepsilon)=\sum_{m=1}^{N} c_{m} K_{T}\left(s_{m+1}, l \varepsilon\right)
$$

The following is satisfied according to Corollary 4.1

$$
\lim _{n \rightarrow \infty} \boldsymbol{M}_{n}=Q^{+}
$$

Based on this observation, a more accurate non-iterative algorithm is presented as follows. 


\section{Algorithm 4.2.}

Step 1. Calculate $q$

$$
[\boldsymbol{q}]_{m}=\bar{\delta}-b\left(t_{m+1}-t_{m}\right), m=1, \ldots, N
$$

Step 2. Calculate $K_{T}\left(s_{m+1}, l \varepsilon\right), Q, l=0, \ldots, L$, where

$$
\begin{gathered}
K_{T}\left(s_{m+1}, l \varepsilon\right)=T \sum_{j=r-S+1}^{r+S}\left[\sum_{p \in \mathbb{Z}} d_{-p} \lambda\left(s_{m+1}-(p+j) T\right)\right] \lambda(l \varepsilon-j T), \\
{[Q]_{m, k}=\int_{t_{m}}^{t_{m+1}} K_{T}\left(s_{k+1}, \tau\right) d \tau, k, m=1, \ldots, N ;}
\end{gathered}
$$

where $r=\left\lceil\frac{\varepsilon}{T}\right\rceil$ and $\left\{d_{p}\right\}_{k \in \mathbb{Z}}$ denote the Fourier coefficients of $\left(G_{\lambda}^{T}(\omega)\right)^{-2}$.

Step 3. Calculate $Q^{+}$;

Step 4. Calculate $c$

$$
c=Q^{+} \boldsymbol{q}
$$

Step 5. Calculate $u_{r e c}(l \varepsilon), l=0, \ldots, L$, where

$$
u_{r e c}(l \varepsilon)=\sum_{m=1}^{N} c_{m} K_{T}\left(s_{m+1}, l \varepsilon\right)
$$

In order to reconstruct a set of inputs $\left\{u^{j}\right\}_{j=1, \ldots, R}$ from the corresponding set of spike sequences $\left\{\mathcal{T}_{u^{j}}\right\}_{j=1, \ldots, R}$ a new set of reproducing kernels $\left\{K_{T}\left(s_{m+1}, \cdot\right)\right\}_{m=1, \ldots, N}$, matrix $Q$, and sequence $\left\{\boldsymbol{M}_{j}\right\}_{j=0, \ldots, n}$ or pseudoinverse $\boldsymbol{Q}^{+}$have to be calculated for every $j$. This process is computationally demanding for large values of $R$ and $N$.

\subsection{Fast indirect reconstruction algorithms for inputs encoded with ideal IF neurons}

According to Theorem 3.2, for any ideal IF neuron with parameters $\bar{\delta}=C \delta$ and $b$ and for any function $u \in L^{2}(\mathbb{R}) \cap C(\mathbb{R})$ satisfying $|u| \leq c<b$, there exists uniquely a function $\bar{\psi}^{\prime}$ satisfying

$$
\mathcal{L}_{k}^{\mathcal{D}} \bar{\psi}^{\prime}=\bar{q}_{k}, \forall k \in \mathbb{Z},
$$

where $\bar{\psi}(x)=\psi(x)-x / b, \psi$ is the inverse of $y(t)=\int_{0}^{t}(u(\tau)+b) d \tau, \mathcal{D}=\{k \bar{\delta}\}_{k \in \mathbb{Z}}$, and $\bar{q}_{k}=\left(t_{k+2}-t_{k+1}\right)-\bar{\delta} / b$.

The following lemma proves that an IF-TEM encodes function $\bar{\psi}^{\prime}$ into uniform sequence of samples $\mathcal{D}$. This result forms the basis for a new method for reconstructing 
function $\bar{\psi}^{\prime}$.

Lemma 4.2. Let $u \in L^{2}(\mathbb{R}) \cap C(\mathbb{R}),|u| \leq c<b$, and let $\bar{\psi}(x)=\psi(x)-x / b$, where $\psi$ is the inverse of $y(t)=\int_{0}^{t}(u(\tau)+b) d \tau$. Let $\bar{\Phi}_{k}: \mathbb{R} \rightarrow \mathbb{R}, \bar{\Phi}_{k}(x)=t_{k+2}-t_{k+1}-(x-$ $k \bar{\delta}) / b, \forall k \in \mathbb{Z}, \forall x \in \mathbb{R}$, where $\mathcal{T}_{u}=\left\{t_{k}\right\}_{k \in \mathbb{Z}}$ represents the output sequence of an ideal IF neuron when presented with input $u$.

Then an IF-TEM with test functions $\left\{\bar{\Phi}_{k}\right\}_{k \in \mathbb{Z}}$ generates output sequence $\mathcal{D}=\{k \bar{\delta}\}_{k \in \mathbb{Z}}$ when presented with input $\bar{\psi}^{\prime}$.

Proof. Equation 4.15 is equivalent to

$$
\int_{k \bar{\delta}}^{(k+1) \bar{\delta}} \bar{\psi}^{\prime}(\tau) d \tau=\bar{\Phi}_{k}((k+1) \bar{\delta}), \forall k \in \mathbb{Z}
$$

According to Definition 2.14, the lemma holds true if

$$
\left.\int_{k \bar{\delta}}^{x} \bar{\psi}^{\prime}(\tau) d \tau<\bar{\Phi}_{k}(x), x \in\right] k \bar{\delta},(k+1) \bar{\delta}[, \forall k \in \mathbb{Z},
$$

or, equivalently

$$
\left.\int_{k \bar{\delta}}^{x}\left(\bar{\psi}^{\prime}(\tau)+\frac{1}{b}\right) d \tau<t_{k+2}-t_{k+1}, x \in\right] k \bar{\delta},(k+1) \bar{\delta}[, \forall k \in \mathbb{Z} .
$$

The following holds due to Theorem 3.2

$$
\psi^{\prime}=\bar{\psi}^{\prime}+\frac{1}{b}>0
$$

Therefore $\int_{k \bar{\delta}}^{x} \psi^{\prime}(\tau) d \tau$ is strictly increasing and (4.17) follows from (4.16).

According to the orthogonal projection theorem, the following holds true

$$
\forall \bar{T}>0, \forall \bar{\lambda} \in W_{\bar{T}}, \bar{\psi}^{\prime}=\bar{\psi}_{0}^{\prime}+e,
$$

such that $\bar{\psi}_{0}^{\prime} \in V_{\bar{T}}^{2}(\bar{\lambda})$ and $e \in V_{\bar{T}}^{2}(\bar{\lambda})^{\perp}$, where $V_{\bar{T}}^{2}(\bar{\lambda})^{\perp}$ is the orthogonal subspace of space $V_{\bar{T}}^{2}(\bar{\lambda})$. Then $\|e\|_{L^{2}} \leq\left\|\bar{\psi}^{\prime}\right\|_{L^{2}}<\infty$ and therefore the energy of $e$ can be bounded. It has been shown that this bound can be made arbitrarily small for particular cases, e.g. bandlimited spaces (Lemma 3.3). As a consequence, function $\bar{\psi}^{\prime}$ is reconstructed in the space $V_{\bar{T}}^{2}(\bar{\lambda})$.

Corollary 4.4. Let $\bar{\psi}^{\prime} \in V_{\bar{T}}^{2}(\bar{\lambda}), \bar{\lambda} \in W_{\bar{T}}, \mathcal{D}=\{k \bar{\delta}\}_{k \in \mathbb{Z}}$, such that

$$
\bar{\delta}<\pi \bar{\rho}_{\bar{T}}
$$




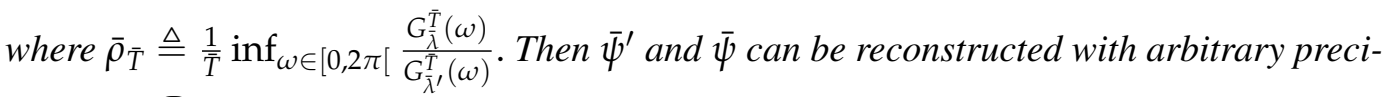
sion from $\mathcal{D}$ using

$$
\begin{gathered}
\overline{\boldsymbol{M}}_{0}=\boldsymbol{I} \boldsymbol{d}, \\
\overline{\boldsymbol{M}}_{j}=\boldsymbol{I} \boldsymbol{d}+\overline{\boldsymbol{M}}_{j-1}(\boldsymbol{I} \boldsymbol{d}-\overline{\boldsymbol{Q}}), j=1, \ldots, n, \\
\bar{\psi}_{n}^{\prime}=\overline{\boldsymbol{K}}_{1}^{*} \overline{\boldsymbol{M}}_{n} \overline{\boldsymbol{q}}, \\
\bar{\psi}_{n}=\overline{\boldsymbol{K}}_{2}^{*} \overline{\boldsymbol{M}}_{n} \overline{\boldsymbol{q}},
\end{gathered}
$$

such that

$$
\left\|\bar{\psi}^{\prime}-\bar{\psi}_{n}^{\prime}\right\|_{L^{2}} \leq\left(\frac{\bar{\delta}}{\pi \rho_{T}}\right)^{n+1}\left\|\bar{\psi}^{\prime}\right\|_{L^{2}}, \forall n \in \mathbb{N},
$$

where $[\overline{\boldsymbol{Q}}]_{m, k}=\mathcal{L}_{m}^{\mathcal{D}}\left(\bar{K}\left(\bar{s}_{k+1}, \cdot\right)\right),\left[\overline{\boldsymbol{K}}_{1}\right]_{m}=\bar{K}_{\bar{T}}\left(\bar{s}_{m+1}, \cdot\right),\left[\overline{\boldsymbol{K}}_{2}\right]_{m}=R_{\bar{s}_{m+1}}, R_{\bar{s}_{m+1}}(x)=$ $\int_{0}^{x} \bar{K}_{\bar{T}}\left(\bar{s}_{m+1}, \tau\right) d \tau, \forall x \in \mathbb{R}, \bar{K}_{\bar{T}}=\frac{1}{T} \bar{K}\left(\frac{\dot{\bar{T}}}{\bar{T}} \dot{\bar{T}}\right), \bar{K}$ is the reproducing kernel of $V^{2}(\bar{\lambda}(\bar{T} \cdot))$, $\bar{s}_{m}=\frac{2 m-1}{2} \bar{\delta}$, and $[\overline{\boldsymbol{q}}]_{k}=\left(t_{k+2}-t_{k+1}\right)-\bar{\delta} / b, \forall k, m \in \mathbb{Z}$.

Under the same assumptions, $\bar{\psi}^{\prime}$ and $\bar{\psi}$ can be reconstructed non-iteratively from $\mathcal{D}$ as

$$
\begin{aligned}
& \bar{\psi}^{\prime}=\overline{\boldsymbol{K}}_{1}^{*} \overline{\boldsymbol{Q}}^{+} \overline{\boldsymbol{q}}, \\
& \bar{\psi}=\overline{\boldsymbol{K}}_{2}^{*} \overline{\boldsymbol{Q}}^{+} \overline{\boldsymbol{q}},
\end{aligned}
$$

Proof. The result follows from Lemma 4.2 and Corollary 4.1.

The following holds according to Corollary 3.2

$$
u(\psi(x))=-\frac{b^{2} \bar{\psi}^{\prime}(x)}{b \bar{\psi}^{\prime}(x)+1}, \forall x \in \mathbb{R} .
$$

The values of $\{u(\psi(l \varepsilon))\}_{l=0, \ldots, \bar{L}}$ are calculated using (4.18), where $\psi(x)=\bar{\psi}(x)+$ $x / b, \forall x \in \mathbb{Z}$. The reconstructed signal is computed as the interpolation of $u$ at points $\{\psi(l \varepsilon)\}_{l=0, \ldots, \bar{L}}$. For piecewise linear interpolation, this amounts to calculating $\tilde{u}$, where

$$
\widetilde{u}(t)=u(\psi(l \varepsilon))+\frac{t-\psi(l \varepsilon)}{\psi((l+1) \varepsilon)-\psi(l \varepsilon)} \cdot[u(\psi((l+1) \varepsilon))-u(\psi(l \varepsilon))],
$$

for $\forall t \in[\psi(l \varepsilon), \psi((l+1) \varepsilon)[, l=0, \ldots, \bar{L}$.

A practical iterative reconstruction algorithm for function $u$ from finite sequence $\left\{t_{k}\right\}_{k=1, \ldots, N+1}$ based on Corollary 4.4 is summarised below. Function $\bar{\lambda}$ is assumed to satisfy $\operatorname{supp}(\bar{\lambda}) \subseteq$ $[-\bar{S} \bar{T}, \bar{S} \bar{T}]$. 


\section{Algorithm 4.3.}

Step 1. Calculate $\bar{K}_{\bar{T}}\left(\bar{s}_{m+1}, l \varepsilon\right), \bar{Q}_{,} R_{\bar{s}_{m+1}}(l \varepsilon), l=0, \ldots, \bar{L}$, where

$$
\begin{gathered}
\bar{K}_{\bar{T}}\left(s_{m+1}, l \varepsilon\right)=\bar{T} \sum_{j=r-\bar{S}+1}^{r+\bar{S}}\left[\sum_{p \in \mathbb{Z}} \bar{d}_{-p} \bar{\lambda}\left(s_{m+1}-(p+j) \bar{T}\right)\right] \bar{\lambda}(l \varepsilon-j \bar{T}), \\
{[\bar{Q}]_{k m}=\int_{k \delta}^{(k+1) \delta} \bar{K}_{\bar{T}}\left(\bar{s}_{m+1}, \tau\right) d \tau,} \\
R_{\bar{s}_{m+1}}(l \varepsilon)=\int_{0}^{l \varepsilon} \bar{K}_{\bar{T}}\left(\bar{s}_{m+1}, \tau\right) d \tau, k, m=1, \ldots, N ;
\end{gathered}
$$

where $r=\left\lceil\frac{l \varepsilon}{\bar{T}}\right\rceil$ and $\left\{\bar{d}_{p}\right\}_{k \in \mathbb{Z}}$ denote the Fourier coefficients of $\left(G_{\bar{\lambda}}^{\bar{T}}(\omega)\right)^{-2}$.

Step 2. Calculate $\bar{M}_{n}$ iteratively as

$$
\begin{gathered}
\overline{\mathbf{M}}_{0}=\mathbf{I} \boldsymbol{d}, \\
\overline{\boldsymbol{M}}_{j}=\boldsymbol{I} \boldsymbol{d}+\overline{\boldsymbol{M}}_{j-1}(\boldsymbol{I} \boldsymbol{d}-\overline{\mathbf{Q}}), j=1, \ldots, n
\end{gathered}
$$

Step 3. Calculate $\bar{q}$

$$
[\overline{\boldsymbol{q}}]_{m}=\left(t_{m+2}-t_{m+1}\right)-\bar{\delta} / b, m=1, \ldots, N
$$

Step 4. Calculate $\bar{c}$

$$
\overline{\boldsymbol{c}}=\overline{\boldsymbol{M}}_{n} \overline{\boldsymbol{q}}
$$

Step 5. Calculate $\bar{\psi}_{r e c}^{\prime}(l \varepsilon), \bar{\psi}_{r e c}(l \varepsilon)$, and $\psi_{r e c}(l \varepsilon), l=0, \ldots, \bar{L}$, where

$$
\begin{gathered}
\bar{\psi}_{r e c}^{\prime}(l \varepsilon)=\sum_{m=1}^{N} c_{m} \bar{K}_{\bar{T}}\left(\bar{s}_{m+1}, l \varepsilon\right), \\
\bar{\psi}_{r e c}(l \varepsilon)=\sum_{m=1}^{N} c_{m} R_{\bar{s}_{m+1}}(l \varepsilon), \\
\psi_{r e c}(l \varepsilon)=\bar{\psi}_{r e c}(l \varepsilon)+l \varepsilon / b ;
\end{gathered}
$$

Step 6. Calculate

$$
u_{r e c}\left(\psi_{r e c}(l \varepsilon)\right)=-\frac{b^{2} \bar{\psi}_{r e c}{ }^{\prime}(l \varepsilon)}{b \bar{\psi}_{r e c}(l \varepsilon)+1}, l=0, \ldots, \bar{L}
$$

Step 7. Calculate $\tilde{u}_{r e c}$, the interpolation of $u$ at points $\left\{\psi_{r e c}(l \varepsilon)\right\}_{l=0, \ldots, \bar{L}}$, and sample it at $\{j \varepsilon\}_{j=0, \ldots, L}$. For the particular case of piecewise linear interpolation

$$
\begin{aligned}
\widetilde{u}_{r e c}(j \varepsilon)= & u_{r e c}\left(\psi_{r e c}\left(l_{j} \varepsilon\right)\right)+\frac{j \varepsilon-\psi_{r e c}\left(l_{j} \varepsilon\right)}{\psi_{r e c}\left(\left(l_{j}+1\right) \varepsilon\right)-\psi_{r e c}\left(l_{j} \varepsilon\right)} \\
& \cdot\left[u_{r e c}\left(\psi_{r e c}\left(\left(l_{j}+1\right) \varepsilon\right)\right)-u_{r e c}\left(\psi_{r e c}\left(l_{j} \varepsilon\right)\right)\right],
\end{aligned}
$$

for $j=0, \ldots, L$, where $l_{j} \in \mathbb{Z}$ satisfies $\psi_{\text {rec }}\left(l_{j} \varepsilon\right) \leq j \varepsilon<\psi_{\text {rec }}\left(\left(l_{j}+1\right) \varepsilon\right)$. 
The following is satisfied according to Corollary 4.4

$$
\lim _{n \rightarrow \infty} \bar{M}_{n}=\bar{Q}^{+} .
$$

Based on this observation, a more accurate non-iterative algorithm is presented as follows. 


\section{Algorithm 4.4.}

Step 1. Calculate $\bar{K}_{\bar{T}}\left(\bar{s}_{m+1}, l \varepsilon\right), \bar{Q}_{,} R_{\bar{s}_{m+1}}(l \varepsilon), l=0, \ldots, \bar{L}$, where

$$
\begin{gathered}
\bar{K}_{\bar{T}}\left(s_{m+1}, l \varepsilon\right)=\bar{T} \sum_{j=r-\bar{S}+1}^{r+\bar{S}}\left[\sum_{p \in \mathbb{Z}} \bar{d}_{-p} \bar{\lambda}\left(s_{m+1}-(p+j) \bar{T}\right)\right] \bar{\lambda}(l \varepsilon-j \bar{T}), \\
{[\bar{Q}]_{k m}=\int_{k \delta}^{(k+1) \delta} \bar{K}_{\bar{T}}\left(\bar{s}_{m+1}, \tau\right) d \tau} \\
R_{\bar{s}_{m+1}}(l \varepsilon)=\int_{0}^{l \varepsilon} \bar{K}_{\bar{T}}\left(\bar{s}_{m+1}, \tau\right) d \tau, k, m=1, \ldots, N
\end{gathered}
$$

where $r=\left\lceil\frac{l_{\varepsilon}}{\bar{T}}\right\rceil$ and $\left\{\bar{d}_{p}\right\}_{k \in \mathbb{Z}}$ denote the Fourier coefficients of $\left(G_{\bar{\lambda}}^{\bar{T}}(\omega)\right)^{-2}$.

Step 2. Calculate $\bar{Q}^{+}$, the Moore-Penrose pseudoinverse of $\bar{Q}$.

Step 3. Calculate $\bar{q}$

$$
[\overline{\boldsymbol{q}}]_{m}=\left(t_{m+2}-t_{m+1}\right)-\bar{\delta} / b, m=1, \ldots, N
$$

Step 4. Calculate $\bar{c}$

$$
\bar{c}=\bar{Q}^{+} \bar{q}
$$

Step 5. Calculate $\bar{\psi}_{r e c}^{\prime}(l \varepsilon), \bar{\psi}_{r e c}(l \varepsilon)$, and $\psi_{r e c}(l \varepsilon), l=0, \ldots, \bar{L}$, where

$$
\begin{gathered}
\bar{\psi}_{r e c}^{\prime}(l \varepsilon)=\sum_{m=1}^{N} c_{m} \bar{K}_{\bar{T}}\left(\bar{s}_{m+1}, l \varepsilon\right), \\
\bar{\psi}_{r e c}(l \varepsilon)=\sum_{m=1}^{N} c_{m} R_{\bar{s}_{m+1}}(l \varepsilon), \\
\psi_{r e c}(l \varepsilon)=\bar{\psi}_{r e c}(l \varepsilon)+l \varepsilon / b
\end{gathered}
$$

Step 6. Calculate

$$
u_{r e c}\left(\psi_{r e c}(l \varepsilon)\right)=-\frac{b^{2} \bar{\psi}_{r e c}{ }^{\prime}(l \varepsilon)}{b \bar{\psi}_{r e c}{ }^{\prime}(l \varepsilon)+1}, l=0, \ldots, \bar{L}
$$

Step 7. Calculate $\tilde{u}_{r e c}$, the interpolation of $u$ at points $\left\{\psi_{r e c}(l \varepsilon)\right\}_{l=0, \ldots, \bar{L}}$, and sample it at $\{j \varepsilon\}_{j=0, \ldots, L}$. For the particular case of piecewise linear interpolation

$$
\begin{aligned}
\widetilde{u}_{r e c}(j \varepsilon)= & u_{r e c}\left(\psi_{r e c}\left(l_{j} \varepsilon\right)\right)+\frac{j \varepsilon-\psi_{r e c}\left(l_{j} \varepsilon\right)}{\psi_{r e c}\left(\left(l_{j}+1\right) \varepsilon\right)-\psi_{r e c}\left(l_{j} \varepsilon\right)} \\
& \cdot\left[u_{r e c}\left(\psi_{r e c}\left(\left(l_{j}+1\right) \varepsilon\right)\right)-u_{r e c}\left(\psi_{r e c}\left(l_{j} \varepsilon\right)\right)\right],
\end{aligned}
$$

for $j=0, \ldots, L$, where $l_{j} \in \mathbb{Z}$ satisfies $\psi_{r e c}\left(l_{j} \varepsilon\right) \leq j \varepsilon<\psi_{r e c}\left(\left(l_{j}+1\right) \varepsilon\right)$.

The algorithms above calculate matrices $\overline{\boldsymbol{Q}}^{+}, \boldsymbol{M}_{n}, \overline{\mathbf{K}}_{1}, \overline{\mathbf{K}}_{2}$ off-line, in steps 1 and 2. Processing subsequent sets of spike times for every new set $j$ of spikes $\mathcal{T}_{u^{j}}=\left\{t_{k}^{j}\right\}_{k \in \mathbb{Z}}$ involves 
only a few additions and multiplications to recover input $u^{j}$ on time interval $\left[t_{1}^{j}, t_{N+1}^{j}\right]$ (steps 3-7). Moreover, Step 2 of Algorithm 4.3, which performs the iterations, is computed off-line and thus the computation time is not dependent on the number of iterations.

\subsection{Numerical study}

In this section the computation time and reconstruction accuracy are compared through numerical simulations for the algorithms presented in this chapter. The accuracy is measured as signal-to-error $(S E R)$ ratio

$$
S E R=10 \log _{10}\left(\frac{\|u\|_{L^{2}}}{\left\|u-u_{\text {rec }}\right\|_{L^{2}}}\right)
$$

where $u$ and $u_{\text {rec }}$ denote the original and reconstructed signals, respectively. The computation time was evaluated only for the routine performing the reconstruction.

The algorithms were simulated for inputs $u \in V_{T}^{2}\left(\beta_{T}^{3}\right)$, where $\beta_{T}^{3}=\beta^{3}(\dot{\bar{T}})$. The function $\beta^{3}$ denotes the B-spline of degree 3 defined by (Unser, 1999)

$$
\beta^{3}(t)= \begin{cases}\frac{2}{3}-t^{2}+\frac{|t|^{3}}{2}, & 0 \leq|t|<1 \\ \frac{(2-|t|)^{3}}{6}, & 1 \leq|t|<2 \\ 0, & |t| \geq 2 .\end{cases}
$$

A set of 100 inputs $\left\{u^{j}\right\}_{j=1, \ldots, 100}$ were generated, where

$$
u^{j}(t)=\sum_{k=1}^{20} a_{j}^{k} \beta_{T}^{3}(t-k T), j=1, \ldots, 100, t \in[0,10],
$$

where $T=0.5$ and $a_{j}^{k}, k=1, \ldots, 20$, are random coefficients drawn from the standard uniform distribution on ]0,1[. Signals $u^{j}$ were sampled with period $\varepsilon=5 \cdot 10^{-2}$, and normalized such that $\max _{t \in[0,10]}|u(t)|=c=1$. Inputs $\left\{u^{j}\right\}_{j=1,2,3}$ are depicted in Figure 4.1.

An ideal IF neuron with parameters $\delta=1.2, C=1$, and $b=15$ generated spike time sequences $\left\{\mathcal{T}_{u^{j}}\right\}_{j}=1, \ldots, 100$ when presented with inputs $\left\{u^{j}(t)\right\}_{j=1, \ldots, 100}$. The encoding was performed using the following discrete-time approximation of the IF neuron.

$$
t_{k}^{j}=(l+1) \varepsilon-\varepsilon \cdot \frac{y^{j}((l+1) \varepsilon)-\bar{\delta}}{y^{j}((l+1) \varepsilon)-y^{j}(l \varepsilon)}, j, k=1, \ldots, 100,
$$

where $\bar{\delta}=C \delta, y^{j}(l \varepsilon)=\int_{0}^{l \varepsilon}\left(u^{j}(\tau)+b\right) d \tau$ is computed using the trapezoid rule, and $l$ is the unique solution of

$$
y^{j}(l \varepsilon) \leq k \bar{\delta}<y^{j}((l+1) \varepsilon)
$$




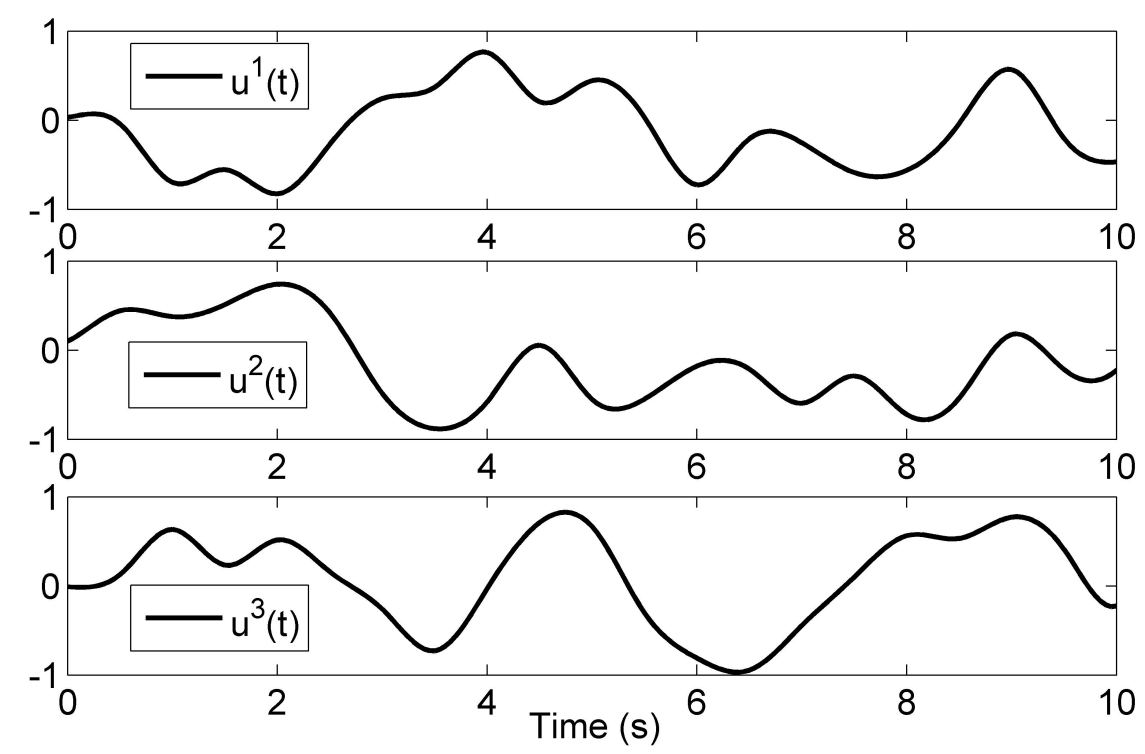

Figure 4.1: Input functions $\left\{u^{j}(t)\right\}_{j=1,2,3}$.

The value $\rho_{T}$ was calculated as shown in Corollary 4.2 for $S=2$ as $\rho_{T}=0.16$. The chosen parameters satisfy the condition $\frac{\bar{\delta}}{b-c}<\pi \rho_{T}$, which is required by Corollary 4.3. Based on a large number of simulations carried out, it was concluded that for $\bar{T}=4$ the proposed method in Corollary 4.4 achieves satisfactory results. Moreover, $\rho_{\bar{T}}$ was evaluated as $\rho_{\bar{T}}=1.27$ and condition $\bar{\delta}<\pi \rho_{\bar{T}}$ is also satisfied, as required in Corollary 4.4.

\subsubsection{Comparative numerical study of the iterative algorithms}

In order to investigate how the reconstruction $S E R$ and computing time are influenced by the number of iterations, sequences $\left\{\mathcal{T}_{u^{j}}\right\}_{j=1, \ldots, 100}$ were reconstructed with algorithms 4.1 and 4.3 for an increasing number of up to $n=250$ iterations. The average SER and computing time, calculated for each number of iterations for both algorithms, is depicted in Figure 4.2.

The results show that the accuracies of the two algorithms are not significantly different. However, the computation time for Algorithm 4.3 is from 1 to 2 orders of magnitude shorter than for Algorithm 4.1, and, unlike the latter, is not dependent on the number of iterations.

In order to investigate how the number of spikes used in reconstruction affects the computation time of both methods, sequences $\left\{t_{k}^{1}\right\}_{k=1, \ldots, P}$ with lengths $P=25,50, \ldots, 400$ was reconstructed with algorithms 4.1 and 4.3. The computation time of both algorithms is depicted for each number of spikes in Figure 4.3.

The results show that Algorithm 4.1 is more sensitive to the number of spikes used in reconstruction. Moreover, the computing time of Algorithm 4.3 is significantly less sensi- 


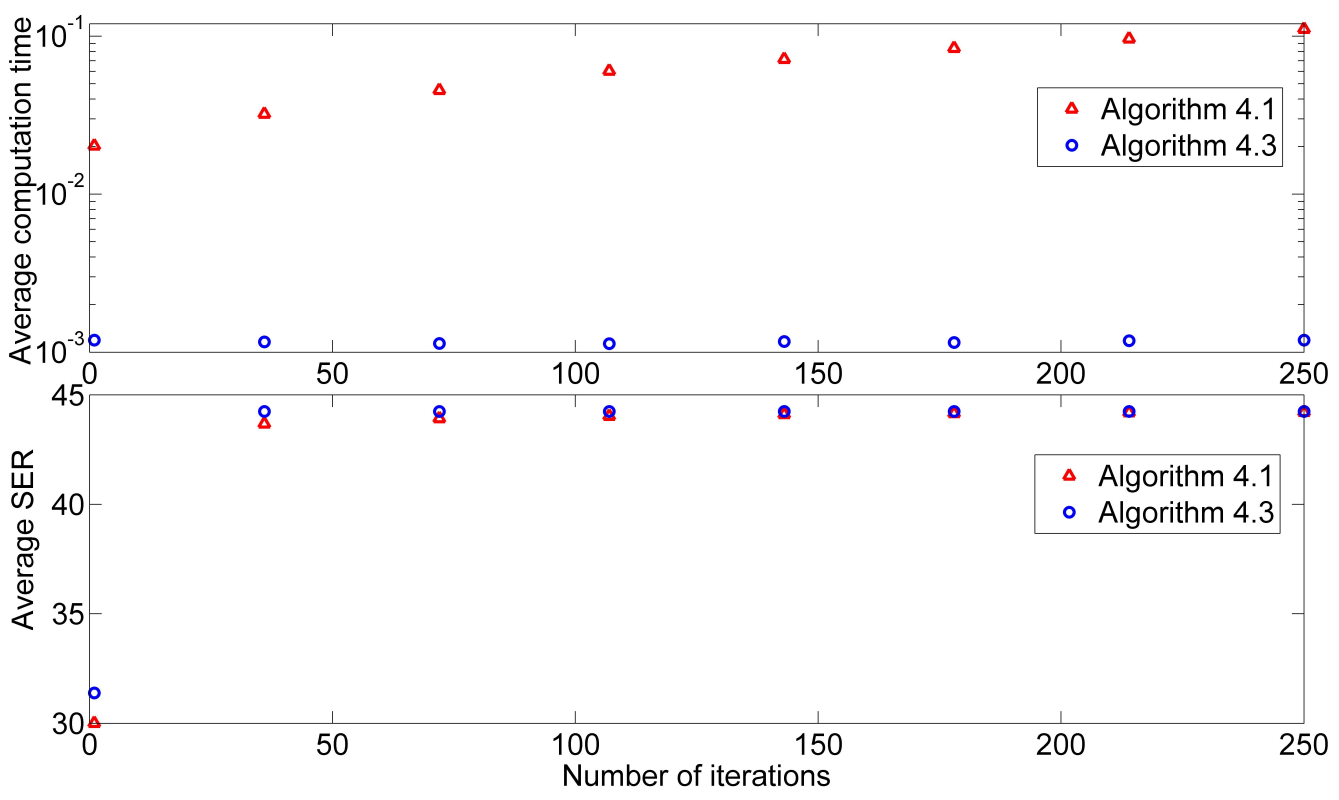

Figure 4.2: Average computation time (top) and SER (bottom) for 100 inputs reconstructed with algorithms 4.1 and 4.3 .

tive to the number of computed iterations. Specifically, for $n=1$, the computation time corresponding to 400 spikes increases 96 times for Algorithm 4.1 and 37 times for Algorithm 4.3. For $n=200$ iterations, the computation time corresponding to the same length of the spike sequence increases 738 and 24 times for Algorithm 4.1 and Algorithm 4.3, respectively.

\subsubsection{Comparative numerical study of the non-iterative algorithms}

The sequences $\left\{\mathcal{T}_{u^{j}}\right\}_{j=1, \ldots, 100}$ were reconstructed using algorithms 4.2 and 4.4, respectively. The normal probability density functions corresponding to the computing time and the SER are depicted in Figure 4.4. The results show that the SER is not significantly different for the two methods. However, the computing time for Algorithm 4.4 is more than one order of magnitude shorter than for Algorithm 4.2.

The sequences $\left\{t_{k}^{1}\right\}_{k=1, \ldots, P}$ were then reconstructed with Algorithm 4.2 and Algorithm 4.4 for $P=25,50, \ldots, 400$. The computation time of both algorithms is depicted in Figure 4.5 for each value of $P$. The results show that Algorithm 4.4 is far less sensitive to the length of the processed spike time sequence. Specifically, the computing time corresponding to $P=400$ increases 2 times for Algorithm 4.4 and 91 times for Algorithm 4.2, compared to the time taken to process 25 spikes. 

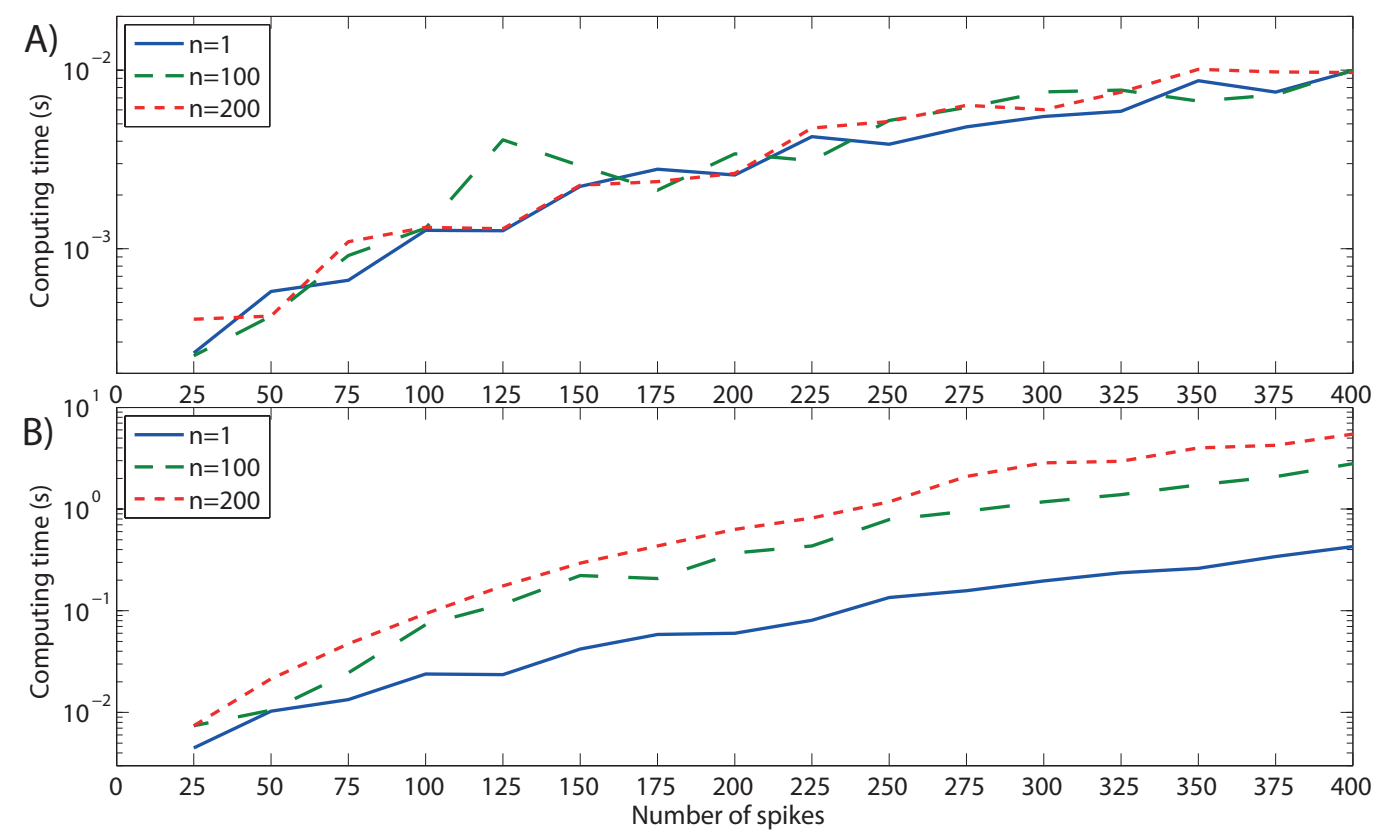

Figure 4.3: Computation time as a function of the number of spikes used in reconstruction for $n$ iterations: A) Algorithm 4.3 and B) Algorithm 4.1.
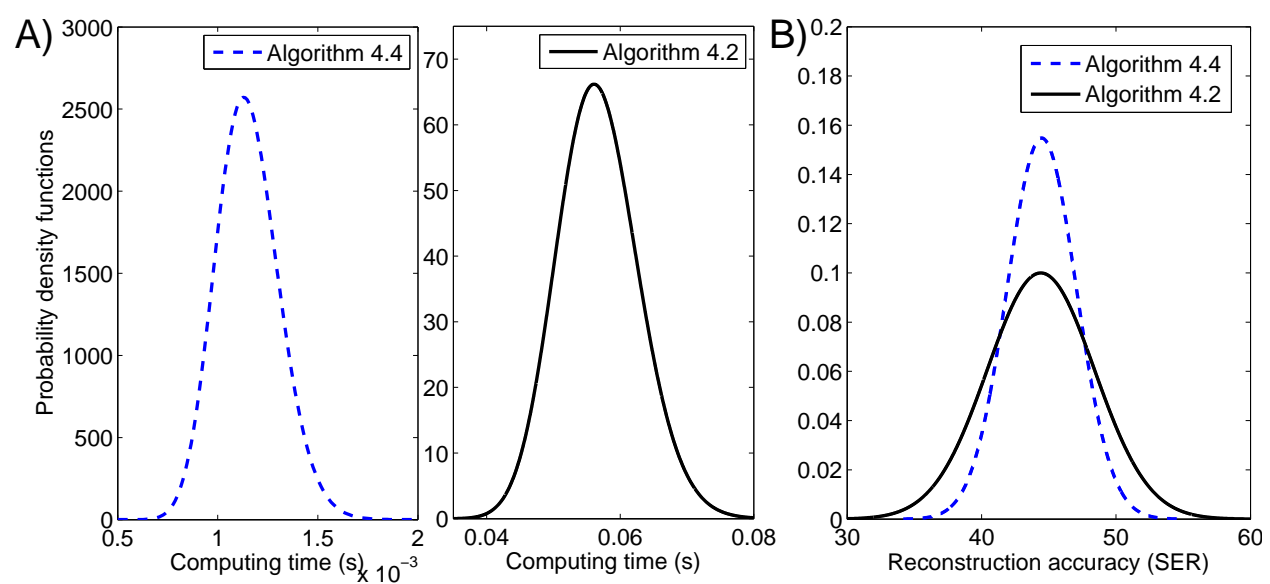

Figure 4.4: Comparison between Algorithm 4.2 and Algorithm 4.4. Probability density functions corresponding to: A) reconstruction time and B) reconstruction accuracy.

\subsection{Conclusions}

The IF-TEM is represented in the literature as a nonuniform sampling device whose time locations are input dependent (Gontier and Vetterli, 2014). 


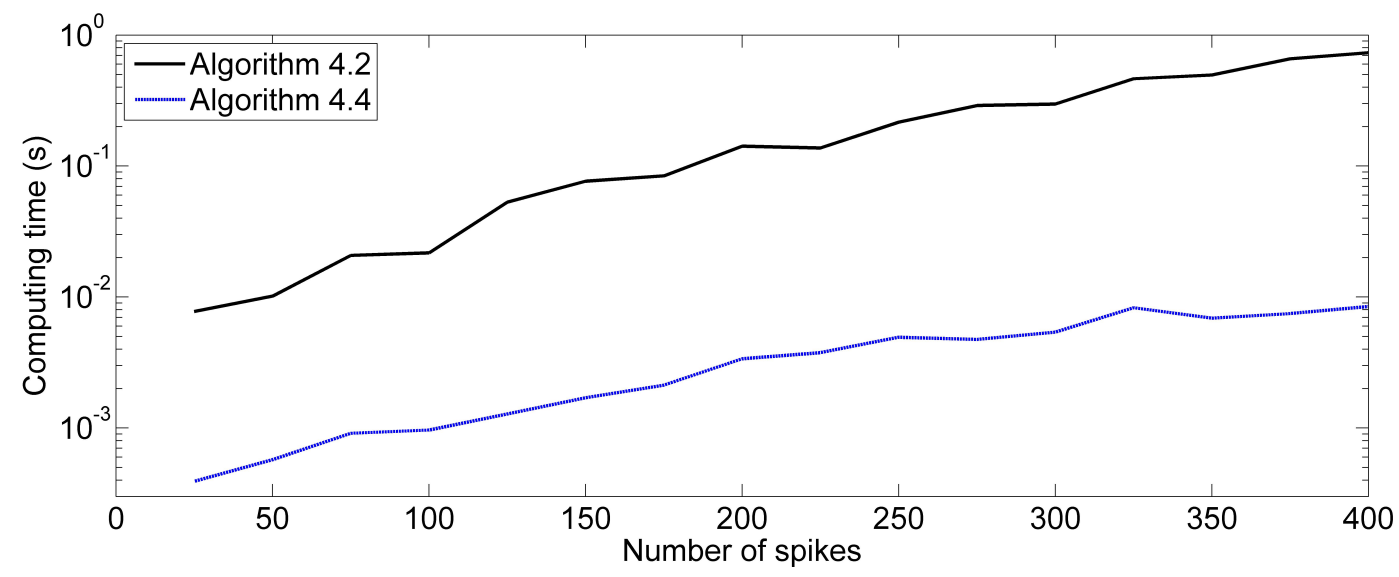

Figure 4.5: Comparison between Algorithm 4.2 and Algorithm 4.4. Probability density functions corresponding to: A) reconstruction time and B) reconstruction accuracy.

This chapter reformulated the problem of IF-TEM encoding as one of input independent uniform sampling. New results were introduced in order to show the advantage of the new formulation.

The direct method proposed in (Gontier and Vetterli, 2014) was generalised for reconstructing inputs $u \in V_{T}^{2}(\lambda)$ of the IF-TEM from the output sequence $\mathcal{I} \mathcal{T}_{u}$. A new noniterative direct method was then developed, which was proven to be more accurate than the iterative one. Two direct algorithms were developed to implement the two methods, respectively, for the particular case of the ideal IF neuron.

An iterative indirect method and a non-iterative indirect method were then introduced based on the new formulation of the IF-TEM encoding. Two indirect algorithms were developed to implement these new methods for the particular case of the ideal IF neuron.

The direct and indirect methods were compared through numerical simulations, for functions belonging to shift-invariant spaces generated by B-splines of degree 3 . The results show that all algorithms perform with a similar degree of accuracy. However, the indirect algorithms are up to 2 orders of magnitude faster than the corresponding direct algorithms.

The increase in computation time as a function of the number of spikes used in reconstruction is significantly higher for the direct methods. Moreover, as opposed to Algorithm 4.3, the rate of increase in the computation time of Algorithm 4.1 is dependent on the number of iterations used in reconstruction. 


\section{Chapter 5}

\section{A new approach to the identification of sensory processing circuits based on spiking neuron data}

System identification has been proven to be a powerful tool for developing quantitative models in sensory neurophysiology (Wu et al., 2006). Sensory processing systems have been identified using detailed biophysical models (Gu et al., 2009, Song et al., 2009, 2012) as well as phenomenological models (Friederich et al., 2009a, Geffen et al., 2009, Kim et al., 2011, Pillow and Simoncelli, 2006, Slee et al., 2005).

Identification methods have been developed for sensory processing circuits consisting of filters in cascade with spiking neurons, where the filter models the dendritic processing (Lazar and Slutskiy, 2010) or a receptive field (Lazar and Slutskiy, 2014b). These circuits have also been identified using recordings from the rat retina in order to develop prototypes for retinal prosthetic devices (Nirenberg and Pandarinath, 2012).

Lazar and Slutskiy (2010) have proposed a phenomenological model for characterising sensory systems, consisting of a linear filter in cascade with an ideal integrate-and-fire (IF) neuron. They have estimated the IF parameters of an input-output equivalent [Filter]-[ideal IF] circuit, and identified the filter with arbitrary precision by reformulating the identification problem as one of stimulus reconstruction. This result was extended to circuits with multiple inputs of one and multiple dimensions (Lazar and Slutskiy, 2013, 2012), and circuits with nonlinear filters that can be represented as a Volterra series (Lazar and Slutskiy, 2014c).

An identification method for [Fitler]-[Leaky IF] circuits has been proposed assuming that the Leaky IF neuron model is known a priori (Lazar and Slutskiy, 2010). Lazar and Slutskiy (2014b) have developed an algorithm for the full identification of [Fitler]-[HodgkinHuxley] circuits under the assumption that input-output measurements of the spiking neuron 
are available.

Although the identification methods above can accommodate a wide range of filters and spiking neurons, the assumptions proposed can sometimes be considered restrictive. Furthermore, the filter identification approach suffers from the well known practical limitation related to the problem of identifying Volterra kernels directly from data (Chen and Billings, 1989). An alternative to the methods above involves the reconstruction of the IF neuron input from the output spike train, and subsequently identifying the nonlinear filter. The NARMAX methodology, introduced by Leontaritis et al. (1981), is more general than the Volterra model, which was proven to be a useful tool for the identification of nonlinear biological systems (Korenberg and Hunter, 1996). This model has been successfully applied to characterize systems in neurophysiology (Friederich et al., 2010, 2009b) and has a wide range of applications, in fields as medicine (Linkens and Khelfa, 1992, Wei et al., 2009), geophysics (Coca et al., 2000, Zhu et al., 2008), environmental studies (Bartolini et al., 2008, Pisoni et al., 2009) and economy (Santos et al., 2007).

Two new identification methodologies are proposed for neural circuits comprising a linear or nonlinear filter in cascade with a spiking neuron.

A [Nonlinear Filter]-[Ideal IF] circuit is reformulated as a scaled nonlinear filter in series with a modified ideal IF neuron. The identification is subsequently carried out by employing the NARMAX nonlinear system identification methodology to infer the structure and parameters of a discrete-time representation for the scaled nonlinear filter. Numerical simulations are given to test the performance of the method.

An equivalent [Linear Filter]-[Leaky IF] circuit is identified, assuming that input-output measurements of the spiking neuron are not available and that all parameters are unknown. The leaky IF model is identified by solving an equation with a unique solution. An algorithm is provided that computes the solution with arbitrary precision. Subsequently, the structure and parameters of the filter are inferred using the NARMAX identification methodology. Numerical simulations are given to test the performance of the method.

This chapter is structured as follows. Section 5.1 contains a brief review of the identification methods for sensory processing circuits. An overview of the NARMAX methodology is given Section 5.2. A new identification methodology for [Nonlinear Filter]-[Ideal IF] circuits is proposed in Section 5.3. Section 5.4 introduces a new identification method for [Linear Filter]-[Leaky IF] circuits. Conclusions are in Section 5.5.

\subsection{Identification of spiking neural circuits}

This section reviews the identification results for [Filter]-[Spiking Neuron] circuits. Subsection 5.1.1 gives an overview of the methodology introduced in (Lazar and Slutskiy, 2010) for the identification of single-input single-output (SISO) [Linear Filter]-[Ideal IF] circuits. 
Generalizations of this method are reviewed in Subsection 5.1.2.

\subsubsection{Identification of [Linear Filter]-[Ideal IF] circuits}

Lazar and Slutskiy (2010) proposed a model consisting of a filter in cascade with an ideal IF neuron, depicted in Figure 5.1. The filter $h$ models the processing taking place in the neural dendritic tree. The ideal IF neuron consists of an adder and an ideal integrator that is reset to 0 every time the output of the adder reaches threshold value $\delta$.

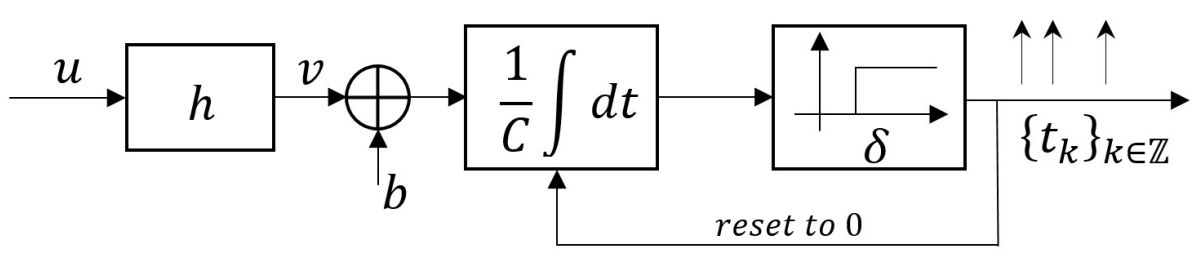

Figure 5.1: Linear filter in cascade with an IF neuron, modelling the dendritic tree and the spiking neuron, respectively.

The circuit is described by the following equation (Lazar and Pnevmatikakis, 2008a)

$$
\int_{t_{k}}^{t_{k+1}}(h * u)(\tau) d \tau=q_{k}, \forall k \in \mathbb{Z}
$$

where $q_{k}=\bar{\delta}-b\left(t_{k+1}-t_{k}\right), \bar{\delta}=C \delta, \forall k \in \mathbb{Z}$, and $\delta, C$ and $b$ denote the neuron threshold, integration constant and bias, respectively.

Input $u$ is assumed to be bandlimited $u \in P W_{\Omega}$. Moreover, it is assumed that filter $h$ satisfies $\operatorname{supp}(h) \subseteq\left[T_{1}, T_{2}\right], T_{1}, T_{2} \in \mathbb{R}$, and is bounded-input bounded-output (BIBO)stable, i.e.,

$$
\int_{-\infty}^{\infty}|h(\tau)| d \tau \leq \infty
$$

Under the assumptions presented above, Lazar and Slutskiy (2010) proposed the problem of identifying an input-output equivalent model of the circuit depicted in Figure 5.1, i.e., a model that triggers the same spike train $\left\{t_{k}\right\}_{k \in \mathbb{Z}}$ as the original model, when presented with the same input $u$, for any given bandwidth $\Omega>0$.

The equivalent model proposed by Lazar and Slutskiy (2010), depicted in Figure 5.2, is described by the following equation

$$
\int_{t_{k}}^{t_{k+1}}\left(h_{b} * u\right)(\tau) d \tau=\bar{\delta}_{b}-\left(t_{k+1}-t_{k}\right), \forall k \in \mathbb{Z}
$$

where $\bar{\delta}_{b} \triangleq \bar{\delta} / b$ and $h_{b} \triangleq h / b$. 


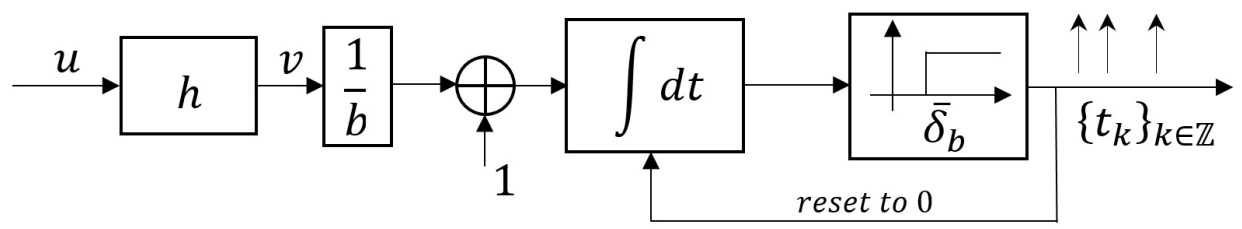

Figure 5.2: Input-output equivalent model for the [Filter]-[Ideal IF] circuit.

The identification methodology of the equivalent circuit proposed in (Lazar and Slutskiy, 2010) comprises two steps.

Firstly, the IF neuron parameter $\bar{\delta}_{b}$ is calculated as

$$
\bar{\delta}_{b}=t_{2}^{0}-t_{1}^{0}
$$

where $\left\{t_{k}^{0}\right\}_{k \in \mathbb{Z}}$ denotes the spike train triggered by the circuit when presented with input $u=0$.

Secondly, a sequence of inputs $\left\{u^{r}\right\}_{r=1, \ldots, N}, u^{r} \in P W_{\Omega}, r=1, \ldots, N$, is generated for the identification of filter $h_{b}$, such that $\left\{u^{r}\right\}_{r=1, \ldots, N}$ are linearly independent, i.e., the following two statements are equivalent (Lazar and Slutskiy, 2010)

$$
\exists\left\{\alpha_{r}, \beta_{r}\right\}_{r=1, \ldots, N} \text { such that } \sum_{r=1}^{N} \alpha_{r} \cdot u^{r}\left(t+\beta_{r}\right)=0, \forall t \in \mathbb{R} \Leftrightarrow \alpha_{r}=0, \forall r=1, \ldots, N \text {. }
$$

The circuit generates spike time sequences $\left\{t_{k}^{r}\right\}_{r=1, \ldots, N}$ when presented with inputs $\left\{u^{r}\right\}_{r=1, \ldots, N}$, respectively. Due to the convolution commutativity in (5.1), it follows that the problem of identifying filter $h_{b}$ from sequences $\left\{u^{r}, t_{k}^{r}\right\}_{r=1, \ldots, N}$ can be reformulated as the one of reconstructing the input of the single-input multiple-output (SIMO) circuit depicted in Figure 5.3 (Lazar and Slutskiy, 2010).

Function $u^{r}$ is bandlimited of bandwidth $\Omega$. Therefore, due to convolution associativity, the $t$-transform of the SIMO satisfies

$\int_{t_{k}^{r}}^{t_{k+1}^{r}}\left(h_{b} * u^{r}\right)(\tau) d \tau=\int_{t_{k}^{r}}^{t_{k+1}^{r}}\left(h_{b} *\left(g_{\Omega} * u^{r}\right)\right)(\tau) d \tau=\int_{t_{k}^{r}}^{t_{k+1}^{r}}\left(\left(h_{b} * g_{\Omega}\right) * u^{r}\right)(\tau) d \tau=\bar{q}_{k}^{r}$,

where $\bar{q}_{k}^{r}=\bar{\delta}_{b}-\left(t_{k+1}^{r}-t_{k}^{r}\right), \forall k \in \mathbb{Z}, r=1, \ldots, N$, and $g_{\Omega}=\frac{\sin (\Omega \cdot)}{\pi \cdot}$.

Function $h_{b} * g_{\Omega}$ is bandlimited to $\Omega \mathrm{rad} / \mathrm{s}$. It follows that it can be reconstructed perfectly provided that (Lazar and Slutskiy, 2010)

$$
\frac{N}{\bar{\delta}_{b}}>\frac{\pi}{\Omega}
$$




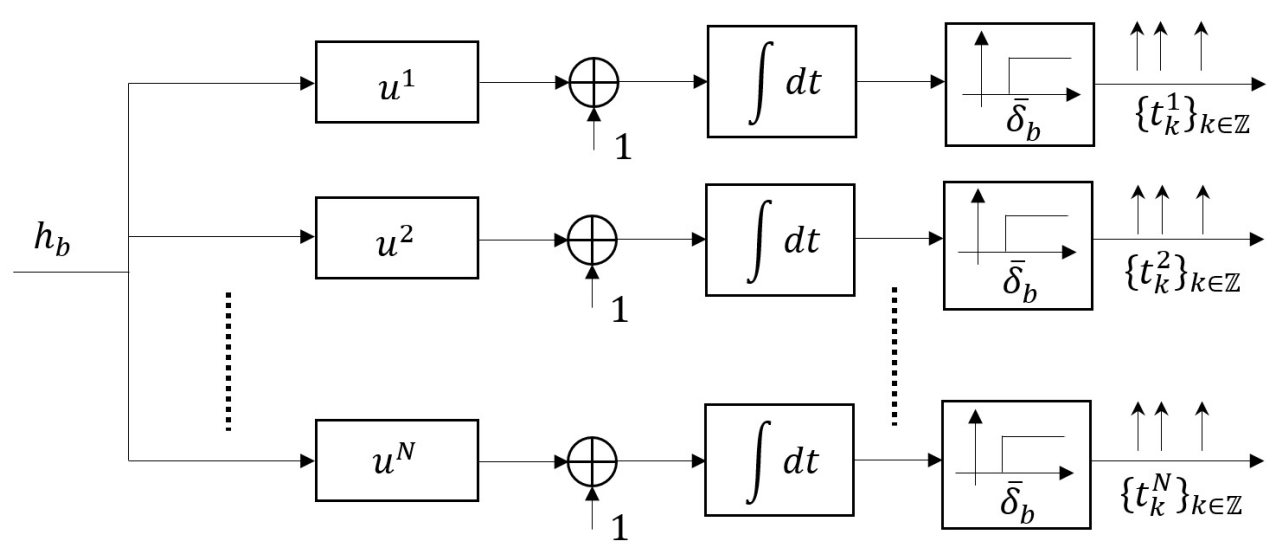

Figure 5.3: The estimation of $h_{b}$ reformulated as a reconstruction problem.

Therefore, for $\forall \Omega>0, \exists N \in \mathbb{N}^{*}$ such that the projection $\mathcal{P}_{\Omega} h_{b} \triangleq h_{b} * g_{\Omega}$ on the input space can be perfectly identified, where $\mathcal{P}_{\Omega}: L^{2}(\mathbb{R}) \rightarrow P W_{\Omega}$ denotes the orthogonal projection on $P W_{\Omega}$. The identification methodology of the filter, also known as the neuron identification machine (NIM), was presented in (Lazar and Slutskiy, 2010) and is summarised as follows.

Theorem 5.1. Let $\left\{u^{r}\right\}_{r=1, \ldots, N}$ denote a linearly independent sequence of functions such that $u^{r} \in P W_{\Omega}, r=1, \ldots, N$, and let $h \in H$, where $H$ is a Hilbert space, such that $\operatorname{supp}(h) \subseteq\left[T_{1}, T_{2}\right], T_{1}, T_{2} \in \mathbb{R}$. Let $\left\{t_{k}^{r}\right\}_{r=1, \ldots, N}$ denote the spike time sequences generated by the [Filter]-[Ideal IF] circuit when presented with inputs $\left\{u^{r}\right\}_{r=1, \ldots, N}$, respectively. Provided that $\frac{N}{\bar{\delta}_{b}}>\frac{\pi}{\Omega}, \mathcal{P}_{\Omega} h_{b}$ can be identified perfectly as

$$
\mathcal{P}_{\Omega} h_{b}=g c,
$$

where $c=\left[\boldsymbol{c}^{1}, \boldsymbol{c}^{2}, \ldots, \boldsymbol{c}^{N}\right]^{T}, \boldsymbol{c}^{j} \in \mathbb{R}^{\mathbb{Z} \times 1},\left[\boldsymbol{c}^{j}\right]_{k}=c_{k^{j}}^{j}, \forall j=1, \ldots, N, k \in \mathbb{Z}$ and $\boldsymbol{g}=$ $\left[\boldsymbol{g}^{\mathbf{1}}, \boldsymbol{g}^{\mathbf{2}}, \ldots, \boldsymbol{g}^{N}\right], \boldsymbol{g}^{j} \in \mathbb{R}^{1 \times \mathbb{Z}},\left[\boldsymbol{g}^{j}\right]_{k}=g_{\Omega}\left(\cdot-t_{k}^{j}\right), \forall j=1, \ldots, N, k \in \mathbb{Z}$.

Coefficients $c$ are calculated as

$$
c=G^{+} \boldsymbol{q}
$$

where $G^{+}$denotes the Moore-Penrose pseudoinverse of matrix $G$ and

$$
G=\left[\begin{array}{cccc}
G^{11} & G^{12} & \ldots & G^{1 N} \\
G^{21} & G^{22} & \ldots & G^{2 N} \\
\ldots & \ldots & \ldots & \ldots \\
G^{N 1} & G^{N 2} & \ldots & G^{N N}
\end{array}\right], q=\left[\begin{array}{c}
q^{1} \\
q^{2} \\
\ldots \\
q^{N}
\end{array}\right]
$$

where $G^{r j} \in \mathbb{R}^{\mathbb{Z} \times \mathbb{Z}}, \boldsymbol{q}^{j} \in \mathbb{R}^{\mathbb{Z} \times 1},\left[\boldsymbol{G}^{r j}\right]_{l k}=\int_{t_{l}^{r}}^{t_{l+1}^{r}} u^{r}\left(\tau-t_{k}^{j}\right) d \tau,\left[\boldsymbol{q}^{j}\right]_{k}=\bar{q}_{k}^{j}, \forall r, j=$ 
$1, \ldots, N, \forall l, k \in \mathbb{Z}$

Lazar and Slutskiy (2010) have shown that $\mathcal{P}_{\Omega} h$ can be identified perfectly for circuits with different spiking neuron models. For [Filter]-[Leaky IF] circuits, Theorem 5.1 can be applied with

$$
\left[\boldsymbol{G}^{r j}\right]_{l k}=\int_{t_{l}^{r}}^{t_{l+1}^{r}} u^{r}\left(\tau-t_{k}^{j}\right) e^{\frac{\tau-t_{l+1}^{r}}{R C}} d \tau,\left[\boldsymbol{q}^{j}\right]_{l}=C \delta-b R C\left[1-e^{\frac{t_{l}^{j}-t_{l+1}^{j}}{R C}}\right] .
$$

However, this result assumes that the leaky IF neuron parameters are known a priori. In contrast to [Filter]-[Ideal IF] circuits, here it is not possible to design an input-output equivalent model whose spiking neuron parameters can be identified using a single measurement.

\subsubsection{Identification methods for different circuit structures}

Lazar and Slutskiy (2012) have applied the methodology introduced in (Lazar and Slutskiy, 2010) for the identification of a filter, representing the communication channel, in a [Filter]-[Ideal IF] circuit. The algorithm used, also known as channel identification machine (CIM) was then extended to the estimation of several filters in a multiple-input single-output (MISO) circuit (Lazar and Slutskiy, 2012).

To account for the multisensory integration taking place in the brain, Lazar and Slutskiy (2013) proposed the problem of identifying the filters in a MISO [Filter]-[Ideal IF] circuit with multidimensional inputs. The proposed circuit is depicted in Figure 5.4 .

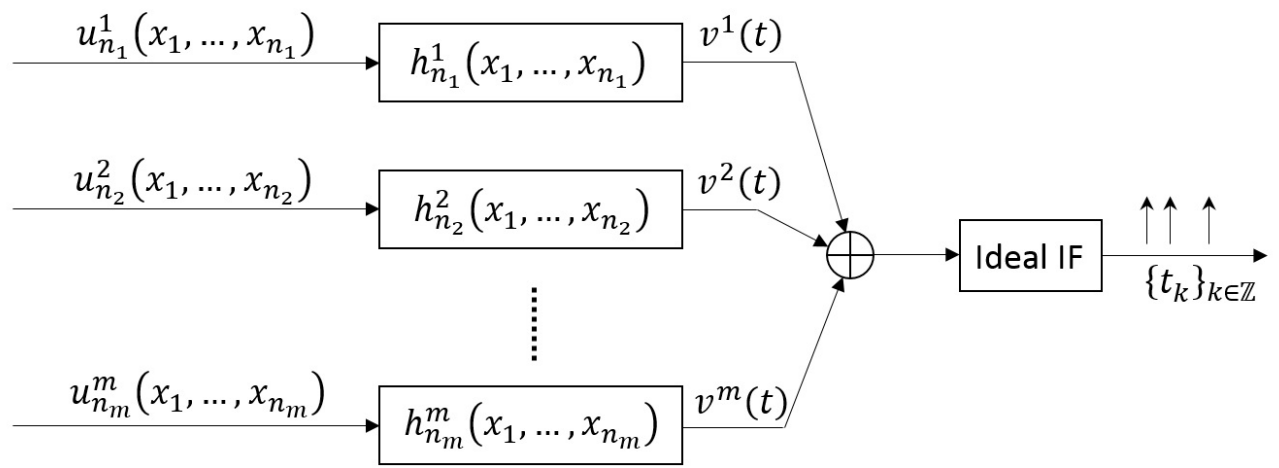

Figure 5.4: MISO [Filter]-[Ideal IF] circuit with multisensory integration.

Here, the input functions satisfy $u_{n_{r}}^{r} \in \mathcal{H}_{\Omega}\left(\mathbb{D}_{n_{r}}\right)$, where $\mathbb{D}_{n_{r}}=\prod_{p=1}^{n_{r}}\left[0, T_{p}\right]$, and $\mathcal{H}_{\Omega}\left(\mathbb{D}_{n_{r}}\right)=\left\{u: \mathbb{D}_{n_{r}} \rightarrow \mathbb{C}: u\left(x_{1}, \ldots, x_{n_{r}}\right)=\sum_{l_{1}=-L_{1}}^{L_{1}} \ldots \sum_{l_{n_{r}}=-L_{n_{r}}}^{L_{n_{r}}} u_{l_{1} \ldots l_{n_{r}}} e^{\sum_{p=1}^{n_{r}} j l_{p} \Omega_{p} x_{p} / L_{p}}\right\}$. 
The space $\mathcal{H}_{\Omega}\left(\mathbb{D}_{n_{r}}\right)$ represents the space of trigonometric polynomials of $n_{r}$ dimensions, and $T_{p}$ and $\Omega_{p}$ are the period and bandwidth in dimension $x_{p}$, respectively. The filters are assumed to satisfy $h_{n_{r}}^{r} \in H_{n_{r}}$, where

$$
H_{n_{r}} \triangleq\left\{h \in L^{1}\left(\mathbb{R}^{n_{r}}\right): \operatorname{supp}(h) \subseteq \mathbb{D}_{n_{r}}\right\}, \forall r=1, \ldots, m
$$

The $t$-transform of this circuit is (Lazar and Slutskiy, 2013)

$$
\int_{t_{k}}^{t_{k+1}} \sum_{r=1}^{m} v^{r}(\tau) d \tau=C \delta-b\left(t_{k+1}-t_{k}\right), \forall k \in \mathbb{Z},
$$

where functions $\left\{v^{r}\right\}_{r=1, \ldots, m}$ satisfy

$$
v^{r}(t)=\int_{\mathbb{D}_{n_{r}}} h_{n_{r}}^{r}\left(x_{1}, \ldots, x_{n_{r}-1}, \tau\right) u_{n_{r}}^{r}\left(x_{1}, \ldots, x_{n_{r}-1}, t-\tau\right) d x_{1} \ldots d_{x_{n_{r}-1}} d \tau
$$

The identification problem is reformulated as one of reconstruction of the multidimensional filters $\left\{h_{n_{r}}^{r}\right\}_{r=1, \ldots, m}$. Lazar and Slutskiy (2013) have proven that only a projection of the filters on the input space $\mathcal{H}_{\Omega}\left(\mathbb{D}_{n_{r}}\right)$ can be perfectly identified. The methodology proposed, called the multisensory CIM (mCIM) (Lazar and Slutskiy, 2013), is a generalization of the NIM (Lazar and Slutskiy, 2010) and of the CIM (Lazar and Slutskiy, 2012).

Lazar and Slutskiy (2014a) have applied the mCIM method to particular types of inputs, namely spatial $u(x, y)$, spectrotemporal $u(v, t)$ and spatiotemporal functions $u(x, y, t)$. Lazar et al. (2015) have introduced a particular type of CIM called colour video CIM, where the circuit input is a colour visual stimulus modelled as a vector-valued function $\boldsymbol{u}(x, y, t)=\left[u_{1}(x, y, t), u_{2}(x, y, t), u_{3}(x, y, t)\right]$.

Lazar and Slutskiy (2014b) considered the problem of identifying a [Linear Filter][Hodgkin-Huxley Neuron] circuit. The Hodgkin-Huxley model is described by the following set of equations (Hodgkin and Huxley, 1952)

$$
\begin{aligned}
C \frac{d V}{d t} & =-g_{N a} m^{3} h\left(V-E_{N a}\right)-g_{K} n^{4}\left(V-E_{K}\right)-g_{L}\left(V-E_{L}\right)+I \\
\frac{d m}{d t} & =\alpha_{m}(V)(1-m)-\beta_{m}(V) m \\
\frac{d h}{d t} & =\alpha_{H}(V)(1-h)-\beta_{h}(V) h \\
\frac{d n}{d t} & =\alpha_{n}(V)(1-n)-\beta_{n}(V) n,
\end{aligned}
$$

where $V$ represents the membrane voltage, $m, h$, and $n$ are the gating variables and $I$ is the injected current. Lazar and Slutskiy (2014b) have proven that this model is input-output 
equivalent to a project IF neuron, described by the following $t$ - transform

$$
\int_{t_{k}}^{t_{k+1}} \varphi^{1}\left(\tau-t_{k}\right) v(\tau) d \tau=\delta-\left(t_{k+1}-t_{k}\right), \forall k \in \mathbb{Z},
$$

where $v$ is the filter output and $\varphi^{1}$ denotes the phase response curve (PRC) of the neuron on a stable orbit. An identification methodology was proposed, consisting of estimating the PRC curve and subsequently identifying the filter using the CIM algorithm.

Lazar and Slutskiy (2014c) have proposed a methodology for the identification of [Nonlinear Filter]-[Ideal IF] circuits. The filters, called dendritic stimulus processors (DSPs), are approximated with their truncated Volterra expansion of order $P$ (Volterra, 2005)

$$
\begin{aligned}
v(t) & =\int_{\mathbb{D}} h^{1}\left(t-\tau_{1}\right) u\left(\tau_{1}\right) d \tau_{1}+\int_{\mathbb{D}^{2}} h^{2}\left(t-\tau_{1}, t-\tau_{2}\right) u\left(\tau_{1}\right) u\left(\tau_{2}\right) d \tau_{1} d \tau_{2}+\ldots \\
& +\int_{\mathbb{D}^{P}} h^{P}\left(t-\tau_{1}, \ldots, t-\tau_{P}\right) u\left(\tau_{1}\right) \cdots u\left(\tau_{P}\right) d \tau_{1} \ldots d \tau_{P} .
\end{aligned}
$$

Let $u^{p}\left(t_{1}, \ldots, t_{p}\right) \triangleq u\left(t_{1}\right) \cdots u\left(t_{p}\right), p=1, \ldots, P$. The resulting [Filter]-[Ideal IF] circuit is depicted in Figure 5.5.

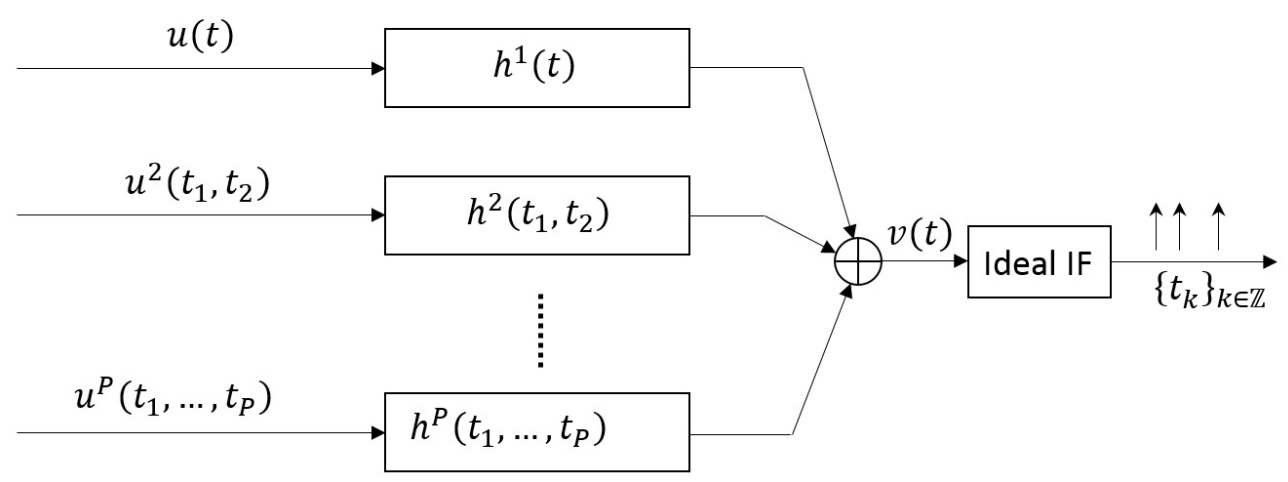

Figure 5.5: Volterra expansion of the filter in a [Nonlinear Filter]-[Ideal IF] circuit.

Therefore, the model represents a MISO [Linear Filter]-[Ideal IF] circuit with multidimensional inputs. The identification method, called Volterra CIM, identifies perfectly the multidimensional projections of the Volterra kernels $\left\{h^{p}\right\}_{p=1, \ldots, P}$ onto the corresponding input spaces (Lazar and Slutskiy, 2014c). 


\subsection{The NARMAX identification methodology}

A well established methodology for the identification of linear as well as nonlinear systems involves the use of the NARMAX (Nonlinear AutoRegressive Moving Average with eXogenous inputs) representation (Billings and Leontaritis, 1982, Leontaritis and Billings, 1985a,b, Leontaritis et al., 1981). This model is a generalisation of the ARMAX (AutoRegressive Moving Average with eXogenous inputs) model, designed for linear systems (Akaike, 1969, 1970, Fung et al., 2003).

The NARMAX methodology provides a suitable representation for a wide range of nonlinear systems, including as particular cases functional series models such as the Volterra model (Billings and Coca, 2002, Chen and Billings, 1989).

The NARMAX representation, as compared with Volterra, requires a relatively low number of parameters, and thus short data lengths, for capturing the dynamics of highly nonlinear systems (Chen and Billings, 1989, Diaz and Desrochers, 1988). Moreover, spectral analysis can be performed for nonlinear system through their NARMAX representation by computing the generalised frequency response functions (GFRFs) (Billings and Tsang, 1989a,b, Billings et al., 1990, Chua and Ng, 1979a,b, Jones, 2007, Lee and Chang, 2009, Zhang and Billings, 1993).

\subsubsection{An overview of the NARMAX model}

The NARMAX input/output representation is defined by (Pearson, 1995, 1999, Thomson et al., 1996)

$$
\begin{aligned}
y(k)= & F\left[y(k-1), \ldots, y\left(k-n_{y}\right), u(k-1), \ldots, u\left(k-n_{u}\right),\right. \\
& \left.\quad(k-1), e(k-2), \ldots, e\left(k-n_{e}\right)\right]+e(k), k=1, \ldots, N,
\end{aligned}
$$

where $u$ and $y$ are discrete sequences representing the input and output, respectively, $e$ represents the combined effects of measurement noise, modelling errors and unmeasured disturbances, $n_{u}, n_{y}$, and $n_{e}$ denote the maximum lags for $u, y$ and $e$, respectively, and $F$ is a nonlinear function. A particular form is the NARX (Nonlinear AutoRegressive with eXogenous inputs) model

$$
y(k)=F\left[y(k-1), \ldots, y\left(k-n_{y}\right), u(k-1), \ldots, u\left(k-n_{u}\right)\right]+e(k), k=1, \ldots, N, .
$$

The nonlinear form of function $F$ is generally unknown, or difficult to determine. Chen and Billings (1989) have proven that if $F$ is continuous, it can be approximated with arbitrary precision by a polynomial function 


$$
F[x(k)]=\sum_{j=1}^{M} \theta_{j} p_{j}(x(k)), k=1, \ldots, N,
$$

where $x(k)=\left[y(k-1), \ldots, y\left(k-n_{y}\right), u(k-1), \ldots, u\left(k-n_{u}\right)\right], p_{j}$ is a monomial of degree $d_{j}<l$, and $\theta_{j} \in \mathbb{R}, \forall j=1, \ldots, M$.

The linear-in-the-parameters representation of the NARX model is given by equation (5.2) where $F$ satisfies (5.3), and has the following matrix representation (Billings, 2013)

$$
Y=P \theta+e
$$

where

$$
\begin{aligned}
\boldsymbol{Y} & \triangleq[y(1), \ldots, y(N)] \\
\boldsymbol{\theta} & \triangleq\left[\theta_{1}, \ldots, \theta_{N}\right] \\
\boldsymbol{e} & \triangleq[e(1), \ldots, e(N)] \\
\boldsymbol{P} & \triangleq\left[\boldsymbol{p}_{1}, \ldots, \boldsymbol{p}_{M}\right] \\
\boldsymbol{p}_{\boldsymbol{i}} & \triangleq\left[p_{i}(x(1)), \ldots, p_{i}(x(N))\right]^{T}, i=1, \ldots, M .
\end{aligned}
$$

The vectors $\left\{\boldsymbol{p}_{\boldsymbol{i}}\right\}_{i=1, \ldots, M}$ are also known as the regressors or terms of NARX representation (5.4). The coefficients are estimated as

$$
\hat{\boldsymbol{\theta}}=\min _{\boldsymbol{\theta}}\|\boldsymbol{Y}-\boldsymbol{P} \boldsymbol{\theta}\|_{\mathbb{R}^{N}}^{2}
$$

where $\|\cdot\|_{\mathbb{R}^{N}}$ denotes the vector norm in $\mathbb{R}^{N}$.

The total number of terms $M$ satisfies

$$
M=\frac{(n+l) !}{n ! \cdot l !}
$$

where $n=n_{y}+n_{u}$ and $n$ ! denotes the factorial function, i.e., $n !=1 \cdot 2 \cdots n$. The number of terms $M$ becomes very large for large values of $n$.

\subsubsection{The orthogonal least squares estimator}

The Orthogonal Least Squares (OLS) estimator was introduced to overcome the problem of large number of terms, by eliminating the ones that don't have a significant contribution to output $Y$ (Billings and Chen, 1989, Billings et al., 1988, Chen et al., 1989, Korenberg et al., 1988).

Under the assumption that vectors $\left\{p_{l}\right\}_{l=1, \ldots, N}$ are linearly independent, this method 
introduces an auxiliary linear-in-the-parameters representation

$$
y(k)=\sum_{s=1}^{M} g_{s} w_{s}(k)+e(k)
$$

where $w_{s} \triangleq\left[w_{s}(1), \ldots, w_{s}(N)\right]^{T}, s=1, \ldots, M$ satisfy (Billings, 2013)

$$
\begin{aligned}
& w_{1}=p_{1} \\
& w_{s}=p_{s}-\sum_{r=1}^{s-1} a_{r, s} w_{r}, s=2, \ldots, M,
\end{aligned}
$$

where

$$
a_{r, s} \triangleq \frac{\left\langle p_{s}, w_{r}\right\rangle_{\mathbb{R}^{N}}}{\left\|w_{r}\right\|_{\mathbb{R}^{N}}^{2}}, r=1, \ldots, M-1, s=2, \ldots, M, s>r
$$

Coefficients $\left\{g_{s}\right\}_{s=1, \ldots, M}$ are calculated as

$$
g_{s}=\frac{\left\langle\boldsymbol{Y}, \boldsymbol{w}_{s}\right\rangle_{\mathbb{R}^{N}}}{\left\|\boldsymbol{w}_{\boldsymbol{s}}\right\|_{\mathbb{R}^{N}}^{2}}, s=1, \ldots, M
$$

Equations (5.5) perform the Gram-Schmidt orthogonalization of vectors $\left\{\boldsymbol{p}_{\boldsymbol{s}}\right\}_{s=1, \ldots, M}$, which, in matrix form, is equivalent in this case to the $\mathrm{QR}$ factorization of matrix $\boldsymbol{P}$ (Trefethen and Bau III, 1997)

$$
\boldsymbol{P}=W \cdot A,
$$

where $W$ denotes the orthogonal matrix $W \triangleq\left[w_{1}, \ldots, w_{M}\right]$ and $A$ is an upper triangular matrix satisfying

$$
A=\left[\begin{array}{cccc}
1 & a_{1,2} & \ldots & a_{1, M} \\
0 & 1 & \ldots & a_{2, M} \\
\ldots & \ldots & \ldots & \ldots \\
0 & 0 & \ldots & 1
\end{array}\right]
$$

Therefore the relationship between the original and the auxiliary NARX representations is given by (Chen, 2006)

$$
Y=W(A \theta)+e=W g+e,
$$

where $g \triangleq\left[g_{1}, \ldots, g_{M}\right]^{T}$, and thus

$$
\boldsymbol{\theta}=A^{-1} g
$$


The output energy $\frac{1}{N}\|\boldsymbol{Y}\|_{\mathbb{R}^{N}}^{2}$ of the NARX representation satisfies (Billings, 2013)

$$
\frac{1}{N}\|\boldsymbol{Y}\|_{\mathbb{R}^{N}}^{2}=\frac{1}{N} \sum_{s=1}^{M}\left\|g_{s} \boldsymbol{w}_{s}\right\|_{\mathbb{R}^{N}}^{2}+\frac{1}{N}\|\boldsymbol{e}\|_{\mathbb{R}^{N}}^{2}
$$

In order to identify the significant regressors, i.e., the regressors that have a significant contribution to the total output energy, the error-to-signal ration (ESR) criterion is defined as (Billings, 2013)

$$
E S R \triangleq 1-\sum_{s=1}^{M} E R R_{s}
$$

where $E R R_{s}$ denotes the error reduction ratio (ERR) of regressor $w_{s}$

$$
E R R_{s} \triangleq \frac{\left\|g_{s} \boldsymbol{w}_{s}\right\|_{\mathbb{R}^{N}}^{2}}{\|\boldsymbol{Y}\|_{\mathbb{R}^{N}}^{2}}, s=1, \ldots, M
$$

The significant regressors can thus be identified as the ones for which $E R R_{S}$ is above a selected threshold value and the overall ESR is negligible.

\subsubsection{The orthogonal forward regression algorithm}

The ordering of regressors according to the $E R R$ criterion is sensitive to changing their positions in the $\left\{\boldsymbol{p}_{s}\right\}_{s=1, \ldots, M}$ sequence. The Forward Regression OLS algorithm, also known as Orthogonal Forward Regression (OFR) has been proposed to overcome this problem (Billings et al., 1988, 1989). The OFR algorithm is a modified version of the OLS method, in which every step additionally includes searching for the most significant regressor from the unselected model terms (Guo and Billings, 2007, Hong et al., 2008a,b).

Let $D$ denote the full set of regressors $D=\left\{\boldsymbol{p}_{s}\right\}_{s=1, \ldots, M}$, also known as dictionary. The algorithm computes a subset of significant regressors $D_{m} \subseteq D, D_{m}=\left\{\boldsymbol{p}_{\ell_{s}}\right\}_{s=1, \ldots, m}, m \leq$ $M$. The index of the first regressor is the solution to the minimization problem

$$
\ell_{1}=\underset{j=1, \ldots, M}{\operatorname{argmax}} \frac{\left\|g_{j}^{(1)} \boldsymbol{p}_{j}\right\|_{\mathbb{R}^{N}}^{2}}{\|\boldsymbol{Y}\|_{\mathbb{R}^{N}}^{2}},
$$

where

$$
g_{j}^{(1)}=\frac{\left\langle\boldsymbol{Y}, \boldsymbol{p}_{j}\right\rangle_{\mathbb{R}^{N}}}{\left\|\boldsymbol{p}_{j}\right\|_{\mathbb{R}^{N}}^{2}}, j=1, \ldots, M .
$$

Let $L^{(s)} \triangleq\left\{\ell_{1}, \ldots, \ell_{s}\right\}, s=1, \ldots, M$, and let $\boldsymbol{q}_{1}=\boldsymbol{p}_{\ell_{1}}$. For $s=2, \ldots, M$, compute

$$
\boldsymbol{q}_{j}^{(s)}=\boldsymbol{p}_{\boldsymbol{s}}-\sum_{r=1}^{s-1} \frac{\left\langle\boldsymbol{p}_{j}, \boldsymbol{q}_{r}\right\rangle_{\mathbb{R}^{N}}}{\left\|\boldsymbol{q}_{\boldsymbol{r}}\right\|_{\mathbb{R}^{N}}^{2}} \boldsymbol{q}_{r}, j \in\{1, \ldots, M\} \backslash L^{(s-1)},
$$




$$
\begin{gathered}
\ell_{s}=\underset{\substack{j=1, \ldots, M \\
j \notin L^{(s)}}}{\operatorname{argmax}} \frac{\left\|g_{j}^{(s)} \boldsymbol{p}_{j}\right\|_{\mathbb{R}^{N}}^{2}}{\|\boldsymbol{Y}\|_{\mathbb{R}^{N}}^{2}}, \\
E R R_{s}=\frac{\left\|g_{\ell_{s}}^{(s)} \boldsymbol{p}_{\ell_{s}}\right\|_{\mathbb{R}^{N}}^{2}}{\|\boldsymbol{Y}\|_{\mathbb{R}^{N}}^{2}}, \\
\boldsymbol{q}_{s} \triangleq \boldsymbol{q}_{\ell_{s}}^{(s)}, g_{s} \triangleq g_{\ell_{s}}^{(s)},
\end{gathered}
$$

where

$$
g_{j}^{(s)}=\frac{\left\langle\boldsymbol{Y}, \boldsymbol{q}_{j}^{(s)}\right\rangle_{\mathbb{R}^{N}}}{\left\|\boldsymbol{q}_{j}^{(s)}\right\|_{\mathbb{R}^{N}}^{2}}, j \in\{1, \ldots, M\} \backslash L^{(s-1)} .
$$

The algorithm terminates at $s=m$, which satisfies

$$
E S R_{m}=1-\sum_{s=1}^{m} E R R_{s} \leq \varepsilon
$$

where $\varepsilon$ is the selected threshold value.

This algorithm computes a set of regressors $\left\{p_{\ell_{i}}\right\}_{s=1, \ldots, m}$, ordered from the most to the least significant, and its corresponding set of orthogonalised vectors $\left\{\boldsymbol{q}_{s}\right\}_{s=1, \ldots, m}$. The final model is

$$
y(k)=\sum_{s=1}^{m} \theta_{\ell_{s}} p_{\ell_{s}}(k)+e(k), k=1, \ldots, N .
$$

The parameters $\boldsymbol{\theta}=\left[\theta_{\ell_{1}}, \theta_{\ell_{2}}, \ldots, \theta_{\ell_{m}}\right]$ satisfy

$$
\boldsymbol{\theta}=A^{-1} \boldsymbol{g}
$$

where $g \triangleq\left[g_{1}, \ldots, g_{M}\right]^{T}$ and

$$
A=\left[\begin{array}{cccc}
1 & a_{1,2} & \ldots & a_{1, m} \\
0 & 1 & \ldots & a_{2, m} \\
\ldots & \ldots & \ldots & \ldots \\
0 & 0 & \ldots & 1
\end{array}\right]
$$

where

$$
a_{r, s} \triangleq \frac{\left\langle\boldsymbol{p}_{\ell_{s}}, \boldsymbol{q}_{\boldsymbol{r}}\right\rangle_{\mathbb{R}^{N}}}{\left\|\boldsymbol{q}_{\boldsymbol{r}}\right\|_{\mathbb{R}^{N}}^{2}}, r=1, \ldots, m-1, s=2, \ldots, m, s>r .
$$

The data used for identification is typically split in two parts, i.e., the estimation data set and test data set (Billings and Fadzil, 1984). The former set is used for the estimation of the model parameters, and then the model is tested using the latter data set, generated using a distinct input signal (Billings, 2013). 
The One-Step-Ahead (OSA) prediction $\left\{\hat{y}_{O S A}(k)\right\}_{k=\left(n_{y}+1\right) \ldots, N}$ of the estimated NARMAX model with $n_{u}$ and $n_{y}$ input and output lags, respectively, is calculated as

$$
\hat{y}_{O S A}(k)=\sum_{j=1}^{M} \theta_{j} p_{j}\left(\left[y(k-1), \ldots, y\left(k-n_{y}\right), u(k-1), \ldots, u\left(k-n_{u}\right)\right]\right),
$$

for $k=\left(n_{y}+1\right), \ldots, N$, where $\{u(k)\}_{k=1, \ldots, N}$ and $\{y(k)\}_{k=1, \ldots, N}$ are the test input and output sequences, respectively.

Alternatively, the Model Predicted Output (MPO) $\left\{\hat{y}_{M P O}(k)\right\}_{k=\left(n_{y}+1\right) \ldots, N}$ of the same NARMAX model satisfies

$$
\hat{y}_{M P O}(k)=\sum_{j=1}^{M} \theta_{j} p_{j}\left(\left[\hat{y}_{M P O}(k-1), \ldots, \hat{y}_{M P O}\left(k-n_{y}\right), u(k-1), \ldots, u\left(k-n_{u}\right)\right]\right),
$$

for $k=\left(n_{y}+1\right), \ldots, N$.

The OSA prediction has the disadvantage of suppressing the build-up errors in the predicted output, which is why even a poor model can have a good OSA prediction. In contrast, the errors accumulate in the MPO, and thus it is more useful for determining when a model is inadequate (Wei et al., 2009).

To quantify how good a prediction is, the following errors are defined

$$
M S E=\frac{\sum_{k=1}^{N}(\hat{y}(k)-y(k))^{2}}{N}, R M S E=\sqrt{\frac{\sum_{k=1}^{N}(\hat{y}(k)-y(k))^{2}}{N}},
$$

where MSE and RMSE denote the mean squared error and the root mean squared error, respectively. To eliminate the dependency on the signal energy, the following error is defined

$$
N M S E=\frac{\sum_{k=1}^{N}(\hat{y}(k)-y(k))^{2}}{\sum_{k=1}^{N}(\bar{y}-y(k))^{2}},
$$

where NMSE denotes the normalised mean squared error, and $\bar{y} \triangleq \frac{\sum_{k=1}^{N} y(k)}{N}$ denotes the mean value of output $y$.

\subsubsection{The generalised frequency response functions}

The theory of generalised frequency response functions (GFRFs) is based on the theory of Volterra series for nonlinear systems. Boyd and Chua (1985) have proven that a continuous operator can be expanded into Volterra series if it has fading memory, i.e., the operator calculated in two functions that are distant in the remote past, but close in the recent past, yields two functions which are close in the present.

The Volterra series expansion for continuous-time nonlinear systems is (Volterra, 2005) 


$$
y(t)=\sum_{n=1}^{M} y_{n}(t)
$$

where $M$ is the maximum order of nonlinearity, and

$$
y_{n}(t)=\int_{-\infty}^{\infty} \ldots \int_{-\infty}^{\infty} h_{n}\left(\tau_{1}, \ldots, \tau_{n}\right) \prod_{s=1}^{n} u\left(t-\tau_{s}\right) d \tau_{s}
$$

The series (5.7) converges if functions $\left\{h_{n}\right\}_{n=1 \ldots, M}$, also known as the Volterra kernels, satisfy the following condition (Boyd and Chua, 1985)

$$
\int_{-\infty}^{\infty} \ldots \int_{-\infty}^{\infty}\left|h_{n}\left(\tau_{1}, \ldots, \tau_{n}\right)\right| d \tau_{1} \ldots d \tau_{n}<\infty, n=1, \ldots, M
$$

The following symmetric kernel is defined (Billings, 2013)

$$
h_{n}^{\text {sym }}\left(\tau_{1}, \ldots, \tau_{n}\right)=\frac{1}{n !} \sum_{\pi} h_{n}\left(\tau_{\pi(1)}, \ldots, \tau_{\pi(n)}\right),
$$

where $\pi$ denotes an arbitrary permutation of set $\{1,2 \ldots, n\}$. Without reducing the generality, this kernel can replace functions $\left\{h_{n}\right\}_{n=1, \ldots, M}$ in (5.7), which, therefore, are assumed from now on to be symmetric functions.

The GFRF of order $n$, denoted $H_{n}, n=1, \ldots, M$, is defined as (George, 1959)

$$
H_{n}\left(i \omega_{1}, \ldots, i \omega_{n}\right)=\int_{-\infty}^{\infty} \ldots \int_{-\infty}^{\infty} h_{n}\left(\tau_{1}, \ldots, \tau_{n}\right) e^{-i\left(\omega_{1} \tau_{1}+\cdots+\omega_{n} \tau_{n}\right)} d \tau_{1} \ldots d \tau_{n}
$$

for $n=1, \ldots, M$, where $i$ denotes the unit of the imaginary axis. Without reducing the generality, the continuous GFRFs are replaced by the following functions

$$
H_{n}^{s y m}\left(i \omega_{1}, \ldots, i \omega_{n}\right)=\frac{1}{n !} \sum_{\pi} H_{n}\left(i \omega_{\pi(1)}, \ldots, i \omega_{\pi(n)}\right)
$$

The output function $y$ satisfies (5.6), (5.7)

$$
y(t)=\sum_{n=1}^{M} \frac{1}{(2 \pi)^{n}} \int_{-\infty}^{\infty} \ldots \int_{-\infty}^{\infty} H_{n}\left(i \omega_{1}, \ldots, i \omega_{n}\right) \prod_{s=1}^{n} U\left(i \omega_{s}\right) e^{i\left(\omega_{1}+\cdots+\omega_{n}\right) t} d \omega_{s}
$$

The spectrum of $y(t)$ can be represented as a function of the GFRFs as (Lang and Billings, 1996)

$$
Y(i \omega)=\sum_{s=1}^{n} Y_{n}(i \omega)
$$


where $\omega=\omega_{1}+\cdots+\omega_{n}$ and

$$
Y_{n}(i \omega)=\frac{1}{\sqrt{n} \cdot \pi^{n-1}} \int_{\omega_{1}+\cdots+\omega_{n}=\omega} H_{n}\left(i \omega_{1}, \ldots, i \omega_{n}\right) \prod_{s=1}^{n} U\left(i \omega_{s}\right) e^{i\left(\omega_{1}+\cdots+\omega_{n}\right) t} d \sigma,
$$

where $d \sigma$ denotes the infinitesimal area on hyperplane $\omega=\omega_{1}+\cdots+\omega_{n}$.

The Volterra series expansion for discrete-time nonlinear systems is (Billings, 2013)

$$
y(k)=\sum_{n=1}^{M} y_{n}(k)
$$

where $M$ is the maximum order of nonlinearity, and

$$
y_{n}(k)=\sum_{s_{1}=0}^{\infty} \cdots \sum_{s_{n}=0}^{\infty} h_{n}\left(s_{1}, \ldots, s_{n}\right) u\left(k-s_{1}\right) \cdots u\left(k-s_{n}\right) .
$$

Functions $\left\{h_{n}\right\}_{n=1, \ldots, M}$ are required to satisfy following condition, which ensures the convergence of series (5.9)

$$
\sum_{s_{1}=0}^{\infty} \cdots \sum_{s_{n}=0}^{\infty}\left|h_{n}\left(s_{1}, \ldots, s_{n}\right)\right|<\infty, n=1, \ldots, M .
$$

Without reducing the generality, functions $\left\{h_{n}\right\}_{n=1, \ldots, M}$ are replaced by the following (Billings, 2013)

$$
h_{n}^{\text {sym }}\left(s_{1}, \ldots, s_{n}\right)=\frac{1}{n !} \sum_{\pi} h_{n}\left(s_{\pi(1)}, \ldots, s_{\pi(n)}\right) .
$$

Let $\left\{H_{n}^{d}\right\}_{n=1, \ldots, M}$ denote the discrete GFRF functions of order $n$. Then the following holds (Billings, 2013)

$$
H_{n}^{d}\left(i \omega_{1}^{d}, \ldots, i \omega_{n}^{d}\right)=\sum_{s_{1}=0}^{\infty} \cdots \sum_{s_{n}=0}^{\infty} h_{n}\left(s_{1}, \ldots, s_{n}\right) e^{-i\left(\omega_{1}^{d} s_{1}+\cdots+\omega_{n}^{d} s_{n}\right)},
$$

for $n=1, \ldots, M$. Without reducing the generality, the discrete GFRFs are replaced by the following functions

$$
H_{n}^{d, s y m}\left(i \omega_{1}^{d}, \ldots, i \omega_{n}^{d}\right)=\frac{1}{n !} \sum_{\pi} H_{n}^{d}\left(i \omega_{\pi(1)}^{d}, \ldots, i \omega_{\pi(n)}^{d}\right)
$$

Let $U^{d}\left(i \omega^{d}\right)$ denote the Fourier transform of the discrete sequence $\{u(k)\}_{k=1, \ldots, N}$, which satisfies

$$
U^{d}\left(i \omega^{d}\right)=\sum_{k=1}^{N} u(k) e^{-i \omega^{d} k}
$$


The discrete output function $y$ satisfies (5.8), (5.9)

$$
y(k)=\sum_{n=1}^{M} \frac{1}{(2 \pi)^{n}} \int_{-\pi}^{\pi} \ldots \int_{-\pi}^{\pi} H_{n}^{d}\left(i \omega_{1}^{d}, \ldots, i \omega_{n}^{d}\right) \prod_{s=1}^{n} U^{d}\left(i \omega_{s}^{d}\right) e^{i\left(\omega_{1}^{d}+\cdots+\omega_{n}^{d}\right) k} d \omega_{s}^{d} .
$$

Under the assumption that sequences $\{u(k)\}_{k=1, \ldots, N},\{y(k)\}_{k=1, \ldots, N}$ represent the uniform samples of continuous functions $u(t), y(t)$, respectively, the following holds true (Billings, 2013)

$$
U^{d}\left(i \omega^{d}\right)=\frac{U(i \omega)}{T_{S}}, Y^{d}\left(i \omega^{d}\right)=\frac{Y(i \omega)}{T_{S}}
$$

where $T_{s}$ denotes the sampling period and $\omega^{d}=T_{s} \omega$. The corresponding discrete and continuous GFRFs satisfy the following

$$
H_{n}\left(i \omega_{1}, \ldots, i \omega_{n}\right)=H_{n}^{d}\left(i \omega_{1}^{d}, \ldots, i \omega_{n}^{d}\right), n=1, \ldots, N
$$

where $\omega_{s}^{d}=T_{s} \omega_{s}, s=1, \ldots, n$.

\subsection{A new method for the identification of [Nonlinear Filter]- [Ideal IF] circuits}

\subsubsection{Problem statement}

The proposed circuit consists of a nonlinear filter connected in series with an IF neuron, as depicted in Figure 5.6.

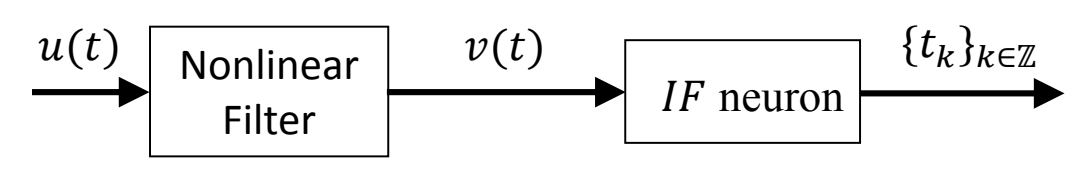

Figure 5.6: The structure of the circuit proposed for identification.

The nonlinear filter is described by the following equations

$$
\left\{\begin{array}{l}
x^{\prime}(t)=f(x(t), u(t)), \\
v(t)=h(x(t), u(t))
\end{array}\right.
$$

where $f: \mathbb{R}^{n} \times \mathbb{R} \rightarrow \mathbb{R}^{n}$ and $h: \mathbb{R}^{n} \times \mathbb{R} \rightarrow \mathbb{R}$ are nonlinear functions, $u$ and $v$ are the input and output, respectively, and $x: \mathbb{R} \rightarrow \mathbb{R}^{n}$ is the state vector function. Let $x_{0}$ be the initial condition of system (5.10). 
The output $v$ of the system is encoded by an ideal IF neuron, described by the $t$ transform equation (2.3). Signal $v$ can be perfectly reconstructed provided that $v \in P W_{\Omega}$ and $\frac{b}{\delta}>\frac{\Omega}{\pi}$ (Lazar and Pnevmatikakis, 2011).

In the following it is assumed that the filter is BIBO-stable and that, for any $\bar{u} \in \mathbb{R}$, the response $v$ of the filter given input $u(t)=\bar{u}, \forall t \in \mathbb{R}$, converges to a steady state value $v_{0}$, i.e. $\exists \lim _{t \rightarrow \infty} v(t)=v_{0}$. In other words, this assumes that $x_{0}$ belongs to the region of attraction of an asymptotically stable equilibrium point. This is a reasonable assumption for the model of a sensory system, which shows adaptation, and thus its output stabilizes to a constant value in the presence of a prolonged constant stimulus (Smith, 2008). Furthermore it is assumed that $v_{0}$ satisfies $\left|v_{0}\right|<b$.

The assumptions above are very easy to verify in practice, as they are equivalent to checking that the length of the inter spike intervals converges, i.e., $\exists \lim _{k \rightarrow \infty}\left(t_{k+1}-t_{k}\right)$. Moreover, the circuit generates no spike times if $\left|v_{0}\right| \geq b$.

Let $I F_{\{C, \delta, b\}}$ denote an ideal IF neuron with parameters $C, \delta, b$. In the following, it will be shown that the identification problem is simplified significantly by estimating the parameters of a circuit with only one spiking neuron parameter, equivalent to the one in Figure 5.6. The following lemma is an important result for designing the equivalent circuit.

Lemma 5.1. Let $\left\{t_{k}\right\}_{k \in \mathbb{Z}}$ be the sequence of spike times generated by neuron $I F_{\{C, \delta, b\}}$ given input $v$. Let $v_{0} \in \mathbb{R}$ such that $\left|v_{0}\right|<b$. Then the following is an equivalent expression for the t-transform of $I F_{\{C, \delta, b\}}$

$$
\int_{t_{k}}^{t_{k+1}} v_{b}(\tau) d \tau=\bar{\delta}_{b}-\left(t_{k+1}-t_{k}\right), \forall k \in \mathbb{Z},
$$

where $\bar{\delta}_{b} \triangleq \frac{\bar{\delta}}{b+v_{0}}$ and $v_{b} \triangleq \frac{v-v_{0}}{b+v_{0}}$.

Proof. The $t$-transform of $I F_{\{C, \delta, b\}}$ satisfies

$$
\begin{gathered}
\int_{t_{k}}^{t_{k+1}} v(\tau) d \tau=\bar{\delta}-b\left(t_{k+1}-t_{k}\right)=\bar{\delta}-\left(b+v_{0}\right)\left(t_{k+1}-t_{k}\right)+v_{0}\left(t_{k+1}-t_{k}\right) \\
\Leftrightarrow \int_{t_{k}}^{t_{k+1}}\left(v(\tau)-v_{0}\right) d \tau=\bar{\delta}-\left(b+v_{0}\right)\left(t_{k+1}-t_{k}\right) .
\end{gathered}
$$

The required result follows after dividing both sides of (5.12) by $\left(b+v_{0}\right)$.

It follows that equation (5.11) is the $t$-transform of $I F_{\left\{1, \bar{\delta}_{b}, 1\right\}}$ and that the two circuits depicted in Figure 5.7 are input-output equivalent, i.e., they generate the same spike train $\left\{t_{k}\right\}_{k \in \mathbb{Z}}$ when presented with the same input $u$ (Lazar and Slutskiy, 2010).

Let $\left\{t_{k}^{\bar{u}}\right\}_{k \in \mathbb{Z}}$ denote the sequence of spike times generated by circuit $\left(a_{2}\right)$ given input 


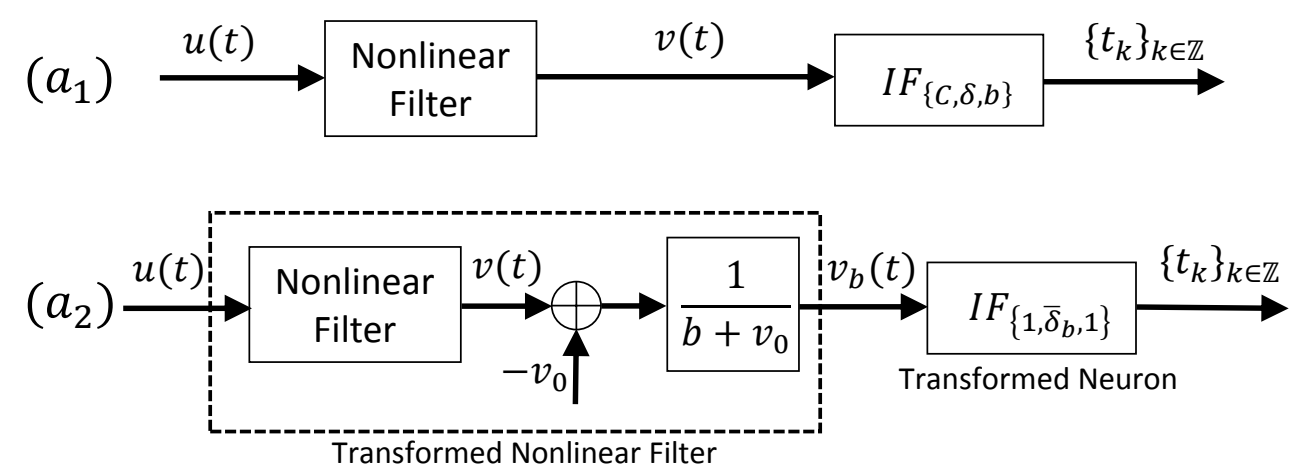

Figure 5.7: Original $\left(a_{1}\right)$ and equivalent $\left(a_{2}\right)$ neural circuit.

$u(t)=\bar{u}, \forall t \in \mathbb{R}$. It follows that $\lim _{t \rightarrow \infty} v_{b}(t)=v_{0}$ and thus (5.11)

$$
\lim _{k \rightarrow \infty}\left(t_{k+1}^{\bar{u}}-t_{k}^{\bar{u}}\right)=\bar{\delta}_{b}
$$

The transformed filter is then identified from function $u$ and the reconstructed input of the IF neuron using the NARMAX system identification methodology. Specifically, the aim is to identify the discrete input-output model (Leontaritis and Billings, 1985a, Leontaritis et al., 1981)

$v_{b}(t)=F\left(v_{b}(t-1), \ldots, v_{b}\left(t-n_{y}\right), u(t-1), \ldots, u\left(t-n_{u}\right), e(t-1), \ldots, e\left(t-n_{e}\right)\right)+e(t)$,

where $e$ is the noise variable, $n_{u} n_{y}$ and $n_{e}$ are the maximum input, output and noise lags, respectively, and $F$ is a polynomial function with expression

$$
F(x(t))=\sum_{j=1}^{m} \theta_{j} p_{j}(x(t)),
$$

where $x(t)=\left[v_{b}(t-1), \ldots, v_{b}\left(t-n_{y}\right), u(t-1), \ldots, u\left(t-n_{u}\right), e(t-1), \ldots, e\left(t-n_{e}\right)\right]$, $p_{j}$ is a monomial of degree $d \leq l$, and $\theta_{j} \in \mathbb{R}, \forall j=1, \ldots, M$. Coefficients $\left\{\theta_{j}\right\}_{j=1, \ldots, m}$ are calculated using the OFR algorithm, presented in Section 5.2.

\subsubsection{Numerical study}

The circuit comprises a nonlinear filter in cascade with an ideal IF neuron. The filter is represented as the quadratic nonlinear system

$$
v^{\prime \prime}+\alpha v^{\prime}+\beta v+\gamma v^{2}=u, \alpha=0.2, \beta=1, \gamma=0.1
$$


System (5.13) can be represented as a Volterra series. The generalized frequency response functions (GFRFs) associated with this system, depicted in Figure 5.8, have the following expressions (Li and Billings, 2011)

$$
H_{1}(i \omega)=\frac{1}{-\omega^{2}+i \alpha \omega s+\beta}
$$

$H_{2}\left(i \omega_{1}, i \omega_{2}\right)=-\gamma H_{1}\left(i \omega_{1}\right) H_{1}\left(i \omega_{2}\right) H_{1}\left(i \omega_{1}+i \omega_{2}\right)$.
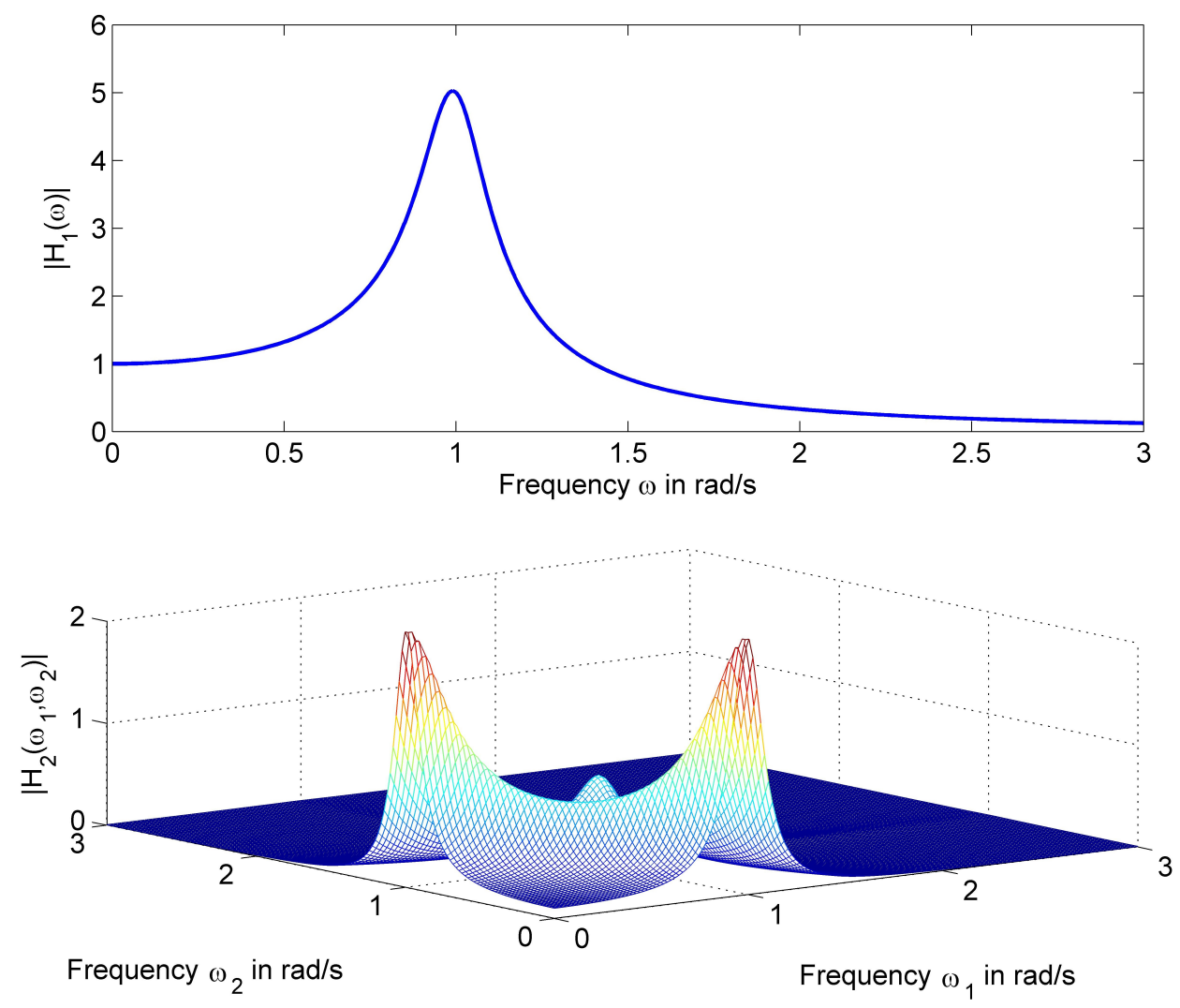

Figure 5.8: The generalised frequency response functions associated with system (5.13).

The circuit responded with uniformly spaced spike train $\left\{t_{k}^{0}\right\}_{k=1,997}$ when excited with input $u=0$. Parameter $\bar{\delta}_{b}$ was estimated as $\bar{\delta}_{b}^{*}=t_{997}^{0}-t_{996}^{0}=0.2$.

Two inputs $u_{t r}, u_{v a l}$ were generated as white Gaussian noise filtered to $\Omega=3 \mathrm{rad} / \mathrm{s}$, sampled with period $\varepsilon_{1}=10^{-3} \mathrm{~s}$ and duration $180 \mathrm{~s}$. System 5.13 responded with signals $v_{t r}, v_{v a l}$ when excited with inputs $u_{t r}, u_{v a l}$, respectively. Functions $u_{t r}, u_{v a l}, v_{t r}, v_{v a l}$ are depicted in Figure 5.9.

The signals $v_{t r}, v_{v a l}$ were encoded with an ideal IF neuron with parameters $b=15, \delta=$ 


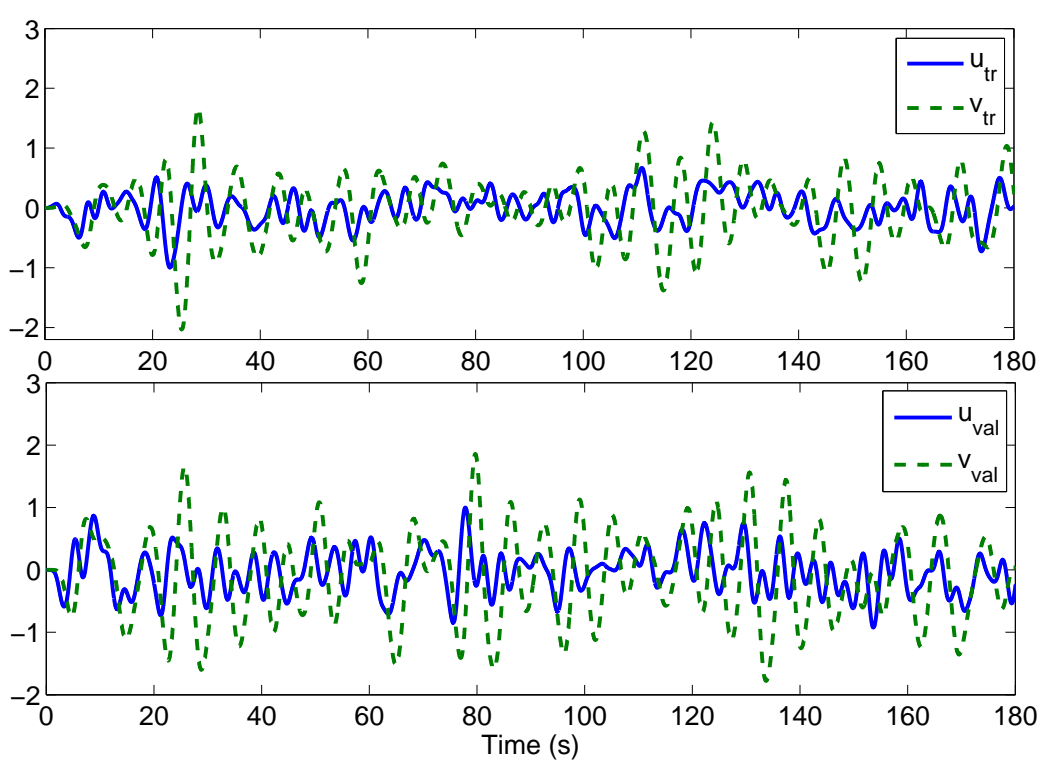

Figure 5.9: Input functions $u_{t r}, u_{v a l}$ and the corresponding output responses $v_{t r}, v_{v a l}$.

3 , and $C=1$ into sequences $\left\{t_{k}^{t r}\right\}_{k=1, \ldots, 1000}$ and $\left\{t_{k}^{v a l}\right\}_{k=1, \ldots, 995}$, respectively.

Let $v_{t r}^{*}$ and $v_{v a l}^{*}$ denote the reconstructed neuron inputs from sequences $\left\{t_{k}^{t r}\right\}_{k=1, \ldots, 1000}$ and $\left\{t_{k}^{v a l}\right\}_{k=1, \ldots, 995}$, with estimated parameter $\bar{\delta}_{b}^{*}$ and $b=C=1$.. The training $\left\{u_{t r}, v_{t r}^{*}\right\}$ and validation data sets $\left\{u_{t r}, v_{t r}^{*}\right\}$ was then resampled with period $\varepsilon_{2}=3 \cdot 10^{-1} \mathrm{~s}$.

A NARX model was identified using the OFR algorithm with input $u_{t r}$ and output $v_{t r}^{*}$. The number of maximum lags was selected as $n_{y}=6$ and $n_{u}=7$ for input and output, respectively. The model was validated with $u_{v a l}$ and $v_{v a l}^{*}$, by computing the normalized mean squared error (NMSE)

$$
N M S E=\frac{\sum_{k=1}^{K}\left(v^{*}(k \varepsilon)-v(k \varepsilon)\right)^{2}}{\sum_{k=1}^{K}(\bar{v}-v(k \varepsilon))^{2}},
$$

where $\bar{v}=\frac{1}{K} \sum_{k=1}^{K} v(k \varepsilon)$ and $K=2 T / \varepsilon$. The following normalised errors were computed to evaluate the performance of the identified models over the training and validation data sets, respectively

$$
N M S E_{t r}=3.9 \cdot 10^{-3}, N M S E_{\text {val }}=4.1 \cdot 10^{-3} .
$$

Let $v_{v a l}^{* *}$ be the response of the fitted model excited with input $u_{v a l}$. The validation error $E$, defined as $E \triangleq\left|v_{v a l}^{* *}-v_{\text {val }}^{*}\right|$, is depicted in Figure 5.10.

To ensure that identified model is an adequate representation of (5.13), the GFRFs of the identified model were calculated analytically and compared with the GFRFs of the original 


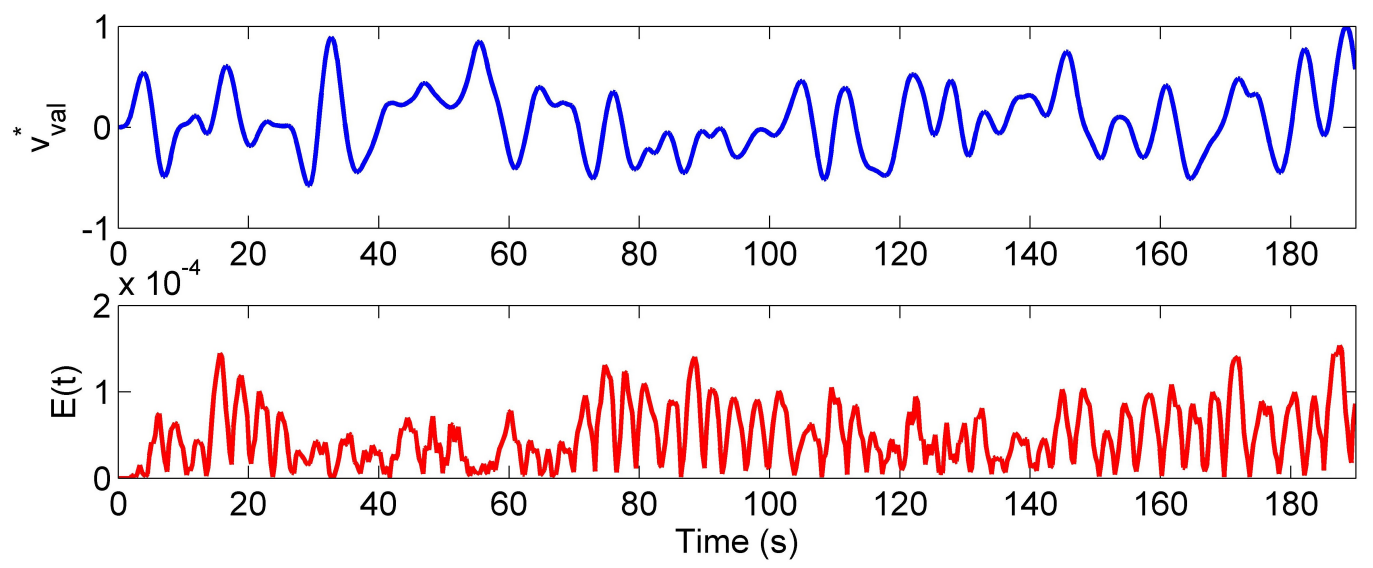

Figure 5.10: Function $v_{v a l}^{*}$ (up) and validation error $E$ (down).

system. It is well known that for a given input-output configuration, the GFRFs are invariant to a change in the representation, i.e., equivalent representations will have the same GFRFs (Billings, 2013).

Let $H_{1}^{*}$ and $H_{2}^{*}$ denote the GFRFs of the identified model of first and second order, respectively. To evaluate to what extent the identified model has captured the dynamics of the original filter, the errors between functions $H_{1}, H_{2}$ and $b \cdot H_{1}^{*}, b \cdot H_{2}^{*}$ were computed. These are depicted in Figure 5.11.

\subsection{A new methodology for the identification of [Linear Filter]- [Leaky IF] circuits}

\subsubsection{Problem statement}

The filter is assumed to be linear with impulse response function $g$ and BIBO-stable. The filter gain $K$ is defined as $K=\lim _{s \rightarrow \infty} G(s)$, where $G$ denotes the Laplace transform of $g$. Let $T_{S}$ denote the settling time of the system.

The filter is connected in series with a leaky IF (LIF) neuron with zero initial condition, described by the $t$ - transform (Lazar, 2005)

$$
\int_{t_{k}}^{t_{k+1}} v(\tau) e^{-\frac{t_{k+1}-\tau}{R C}} d \tau=C(\delta-b R)+b R C \cdot e^{-\frac{t_{k+1}-t_{k}}{R C}}, \forall k \in \mathbb{Z},
$$

where $v$ is the filter output, $b$ is the bias, $\left\{t_{k}\right\}_{k \in \mathbb{Z}}$ are the spike times, and $R$ and $C$ are the filter parameters. 

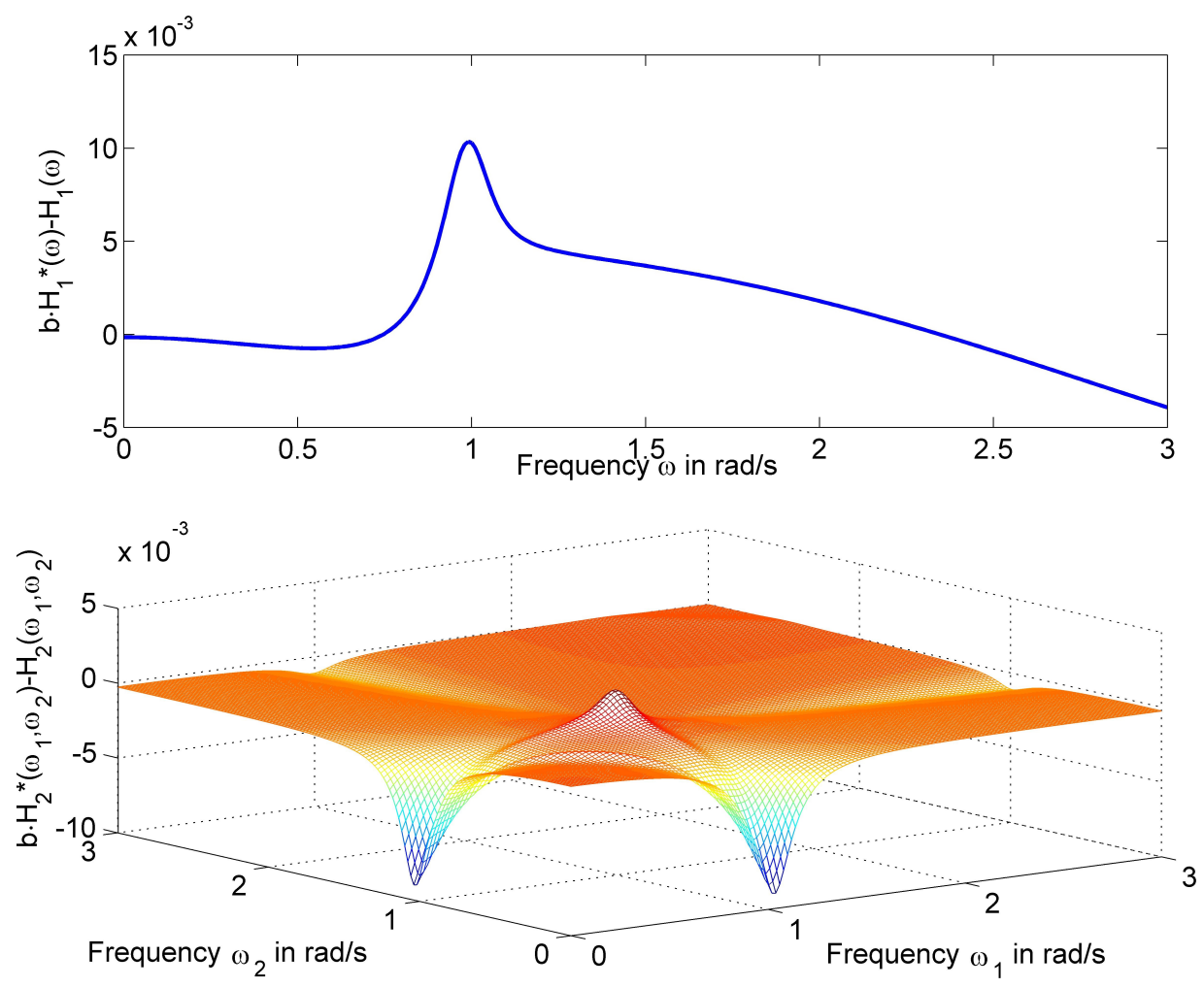

Figure 5.11: The error functions between the original GFRFs $H_{1}, H_{2}$ and the ones computed from the NARX model scaled by $b$, namely $b H_{1}^{*}, b H_{2}^{*}$.

Lazar (2005) has proven that neuron input $v$ can be recovered from spike times $\left\{t_{k}\right\}_{k \in \mathbb{Z}}$ provided that it is bandlimited to $\Omega \mathrm{rad} / \mathrm{s}$, it is bounded $|v| \leq c$ and the following two conditions are satisfied

$$
\begin{gathered}
R C \cdot \ln \left(1-\frac{\delta}{\delta-(b-c) R}\right) \frac{\Omega}{\pi}<\frac{1-\epsilon}{1+\epsilon}, \\
c<b-\frac{\delta}{R^{\prime}}
\end{gathered}
$$

where $\varepsilon=\frac{\delta}{(b-c) R}$.

Let $\operatorname{LIF}_{\{R, C, \delta, b\}}$ denote a LIF neuron with parameters $\{R, C, \delta, b\}$. The $t$ - transform (5.15) is equivalent to

$$
\int_{t_{k}}^{t_{k+1}} v_{b}(\tau) e^{-\frac{t_{k+1}-\tau}{R C}} d \tau=\bar{\delta}_{b}-R C+R C \cdot e^{-\frac{t_{k+1}-t_{k}}{R C}}, \forall k \in \mathbb{Z},
$$


where $\bar{\delta}_{b} \triangleq \bar{\delta} / b$ and $v_{b} \triangleq v / b$. Moreover, $v_{b}$ satisfies (5.17)

$$
\left|v_{b}(t)\right| \leq \frac{c}{b}<1-\frac{\bar{\delta}_{b}}{R C}, \forall t \in \mathbb{R} .
$$

Therefore the two circuits depicted in Figure 5.12 are input-output equivalent.

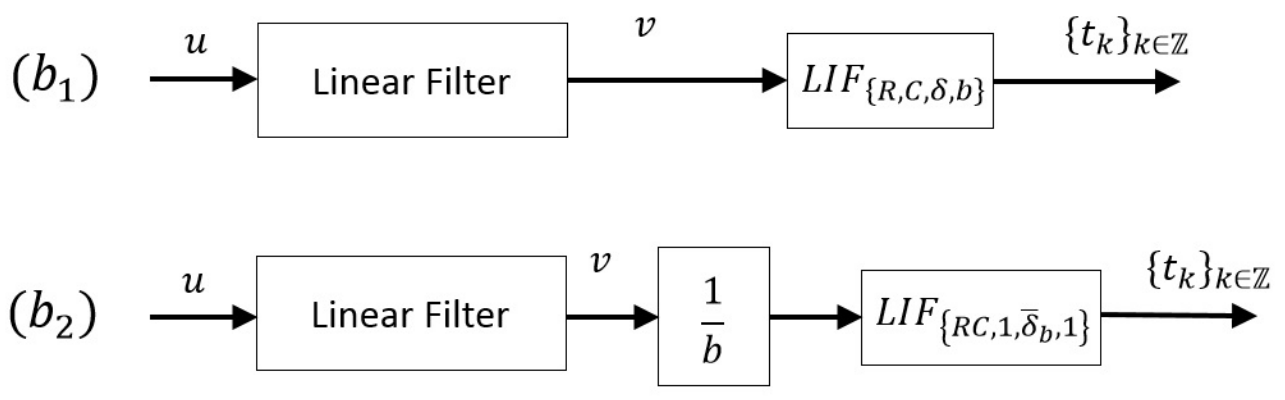

Figure 5.12: Original $\left(b_{1}\right)$ and equivalent $\left(b_{2}\right)$ neural circuit.

The proposed identification methodology is designed for circuit $\left(b_{2}\right)$. This consists of two distinct steps, presented as follows.

\section{Identification of the LIF neuron parameters}

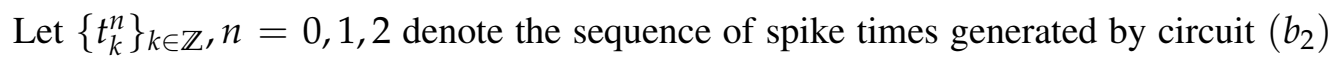
given the three constant inputs $u_{0}(t)=0, u_{1}(t)=-\bar{u}$, and $u_{2}(t)=\bar{u}, \forall t \in \mathbb{R}$, respectively. Let sequences $\left\{I S I_{n, k}\right\}_{k \in \mathbb{Z}}, I S I_{n}, n=0,1,2$ be defined as

$$
\begin{gathered}
I S I_{n, k}=t_{k+1}^{n}-t_{k}^{n}, \forall k \in \mathbb{Z}, \\
I S I_{n} \triangleq \lim _{k \rightarrow \infty} I S I_{n, k}, n=0,1,2 .
\end{gathered}
$$

Moreover, $\lim _{t \rightarrow \infty} v_{b}(t)=\lim _{t \rightarrow \infty} v(t) / b=K / b \lim _{t \rightarrow \infty} u(t)$, and equations (5.15) are equivalent to

$$
R C\left[1-e^{-\frac{I S I_{n}}{R C}}\right]\left(K_{b} u_{n}+1\right)=\bar{\delta}_{b}, n=0,1,2,
$$

where $K_{b} \triangleq \frac{K}{b}$. It follows that

$$
I S I_{n}=-R C \ln \left[1-\frac{\bar{\delta}_{b}}{R C\left(K_{b} u_{n}+1\right)}\right], \forall n=0,1,2 .
$$

The expression of $I S I_{n}$ in (5.19) is decreasing as a function of $u_{n}$. Thus values of the inter-spike intervals satisfy $0<I S I_{2}<I S I_{0}<I S I_{1}$. 
The following lemma derives three equations equivalent to (5.18) that enable computing the LIF neuron parameters.

Lemma 5.2. Equations (5.18) are equivalent to

$$
\begin{gathered}
\bar{\delta}_{b}=R C\left(1-e^{-\frac{I S I_{0}}{R C}}\right), \\
K_{b}=\frac{1}{u_{1}}\left[\frac{1-e^{-\frac{I S I_{0}}{R C}}}{1-e^{-\frac{I S I_{1}}{R C}}}-1\right], \\
\frac{1-e^{-\frac{I S I_{1}}{R C}}}{1-2 e^{-\frac{I S I_{1}}{R C}}+e^{-\frac{I S I_{0}}{R C}}}=\frac{1-e^{-\frac{I S I_{2}}{R C}}}{1-e^{-\frac{I S I_{0}}{R C}}} .
\end{gathered}
$$

Proof. Equation (5.20) follows directly from (5.18) for $n=0$. Equations (5.21) and (5.22) are derived from (5.18) for $n=1,2$, by substituting $\bar{\delta}_{b}$ and then $K_{b}$ with its corresponding expression.

The following theorem is the main result of this section, which proves that the LIF neuron parameters $\left\{\bar{\delta}_{b}, R C\right\}$ can be uniquely determined from input-output measurements $\left\{u_{n}, I S I_{n}\right\}_{n=0,1,2}$ of circuit $\left(b_{2}\right)$.

Theorem 5.2. Let $\left\{I S I_{n}\right\}_{n=0,1,2}$ be the inter-spike interval values generated by neural circuit $\left(b_{2}\right)$ at steady state, when presented with constant inputs $u_{0}(t)=0, u_{1}(t)=-\bar{u}$, $u_{2}(t)=\bar{u}, \forall t>0$, respectively, such that

$$
\bar{u} \in] 0, \bar{u}_{M}[
$$

where $\bar{u}_{M}=\frac{R C-\bar{\delta}_{b}}{K_{b} \cdot R C}$.

Let $\bar{f}: \mathbb{R}_{+}^{*} \rightarrow \mathbb{R}$ be defined by

$$
\bar{f}(x)=\frac{1-e^{-\frac{I S I_{1}}{x}}}{1-2 e^{-\frac{I S I_{1}}{x}}+e^{-\frac{I S I_{0}}{x}}}-\frac{1-e^{-\frac{I S I_{2}}{x}}}{1-e^{-\frac{I S I_{0}}{x}}}, \forall x>0 .
$$

Then equation $\bar{f}(x)=0$ has a unique solution $x_{0}=R C$. Moreover,

$$
\operatorname{sgn}(\bar{f}(x))=\operatorname{sgn}(R C-x)
$$

where sgn: $\mathbb{R} \rightarrow\{-1,0,1\}$ denotes the sign function.

Proof. See Appendix B.

Remark 2. If $\bar{u} \geq \frac{R C-\bar{\delta}_{b}}{K_{b} \cdot R C}$, circuit $\left(b_{2}\right)$ generates no spike times when excited with input $u_{2}(t)=-\bar{u}$ (5.19). Therefore, it is always possible to find a suitable value for $\bar{u}>0$ that satisfies (5.23), without any knowledge of the circuit parameters. 
Equation $\bar{f}(x)=0$ is solved using the bisection method, which calculates iteratively sequence $\boldsymbol{x}_{n} \in \mathbb{R}^{2}, \boldsymbol{x}_{n} \triangleq\left[x_{n, 1}, x_{n, 2}\right], n \geq 0$, where

$$
\boldsymbol{x}_{n+1}= \begin{cases}{\left[\frac{x_{n, 1}+x_{n, 2}}{2}, x_{n, 2}\right],} & \bar{f}\left(\frac{x_{n, 1}+x_{n, 2}}{2}\right)>0, \\ {\left[x_{n, 1}, \frac{x_{n, 1}+x_{n, 2}}{2}\right],} & \bar{f}\left(\frac{x_{n, 1}+x_{n, 2}}{2}\right)<0,\end{cases}
$$

where $x_{0,1}, x_{0,2} \in \mathbb{R}$ such that $x_{0,1}<x_{0,2}$ and $\bar{f}\left(x_{0,1}\right) \cdot \bar{f}\left(x_{0,2}\right)<0$. Due to (5.25), it follows that

$$
R C \in\left[x_{n, 1}, x_{n, 2}\right], \forall n \in \mathbb{N},
$$

and thus

$$
\lim _{n \rightarrow \infty} x_{n}=[R C, R C] .
$$

In practice, iterations (5.26) are calculated until the stop criterion $\left|x_{n, 2}-x_{n, 1}\right|<t o l$ is satisfied, where tol represents a tolerance parameter selected by the user. Once $R C$ is estimated with the desired accuracy, parameters $K_{b}$ and $\bar{\delta}_{b}$ can subsequently be determined with Lemma 5.2.

\section{Identification of the filter}

The filter scaled by $1 / b$ can be identified from an input $u$ and the corresponding signal $v_{b}$, reconstructed with the identified parameters $R C, \bar{\delta}_{b}$ and $K_{b}$ using any of the existent techniques. Here the ARX (AutoRegressive with eXogenous inputs) representation of the scaled filter is derived by using the orthogonal forward selection algorithm (Billings, 2013).

The filter scaled by $1 / b$ is then identified from function $u$ and the reconstructed input of the IF neuron using the ARMAX system identification methodology. Specifically the aim is to identify the discrete input-output model (Billings, 2013)

$$
\begin{aligned}
v(t)+a_{1} y(t-1)+\cdots+a_{n_{y}} y\left(t-n_{y}\right) & =b_{1} u(t-1)+\ldots, b_{n_{u}} u\left(t-n_{u}\right) \\
& +e(t)+c_{1} e(t-1)+\cdots+c_{n_{e}} e\left(t-n_{e}\right),
\end{aligned}
$$

where $e$ is the noise variable, $n_{u} n_{y}$ and $n_{e}$ are the maximum input, output and noise lags,

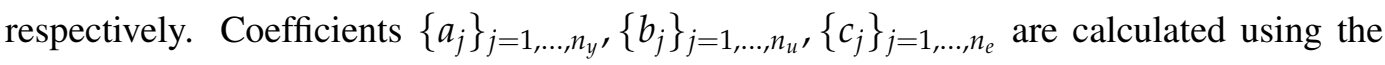
OFR algorithm, presented in Section 5.2.

\subsubsection{Numerical study}

The linear filter has the following transfer function

$$
G(s)=\frac{K}{\tau^{2} s^{2}+2 \zeta \tau s+1}
$$


where $\tau=0.1, \zeta=0.2, K=0.8$. The filter is connected in cascade with a LIF neuron with parameters $R=2 \cdot 10^{-2}, C=1, \delta=0.02$ and $b=4$.

The LIF neuron parameters were estimated by presenting the circuit with three constant inputs $u_{0}=0, u_{1}=-2$ and $u_{2}=2$, generated on time interval $[0,4 \mathrm{~s}]$ with sampling time $\varepsilon_{1}=10^{-7}$. The circuit responded with output spike time sequences $\left\{t_{k}^{n}\right\}_{k=1, \ldots, K_{n}+1, n=}$ $0,1,2, K_{0}=695, K_{1}=374, K_{2}=1013$. The corresponding sequences of inter-spike intervals $\left\{s_{k}^{n}\right\}_{k=1, \ldots, K_{n}}$, where $s_{k}^{n}=t_{k+1}^{n}-t_{k}^{n}, n=0,1,2$, are depicted in Figure 5.13.

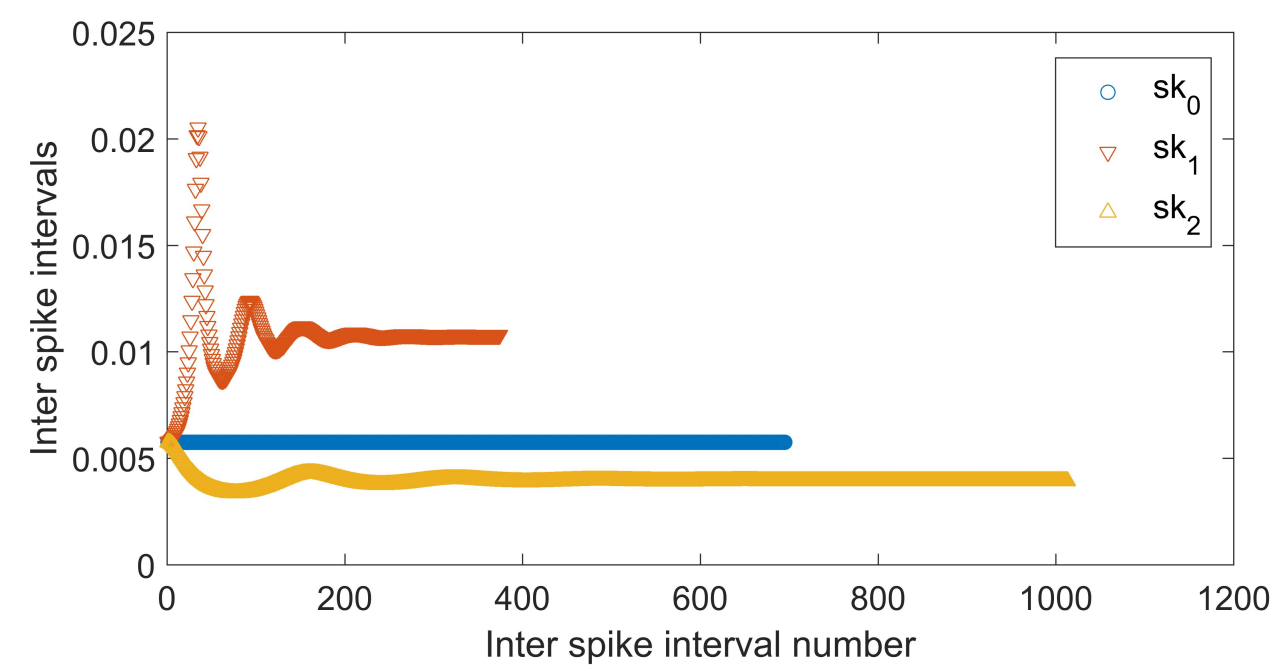

Figure 5.13: The output of circuit $\left(b_{2}\right)$ for inputs $u_{0}, u_{1}$ and $u_{2}$.

Values $I S I_{n}, n=0,1,2$, were computed as

$$
I S I_{n}=\frac{1}{10} \sum_{k=0}^{9} s_{K_{n}-k}^{n}, n=0,1,2 .
$$

Function $\bar{f}(x), x \in] 0,1]$, was computed and plotted in Figure 5.14 together with the real value for parameter $R C$.

Parameter $R C$ was estimated iteratively as $R C^{*}=2.002 \cdot 10^{-2}$ with (5.26) and tolerance $t o l=0.005 \cdot 10^{-2}$. Parameter $\bar{\delta}_{b}$ was estimated as

$$
\bar{\delta}_{b}^{*}=R C^{*}\left(1-e^{-\frac{I S I_{0}}{R C^{*}}}\right)=5.006 \cdot 10^{-3} .
$$

Two inputs $u_{t r}, u_{v a l}$ were generated as white Gaussian noise bandlimited to $50 \mathrm{rad} / \mathrm{s}$, with sampling time $\varepsilon_{2}=10^{-3} \mathrm{~s}$ and duration $5 \mathrm{~s}$. The responses $v_{t r}$ and $v_{v a l}$ of system (5.27) to inputs $u_{t r}$ and $u_{v a l}$ were encoded with the LIF neuron into spike time sequences

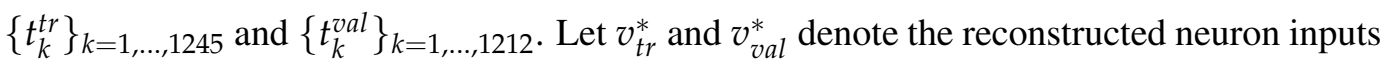




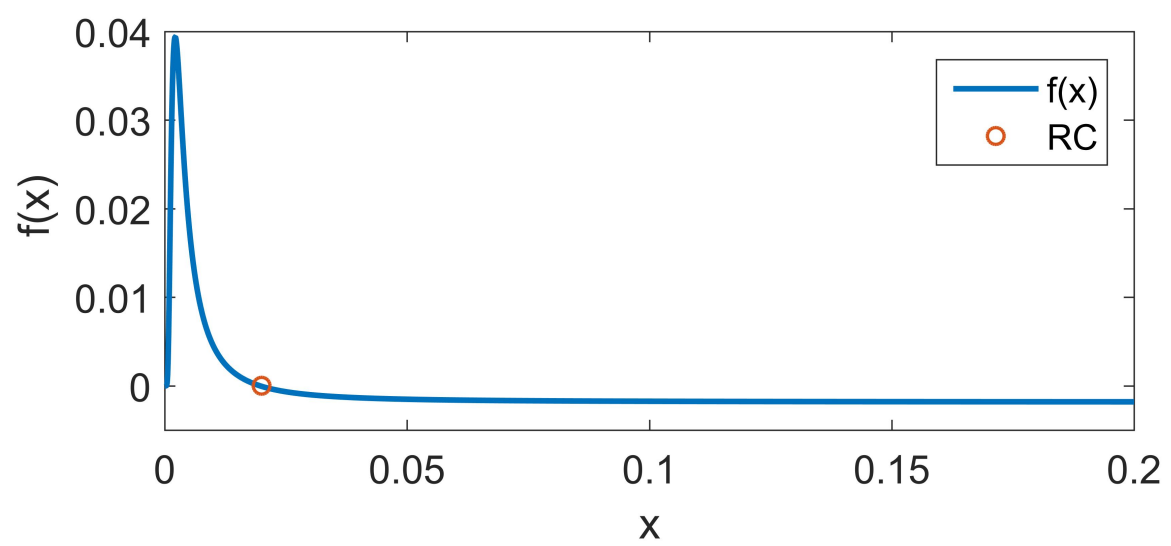

Figure 5.14: Function $\bar{f}(x)$ and the real value of parameter $R C$.

from sequences $\left\{t_{k}^{t r}\right\}_{k=1, \ldots, 1245}$ and $\left\{t_{k}^{v a l}\right\}_{k=1, \ldots, 1212}$, with estimated parameter $\bar{\delta}_{b}^{*}, R C^{*}$ and $b=C=1$.

An ARX model was identified using the OFR algorithm with input $u_{t r}$ and output $v_{t r}^{*}$. The number of maximum lags was selected as $n_{y}=6$ and $n_{u}=7$ for input and output, respectively. The model was validated with $u_{v a l}$ and output $v_{v a l}^{*}$, reconstructed from $\left\{t_{k}^{v a l}\right\}$.

The NMSE (5.14) was computed for training and validation as

$$
N M S E_{t r}=1.06 \cdot 10^{-5}, N M S E_{\text {val }}=2.84 \cdot 10^{-5}
$$

To ensure that identified model is an adequate representation of (5.27), the magnitude curve of the identified model was calculated analytically and compared with the one from the original system. The magnitude plot of the original system is depicted in Figure 5.15. The errors between the original and identified magnitude plot scaled by $b$ are depicted in Figure 5.16.

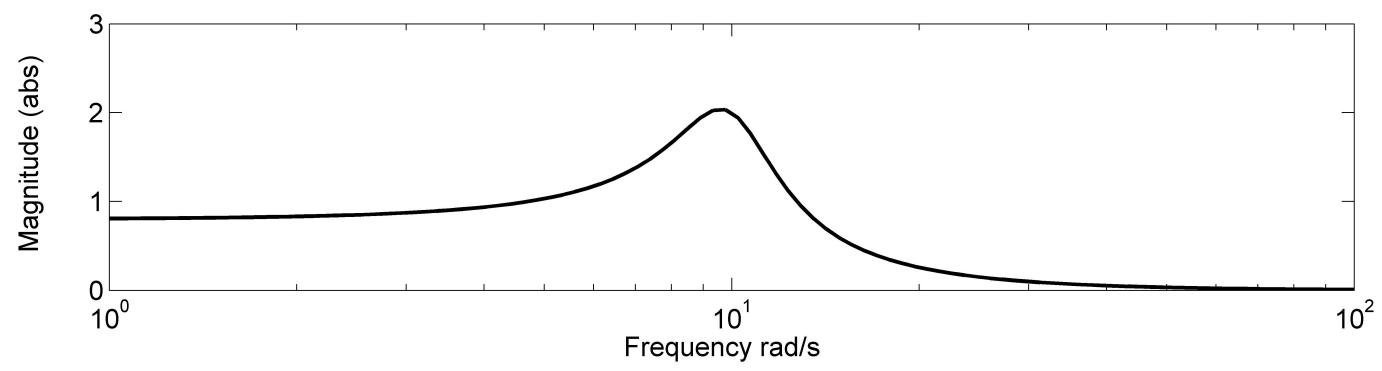

Figure 5.15: Magnitude plot of system (5.27). 


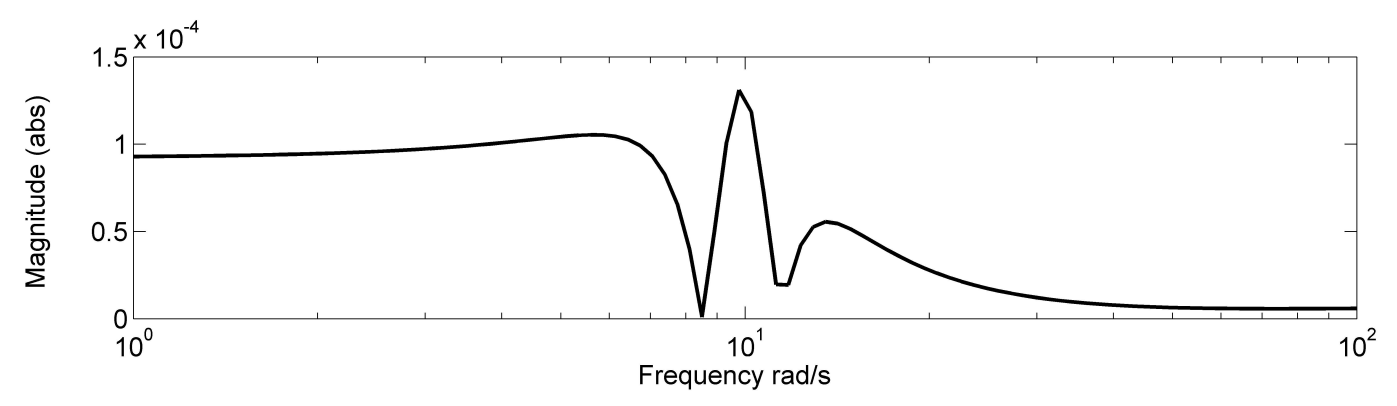

Figure 5.16: The error between the original and the identified magnitude curve scaled by $b$.

\subsection{Conclusions}

This chapter reviewed the identification methods for a [Filter]-[Spiking Neuron] circuit with single and multiple inputs, and for various filter and spiking neuron models. The existent methodologies involve redefining the filter identification problem as one of stimulus reconstruction.

The assumptions of the existent identification methods can in some cases be considered restrictive. Specifically, the identification of a [Nonlinear Filter]-[Ideal IF] circuit was performed from input-output data under the assumption that the filter can be represented as a Volterra series. For different spiking neuron models, the existing identification methods assume that the neuron model is known, or that its input-output measurements are available.

Two new system identification methodologies have been proposed for circuits consisting of filters in cascade with spiking neurons. Both methods assume that the circuit is unknown and that the input of the neuron (i.e. the output of the filter) is not available for measurement.

The first methodology was proposed for the identification of a [Nonlinear Filter]-[Ideal IF] circuit. The circuit was identified directly from the simulation data using the NARMAX methodology to infer the structure and parameters of the filter.

The second methodology was developed for estimating a [Linear Filter]-[Leaky IF] circuit. The leaky IF neuron parameters were estimated with arbitrary precision using specific stimuli sequences. The structure and parameters of the filter were subsequently identified from the reconstructed neuron input using the NARMAX methodology.

Both methods have been tested through numerical simulations, which showed that the identified models are accurate representations of the original circuits. 


\section{Chapter 6}

\section{A new method for implementing linear filters in the spike domain}

Integrated circuits are continuously decreasing in size, and thus the implementation of the corresponding analog-to-digital converters has increasingly higher demands for low power and high precision in amplitude. In this context, temporal encoding devices like time encoding machines offer an alternative to classical analog-to-digital converters, by exchanging the amplitude axis for the time axis (Lazar et al., 2008, Roza, 1997).

An ideal IF neuron has been used in a brain machine interface (BMI) prototype for neural recordings using implantable electrodes called FWIRE (Bashirullah et al., 2007). The prototype encodes the recorded neural information in an asynchronous train of spikes, thus providing high resolution amplitude measurements with great power savings and noise immunity.

TEMs have also been included in a human area network (HAN) biomonitoring prototype. Specifically, the application involves an ASDM circuit that encodes information from sensors and transmits it using the human skin as a channel (Káldy et al., 2007). The paper showed that their proposed prototype offers a reduced level of sensor interference compared to the schemes using amplitude sampling.

One of the shortcomings of the transmission systems with TEMs is that any processing of the recorded signals after they are being encoded, for example filtering or amplifying, involves reconstruction back to amplitude samples, processing, and subsequent encoding, which would negatively affect the low power as well as noise/interference immunity of the encoding. The problem proposed in this chapter is to determine the output of a linear filter in cascade with an ideal IF neuron directly from the filter input encoded with the same IF neuron, without the need to simulate the filter.

Lazar (2006b) has considered the problem of realizing an arbitrary linear filter in the spike domain. The method performs simultaneously the computation of the filter output 
and the reconstruction of the spike train in analog domain.

This chapter develops a direct relationship between the filter input and output spike time sequences. The proposed representation forms the basis for an algorithm to compute the time encoded output directly from the input spike time sequence. The approximation error introduced by the proposed implementation is characterized by deriving an error bound between the real and estimated spike times that is a function of the IF neuron parameters.

The main advantage of the proposed algorithm is that it is significantly faster than the alternative approach, which involves decoding the input time sequence, computing the ana$\log$ output of the linear filter, and finally encoding the resulting output. For this reason, the algorithm is particularly suited for practical implementation of signal processing circuits that operate on time encoded rather than amplitude encoded signals.

This chapter is structured as follows. Section 6.1 introduces the proposed problem. Section 6.2 describes the procedure for computing the output spike times. Numerical results are presented in Section 6.3. Conclusions are in Section 6.4.

\subsection{Problem statement}

Let $g \in L^{1}(\mathbb{R}) \cap C(\mathbb{R}),\|g\|_{L^{1}} \leq 1$ be the impulse response of a linear filter, and let $u \in L^{1}(\mathbb{R}) \cap L^{2}(\mathbb{R}) \cap C(\mathbb{R}),|u(t)| \leq c<b, \forall t \in \mathbb{R}$.

Let $y$ be the output of the linear filter with impulse response function $g$ for a given input $u$, such that

$$
y(t)=\int_{-\infty}^{\infty} u(\tau) g(t-\tau) d \tau
$$

It follows that $y$ satisfies

$$
|y(t)| \leq \int_{-\infty}^{\infty}|u(\tau)| \cdot|g(t-\tau)| d \tau \leq c\|g\|_{L^{1}} \leq c .
$$

Moreover, according to the properties of the convolution operator, $y \in L^{2}(\mathbb{R}) \cap L^{1}(\mathbb{R})$.

The ideal IF neuron is described by its $t$-transform (Lazar and Pnevmatikakis, 2008a):

$$
\int_{t_{k}}^{t_{k+1}} u(t) d t=\bar{\delta}-b\left(t_{k+1}-t_{k}\right), \forall k \in \mathbb{Z}
$$

where $\bar{\delta}=C \delta$, and $\delta, C$, and $b$ are the neuron threshold, integration constant, and bias, respectively. The encoding mechanism of the IF neuron is depicted in Figure 2.1.

Let $\left\{t_{k}^{u}\right\}_{k \in \mathbb{Z}}$ and $\left\{t_{k}^{y}\right\}_{k \in \mathbb{Z}}$ be the spike times triggered by the neuron when presented with inputs $u$ and $y$, respectively. Without reducing the generality, it is assumed that $t_{0}^{u}=$ $t_{0}^{y}=0$.

Signals $u$ and $y$ can be perfectly recovered from $\left\{t_{k}^{u}\right\}_{k \in \mathbb{Z}}$ and $\left\{t_{k}^{y}\right\}_{k \in \mathbb{Z}}$, respectively, provided that $u, y \in P W_{\Omega}$ and the Nyquist-type requirement (2.9) is satisfied. 
For inputs that are not bandlimited, the reconstructed signal is constrained to generate the same spike times as the original stimulus, as well as to minimize a proposed criterion (Lazar and Pnevmatikakis, 2009).

The problem proposed here is to compute $\left\{t_{k}^{y}\right\}_{k \in \mathbb{Z}}$, based only on $\left\{t_{k}^{u}\right\}_{k \in \mathbb{Z}}, g$, and IF neuron model. In other words, the problem that is being addressed is that of implementing a linear filter directly in the spike domain, as depicted in Figure 6.1.

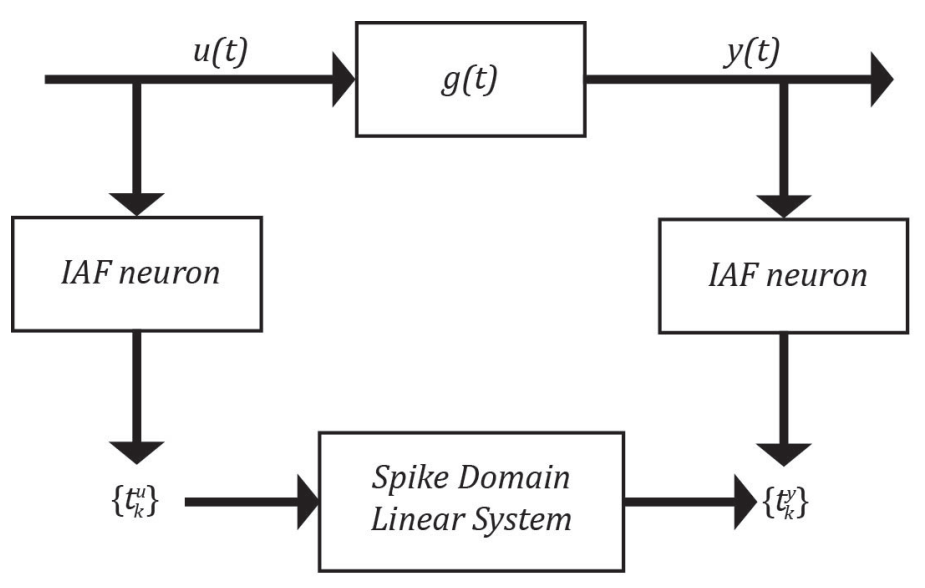

Figure 6.1: Linear filter implementation in the spike domain.

Figure 6.1 illustrates the main idea of this chapter: sequences $\left\{t_{k}^{u}\right\}_{k \in \mathbb{Z}}$ and $\left\{t_{k}^{y}\right\}_{k \in \mathbb{Z}}$, connected indirectly through a linear system with impulse response function $g(t)$, also have an underlying direct connection. The following theoretical results will make an attempt to uncover this connection.

The following functions are defined

$$
\begin{aligned}
& U(t) \triangleq \int_{0}^{t} u(\tau) d \tau \\
& Y(t) \triangleq \int_{0}^{t} y(\tau) d \tau .
\end{aligned}
$$

It follows that $U$ and $Y$ are bounded by $|U(t)| \leq\|u\|_{L^{1}}$ and $|Y(t)| \leq\|y\|_{L^{1}} \leq$ $\|u\|_{L^{1}} \cdot\|g\|_{L^{1}}$, respectively, and 


$$
\begin{aligned}
Y(t) & =\int_{0}^{t} \int_{-\infty}^{\infty} g(\tau) u(s-\tau) d \tau d s \\
& =\int_{-\infty}^{\infty} g(\tau) \int_{0}^{t} u(s-\tau) d s d \tau \\
& =\int_{-\infty}^{\infty} g(\tau)[U(t-\tau)-U(-\tau)] d \tau \\
& =\int_{-\infty}^{\infty} U(\tau)[g(t-\tau)-g(-\tau)] d \tau .
\end{aligned}
$$

It follows that (6.1), (6.2)

$$
\begin{aligned}
& U\left(t_{k}^{u}\right)=k \bar{\delta}-b t_{k}^{u}, \forall k \in \mathbb{Z}, \\
& Y\left(t_{k}^{y}\right)=k \bar{\delta}-b t_{k}^{y}, \forall k \in \mathbb{Z} .
\end{aligned}
$$

\subsection{Direct computation of spike times}

Let $\widetilde{f}_{k}: \mathbb{R} \rightarrow \mathbb{R}$, be a function defined by

$$
\widetilde{f}_{k}(t)=\frac{1}{b}(k \bar{\delta}-\tilde{Y}(t))
$$

where

$$
\widetilde{Y}(t)=\int_{-\infty}^{\infty} I_{1} U(\tau)[g(t-\tau)-g(-\tau)] d \tau,
$$

where $I_{1} U$ is the piecewise constant interpolant to $U$ at points $\left\{t_{k}^{u}\right\}_{k \in \mathbb{Z}}$.

The following theorem proposes a methodology for implementing the spike domain linear system depicted in Figure 6.1. Specifically, the theorem calculates values $\left\{t_{k}^{y}\right\}_{k \in \mathbb{Z}}$ by solving iteratively the following fixed point equation

$$
\widetilde{f}_{k}(t)=t, t \in \mathbb{R}
$$

The existence of the fixed point is proven, and a bound is given for the error between the solution of the equation and real spike time. Moreover, the theorem defines a sequence with arbitrarily chosen initial condition whose limit is in a neighbourhood of the real spike time of radius given by the previously calculated error bound.

Theorem 6.1. Let $u \in L^{1}(\mathbb{R}) \cap L^{2}(\mathbb{R}) \cap C(\mathbb{R}),|u(t)| \leq c<b, U(t)=\int_{0}^{t} u(\tau) d \tau$, $g \in L^{1}(\mathbb{R}) \cap C(\mathbb{R}),\|g\|_{L^{1}} \leq 1$, and $y=u * g$, where $*$ denotes the convolution operator. Let $\left\{t_{k}^{u}\right\}_{k \in \mathbb{Z}}$ and $\left\{t_{k}^{y}\right\}_{k \in \mathbb{Z}}$ be the spike trains triggered by an ideal IF neuron with parameters $b, \bar{\delta}$, given inputs $u$ and $y$, respectively. Then the following hold true 
(a) $\forall k \in \mathbb{Z}, \exists \widetilde{t_{k}^{y}} \in\left[t_{k}^{y}-\frac{E}{b-c}, t_{k}^{y}+\frac{E}{b-c}\right]$ such that $\widetilde{f}_{k}\left(\widetilde{t_{k}^{y}}\right)=\widetilde{t}_{k^{y}}$, where $E=2 \Delta c\|g\|_{L^{1}}, \Delta=$ $\sup _{k \in \mathbb{Z}}\left(t_{k+1}^{u}-t_{k}^{u}\right)$.

(b) $\lim \sup _{m \rightarrow \infty} t_{k, m}^{y} \in\left[t_{k}^{y}-\frac{E}{b-c}, t_{k}^{y}+\frac{E}{b-c}\right]$,

$\liminf _{m \rightarrow \infty} t_{k, m}^{y} \in\left[t_{k}^{y}-\frac{E}{b-c}, t_{k}^{y}+\frac{E}{b-c}\right]$, where $\left\{t_{k, m}^{y}\right\}_{m \in \mathbb{N}}$ satisfies $t_{k, m+1}^{y}=\widetilde{f}_{k}\left(t_{k, m}^{y}\right)$, and $t_{k, 0}^{y}$ is an arbitrary positive number.

Proof. (a) Function $\widetilde{Y}$ satisfies

$$
\begin{aligned}
& |Y(t)-\tilde{Y}(t)| \leq \\
& \leq \int_{-\infty}^{\infty}\left|U(\tau)-I_{1} U(\tau)\right| \cdot|g(t-\tau)-g(-\tau)| d \tau \\
& \leq \Delta \cdot \sup _{\tau \in \mathbb{R}}\left|U^{\prime}(\tau)\right| \cdot \int_{-\infty}^{\infty}|g(t-\tau)-g(-\tau)| d \tau \leq E .
\end{aligned}
$$

Let $f_{k}=\frac{1}{b}(k \bar{\delta}-Y)$. From (6.3), it follows that $\forall k \in \mathbb{Z}, f_{k}\left(t_{k}^{y}\right)=t_{k}^{y}$. The following holds

$$
\left|f_{k}^{\prime}(t)\right|=\left|-\frac{y(t)}{b}\right| \leq \frac{c}{b}
$$

and thus

$$
\forall \zeta \geq 0, \forall t \in\left[t_{k}^{y}-\zeta, t_{k}^{y}+\zeta\right], f_{k}(t) \in\left[t_{k}^{y}-\zeta_{\bar{b}}^{c}, t_{k}^{y}+\zeta_{\bar{b}}^{c}\right]
$$

It follows that $\left|f_{k}(t)-\widetilde{f}_{k}(t)\right| \leq \frac{E}{b}, \forall t \in \mathbb{R}$, and (6.5), (6.6)

$$
\forall \zeta \geq 0, \forall t \in\left[t_{k}^{y}-\zeta, t_{k}^{y}+\zeta\right], \widetilde{f}_{k}(t) \in\left[t_{k}^{y}-\zeta_{\frac{c}{b}}^{c}-\frac{E}{b}, t_{k}^{y}+\zeta_{\frac{c}{b}}^{c}+\frac{E}{b}\right]
$$

such that, for $\zeta=\frac{E}{b-c}$,

$$
\forall t \in\left[t_{k}^{y}-\frac{E}{b-c}, t_{k}^{y}+\frac{E}{b-c}\right], \widetilde{f}_{k}(t) \in\left[t_{k}^{y}-\frac{E}{b-c}, t_{k}^{y}+\frac{E}{b-c}\right]
$$

Given that $\widetilde{f}_{k}$ is continuous, applying Brouwer's fixed point theorem yields the required result (6.8).

(b) By choosing $\zeta=t_{k, 0}^{y}$ in (6.7), it follows that

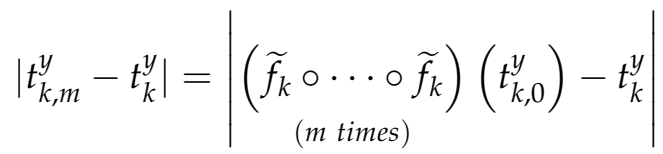

$$
\begin{aligned}
& \leq t_{k, 0}^{y}\left(\frac{c}{b}\right)^{m}+\frac{E}{b} \cdot \sum_{i=1}^{m}\left(\frac{c}{b}\right)^{i-1},
\end{aligned}
$$


where $\circ$ denotes function composition. It follows that

$$
\left|t_{k, m}^{y}-t_{k}^{y}\right| \leq t_{k, 0}^{y}\left(\frac{c}{b}\right)^{m}+\frac{E}{b-c} \cdot\left(1-\left(\frac{c}{b}\right)^{m}\right) .
$$

Equivalently,

$$
\begin{aligned}
& t_{k, m}^{y} \leq t_{k}^{y}+t_{k, 0}^{y}\left(\frac{c}{b}\right)^{m}+\frac{E}{b-c} \cdot\left(1-\left(\frac{c}{b}\right)^{m}\right), \\
& t_{k, m}^{y} \geq t_{k}^{y}-t_{k, 0}^{y}\left(\frac{c}{b}\right)^{m}-\frac{E}{b-c} \cdot\left(1-\left(\frac{c}{b}\right)^{m}\right) .
\end{aligned}
$$

The following holds

$$
\begin{gathered}
\liminf _{m \rightarrow \infty} t_{k, m}^{y} \geq t_{k}^{y}-\frac{E}{b-c} \\
\limsup _{m \rightarrow \infty}^{y} t_{k, m}^{y} \leq t_{k}^{y}+\frac{E}{b-c} .
\end{gathered}
$$

Given that $|u(t)| \leq c$, the following hold true (6.3)

$$
\frac{\bar{\delta}}{b+c} \leq t_{k+1}^{u}-t_{k}^{u} \leq \frac{\bar{\delta}}{b-c}, \forall k \in \mathbb{Z}
$$

An upper bound for the timing error introduced by the proposed representation, which depends only on the model parameters, can be derived from Theorem 6.1 as follows.

$$
\left|\widetilde{t_{k}^{y}}-t_{k}^{y}\right| \leq \frac{2 \Delta c\|g\|_{L^{1}}}{b-c} \leq \frac{2 \bar{\delta} c}{(b-c)^{2}}
$$

The error can be made arbitrarily small by adjusting the IF parameters $\bar{\delta}$ or/and $b$.

An important requirement for the estimated spike train $\left\{\widetilde{t}_{k}^{y}\right\}_{k \in \mathbb{Z}}$ is that it should be strictly increasing, which is not a direct consequence of Theorem 6.1. In the following, a sufficient condition is provided for this requirement to be satisfied.

Sequence $\left\{t_{k}^{y}\right\}_{k \in \mathbb{Z}}$ is strictly increasing, due to the $t$-transform definition. It follows that $\left\{\tilde{t}_{k}^{\breve{y}}\right\}_{k \in \mathbb{Z}}$ is also strictly increasing provided that the following holds (6.10)

$$
\begin{gathered}
\widetilde{t_{k}^{y}<t_{k}^{y}}+\frac{2 \bar{\delta} c}{(b-c)^{2}}<t_{k+1}^{y}-\frac{2 \bar{\delta} c}{(b-c)^{2}}<\widetilde{t_{k+1}^{y}} \\
\Leftrightarrow t_{k+1}^{y}-t_{k}^{y}>\frac{4 \bar{\delta} c}{(b-c)^{2}}, \forall k \in \mathbb{Z} .
\end{gathered}
$$


The following is satisfied

$$
\frac{\bar{\delta}}{b+c} \leq t_{k+1}^{y}-t_{k}^{y} \leq \frac{\bar{\delta}}{b-c}, \forall k \in \mathbb{Z}
$$

The following inequality is a sufficient condition for (6.11) to hold true (6.12)

$$
\frac{4 \bar{\delta} c}{(b-c)^{2}}<\frac{\bar{\delta}}{b+c} \Leftrightarrow b^{2}-3 c^{2}-6 b c>0 \Leftrightarrow\left(\frac{b}{c}\right)^{2}-6 \cdot \frac{b}{c}-3>0 .
$$

The parameters $b$ and $c$ satisfy $b \geq c>0$. Thus (6.13) is equivalent to $\frac{b}{c}>3+2 \sqrt{3}$.

The output spike train $\left\{\widetilde{t}_{k}^{y}\right\}_{k=0, \ldots, K}$ is computed from sequence $\left\{t_{l}^{u}\right\}_{l=0, \ldots, L}$ as follows, where $t_{0}^{u} \leq \widetilde{t_{k}} \leq t_{L}^{u}, \forall k=0, \ldots, K$.

\section{Algorithm 6.1.}

Step 1. Compute $\widetilde{Y}\left(\widetilde{t}_{k-1}^{y}\right)$ using

$$
\begin{aligned}
\widetilde{Y}\left(\widetilde{t}_{k-1}^{y}\right) & =\sum_{l=0}^{L} U\left(t_{l}^{u}\right)\left(G\left(\widetilde{t}_{k-1}^{y}-t_{l+1}^{u}\right)-G\left(-t_{l+1}^{u}\right)\right) \\
& -U\left(t_{l}^{u}\right)\left(G\left(\widetilde{t}_{k-1}^{y}-t_{l}^{u}\right)+G\left(-t_{l}^{u}\right)\right),
\end{aligned}
$$

where $G(t)=\int_{0}^{t} g(\tau) d \tau, U\left(t_{l}^{u}\right)=l \bar{\delta}-b t_{l}^{u}, l=0, \ldots, L$;

Step 2. Compute $\widetilde{t}_{k}^{y}$ using

$$
\widetilde{t}_{k}^{y}=\widetilde{f}_{k}\left(\widetilde{f}_{k}\left(\widetilde{t}_{k-1}^{y}\right)\right)=\frac{1}{b}\left(\bar{\delta}-\widetilde{Y}\left(\frac{1}{b}\left(\bar{\delta}-\widetilde{Y}\left(\widetilde{t}_{k-1}^{y}\right)\right)\right)\right)
$$

Using numerical simulations, Algorithm 6.1 is compared with the indirect method to show that the same accuracy can be achieved if the initial guess is chosen $t_{k, 0}^{y}=\widetilde{t}_{k-1}^{y}$, the previously computed spike time, and the recursive equation is computed twice (Algorithm step 2).

\subsection{Numerical study}

The simulations were carried out using the following second-order transfer function

$$
G(s)=\frac{1}{\tau^{2} s^{2}+s \zeta \tau s+1}
$$


where $\tau=10^{-2}, \zeta=1$. $G$ has impulse response function $g(t)=10^{4} \cdot t e^{-100 t}, t \geq 0$, which is plotted in Figure 6.2.

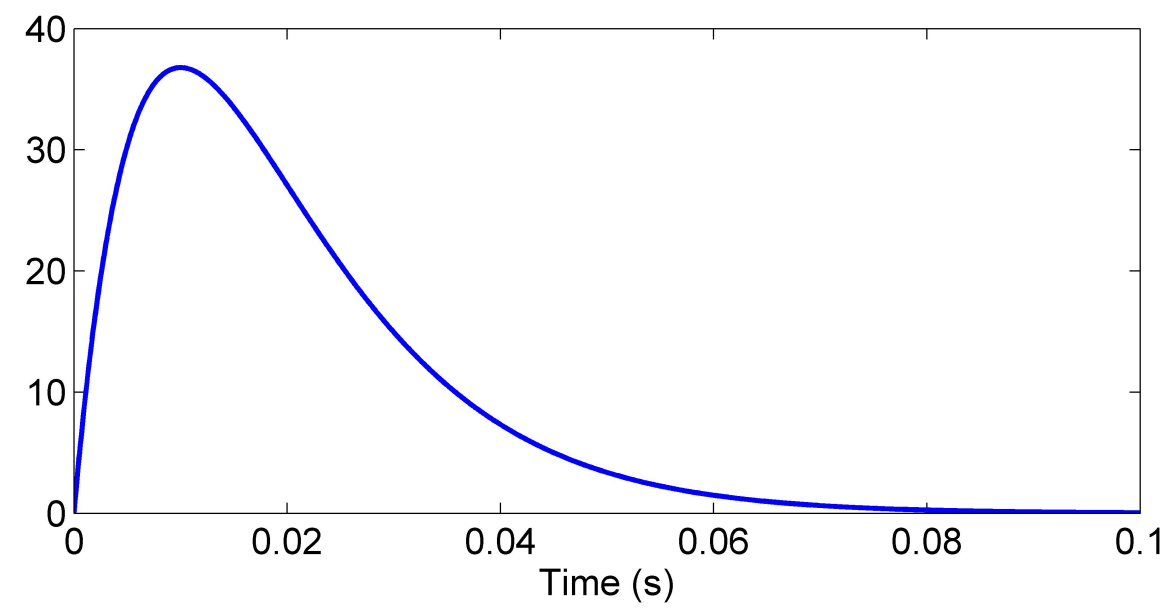

Figure 6.2: Impulse response of filter $G$.

\section{Example 1.}

In this example, simulations were carried out using the following bandlimited input

$$
u(t)=\sum_{k=1}^{15} c_{k} \cdot\left(\frac{\sin (\Omega(t-k \pi / \Omega))}{\Omega(t-k \pi / \Omega)}\right)^{2},
$$

where $c_{k}$ are random coefficients drawn from the uniform distribution on ] $-1,1$ [, and $\Omega=90$. The input was chosen to ensure that $u \in L^{1}(\mathbb{R})$. The bandwidth of $u$ is $2 \Omega=$ $180 \mathrm{rad} / \mathrm{s}$, due to the squared terms.

Filter $G$ responded with output $y$ when presented with input $u$, sampled with period $\varepsilon=2 \cdot 10^{-4}$. Functions $u$ and $y$, generated for $t \in[0,1]$, are depicted in Figure 6.3.

An ideal IF neuron with parameters $\bar{\delta}=10^{-2}, b=6$ was used to encode $u$ and $y$ into sequences $\left\{t_{l}^{u}\right\}_{l=0, \ldots, L}$ and $\left\{t_{k}^{y}\right\}_{k=0, \ldots, K}$, respectively, where $L=600$ and $K=599$. These parameters ensure that the reconstruction requirement (2.9) is satisfied.

The spike train $\left\{t_{l}^{u}\right\}_{l=0, \ldots, L}$ was used to compute predictions $\left\{\mathfrak{t}_{k}^{y_{1}}\right\}_{k=0, \ldots, K}$ using Algorithm 6.1.

Input $u$ was then reconstructed from sequence $\left\{t_{l}^{u}\right\}_{l=0, \ldots, L}$ with the standard algorithm for bandlimited functions (Lazar and Pnevmatikakis, 2008a). After simulating filter $G$ with this new reconstruction and encoding the output with the IF neuron, a new set of predictions $\left\{\tilde{t}_{k}^{y_{2}}\right\}_{k=0, \ldots, K}$ was computed.

The output spike time sequences calculated using the two algorithms were then used to 


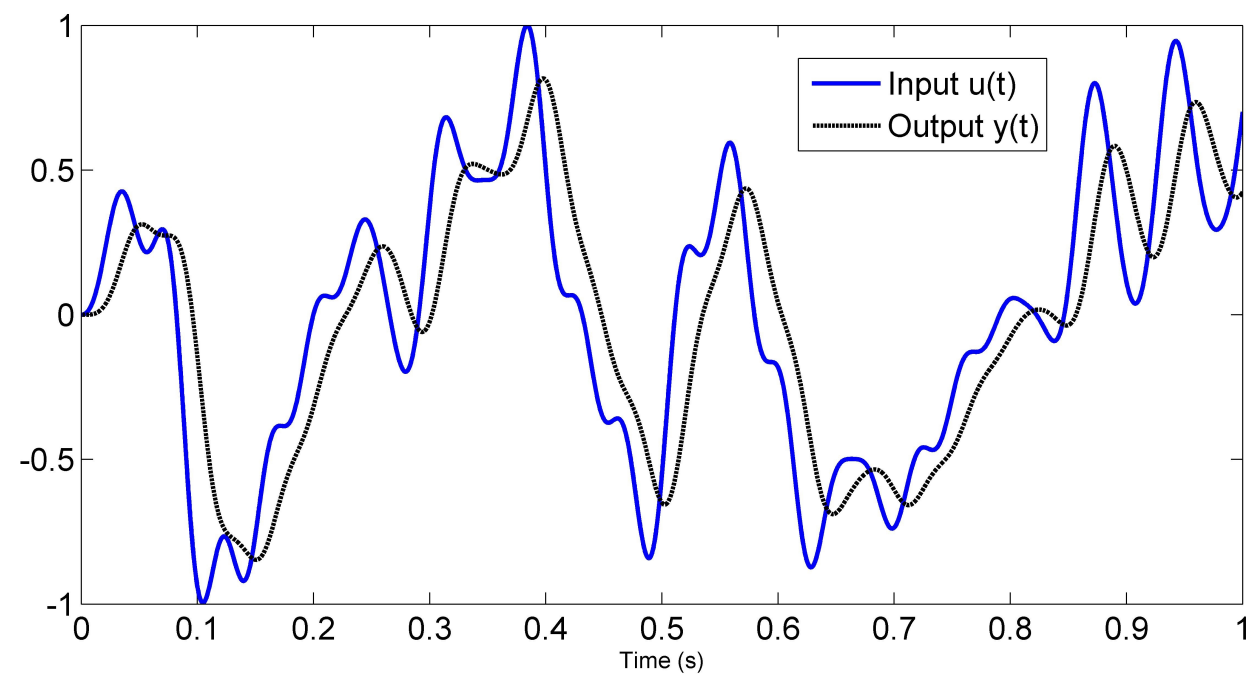

Figure 6.3: Input and output of filter $G$.

reconstruct back the output $y$ of the filter using the algorithm in (Lazar and Pnevmatikakis, 2008a). The following errors were evaluated

$$
\begin{aligned}
& e_{j}^{t}(k)=t_{k}^{y}-\widetilde{t}_{k}^{y_{j}}, \forall k=1, \ldots, K, \\
& e_{j}^{y}(t)=y(t)-\widetilde{y}_{j}(t), \forall t \in[0,1], j=1,2,
\end{aligned}
$$

where $e_{1}^{t}, e_{2}^{t}$ represent the timing errors introduced by direct and indirect methods and $e_{1}^{y}$, $e_{2}^{y}$ represent the errors between the reconstructed analog output signals and the true filter responses, introduced by direct and indirect methods, respectively.

To evaluate the accuracy of Algorithm 6.1, the mean-squared-errors (MSEs) were computed for the spike time sequences predicted by the direct and indirect approaches

$$
M S E_{j}=\frac{1}{K} \sum_{k=1}^{K}\left(e_{j}^{t}(k)\right)^{2}, j=1,2 .
$$

The resulted values for the MSEs are given in Table 6.1.

Table 6.1: Computing Times and MSEs of the Direct and Indirect Method for a Bandlimited Input

\begin{tabular}{|c||c||c|}
\hline Method & Computing Time & MSE \\
\hline Direct & $0.09 \mathrm{~s}$ & $3.57 \cdot 10^{-8}$ \\
\hline Indirect & $9.48 \mathrm{~s}$ & $1.89 \cdot 10^{-7}$ \\
\hline
\end{tabular}


The results show that the accuracy of the two methods is not significantly different. However, the computing time of the direct method is 2 orders of magnitude shorter than the one of the indirect approach.

The normalized errors for the reconstructed analog outputs for the direct and indirect methods $\left\{E_{j}\right\}_{j=1,2}$, where

$$
E_{j}(t)=\frac{\left|e_{j}^{y}(t)\right|}{\|y\|_{L^{2}}}, j=1,2
$$

are depicted in Figure 6.4.

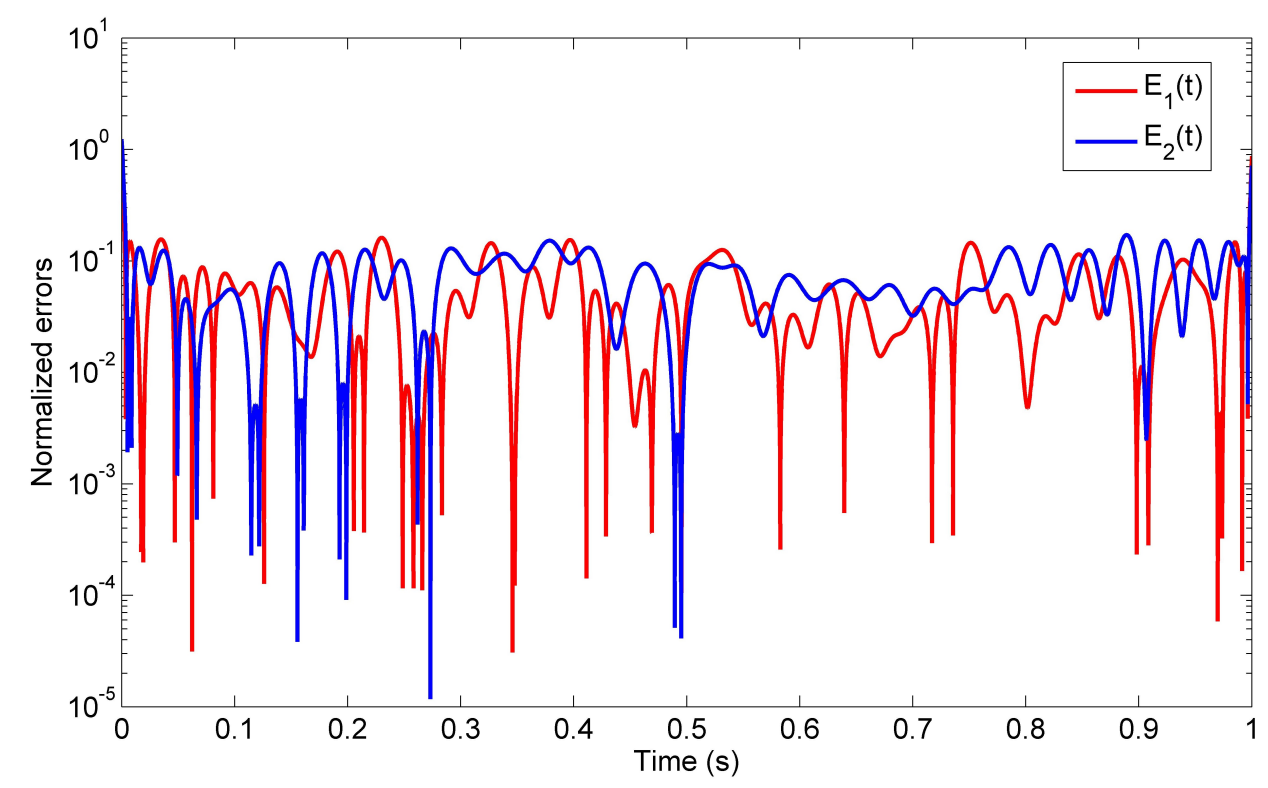

Figure 6.4: Normalized errors $E_{1}$ and $E_{2}$ for the direct and indirect methods, respectively.

\section{Example 2.}

The direct and indirect methods were further evaluated using a non-bandlimited input

$$
u(t)=\sum_{k=1}^{15} d_{k} \cdot \beta^{10}(t / T-k), t \in[0,1],
$$

where $d_{k}$ are random coefficients drawn from the uniform distribution on $]-1,1[, T=0.06$ and $\beta^{10}$ represents the $\mathrm{B}$-spline of degree 10 . Function $u$ was sampled with sampling time $\varepsilon=10^{-4}$. The input $u$ and the corresponding output $y$ of filter $G$ given in (6.14) are displayed in Figure 6.5.

An ideal IF neuron with parameters $\bar{\delta}=10^{-2}$ and $b=6$ was used to encode $u$ and $y$. The resulting spike train $\left\{t_{l}^{u}\right\}_{l=0, \ldots, L}$ was used to compute the output spike train 


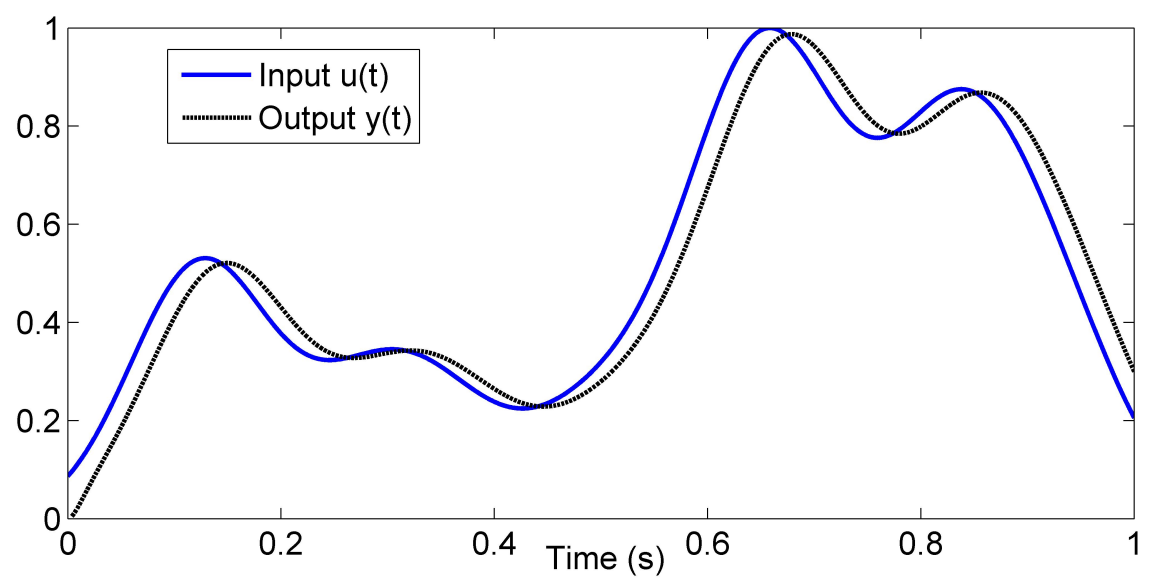

Figure 6.5: Input and output of filter G.

$\left\{\widetilde{t}_{k}^{y_{1}}\right\}_{k=0, \ldots, K}$ using Algorithm 6.1, where $L=653$ and $K=651$. The spike train $\left\{t_{l}^{u}\right\}_{l=0, \ldots, L}$ was reconstructed with the algorithm in (Lazar and Pnevmatikakis, 2009) and filter $G$ was simulated with this new reconstruction. The output was encoded with the same IF neuron to compute a new set of predictions $\left\{\widetilde{t}_{k}^{y_{2}}\right\}_{k=0, \ldots, K}$. The MSE errors were evaluated as in the previous example, and are displayed in Table 6.2.

Table 6.2: Computing Times and MSEs for the Direct and Indirect Method for a NonBandlimited Input

\begin{tabular}{|c||c||c|}
\hline Method & Computing Time & MSE \\
\hline Direct & $0.065 \mathrm{~s}$ & $1.092 \cdot 10^{-7}$ \\
\hline Indirect & $1.455 \mathrm{~s}$ & $1.991 \cdot 10^{-7}$ \\
\hline
\end{tabular}

The results show that the two methods have the same accuracy. However, the computing time of the direct method is 1 order of magnitude shorter than the one of the indirect approach.

\subsection{Conclusions}

This chapter introduces for the first time a direct relationship between the output of a linear filter encoded with an ideal IF neuron directly from the filter input encoded with the same IF neuron. This is used to derive a practical algorithm for computing the output spike time sequence directly from the time encoded input.

An advantage of the proposed algorithm is that it does not depend on the input space. In 
contrast, the reconstruction performed as part of the indirect method is implemented with an algorithm that is specific to the input space.

The proposed algorithm is compared through numerical simulations with the alternative indirect approach, which involves reconstructing the input, simulating the linear filter, and encoding the resulting output in time domain.

The two methods were tested for a bandlimited input function. The calculated MSE values were similar, while the computing time of the proposed algorithm was 2 orders of magnitude shorter than the one of the indirect method.

The methods were further tested for a non-bandlimited input generated using B-spline functions. While the algorithms had the same accuracies, the computing time of the proposed algorithm was significantly shorter than for the indirect method. 


\section{Chapter 7}

\section{Conclusions and future work}

In 2008 the National Academy of Engineering in the US has proposed a list of 14 grand challenges for engineering in the 21st century. One of the grand challenges identified in their report consists in the "reverse engineering" of the brain. However, in order to understand how the brain works, the development of new theoretical frameworks is required for inferring the general principles and underlying mechanisms of the brain function.

To this end, this thesis has proposed a new theoretical framework that reformulates the IF neuron encoding of analog signals into spike trains as a problem of uniform sampling on a set of input independent time points. New algorithms for reconstructing the IF input, belonging to bandlimited and shift-invariant spaces (SIS), were developed based on the new formulation. Through numerical simulations, it was demonstrated that the proposed algorithms are as accurate but significantly faster than the standard methods. Moreover, the rate of increase in computation time of the proposed algorithms, relative to the length of the processed spike sequence, is significantly lower than the rate of the standard methods.

In order to infer the algorithms that underpin the brain computation, the huge amount of neuroscience data available can be exploited for developing new methods and tools for modelling, analysing and simulating the neural circuits that make up the brain.

In this respect, the thesis has developed two new methodologies for identifying [Nonlinear Filter]-[Ideal IF] and [Linear Filter]-[Leaky IF] circuits consisting of two steps: the estimation of the spiking neuron parameters and the identification of the filter.

The methodologies are based on the reformulation of the circuit as a scaled filter in series with a modified spiking neuron. To evaluate the proposed approaches and demonstrate their applicability, numerical simulations studies were carried out in which [Nonlinear Filter]-[Ideal IF] circuits were identified directly from simulation data without making any assumptions on the structure and parameters of the nonlinear filter. Through numerical simulations, it was shown that the identified nonlinear filter was an accurate representation of the scaled original filter. 
Similarly, it was demonstrated that the proposed algorithm can identify an unknown [Linear Filter]-[Leaky IF] circuit by estimating first the leaky IF parameters with arbitrary precision using specific stimuli sequences. Subsequently, the structure and parameters of the linear filter were derived from the reconstructed filter output (the input to the leaky IF neuron) by applying the NARMAX system identification methodology.

An important challenge in neuromorphic engineering is the development of algorithms that perform mathematical computations in the spike domain.

In this respect, this thesis developed a new mathematical representation that relates directly the time encoded output and input of a linear filter, where the TEM is represented by an ideal IF neuron. A new practical algorithm was also proposed for computing the time encoded output directly from the input spike time sequence. A bound for the error between the real and estimated spike times is also derived, depending on the IF neuron parameters. Through numerical simulations, it was demonstrated that the proposed algorithm is significantly faster than the standard approach to computing the encoded filter output, which involves reconstructing the input from its corresponding spike sequence, simulating the linear filter, and subsequently encoding the resulting output into a spike train.

A better understanding of how the brain works will not only enable the treatment of brain disorders, the repair of damaged brains, or the improvement of machine learning techniques, but will also enable the development of microchips that mimic the neural architecture and implement similar information processing strategies.

The results presented in this thesis could be further developed is a number of ways.

- The new reconstruction algorithms introduced in Chapter 3 and Chapter 4 are developed exclusively for ideal IF neurons. It would be extremely useful to extend the framework to more complex neuron models like the leaky IF neuron, the IF neuron with random threshold or a population of IF neurons.

- Chapter 5 introduced a new identification methodology for [Linear Filter]-[Leaky IF] circuits. An improvement to this identification methodology can be made by extending it to [Nonlinear]-[Leaky IF] circuits. Moreover, different spiking neuron models can be considered.

- It would be useful to extend the representation of linear filters in the spike domain in Chapter 6 and the corresponding reconstruction algorithm to nonlinear filters. 


\section{Appendix A}

\section{An overview of Hilbert spaces and frames}

This appendix presents the generic definition of a linear space, and reviews the theory of normed linear spaces, Hilbert spaces and reproducing kernel Hilbert spaces (RKHSs). Further on, it defines the orthogonal basis and presents an introduction to the theory of frames.

More information on linear spaces, RKHSs or frames can be found in (Naylor and Sell, 1982), (Berlinet and Thomas-Agnan, 2004) or (Christensen, 2003), respectively.

Definition 7.1. The nonempty set $X$ is a linear space over scalar field $F$, together with a mapping of $X \times X$ into $X$ called addition, denoted $x_{1}+x_{2}$, and a mapping $F \times X$ into $X$ called scalar multiplication, denoted $\alpha x$, if the following conditions are satisfied

(A1) $x_{1}+x_{2}=x_{2}+x_{1}, \forall x_{1}, x_{2} \in X$;

(A2) $x_{1}+\left(x_{2}+x_{3}\right)=\left(x_{1}+x_{2}\right)+x_{3}, \forall x_{1}, x_{2}, x_{3} \in X$;

(A3) There exists a unique element $0 \in X$, such that $0+x=x, \forall x \in X$;

(A4) $\forall x \in X$, there exists a unique element $-x \in X$, such that $x+(-x)=0$;

(SM1) $\alpha_{1}\left(\alpha_{2} x\right)=\left(\alpha_{1} \alpha_{2}\right) x, \forall \alpha_{1}, \alpha_{2} \in F, \forall x \in X$;

(SM2) There exists a unique element $1 \in F$, such that $1 x=x, \forall x \in X$;

(SM3) There exists a unique element $0 \in F$, such that $0 x=0, \forall x \in X$;

(ASM1) $\alpha\left(x_{1}+x_{2}\right)=\alpha x_{1}+\alpha x_{2}, \forall \alpha \in F, \forall x_{1}, x_{2} \in X$

(ASM2) $\left(\alpha_{1}+\alpha_{2}\right) x=\alpha_{1} x+\alpha_{2} x, \forall \alpha_{1}, \alpha_{2} \in F, \forall x \in X$.

In order to define the convergence of a sequence in $X$, the linear space is required to satisfy additional properties. 
Definition 7.2. The function $\|\cdot\|: X \rightarrow \mathbb{R}_{+}$is called a norm on $X$ if

(N1) $\|x\| \geq 0, \forall x \in X$;

(N2) $\|x+y\| \leq\|x\|+\|y\|, \forall x, y \in X$;

(N3) $\|\alpha x\|=|\alpha| \cdot\|x\|, \forall \alpha \in F, \forall x \in X$;

(N4) $\|x\|=0 \Leftrightarrow x=0$.

Definition 7.3. The pair $(X,\|\cdot\|)$ is called a normed linear space if $X$ is a linear space and $\|\cdot\|$ is a norm defined on $X$.

Definition 7.4. The sequence $\left\{x_{n}\right\}_{n \in \mathbb{N}}$ is called a Cauchy sequence in normed linear space $(X,\|\cdot\|)$ if $\forall \varepsilon>0, \exists N_{\varepsilon}>0$ such that $\left\|x_{n}-x_{m}\right\|<\varepsilon, \forall n, m>N_{\varepsilon}$.

Definition 7.5. The sequence $\left\{x_{n}\right\}_{n \in \mathbb{N}}$ is convergent in normed linear space $(X,\|\cdot\|)$ if $\exists x_{0} \in(X,\|\cdot\|)$ satisfying $\forall \varepsilon>0, \exists N_{\varepsilon}>0$ such that $\left\|x_{n}-x_{0}\right\|<\varepsilon, \forall n>N_{\varepsilon}$.

Any sequence $\left\{x_{n}\right\}_{n \in \mathbb{N}}$ that is convergent in $(X,\|\cdot\|)$ is also a Cauchy sequence in $(X,\|\cdot\|)$, but the converse is not always true (Naylor and Sell, 1982).

Definition 7.6. A normed linear space $(X,\|\cdot\|)$ is called complete if each Cauchy sequence in $(X,\|\cdot\|)$ is convergent in $(X,\|\cdot\|)$.

Definition 7.7. An inner product on complex linear space $X$ is a mapping $\langle\cdot, \cdot\rangle: X \times X \rightarrow$ $\mathbb{C}$ that satisfies

$$
\begin{aligned}
& \text { (IP1) }\langle x+y, z\rangle=\langle x, z\rangle+\langle y, z\rangle, \forall x, y, z \in X ; \\
& \text { (IP2) }\langle\alpha x, y\rangle=\alpha\langle x, y\rangle, \forall x, y \in X, \forall \alpha \in \mathbb{C} ; \\
& \text { (IP3) }\langle x, y\rangle=\langle y, x\rangle^{*}, \forall x, y \in X ; \\
& \text { (IP4) }\langle x, x\rangle>0, \forall x \in X, x \neq 0 .
\end{aligned}
$$

Definition 7.8. A Hilbert space is a complete normed linear space $(\mathcal{H},\|\cdot\|)$ together with an inner product defined on $\mathcal{H}$. The norm and the inner product will be denoted by $\|\cdot\|_{\mathcal{H}}$ and $\langle\cdot, \cdot\rangle_{\mathcal{H}}$, respectively. When no confusion is likely, $(\mathcal{H},\|\cdot\|)$ will be denoted by $\mathcal{H}$.

Example 1. A well known Hilbert space is $L^{2}(\mathbb{R})$, the space of real functions of finite energy, defined by

$$
L^{2}(\mathbb{R}) \triangleq\left\{f: \mathbb{R} \rightarrow \mathbb{R}: \int_{-\infty}^{\infty}|f(x)|^{2} d x<\infty\right\}
$$


The inner product on $L^{2}(\mathbb{R})$ is defined by

$$
\langle f, g\rangle_{L^{2}} \triangleq \int_{-\infty}^{\infty} f(x) g(x) d x, \forall f, g \in L^{2}(\mathbb{R}),
$$

and the norm has the expression $\|f\|_{L^{2}} \triangleq \sqrt{\langle f, f\rangle_{L^{2}}}$.

Example 2. The Hilbert space $l^{2}(\mathbb{R})$ is defined by

$$
l^{2}(\mathbb{R}) \triangleq\left\{c=\left\{c_{k}\right\}_{k \in \mathbb{Z}}, c_{k} \in \mathbb{R}, \forall k \in \mathbb{Z}: \sum_{k \in \mathbb{Z}}\left|c_{k}\right|^{2}<\infty\right\}
$$

with inner product $\langle c, d\rangle_{l^{2}} \triangleq \sum_{k \in \mathbb{Z}} c_{k} d_{k}$ and norm $\|c\|_{l^{2}}=\sqrt{\langle c, c\rangle_{l^{2}}}, \forall c, d \in l^{2}(\mathbb{R})$.

Definition 7.9. Let $\mathcal{H}$ be a Hilbert space. Operator $\mathcal{C}: \mathcal{H} \rightarrow \mathbb{C}$ is called bounded if $\exists M>0$ such that $|\mathcal{C} f|<M\|f\|_{\mathcal{H}}, \forall f \in \mathcal{H}$.

Definition 7.10. A RKHS is a Hilbert space $\mathcal{H}$ of functions $f$ on domain $D$ such that the linear operator

$$
\mathcal{K}_{x}: \mathcal{H} \rightarrow \mathbb{R}, \mathcal{K}_{x} f=f(x)
$$

is bounded $\forall x \in D$.

Definition 7.11. Let $\mathcal{H}$ be a RKHS of functions on domain $D$. Then the unique function $K: D \times D \rightarrow \mathbb{R}$ that satisfies $\mathcal{K}_{x} f=\langle K(x, \cdot), f\rangle_{\mathcal{H}}, \forall x \in D$, is called the reproducing kernel of $\mathcal{H}$.

Example 3. The Paley-Wiener space of bandwidth $\Omega$ is defined by

$$
P W_{\Omega}=\left\{u \in L^{2}(\mathbb{R}): \operatorname{supp}(\widehat{u}) \subseteq[-\Omega, \Omega]\right\},
$$

where $\widehat{u}$ denotes the Fourier transform of function $u$ and $\operatorname{supp}(\widehat{u})$ denotes the support of $\widehat{u}$. Then $P W_{\Omega}$ is a Hilbert space with norm $\|\cdot\|_{L^{2}}$ and inner product $\langle\cdot, \cdot\rangle_{L^{2}}$. Furthermore, it is a RKHS with reproducing kernel (Berlinet and Thomas-Agnan, 2004)

$$
K(\cdot, x)=g_{\Omega}(\cdot-x) \triangleq \frac{\sin (\Omega(t-x))}{\pi(t-x)} .
$$

Therefore, the uniform samples of a function $f \in P W_{\Omega}$ satisfy

$$
f(k T)=\langle f, g(\cdot-k T)\rangle_{L^{2}}, \forall k \in \mathbb{Z}
$$

Example 4. The space of trigonometric polynomials of bandwidth $\Omega$ is defined by

$$
\mathcal{H}_{\Omega}^{M}=\left\{u: \mathbb{R} \rightarrow \mathbb{C}: u(t)=\sum_{m=-M}^{M} a_{m} e^{j m \frac{\Omega}{M} t}\right\} .
$$


Functions $u \in \mathcal{H}_{\Omega}^{M}$ are periodic of period $T=\frac{2 \pi M}{\Omega}$. Then $\mathcal{H}_{\Omega}^{M}$ is a Hilbert space with inner product

$$
\langle u, v\rangle_{\mathcal{H}_{\Omega}^{M}} \triangleq \int_{-T / 2}^{T / 2} u(\tau) \overline{v(\tau)} d \tau
$$

The norm in $\mathcal{H}_{\Omega}^{M}$ is defined by $\|u\|_{\mathcal{H}_{\Omega}^{M}} \triangleq \sqrt{\langle u, u\rangle_{\mathcal{H}_{\Omega}^{M}}}$. Furthermore, $\mathcal{H}_{\Omega}^{M}$ is a RKHS with reproducing kernel (Lazar et al., 2010)

$$
K_{M}(\cdot, x)=\frac{2 M+1}{T} \frac{\operatorname{sinc}\left(\frac{(2 M+1) \Omega}{2 M}(\cdot-x)\right)}{\operatorname{sinc}\left(\frac{\Omega}{2 M}(\cdot-x)\right)},
$$

where $\operatorname{sinc}(t) \triangleq \frac{\sin (t)}{t}, \forall t \in \mathbb{R}$.

Definition 7.12. A set of elements $\left\{f_{k}\right\}_{k \in \mathbb{Z}}$ in Hilbert space $\mathcal{H}$ is called orthonormal if

$$
\left\langle f_{n}, f_{k}\right\rangle_{\mathcal{H}}=\delta_{n k}, \forall n, k \in \mathbb{Z},
$$

where $\delta_{n k}$ is the Kronecker function.

Definition 7.13. An orthonormal set of elements $S=\left\{f_{k}\right\}_{k \in \mathbb{Z}}$ in Hilbert space $\mathcal{H}$ is called maximal if $\nexists g \in \mathcal{H} \backslash S$ such that $S \cup\{g\}$ is orthonormal.

Definition 7.14. A maximal orthonormal set $S$ in a Hilbert space $\mathcal{H}$ is called an orthonormal basis for $\mathcal{H}$.

A property of an orthonormal basis $S=\left\{f_{k}\right\}_{k \in \mathbb{Z}}$ in $\mathcal{H}$, also known as the Fourier series expansion, is that any function $f \in \mathcal{H}$ satisfies

$$
f=\sum_{k \in \mathbb{Z}}\left\langle f, f_{k}\right\rangle_{\mathcal{H}} \cdot f_{k}
$$

Example 5. The set of functions $S=\left\{f_{k}\right\}_{k \in \mathbb{Z}}$ where $f_{k}=\sqrt{\frac{\Omega}{\pi}} \cdot \operatorname{sinc}(\Omega(\cdot-k T)), \forall k \in$ $\mathbb{Z}, T \triangleq \pi / \Omega$ forms an orthonormal basis in $P W_{\Omega}$ (Naylor and Sell, 1982). Furthermore, the coefficients $\left\{c_{k}\right\}_{k \in \mathbb{Z}}$ of a function $f \in P W_{\Omega}$ satisfy

$$
c_{k}=\left\langle f, f_{k}\right\rangle_{L^{2}}=\sqrt{\frac{\pi}{\Omega}} \cdot f(k T), \forall k \in \mathbb{Z} .
$$

Example 6. The set of functions $\left\{\frac{1}{\sqrt{T}} e^{j m \frac{\Omega}{M} t}\right\}_{m=-M, \ldots, M}$ forms an orthonormal basis on $\mathcal{H}_{\Omega}^{M}$, where $T=\frac{2 \pi M}{\Omega}$. Moreover, $\lim _{M \rightarrow \infty} \mathcal{H}_{\Omega}^{M}=P W_{\Omega}$ (Lazar et al., 2010).

The basis in a Hilbert space consists of a set of linearly independent elements that spans the whole space. The more generic concept of frame, introduced by Duffin and Schaeffer (1952), consists of elements that are not always linearly independent, and can thus be used for a wider range of applications. 
Definition 7.15. A sequence $\left\{f_{k}\right\}_{k \in \mathbb{Z}}$ in a Hilbert space $\mathcal{H}$ is called a frame with bounds $A, B$ if

$$
A\|f\|_{\mathcal{H}}^{2} \leq \sum_{k \in \mathbb{Z}}\left|\left\langle f, f_{k}\right\rangle_{\mathcal{H}}\right|^{2} \leq B\|f\|_{\mathcal{H}}^{2}, \forall f \in \mathcal{H}
$$

Frame $\left\{f_{k}\right\}_{k \in \mathbb{Z}}$ is an orthonormal basis if and only if $A=B=1$. A property weaker than being a frame, for a sequence $\left\{f_{k}\right\}_{k \in \mathbb{Z}}$, is defined as follows.

Definition 7.16. A sequence $\left\{f_{k}\right\}_{k \in \mathbb{Z}}$ is a Bessel sequence with bound B for Hilbert space $\mathcal{H}$ if

$$
\sum_{k \in \mathbb{Z}}\left|\left\langle f, f_{k}\right\rangle\right|^{2} \leq B\|f\|_{\mathcal{H}}^{2}, \forall f \in \mathcal{H}
$$

Definition 7.17. Let $\mathcal{H}_{1}$ and $\mathcal{H}_{2}$ be two Hilbert spaces. The adjoint of linear operator $\mathcal{O}: \mathcal{H}_{1} \rightarrow \mathcal{H}_{2}$ is the unique operator $\mathcal{O}^{*}: \mathcal{H}_{2} \rightarrow \mathcal{H}_{1}$ that satisfies

$$
\langle\mathcal{O} f, g\rangle_{\mathcal{H}_{2}}=\left\langle f, \mathcal{O}^{*} g\right\rangle_{\mathcal{H}_{1}}, \forall f \in \mathcal{H}_{1}, \forall g \in \mathcal{H}_{2}
$$

Operator $\mathcal{O}$ is called self-adjoint if $\mathcal{H}_{1}=\mathcal{H}_{2}$ and $\mathcal{O}^{*}=\mathcal{O}$.

Every frame is associated with two operators, defined as follows.

Definition 7.18. For a frame $\left\{f_{k}\right\}_{k \in \mathbb{Z}}$ in Hilbert space $\mathcal{H}$, the operator

$$
\mathcal{C}: l^{2}(\mathbb{R}) \rightarrow \mathcal{H}, \mathcal{C} d=\sum_{k \in \mathbb{Z}} d_{k} f_{k}, \forall d \in l^{2}(\mathbb{R})
$$

is called the synthesis operator. Its adjoint,

$$
\mathcal{C}^{*}: \mathcal{H} \rightarrow l^{2}(\mathbb{R}),\left[\mathcal{C}^{*} f\right]_{k}=\left\langle f, f_{k}\right\rangle_{\mathcal{H}}, \forall k \in \mathbb{Z}, \forall f \in \mathcal{H},
$$

is called the analysis operator. Moreover, the space $\mathcal{H}$ is called the function space, while $l^{2}(\mathbb{R})$ is called the sequence space.

Inequalities (7.2) are equivalent to

$$
A\|f\|_{\mathcal{H}}^{2} \leq\left\|\mathcal{C}^{*} f\right\|_{l^{2}}^{2} \leq B\|f\|_{\mathcal{H}}^{2}, \forall f \in \mathcal{H}
$$

The first inequality in (7.3) is a sufficient condition for $\mathcal{C}^{*}$ to be one-to-one. The second inequality proves that $\mathcal{C}^{*}$ is bounded, and thus continuous (Naylor and Sell, 1982). Therefore, $f$ is uniquely and continuously associated with $\left\{\left\langle f, f_{k}\right\rangle_{\mathcal{H}}\right\}_{k \in \mathbb{Z}}$, also known as the measurements of $f$ (Eldar and Werther, 2005, Eldar, 2003).

For a frame $\left\{f_{k}\right\}_{k \in \mathbb{Z}}$, the operator $\mathcal{S}: \mathcal{H} \rightarrow \mathcal{H}, \mathcal{S} f \triangleq \mathcal{C C}^{*} f=\sum_{k=-\infty}^{\infty}\left\langle f, f_{k}\right\rangle_{\mathcal{H}} \cdot f_{k}$ is called the frame operator. Operator $\mathcal{S}$ is invertible, bounded and self-adjoint (Christensen, 
2003). Therefore, a function $f \in P W_{\Omega}$ can be expanded as

$$
\begin{aligned}
f=\mathcal{S}^{-1} \mathcal{S} f & =\sum_{k=-\infty}^{\infty}\left\langle f, \tilde{f}_{k}\right\rangle_{\mathcal{H}} \cdot f_{k} \\
& =\sum_{k=-\infty}^{\infty}\left\langle f, f_{k}\right\rangle_{\mathcal{H}} \cdot \tilde{f}_{k}
\end{aligned}
$$

where $\tilde{f}_{k} \triangleq \mathcal{S}^{-1} f_{k}, \forall k \in \mathbb{Z}$. Moreover, $\left\{\tilde{f}_{k}\right\}_{k \in \mathbb{Z}}$ is a frame with lower and upper bounds $B^{-1}, A^{-1}$, respectively, called the dual frame (Christensen, 2003)

$$
B^{-1}\|f\|_{\mathcal{H}}^{2} \leq \sum_{k \in \mathbb{Z}}\left|\left\langle f, \tilde{f}_{k}\right\rangle_{\mathcal{H}}\right|^{2} \leq A^{-1}\|f\|_{\mathcal{H}}^{2}, \forall f \in \mathcal{H}
$$

The expressions in (7.4) and (7.5) are called frame expansion and dual frame expansion, respectively (Benedetto, 1992). If $\left\{f_{k}\right\}_{k \in \mathbb{Z}}$ is an orthonormal basis on $P W_{\Omega}$, the expressions (7.4) and (7.5) are both equal to the Fourier series expansion (7.1). 


\section{Appendix B \\ Proof of theorems}

This appendix contains the proofs of Theorem 5.2 and Corollary 4.2. In order to prove Theorem 5.2 the following auxiliary lemma is necessary.

Lemma 7.1. Let $\left.\Lambda_{y}:\right] 1,+\infty[\rightarrow] 1,+\infty\left[, \Lambda_{y}(s)=\frac{1}{1-\left(1-\frac{1}{s}\right)^{y}}, y \in\right] 0,+\infty[$.

Then $\Lambda_{y}$ is strictly concave for $y<1$, and strictly convex for $y>1$.

Proof. The following holds true.

$$
\Lambda_{y}^{\prime}(s)=\frac{y}{s^{2}\left(\left(\frac{s}{s-1}\right)^{\frac{y-1}{2}}-\left(\frac{s-1}{s}\right)^{\frac{y+1}{2}}\right)^{2}}=y \frac{((s-1) s)^{y-1}}{\left(s^{y}-(s-1)^{y}\right)^{2}}
$$

and

$$
\Lambda_{y}^{\prime \prime}(s)=\frac{((s-1) s)^{y-2} h(s)}{\left(s^{y}-(s-1)^{y}\right)^{3}}
$$

where

$$
h(s)=(y-1)(2 s-1)\left(s^{y}-(s-1)^{y}\right)-2 y s(s-1)\left(s^{y-1}-(s-1)^{y-1}\right) .
$$

It can be observed that $\left.\operatorname{sgn}\left(\Lambda_{y}^{\prime \prime}(s)\right)=\operatorname{sgn}(p(s)), \forall s \in\right] 1,+\infty[$. After simple calculations, it follows that

$$
\begin{aligned}
h(s) & =(s-1)^{y}(2 s+y-1)-s^{y}(2 s-y-1) \\
& =s^{y}(2 s+y-1)\left(\left(\frac{s-1}{s}\right)^{y}-\frac{2 s-y-1}{2 s+y-1}\right) .
\end{aligned}
$$

In order to assess the sign of $h$, the following function is calculated

$$
h(\lambda(p))=\left(\frac{1}{1-p}\right)^{y}\left(\frac{2}{1-p}+y-1\right)\left(p^{y}-\frac{p(1+y)+(1-y)}{p(1-y)+(1+y)}\right),
$$


where $\lambda:] 0,1[\rightarrow] 1,+\infty\left[, \lambda(p) \triangleq \frac{1}{1-p}, \forall p \in\right] 0,1[$. The following holds

$$
\left.\left(\frac{1}{1-p}\right)^{y}\left(\frac{2}{1-p}+y-1\right)>0, \forall p \in\right] 0,1[
$$

Case I. $y<1$.

In this case $p(1+y)+(1-y)>0, \forall p, y \in] 0,1[$. It follows that $\operatorname{sgn}(h(\lambda(p)))=$ $\operatorname{sgn}(\theta(p)), \forall p \in] 0,1[$, where $\theta:] 0,1[\rightarrow \mathbb{R}$,

$$
\theta(p) \triangleq y \cdot \ln (p)-\ln \left(\frac{p(1+y)+(1-y)}{p(1-y)+(1+y)}\right),
$$

such that $p(1-y)+(1+y)>0, \forall p \in] 0,1[$. Furthermore,

$$
\begin{aligned}
\theta^{\prime}(p) & =\frac{y}{p}-\frac{4 y}{(p(1-y)+(1+y))(p(1+y)+(1-y))} \\
& =\frac{y\left(1-y^{2}\right)(p-1)^{2}}{p(p(1-y)+(1+y))(p(1+y)+(1-y))} .
\end{aligned}
$$

Then $\left.\theta^{\prime}(p)>0, \forall p \in\right] 0,1\left[\right.$ and $\lim _{p \rightarrow 1} \theta(p)=0$. It follows that $\theta(p)<0, h(\lambda(p))<$ $0, \forall p \in] 0,1\left[, h(s)<0, \Lambda_{y}^{\prime \prime}(s)<0, \forall s \in\right] 1,+\infty[$, and thus the lemma holds true.

Case II. $y>1$. The following holds.

$$
\begin{aligned}
& \left.p(1+y)+(1-y) \leq 0, p \in] 0, p_{0}\right] \\
& p(1+y)+(1-y)>0, p \in] p_{0}, 1[
\end{aligned}
$$

where $p_{0}=\frac{y-1}{y+1}$.

For $\left.p \in] 0, p_{0}\right]$ it follows that $\left.\left.h(\lambda(p))>0, \forall p \in\right] 0, p_{0}\right]$ (7.6), and thus $h(s)>$ $\left.0, \Lambda_{y}^{\prime \prime}(s)>0, \forall s \in\right] 1, \frac{1}{1-p_{0}}[$.

For $p \in] p_{0}, 1\left[, \theta\right.$ and $\theta^{\prime}$ are calculated as in Case I. Then $\theta^{\prime}(p)<0, \lim _{p \rightarrow 1} \theta(p)=0$, $\theta(p)>0, \forall p \in] p_{0}, 1[$, and the result follows.

Proof of Theorem 5.2. The values $\left\{I S I_{n}\right\}_{n=0,1,2}$ satisfy (5.19)

$$
I S I_{n}=-R C \cdot \ln \left(a_{n}\right), \forall n=0,1,2
$$

where $a_{n} \triangleq 1-\frac{\bar{\delta}_{b}}{R C\left(K_{b} u_{n}+1\right)}, n=0,1,2$, and

$$
\left.u_{n} \in\right]-\frac{R C-\bar{\delta}_{b}}{K_{b} \cdot R C}, \frac{R C-\bar{\delta}_{b}}{K_{b} \cdot R C}[, n=0,1,2 .
$$

It follows that $0<a_{2}<a_{0}<a_{1}<1$. Moreover, 


$$
f(x)=\frac{1-a_{1}^{\frac{R C}{x}}}{1-2 a_{1}^{\frac{R C}{x}}+a_{0}^{\frac{R C}{x}}}-\frac{1-a_{2}^{\frac{R C}{x}}}{1-a_{0}^{\frac{R C}{x}}} .
$$

It can be easily verified that $x=R C$ is a solution to $f(x)=0$, by substituting the expressions of $\left\{a_{n}\right\}_{n=0,1,2}$ in (7.9).

Let $y>0, y \triangleq \frac{R C}{x}$. Then the following holds.

$$
\begin{aligned}
f(x)=0 & \Leftrightarrow \frac{1-2 a_{1}^{y}+a_{0}^{y}}{1-a_{1}^{y}}=\frac{1-a_{0}^{y}}{1-a_{2}^{y}} \\
& \Leftrightarrow \frac{\left(2-2 a_{1}^{y}\right)-\left(1-a_{0}^{y}\right)}{1-a_{1}^{y}}=\frac{1-a_{0}^{y}}{1-a_{2}^{y}} \\
& \Leftrightarrow \frac{2}{1-a_{0}^{y}}=\frac{1}{1-a_{1}^{y}}-\frac{1}{1-a_{2}^{y}} .
\end{aligned}
$$

Let $\lambda:] 0,1[\rightarrow] 1,+\infty[$ be a strictly increasing and continuous function with expression $\left.\lambda(p) \triangleq \frac{1}{1-p}, \forall p \in\right] 0,1[$. Then $\lambda$ is a one-to-one and onto function, and thus it has an inverse $\left.\lambda^{-1}:\right] 1,+\infty[\rightarrow] 0,1\left[, \lambda^{-1}(s)=1-\frac{1}{s}\right.$. Equation (7.10) is satisfied for $y=1$, such that

$$
2 \lambda\left(a_{0}\right)=\lambda\left(a_{1}\right)+\lambda\left(a_{2}\right) .
$$

Let $s_{n}=\lambda\left(a_{n}\right), n=0,1,2$, and let $\left.\Lambda_{y}:\right] 1,+\infty[\rightarrow] 1,+\infty\left[, \Lambda_{y}(s)=\lambda\left(\lambda^{-1}(s)^{y}\right)\right.$. Then the following holds true (7.10)

$$
\begin{aligned}
2 s_{0} & =s_{1}+s_{2} \\
2 \Lambda_{y}\left(s_{0}\right) & =\Lambda_{y}\left(s_{1}\right)+\Lambda_{y}\left(s_{2}\right) .
\end{aligned}
$$

Function $\Lambda_{y}$ is strictly convex for $y>1$, due to Lemma 7.1, and thus

$$
2 \Lambda_{y}\left(s_{0}\right)<\Lambda_{y}\left(s_{1}\right)+\Lambda_{y}\left(s_{2}\right), y>1
$$

Similarly, Lemma 7.1 proves that $\Lambda_{y}$ is strictly concave for $y<1$, and thus

$$
2 \Lambda_{y}\left(s_{0}\right)>\Lambda_{y}\left(s_{1}\right)+\Lambda_{y}\left(s_{2}\right), y<1
$$

Therefore there is a unique solution $y \in] 0,+\infty[$ to equation (7.10). Moreover, the fol- 
lowing holds, which concludes the proof

$$
\begin{aligned}
\operatorname{sgn}(f(x)) & =-\operatorname{sgn}\left(\frac{1-2 a_{1}^{\frac{R C}{x}}+a_{0}^{y}}{1-a_{1}^{\frac{R C}{x}}}-\frac{1-a_{0}^{\frac{R C}{x}}}{1-a_{2}^{\frac{R C}{x}}}\right) \\
& =-\operatorname{sgn}\left(2 \Lambda_{\frac{R C}{x}}\left(s_{0}\right)-\Lambda_{\frac{R C}{x}}\left(s_{1}\right)+\Lambda_{\frac{R C}{x}}\left(s_{2}\right)\right) \\
& =-\operatorname{sgn}(x-R C), \forall x \in] 0,+\infty[
\end{aligned}
$$

Proof of Corollary 4.2. The Fourier coefficients of $\left(G_{\lambda}^{T}\right)^{2}$ have the expression

$$
\begin{aligned}
c_{-k} & =\frac{1}{2 \pi} \int_{0}^{2 \pi} \sum_{l \in \mathbb{Z}}\left|\widehat{\lambda}\left(\frac{\omega+2 l \pi}{T}\right)\right|^{2} e^{j k \omega} d \omega=\frac{1}{2 \pi} \int_{\mathbb{R}}\left|\widehat{\lambda}\left(\frac{\omega}{T}\right)\right|^{2} e^{j k \omega} d \omega \\
& =\frac{T}{2 \pi} \int_{\mathbb{R}}|\widehat{\lambda}(\omega)|^{2} e^{j k T \omega} d \omega=T\left\langle\widehat{\lambda}, \widehat{\lambda} \cdot e^{-j k T}\right\rangle_{L^{2}}=T\langle\lambda, \lambda(\cdot-k T)\rangle_{L^{2}}, \forall k \in \mathbb{Z} .
\end{aligned}
$$

Similarly,

$$
c_{-k}^{\prime}=T\left\langle\lambda^{\prime}, \lambda^{\prime}(\cdot-k T)\right\rangle_{L^{2}}, \forall k \in \mathbb{Z} .
$$

Therefore the result follows for a function $\lambda$ that satisfies $\operatorname{supp}(\lambda)=[-S T, S T]$.

The following holds

$$
K_{T}(x, t)=\frac{1}{T} K\left(\frac{x}{T}, \frac{t}{T}\right),
$$

where $K$ denotes the reproducing kernel of $V^{2}\left(\lambda_{T}\right), \lambda_{T}=\lambda(T \cdot)$, which satisfies (Gontier and Vetterli, 2014)

$$
K(x, t)=\sum_{j \in \mathbb{Z}} \tilde{\lambda}_{T}(x-j) \lambda_{T}(t-j)
$$

where $\left\{\tilde{\lambda}_{T}(t-j)\right\}_{j \in \mathbb{Z}}$ represents the bi-orthogonal frame of frame $\left\{\lambda_{T}(t-j)\right\}_{j \in \mathbb{Z}}$ satisfying (Gontier and Vetterli, 2014)

$$
\widehat{\vec{\lambda}}_{T}(\omega)=\frac{\widehat{\lambda}_{T}(\omega)}{\left(G_{\lambda_{T}}(\omega)\right)^{2}} .
$$

The following was proven in Theorem 4.2

$$
G_{\lambda_{T}}(\omega)=\frac{1}{T} G_{\lambda}^{T}(\omega), \forall \omega \in[0,2 \pi[
$$


Function $\widetilde{\lambda}_{T}$ is calculated as follows.

$$
\tilde{\lambda}_{T}=T^{2} \mathcal{F}^{-1}\left\{\widehat{\lambda}_{T}(\omega) \sum_{k \in \mathbb{Z}} d_{-k} e^{-i k \omega}\right\}=T^{2} \sum_{k \in \mathbb{Z}} d_{-k} \lambda_{T}(\cdot-k),
$$

where $\left\{d_{k}\right\}_{k \in \mathbb{Z}}$ are the Fourier coefficients of $\left(G_{\lambda}^{T}(\omega)\right)^{-2}$ and $\mathcal{F}^{-1}$ denotes the inverse Fourier transform operator. The following holds from (7.13)

$$
K_{T}(x, t)=T \sum_{j \in \mathbb{Z}}\left[\sum_{k \in \mathbb{Z}} d_{-k} \lambda(x-(j+k) T)\right] \lambda(t-j T) .
$$

The corollary holds true from (7.14) and $\operatorname{supp}(\lambda(\cdot-j T))=[(j-S) T,(j+S) T]$. 


\section{Bibliography}

H. Akaike. Fitting autoregressive models for prediction. Annals of the institute of Statistical Mathematics, 21(1):243-247, 1969.

H. Akaike. Statistical predictor identification. Annals of the Institute of Statistical Mathematics, 22(1):203-217, 1970.

A. Aldroubi and H. Feichtinger. Exact iterative reconstruction algorithm for multivariate irregularly sampled functions in spline-like spaces: The $l^{p}$-theory. Proceedings of the American Mathematical Society, 126(9):2677-2686, 1998.

A. Aldroubi and K. Gröchenig. Nonuniform sampling and reconstruction in shift-invariant spaces. SIAM review, 43(4):585-620, 2001.

A. Aldroubi, M. Unser, and A. Aldroubi. Sampling procedures in function spaces and asymptotic equivalence with shannon's sampling theory. Numerical functional analysis and optimization, 15(1-2):1-21, 1994.

P. Bartolini, E.C. Carcano, L. Piroddi, and J.B. Valdes. Forecasting daily streamflows using narmax models: How disturbances may affect model performance. In World Environmental and Water Resources Congress 2008, pages 1-13. ASCE, 2008.

R. Bashirullah, J.G. Harris, J.C. Sanchez, T. Nishida, and J.C. Principe. Florida wireless implantable recording electrodes (fwire) for brain machine interfaces. In Circuits and Systems, 2007. ISCAS 2007. IEEE International Symposium on, pages 2084-2087. IEEE, 2007.

J.J. Benedetto. Wavelets: A Tutorial in Theory and Applications, chapter Irregular sampling and frames, pages 445-507. Academic Press, Boston, 1992.

S. Bergner, T. Möller, D. Weiskopf, and D.J. Muraki. A spectral analysis of function composition and its implications for sampling in direct volume visualization. Visualization and Computer Graphics, IEEE Transactions on, 12(5):1353-1360, 2006. 
A. Berlinet and C. Thomas-Agnan. Reproducing kernel Hilbert spaces in probability and statistics, volume 3. Kluwer Academic Boston, 2004.

A. Beurling and P. Malliavin. On the closure of characters and the zeros of entire functions. Acta Mathematica, 118(1):79-93, 1967.

S. Billings and S. Chen. Extended model set, global data and threshold model identification of severely non-linear systems. International Journal of Control, 50(5):1897-1923, 1989.

S. Billings and M. Fadzil. The practical identification of systems with nonlinearities. Technical report, Dept of Automatic Control and System Engineering. University of Sheffield, 1984.

S. Billings and I. Leontaritis. Parameter estimation techniques for nonlinear systems. In 6 th IFAC Symp. Identification and System Parameter Estimation, pages 427-433, 1982.

S. Billings and K. Tsang. Spectral analysis for non-linear systems, part i: Parametric non-linear spectral analysis. Mechanical Systems and Signal Processing, 3(4):319-339, 1989a.

S. Billings and K. Tsang. Spectral analysis for non-linear systems, part ii: Interpretation of non-linear frequency response functions. Mechanical Systems and Signal Processing, 3 (4):341-359, 1989b.

S. Billings, M. Korenberg, and S. Chen. Identification of non-linear output-affine systems using an orthogonal least-squares algorithm. International Journal of Systems Science, 19(8):1559-1568, 1988.

S. Billings, S. Chen, and M. Korenberg. Identification of mimo non-linear systems using a forward-regression orthogonal estimator. International Journal of Control, 49(6):21572189, 1989.

S. Billings, K. Tsang, and G. Tomlinson. Spectral analysis for non-linear systems, part iii: Case study examples. Mechanical Systems and Signal Processing, 4(1):3-21, 1990.

S.A. Billings. Nonlinear system identification: NARMAX methods in the time, frequency, and spatio-temporal domains. John Wiley \& Sons, 2013.

S.A. Billings and D. Coca. Identification of narmax and related models. Control Systems, Robotics and Automation, VI, 2002.

S. Boyd and L.O. Chua. Fading memory and the problem of approximating nonlinear operators with volterra series. Circuits and Systems, IEEE Transactions on, 32(11):11501161, 1985. 
S. Chen and S. Billings. Representations of non-linear systems: the narmax model. International Journal of Control, 49(3):1013-1032, 1989.

S. Chen. Local regularization assisted orthogonal least squares regression. Neurocomputing, 69(4):559-585, 2006.

S. Chen, S.A. Billings, and W. Luo. Orthogonal least squares methods and their application to non-linear system identification. International Journal of control, 50(5):1873-1896, 1989.

O. Christensen. An Introduction to Frames and Riesz Bases. Birkhauser, Boston, 2003.

L.O. Chua and C.Y. Ng. Frequency domain analysis of nonlinear systems: general theory. IEE Journal on Electronic Circuits and Systems, 3(4):165-185, 1979a.

L.O. Chua and C. Ng. Frequency-domain analysis of nonlinear systems: formulation of transfer functions. Electronic Circuits and Systems, IEE Journal on, 3(6):257-269, 1979b.

P.S. Churchland and T.J. Sejnowski. The computational brain. The MIT press, 1992.

D. Coca, M. Balikhin, S. Billings, H. Alleyne, M. Dunlop, and H. Luhr. Time-domain identification of nonlinear processes in space plasma turbulence using multi-point measurements. In Cluster-II Workshop Multiscale/Multipoint Plasma Measurements, volume 449 , page $111,2000$.

D. Cochran and J.J. Clark. On the sampling and reconstruction of time warped band-limited signals. In Proc. IEEE Int. Conf. Acoust., Speech, and Signal Proc, 1990.

P. Dayan and L.F. Abbott. Theoretical neuroscience, volume 806. Cambridge, MA: MIT Press, 2001.

P. Dayan and L. Abbott. Theoretical neuroscience: computational and mathematical modeling of neural systems. Journal of Cognitive Neuroscience, 15(1):154-155, 2003.

H. Diaz and A.A. Desrochers. Modeling of nonlinear discrete-time systems from inputoutput data. Automatica, 24(5):629-641, 1988.

M.N. Do, D. Marchand-Maillet, and M. Vetterli. On the bandwidth of the plenoptic function. Image Processing, IEEE Transactions on, 21(2):708-717, 2012.

R.J. Duffin and A.C. Schaeffer. A class of nonharmonic fourier series. Transactions of the American Mathematical Society, pages 341-366, 1952. 
Y.C. Eldar and T. Werther. General framework for consistent sampling in hilbert spaces. International Journal of Wavelets Multiresolution and Information Processing, 3(3):347359, 2005.

Y.C. Eldar. Sampling without input constraints: Consistent reconstruction in arbitrary spaces. In Sampling, Wavelets and Tomography. Eds. Birkhauser, 2003.

H.G. Feichtinger and K. Gröchenig. Theory and practice of irregular sampling. Wavelets: mathematics and applications, 1994:305-363, 1994.

H.G. Feichtinger, K. Gr, T. Strohmer, et al. Efficient numerical methods in non-uniform sampling theory. Numerische Mathematik, 69(4):423-440, 1995.

D. Florescu and D. Coca. A novel reconstruction framework for time-encoded signals with integrate-and-fire neurons. Neural computation, 27(9):1872-1898, 2015.

U. Friederich, D. Coca, S. Billings, and M. Juusola. Nonlinear identification for modeling and analysis of adaptive neuronal systems. In Front. Syst. Neurosci. Conference Abstract: Computational and systems neuroscience, 2009a.

U. Friederich, D. Coca, S. Billings, and M. Juusola. Invariant contrast coding in photoreceptors. In Front Neuroscience Conference Abstract: Computational and Systems Neuroscience, 2010.

U. Friederich, D. Coca, S. Billings, and M. Juusola. Data modelling for analysis of adaptive changes in fly photoreceptors. In Neural Information Processing, pages 34-48. Springer, 2009b.

E.H. Fung, Y. Wong, H. Ho, and M.P. Mignolet. Modelling and prediction of machining errors using armax and narmax structures. Applied Mathematical Modelling, 27(8):611$627,2003$.

D. Gabor. Theory of communication. part 1: The analysis of information. Electrical Engineers-Part III: Radio and Communication Engineering, Journal of the Institution of, 93(26):429-441, 1946.

M.N. Geffen, B.M. Broome, G. Laurent, and M. Meister. Neural encoding of rapidly fluctuating odors. Neuron, 61(4):570-586, 2009.

D.A. George. Continuous nonlinear systems. Technical report, DTIC Document, 1959.

D. Gontier and M. Vetterli. Sampling based on timing: Time encoding machines on shiftinvariant subspaces. Applied and Computational Harmonic Analysis, 36(1):63-78, 2014. 
K. Gröchenig. Reconstruction algorithms in irregular sampling. Mathematics of Computation, 59(199):181-194, 1992.

K. Gröchenig. A discrete theory of irregular sampling. Linear Algebra and its applications, 193:129-150, 1993.

K. Gröchenig and H. Razafinjatovo. On landau's necessary density conditions for sampling and interpolation of band-limited functions. Journal of the London Mathematical Society, 54(3):557-565, 1996.

K. Gröchenig and H. Schwab. Fast local reconstruction methods for nonuniform sampling in shift-invariant spaces. SIAM Journal on Matrix Analysis and Applications, 24(4):899913, 2003.

Y. Gu, P. Lucas, and J.P. Rospars. Computational model of the insect pheromone transduction cascade. PLoS Comput Biol, 5(3):e1000321, 2009.

L. Guo and S. Billings. A modified orthogonal forward regression least-squares algorithm for system modelling from noisy regressors. International Journal of Control, 80(3): 340-348, 2007.

G.H. Hardy. A theorem concerning fourier transforms. Journal of the London Mathematical Society, s1-8(3):227-231, 1933.

A.L. Hodgkin and A.F. Huxley. A quantitative description of membrane current and its application to conduction and excitation in nerve. The Journal of physiology, 117(4): 500-544, 1952.

X. Hong, S. Chen, and C.J. Harris. A-optimality orthogonal forward regression algorithm using branch and bound. Neural Networks, IEEE Transactions on, 19(11):1961-1967, $2008 \mathrm{a}$.

X. Hong, R.J. Mitchell, S. Chen, C.J. Harris, K. Li, and G.W. Irwin. Model selection approaches for non-linear system identification: a review. International journal of systems science, 39(10):925-946, 2008b.

E.M. Izhikevich. Dynamical systems in neuroscience. MIT press, 2007.

S. Jaffard. A density criterion for frames of complex exponentials. Michigan Mathematical Journal, 38:339-348, 1991.

J.P. Jones. Simplified computation of the volterra frequency response functions of nonlinear systems. Mechanical systems and signal processing, 21(3):1452-1468, 2007. 
C. Káldy, A. Lazar, E. Simonyi, and L. Toth. Time encoded communications for human area network biomonitoring. Department of Elec. Eng., Columbia University, New York, BNET Tech. Rep, pages 2-07, 2007.

A.J. Kim, A.A. Lazar, and Y.B. Slutskiy. System identification of drosophila olfactory sensory neurons. Journal of computational neuroscience, 30(1):143-161, 2011.

M. Korenberg, S. Billings, Y. Liu, and P. Mcllroy. Orthogonal parameter estimation algorithm for non-linear stochastic systems. International Journal of Control, 48(1):193-210, 1988.

M.J. Korenberg and I.W. Hunter. The identification of nonlinear biological systems: Volterra kernel approaches. Annals of biomedical engineering, 24(2):250-268, 1996.

V.A. Kotelnikov. On the carrying capacity of the ether and wire in telecommunications. In Material for the First All-Union Conference on Questions of Communication, Izd. Red. Upr. Svyazi RKKA, Moscow, 1933.

H. Landau. Necessary density conditions for sampling and interpolation of certain entire functions. Acta Mathematica, 117(1):37-52, 1967.

Z.Q. Lang and S. Billings. Output frequency characteristics of nonlinear systems. International Journal of Control, 64(6):1049-1067, 1996.

L. Lapicque. Recherches quantitatives sur l'excitation électrique des nerfs traitée comme une polarisation. J. Physiol. Pathol. Gen, 9(1):620-635, 1907.

A. Lazar, A., T. Toth, L., and S.E. K. An overcomplete stitching algorithm for time decoding machines. IEEE Transactions on Circuits and Systems, 55(9), 2008.

A.A. Lazar. Time encoding with an integrate-and-fire neuron with a refractory period. Neurocomputing, 58:53-58, 2004.

A.A. Lazar. Multichannel time encoding with integrate-and-fire neurons. Neurocomputing, 65:401-407, 2005.

A.A. Lazar. Time encoding machines with multiplicative coupling, feedforward, and feedback. IEEE Transactions on Circuits and Systems-II Express Briefs, 53(8):672-676, 2006a.

A.A. Lazar. A simple model of spike processing. Neurocomputing, 69(10-12):1081-1085, $2006 b$

A.A. Lazar and E.A. Pnevmatikakis. Faithful representation of stimuli with a population of integrate-and-fire neurons. Neural Computation, 20(11):2715-2744, 2008a. 
A.A. Lazar and E.A. Pnevmatikakis. Consistent recovery of sensory stimuli encoded with mimo neural circuits. Computational Intelligence and Neuroscience, 2010:13, 2009.

A.A. Lazar and E.A. Pnevmatikakis. Video time encoding machines. IEEE Transactions on Neural Networks, 22(3):461-473, 2011.

A.A. Lazar and L.T. Tóth. Sensitivity analysis of time encoded bandlimited signals. In Acoustics, Speech, and Signal Processing, 2004. Proceedings. (ICASSP '04). IEEE International Conference on, volume 2, pages ii -901-4 vol.2, may 2004a.

A. Lazar and E. Pnevmatikakis. A video time encoding machine. In Image Processing (ICIP), 2008 15th IEEE International Conference on, pages 717 -720, oct. 2008b.

A.A. Lazar. Information representation with an ensemble of hodgkin-huxley neurons. Neurocomputing, 70(10):1764-1771, 2007.

A.A. Lazar. Population encoding with hodgkin-huxley neurons. Information Theory, IEEE Transactions on, 56(2):821-837, 2010.

A.A. Lazar and Y. Slutskiy. Multisensory encoding, decoding, and identification. In Advances in Neural Information Processing Systems, pages 3183-3191, 2013.

A.A. Lazar and Y.B. Slutskiy. Identifying dendritic processing. In J. Lafferty, C.K.I. Williams, J. Shawe-Taylor, R. Zemel, and A. Culotta, editors, Advances in Neural Information Processing Systems 23, pages 1261-1269, 2010. spotlight presentation.

A.A. Lazar and Y.B. Slutskiy. Channel identification machines. Journal of Computational Intelligence and Neuroscience, 2012:1-20, July 2012.

A.A. Lazar and Y.B. Slutskiy. Channel identification machines for multidimensional receptive fields. Frontiers in computational neuroscience, 8, 2014a.

A.A. Lazar and Y.B. Slutskiy. Functional identification of spike-processing neural circuits. Neural computation, 26(2):264-305, 2014b.

A.A. Lazar and Y.B. Slutskiy. Spiking neural circuits with dendritic stimulus processors. Journal of computational neuroscience, 38(1):1-24, 2014c.

A.A. Lazar and L.T. Tóth. Time encoding and perfect recovery of bandlimited signals. In ICASSP (6), pages 709-712, 2003.

A.A. Lazar and L.T. Tóth. Perfect recovery and sensitivity analysis of time encoded bandlimited signals. Circuits and Systems I: Regular Papers, IEEE Transactions on, 51(10): 2060-2073, 2004b. 
A.A. Lazar, E.A. Pnevmatikakis, and Y. Zhou. Encoding natural scenes with neural circuits with random thresholds. Vision Research, 50(22):2200 - 2212, 2010.

A.A. Lazar, Y.B. Slutskiy, and Y. Zhou. Massively parallel neural circuits for stereoscopic color vision: Encoding, decoding and identification. Neural Networks, 63:254-271, 2015.

Y.W. Lee and T.L. Chang. Application of narx neural networks in thermal dynamics identification of a pulsating heat pipe. Energy Conversion and Management, 50(4):1069-1078, 2009.

I. Leontaritis and S.A. Billings. Input-output parametric models for non-linear systems part i: deterministic non-linear systems. International journal of control, 41(2):303-328, $1985 \mathrm{a}$.

I. Leontaritis and S.A. Billings. Input-output parametric models for non-linear systems part ii: stochastic non-linear systems. International journal of control, 41(2):329-344, 1985b.

I. Leontaritis, S. Billings, and S.U.D. of Control Engineering;. Identification of non-linear systems using parameter estimation techniques. In Proceedings of IEEE Conference of Control and Applications, pages 183-190, 1981.

L. Li and S. Billings. Estimation of generalized frequency response functions for quadratically and cubically nonlinear systems. Journal of Sound and Vibration, 330(3):461-470, 2011.

D. Linkens and M. Khelfa. Control strategies for nonlinear dynamics of muscle relaxant anaesthesia. Computer methods and programs in biomedicine, 37(1):1-30, 1992.

C. Mead. Neuromorphic electronic systems. Proceedings of the IEEE, 78(10):1629-1636, 1990.

A.W. Naylor and G.R. Sell. Linear Operator Theory in Engineering and Science. SpringerVerlag, 1982.

S. Nirenberg and C. Pandarinath. Retinal prosthetic strategy with the capacity to restore normal vision. Proceedings of the National Academy of Sciences, 109(37):15012-15017, 2012.

R.K. Pearson. Nonlinear input/output modelling. Journal of Process Control, 5(4):197-211, 1995.

R.K. Pearson. Discrete-time dynamic models. Oxford University Press, 1999. 
J.W. Pillow and E.P. Simoncelli. Dimensionality reduction in neural models: an information-theoretic generalization of spike-triggered average and covariance analysis. Journal of vision, 6(4):9, 2006.

E. Pisoni, M. Farina, C. Carnevale, and L. Piroddi. Forecasting peak air pollution levels using narx models. Engineering Applications of Artificial Intelligence, 22(4):593-602, 2009.

E. Roza. Analog-to-digital conversion via duty-cycle modulation. Circuits and Systems II: Analog and Digital Signal Processing, IEEE Transactions on, 44(11):907 -914, nov 1997.

A.A.P. Santos, N.C.A. da Costa, and L. dos Santos Coelho. Computational intelligence approaches and linear models in case studies of forecasting exchange rates. Expert Systems with Applications, 33(4):816-823, 2007.

C. Shannon. Communication in the presence of noise. In Proceedings of the I.R.E., volume 37, pages 10-21, January 1949.

S.J. Slee, M.H. Higgs, A.L. Fairhall, and W.J. Spain. Two-dimensional time coding in the auditory brainstem. The Journal of Neuroscience, 25(43):9978-9988, 2005.

C. Smith. Biology of sensory systems. John Wiley \& Sons, 2008.

Z. Song, D. Coca, S. Billings, M. Postma, R. Hardie, and M. Juusola. Biophysical modelling of a drosophila photoreceptor. 2009.

Z. Song, M. Postma, S.A. Billings, D. Coca, R.C. Hardie, and M. Juusola. Stochastic, adaptive sampling of information by microvilli in fly photoreceptors. Current Biology, 22(15):1371-1380, 2012.

T. Strohmer. Irregular sampling, frames and pseudo-inverse. na, 1991.

M. Thomson, S. Schooling, and M. Soufian. The practical application of a nonlinear identification methodology. Control Engineering Practice, 4(3):295-306, 1996.

E.C. Titchmarsh. The zeros of certain integral functions. Proceedings of the London Mathematical Society, 2(1):283-302, 1926.

L.N. Trefethen and D. Bau III. Numerical linear algebra, volume 50. Siam, 1997.

H. Tuckwell. Introduction to theoretical neurobiology. Vol. 1, Linear cable theory and dendritic structure. Cambridge: Cambridge University Press, 1988. 
M. Unser. Splines: A perfect fit for signal and image processing. Signal Processing Magazine, IEEE, 16(6):22-38, 1999.

M. Unser. Sampling-50 years after shannon. Proceedings of the IEEE, 88(4):569-587, 2000

V. Volterra. Theory of functionals and of integral and integro-differential equations. Courier Corporation, 2005.

H.L. Wei, Y. Zheng, Y. Pan, D. Coca, L.M. Li, J.E. Mayhew, S. Billings, et al. Model estimation of cerebral hemodynamics between blood flow and volume changes: a databased modeling approach. Biomedical Engineering, IEEE Transactions on, 56(6):1606$1616,2009$.

M.C.K. Wu, S.V. David, and J.L. Gallant. Complete functional characterization of sensory neurons by system identification. Annu. Rev. Neurosci., 29:477-505, 2006.

R. Young. An Introduction to Nonharmonic Fourier Series. Academic Press, New York, 1980.

H. Zhang and S. Billings. Analysing non-linear systems in the frequency domain-i. the transfer function. Mechanical Systems and Signal Processing, 7(6):531-550, 1993.

D. Zhu, M. Balikhin, M. Gedalin, H. Alleyne, S. Billings, Y. Hobara, V. Krasnosel'Skikh, M. Dunlop, and M. Ruderman. Nonlinear dynamics of foreshock structures: Application of nonlinear autoregressive moving average with exogenous inputs model to cluster data. Journal of Geophysical Research: Space Physics (1978-2012), 113(A4), 2008. 